\title{
The Computation and Visualization of Uncertainty in Surgical Navigation
}

by

\author{
Amber Lea Simpson \\ A thesis submitted to the \\ School of Computing \\ in conformity with the requirements for \\ the degree of Doctor of Philosophy \\ Queen's University \\ Kingston, Ontario, Canada \\ January 2010
}

Copyright (c) Amber Lea Simpson, 2010 


\begin{abstract}
The subject of this dissertation is the calculation and visualization of intraoperative measurement uncertainty in computer-assisted surgical procedures. Error is the difference between the observed or measured value and the true value (called groundtruth) of a quantity. Uncertainty is the unknown difference between the measured and true values, and exists in the absence of knowledge of ground truth.

If one has an algorithm for computing the ground truth then one can get an accurate estimate of error. However, in computer-assisted surgery, the ground truth is often unknown. The introduction of error to surgical procedures is inevitable: it cannot be avoided by simply taking very careful measurements, providing more accurate algorithms, or by improving instrument calibration. One can only reduce errors as much as reasonably possible, calculate a reliable estimate of the uncertainty, and provide a meaningful way to convey this uncertainty information to clinicians.

In this dissertation, I demonstrate that the visualization of registration uncertainty improves surgical navigation and that real-time computation of intraoperative measurement uncertainty is possible. In an extensive user study of surgeons and surgical residents, I compare methods of visualizing intraoperative uncertainty and determine that there are several methods of effectively conveying uncertainty in surgical navigation.
\end{abstract}




\section{Acknowledgments}

I would like to thank my supervisors Randy Ellis and James Stewart for recognizing my research potential before it was visible to me and giving me the opportunity to do research that I truly love.

Computer-aided surgery requires the juxtaposition of many specialized minds. Many thanks to everyone in the Medical Computing Laboratory and Human Mobility Research Centre, in particular Elvis Chen, Siyang Chen, Steve Devlin, Heather Grant, Manuela Kunz, Burton Ma, Leone Ploeg, Paul St. John, and Thomas Tang. Special mention to my dad, for helping me to sand hundreds of bone models. I extend thanks to my clinical collaborators Dr. Dan Borschneck and Dr. Ted Vasarhelyi. To all of the surgeons, surgical residents, and graduate students that took part in my user studies, no amount of chocolate and Tim Horton's gift certificates can say thank you enough.

My deepest gratitude goes to my friends especially, Michelle Crane, Jiro Inoue, Ben Hall, Lyndsay Puterbough, Ingrid Wang, Sarah Whittaker, and Kitty Wong.

Finally, Mom and Dad, this one is for us! 


\section{Statement of Originality}

I certify that this dissertation is my original work and all necessary references have been cited. 


\section{Contents}

Abstract $\quad$ i

Acknowledgments $\quad$ ii

Statement of Originality $\quad$ iii

Contents $\quad$ iv

List of Tables viii

List of Figures $\quad x$

Glossary $\quad$ xiv

Chapter 1: Introduction $\quad 1$

1.1 Stages of Computer-Aided Orthopaedic Surgery . . . . . . . . . . . . 1

1.2 Uncertainty . . . . . . . . . . . . . . . . . . 3

1.3 Uncertainty Sources . . . . . . . . . . . . . . . . . . 5

1.4 Contributions . . . . . . . . . . . . . . . . . . . . 9

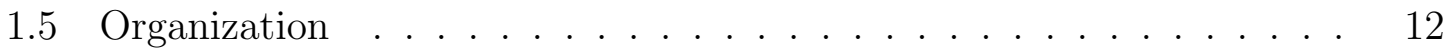

Chapter 2: $\quad$ Review of Relevant Literature 13

2.1 An Overview of Major Navigation Paradigms in CAS . . . . . . . . . 13

2.1.1 Navigation Using Preoperative Images . . . . . . . . . . . . . 14

2.1.2 Direct Navigation . . . . . . . . . . . . . . . . . . . . . . . 15

2.1.3 Image-free Navigation . . . . . . . . . . . . . . . . . . 18

2.1.4 No Navigation . . . . . . . . . . . . . . . . . . . . . 20

2.2 A Classification of Error and Uncertainty Analysis in CAS . . . . . . 22

2.2.1 Registration Error . . . . . . . . . . . . . . 23

2.2.2 Registration Uncertainty . . . . . . . . . . . . . 26

2.2 .3 Tracking Error . . . . . . . . . . . . . . . . . . . . . . . . . 29

2.2.4 Tracking Uncertainty . . . . . . . . . . . . . . . . . 30 
2.3 An Overview of Visualization in CAS . . . . . . . . . . . . . 31

2.3.1 Navigation Using Preoperative Images . . . . . . . . . . . . 31

2.3 .2 Direct Navigation . . . . . . . . . . . . . . . . . . . . . 32

2.3 .3 Image-free Navigation . . . . . . . . . . . . . . . . . . 36

2.4 Related Work in Uncertainty Visualization . . . . . . . . . . . . 36

2.4.1 Perceptual and Cognitive Theory for Visualization Design . . 36

2.4.2 Uncertainty Visualization Methods . . . . . . . . . . . . 42

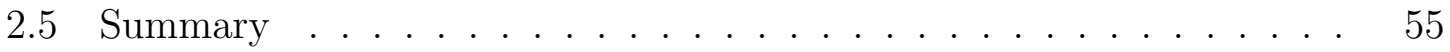

Chapter 3: Study: Does Registration Uncertainty Improve Surgical Navigation? $\quad 58$

3.1 Registration Error . . . . . . . . . . . . . . . . . . . . 59

3.1.1 Registration Uncertainty . . . . . . . . . . . . . . . . . 59

3.2 Osteoid Osteoma Excision . . . . . . . . . . . . . . . . . . . . . 61

3.3 Methodology . . . . . . . . . . . . . . . . . . . . . . . 61

3.3.1 Registration Uncertainty Source . . . . . . . . . . . . . . . . 61

3.3 .2 Visualization Method . . . . . . . . . . . . . . . . . . . . 62

3.3 .3 User Study . . . . . . . . . . . . . . . . . . . . . . . . . . 64

3.3 .4 Subjects . . . . . . . . . . . . . . . . . 66

3.3 .5 Apparatus . . . . . . . . . . . . . . . . . . 66

3.3 .6 Stimuli . . . . . . . . . . . . . . . . . . . 67

3.3 .7 Design . . . . . . . . . . . . . . . . . . . . . . 68

3.4 Results . . . . . . . . . . . . . . . . . . . 70

3.4.1 Hypothesis 1: Fewer attempts with uncertainty . . . . . . . 71

3.4.2 Hypothesis 2: Fewer incomplete tasks with uncertainty . . . . 72

3.4.3 Hypothesis 3: Greater fraction of the target probability distribution is covered . . . . . . . . . . . . 73

3.4.4 Hypothesis 4: Attempts match the actual uncertainty distribution 75

3.5 Discussion . . . . . . . . . . . . . . . . . . . . 77

$\begin{array}{lll}\text { Chapter 4: } & \text { Computing Intraoperative Uncertainty } & \mathbf{7 9}\end{array}$

4.1 UKF Target Tracking Algorithm I . . . . . . . . . . . . . . . . 81

4.1 .1 Experiments . . . . . . . . . . . . . . . . . . . . . 84

4.1 .2 Validation . . . . . . . . . . . . . . . . . 86

4.1 .3 Results . . . . . . . . . . . . . . . . . . . . 86

4.1 .4 Discussion . . . . . . . . . . . . . . . . . . . . . . . 87

4.2 UKF Tracking Algorithm II . . . . . . . . . . . . . . . . . . . 91

4.2 .1 Experiments . . . . . . . . . . . . . . . . . . . . . . . 93

4.2 .2 Validation . . . . . . . . . . . . . . . . . . . 94

4.2 .3 Results . . . . . . . . . . . . . . . . . . . . . 94 
4.2.4 Discussion ...................... . . . . 97

4.3 UKF Calibration Algorithm . . . . . . . . . . . . . . . . . 97

4.3.1 Experiments . . . . . . . . . . . . . . . . 98

4.3 .2 Results . . . . . . . . . . . . . . . . . . . . 99

4.3 .3 Discussion . . . . . . . . . . . . . . . . . 103

4.4 A Combined Estimate of Tracking and Calibration Uncertainty . . . 105

4.4.1 Experiments . . . . . . . . . . . . . . 107

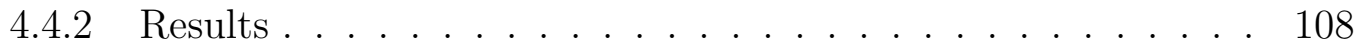

4.5 Discussion . . . . . . . . . . . . . . . . . . . . . . . 108

Chapter 5: Study: How Do Guidance and Visualization Affect a

Surgeon's Performance? 113

5.1 Pedicle Screw Fixation . . . . . . . . . . . . . . . . . . . . . . . 114

5.2 Methodology .......................... 119

5.2.1 Uncertainty Sources . . . . . . . . . . . . . . . . 119

5.2 .2 Navigation Methods . . . . . . . . . . . . . . . . . 123

5.2.3 Uncertainty Visualization Methods . . . . . . . . . . . . . . . 125

5.2 .4 Subjects . . . . . . . . . . . . . . . . . . 131

5.2.5 Apparatus ......................... 132

5.2 .6 Conditions . . . . . . . . . . . . . . . . . . . . 133

5.2.7 Hypotheses . . . . . . . . . . . . . . . . . . 135

5.2 .8 Design . . . . . . . . . . . . . . . . . . 136

5.3 Results . . . . . . . . . . . . . . . . . . . 143

5.3.1 Experiment 1 . . . . . . . . . . . . . . . . 143

5.3 .2 Experiment 2 . . . . . . . . . . . . . . . . 147

5.3.3 Exit Questions . . . . . . . . . . . . . . 157

5.4 Discussion . . . . . . . . . . . . . . . . . . 158

$\begin{array}{ll}\text { Chapter 6: } & 167\end{array}$

6.1 Contributions . . . . . . . . . . . . . . . 167

6.1.1 Registration Uncertainty Improves Surgical Navigation . . . . 167

6.1.2 Real-time Computation of Intraoperative Measurement Uncertainty is Possible . . . . . . . . . . . . 168

6.1.3 Uncertainty Was Studied in an Extensive Trial of Surgeons Stratified by Experience . . . . . . . . . . . . . 168

6.1.4 There is More Than One Way to Effectively Convey Uncertainty in Surgical Navigation . . . . . . . . . . . . . . . . 169

6.1.5 Criteria Have Been Established for Computing and Visualizing Uncertainty in Surgical Navigation . . . . . . . . . . . . 169

6.2 Future Work . . . . . . . . . . . . . . . . . . 170 
6.3 Conclusions . . . . . . . . . . . . . . . . . . . . . . . . . 171

$\begin{array}{ll}\text { Bibliography } & 172\end{array}$

Appendix A: Kalman Filtering $\quad 190$

A.1 State Estimation . . . . . . . . . . . . . . . . . . . 190

A.2 Parameter Estimation . . . . . . . . . . . . . . . . . 191

A.3 Dual Estimation . . . . . . . . . . . . . . . . . . . . . . . 191

A.4 The Discrete Kalman Filter . . . . . . . . . . . . . . . . . . . . . 192

A.5 The Unscented Kalman Filter . . . . . . . . . . . . . . . . . . . . . . 192

$\begin{array}{ll}\text { Appendix B: Bootstrap Method } & 196\end{array}$

$\begin{array}{ll}\text { Appendix C: Consent Form } & 197\end{array}$

Appendix D: A Note On Constructing Plastic Bone Models 200

$\begin{array}{ll}\text { Appendix E: Consent Form } & 204\end{array}$

$\begin{array}{ll}\text { Appendix F: Questionnaire } & 206\end{array}$ 


\section{List of Tables}

3.1 Cumulative Fraction of Uncertainty Distribution Covered After Each

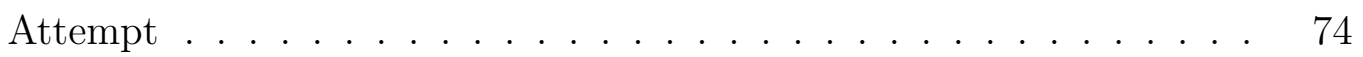

3.2 Box's M Test Results for Equality of Covariances for Each Subject . . 76

3.3 Comparison of Ratios of Standard Deviations in Principal Directions $\quad 77$

5.1 A sample schedule of tasks for one subject for Experiment 1. . . . . . 138

5.2 A sample schedule of tasks for one subject for Experiment 2 for all five conditions. Each entry is listed as bone, pedicle, and uncertainty registration instance label. . . . . . . . . . . . . . . . . . . . . . . . . 140

5.3 Intra-rater Reliability of Measurements in Experiment 1 . . . . . . . 145

5.4 Reported Means For All Measurements According Visualization Type 149

5.5 Reported Means For All Measurements According to Experience Level and Visualization Type . . . . . . . . . . . . . . . . . 155

5.6 Subject Responses to Exit Questions . . . . . . . . . . . . . . . . . . 159

5.7 Subject Responses: How Uncertainty Methods Were Used For Guidance160

5.8 Subject Responses: How Navigation Methods Were Used For Guidance 160

5.9 Summary of Experiment Results for Accepted Hypotheses . . . . . . 161

5.10 Summary of Experiment Results for Hypotheses Accepted for Equiva-

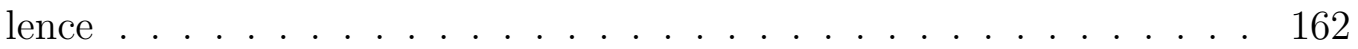


5.11 Summary of Experiment Results for Rejected Hypotheses . . . . . . . 163 


\section{List of Figures}

1.1 The plan-scan-operate paradigm of CAOS. . . . . . . . . . . . 2

1.2 Three stages of CAOS. . . . . . . . . . . . . . . 4

1.3 Distortion in CT. . . . . . . . . . . . . . . . . . . . 7

1.4 Intraoperative propagation of covariance. . . . . . . . . . . . . . 10

2.1 A guidance system that uses preoperative images. . . . . . . . . . . 16

2.2 Navigation system used by Kingston General Hospital. . . . . . . . . 17

2.3 Intraoperative data collection in an image-free navigation system. . . 18

2.4 A screen capture of an image-free navigation system. . . . . . . . . . 19

2.5 Creation of the patient-specific drill template. . . . . . . . . . . . 20

2.6 Intraoperative positioning of patient-specific template. . . . . . . . . 21

2.7 The Taylor frame before and after correction. . . . . . . . . . . 22

2.8 CT scan of Taylor frame rings. . . . . . . . . . . . . . . 23

2.9 A screen capture of the three views of preoperative planning. . . . . . 32

2.10 Cadaver feet reconstructed from CT using Advantage Workstation. . 34

2.11 Comparison of isosurface extraction and segmented model. . . . . . . 35

2.12 A screen capture of the Ci Knee Essential navigation system. . . . . . 37

2.13 Examples of uncertainty glyphs. . . . . . . . . . . . . . 43

2.14 Uncertainty cones in the corpus callosum. . . . . . . . . . . . . 44 
2.15 Uncertainty representation in EEG. . . . . . . . . . . . . . . 44

2.16 Uncertainty in the pose of a coordinate system. . . . . . . . . 45

2.17 Uncertainty visualization by surface displacement. . . . . . . . . . 46

2.18 A probabilistic surface. . . . . . . . . . . . . . . 47

2.19 Visualization of satellite image data. . . . . . . . . . . . 47

2.20 Uncertainty representation by color, aliasing, and transparency . . . . 48

2.21 Three textures representing uncertainty in flow fields. . . . . . . . . 49

2.22 Hue, texture, and color representation of uncertainty. . . . . . . 49

2.23 Two frames extracted from an uncertainty animation. . . . . . . 50

2.24 An animated uncertainty glyph. . . . . . . . . . . . 50

2.25 Confidence intervals for weather forecasting. . . . . . . . . . . 52

2.26 Representation of uncertainty in tomor typing. . . . . . . . . . 53

2.27 Direct volume rendering of brain image data. . . . . . . . . 54

2.28 Common chart types with error bars. . . . . . . . . . 55

2.29 Graph representations of radiation dosing uncertainty. . . . . . . . 56

3.1 Estimates distributions of registration parameters. . . . . . . . 60

3.2 Model of the proximal femur used in experiments. . . . . . . . . . 63

3.3 A rendering of possible tool trajectories. . . . . . . . . 63

3.4 Illustration of the path distribution volume. . . . . . . . . . . 64

3.5 The volume rendered path distribution. . . . . . . . . . . . . 65

3.6 Path distribution rendered as a volume. . . . . . . . . . . 65

3.7 Uncertainty superimposed on a model of the proximal femur. . . . . 65

3.8 Illustration of apparatus used study. . . . . . . . . . . 67

3.9 User interfaces used in the study. . . . . . . . . . . . . . . 69 
3.10 Difference in the number of attempts with and without uncertainty. . 71

3.11 An example of subject's probes. . . . . . . . . . . . . . . . 72

3.12 An example of subject's probes. . . . . . . . . . . . . 73

3.13 Distribution of displacements of points touched. . . . . . . . . . 75

4.1 Target model and tracking system configuration. . . . . . . . . 85

4.2 Difference in pose computed by UKF and the simulated result. . . . . 87

4.3 Simulation results of the UKF method. . . . . . . . . . . . . . . 88

4.4 Simulation results of our method using missing data. . . . . . . . . . 89

4.5 Difference in pose computed by the UKF and simulated result. . . . . 95

4.6 Comparison of covariance of UKF and HEIV estimates. . . . . . . . . 96

4.7 Difference in calibration result for UKF and least squares. . . . . . . 100

4.8 Difference in covariance result for UKF and least squares. . . . . . . . 101

4.9 Calibration covariance estimates compared to bootstrapped covariance. 104

4.10 Calibration covariance estimates compared to bootstrapped covariance. 105

4.11 Calibration covariance estimates compared to bootstrapped covariance. 106

4.12 Dual calibration and tracking covariance estimates comparison. . . . . 109

4.13 Dual calibration and tracking covariance estimates comparison. . . . . 110

4.14 Dual calibration and tracking covariance estimates comparison. . . . . 111

5.1 Vertebral anatomical terms. . . . . . . . . . . . . . . 115

5.2 Three views illustrating good pedicle screw placement. . . . . . . . 115

5.3 Pedicle screws from the Expedium Spine System. . . . . . . . . . . 116

5.4 The VectorVision spine navigation system in use clinically. . . . . . . 118

5.5 FLE computed at $10^{\circ}$ increments from $0^{\circ}$ (top left) to $60^{\circ}$ (bottom right). 120

5.6 The three orthogonal view interaction method used in the study. . . . 124 
5.7 The multiple coronal slice interaction method used in the study. . . . 126

5.8 Ellipse and cone uncertainty visualization method. . . . . . . . . . 128

5.9 Multi-line visualization method with orthogonal view navigation. . . . 129

5.10 Multi-line visualization method using multiple coronal slice navigation. 130

5.11 Select Expedium Spine System tools. . . . . . . . . . . . . . . 133

5.12 Experiment setup used in the study of pedicle screw insertions. . . . . 134

5.13 Examples of breaching of the pedicle. . . . . . . . . . . . . . 141

5.14 Rotation error measures used in the study. . . . . . . . . . . . . . . . 142

5.15 Mean ranking score for each subject by visualization method. . . . . . 146

5.16 Mean score for each experience level by visualization. . . . . . . . . . 147

5.17 Rotation error, impingement, and time plotted against visualization. . 150

5.18 Mean rotation error, impingement, and time plotted against experience. 156

A.1 Unscented Kalman Filter equations. . . . . . . . . . . . . . . . . 195

D.1 The two-part mould with the template. . . . . . . . . . . . . 202

D.2 The original prototyped model and the final cast model. . . . . . . . 203 


\section{Glossary}

anisotropic noise A noise model in which the noise differs in the $x, y$, and $z$ directions [74].

ANOVA Analysis of variance (ANOVA) describes a group of inferential statistical procedure that evaluates whether or not there is a difference between at least two means in a set of data for which two or means can be computed [95].

axial Anatomical plane that divides the body into upper and lower halves. Also called the transverse or horizontal plane [117].

bias Systematic error introduced into sampling or testing by selecting or encouraging one outcome or answer over others [71].

confidence interval An interval in which a measurement falls corresponding to a given probability, usually symmetric around the mean [113].

coronal Plane that divides the body horizontally from front to back [117].

covariance A measure of the strength of correlation between two or more sets of random variables [113]. 
extended Kalman filter The extended Kalman filter (EKF) is a Kalman filter that can be used for nonlinear estimation if the process and observation models can be linearized using a Taylor series expansion [53].

Gaussian The probability density function of a Gaussian noise amplitude takes on the shape of a normal bell-shaped curve. It can be shown mathematically that when a number of independent random variables are added together, the summed effect can be described closely by a Gaussian probability density, regardless of the shape of the independent densities [67].

heteroscedastic noise A noise model in which the noise is anisotropic and inhomogeneous [74].

IREDs Infrared emitting diodes (IREDs) are spherical, retro-reflective markers that reflect infrared light, emitted by illuminators on the optical tracking system [80].

isotropic noise A noise model in which noise is equal in all directions [74].

Kalman filtering A nonlinear estimation method that estimates the mean and covariance of the true system state from noisy measurements [39].

Kruskal-Wallis A nonparametric test employed with ordinal data for comparing two or more independent samples [95].

leptokurtic A distribution with a high peak [113].

Mann-Whitney A nonparametric test employed with ordinal data for comparing two independent samples [95]. 
mean The average score in a distribution [95].

mutual information Mutual information measures the statistical similarity between two random variables by comparing the amount of information that the first random variable shares with the second [63].

noise Errors arising from random effects [103].

ordinal A measurement level where numbers represent rank-orders such that the differences between adjacent values are not important. For example, restaurant ratings are ordinal measurements [95].

percutaneous Performed through the skin [71].

pose Position and orientation of a rigid target in space.

probability density function The probability density function of a continuous distribution is the derivative of the distribution function. The distribution function describes the probability that a variable takes a value less than or equal to a given number [113].

sagittal Plane that divides the body vertically into right and left halves [117].

significant Indicates level of significance. For example, the notation $p<.01$ indicates statistical significance, that there is less than a $1 \%$ likelihood that the difference is due to chance alone [95].

standard deviation A measure of the variability in a population. The standard deviation is the square root of the variance [95]. 
unscented Kalman filter A nonlinear estimation method based on the idea that it is easier to approximate a Gaussian distribution than to approximate an arbitrary nonlinear function [39].

variance A measure of the variability in a population. Variance is the square of the standard deviation [95].

white noise Noise value is not correlated in time [67]. 


\section{Chapter 1}

\section{Introduction}

Medicine is a science of uncertainty and an art of probability. Sir William Osler, 1849-1919, Canadian physician and educator.

Computer-assisted surgery (CAS) combines the accuracy and repeatability of machines with the judgement and problem-solving skills of humans to produce surgical results that neither could achieve independently. CAS has improved the practice of surgery in two ways. First, more accurate and precise tools reduce complications and

improve patient outcomes. Second, surgical procedures impossible with traditional surgical technique mean that patients not previously eligible for surgical treatment are treated.

\subsection{Stages of Computer-Aided Orthopaedic Surgery}

Computer-assisted orthopaedic surgery (CAOS) is the application of CAS to the surgical subspecialty of orthopaedics. The particular CAOS paradigm that I will address involves the scan-plan-operate paradigm illustrated in Figure 1.1. Preoperatively, a 

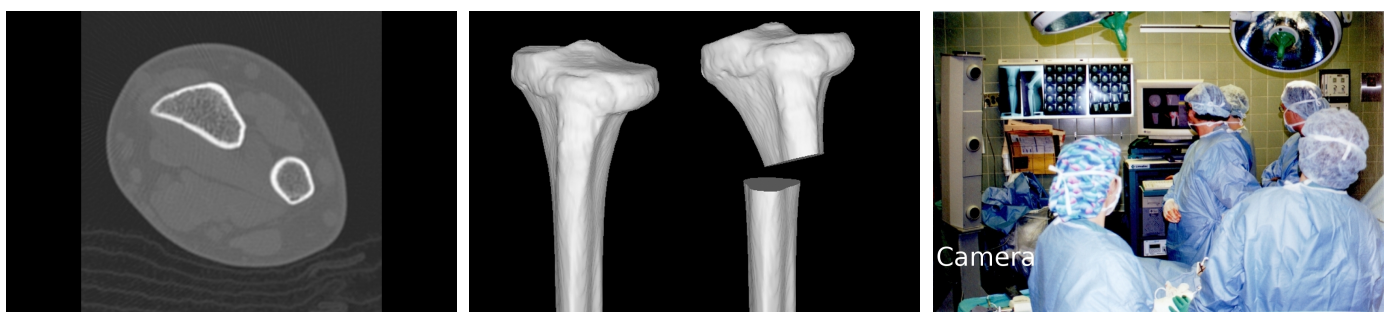

Figure 1.1: The scan-plan-operate paradigm of CAOS uses (left) a CT scan of the anatomy to produce (center) a patient-specific plan executed (right) in surgery. Note the presence of the camera and location of the computer in the room.

CT scan of the patient anatomy is obtained. A patient-specific 3D model is constructed from the CT scan. The surgeon uses this model to create a preoperative plan in which the required cuts to the bone are defined. The plan is executed by the surgeon in the operating room.

In the operating room, there are three major technical tasks that must be accomplished. Each task consists of the computation of a transformation ${ }^{1}$.

1. Tracking. Infrared emitting diodes (IREDs) are attached to rigid targets with a known geometry. These targets are attached to the surgical tool and to the patient. A camera system located in the operating theater measures the three dimensional position of the IREDs and, thus, the position and orientation of the tool or patient. Each target is defined in a common coordinate frame; hence, the transformation between any two targets can be determined.

2. Calibration. Calibration establishes the relationship between the model of the surgical tool and the target affixed to the tool. Calibration defines the transformation between the model of the tool and target.

\footnotetext{
${ }^{1} \mathrm{~A}$ transformation is a linear map from one coordinate frame to another.
} 
3. Registration. Registration establishes the relationship between the patient's real anatomy in the coordinate frame of the camera. A set of registration points is collected from the surface of the patient's bone using a tracked and calibrated surgical tool. These points are matched to the surface of the computer model to give the transformation between the model and the patient.

\subsection{Uncertainty}

The subject of this dissertation is the calculation and visualization of uncertainty in surgical procedures. Error is the difference between the observed or measured value and the true value (called ground-truth) of a quantity. If an algorithm exists for computing the ground truth then we can get an accurate estimate of error. However, in CAOS, the knowledge of ground truth is often unknown. Uncertainty is the unknown difference between the measured and true values, and exists in the absence of ground truth [103].

The National Institute of Standards and Technology (NIST) states unequivocally that "a measurement result is complete only when accompanied by a quantitative statement of its uncertainty" (in [103], pg. 11). Good science requires effectively communicating the reliability of results.

The definition of uncertainty can encompass a broad range of issues like inconsistency, doubtfulness, reliability, or inaccuracy [93]. I draw my definition from the guidelines for evaluating and expressing uncertainty in measurement results set by the NIST [103]. NIST classifies measurement uncertainty into two types. Type A methods are evaluated using any valid statistical method such as calculating the standard deviation of the mean of a series of independent samples or using analysis of 

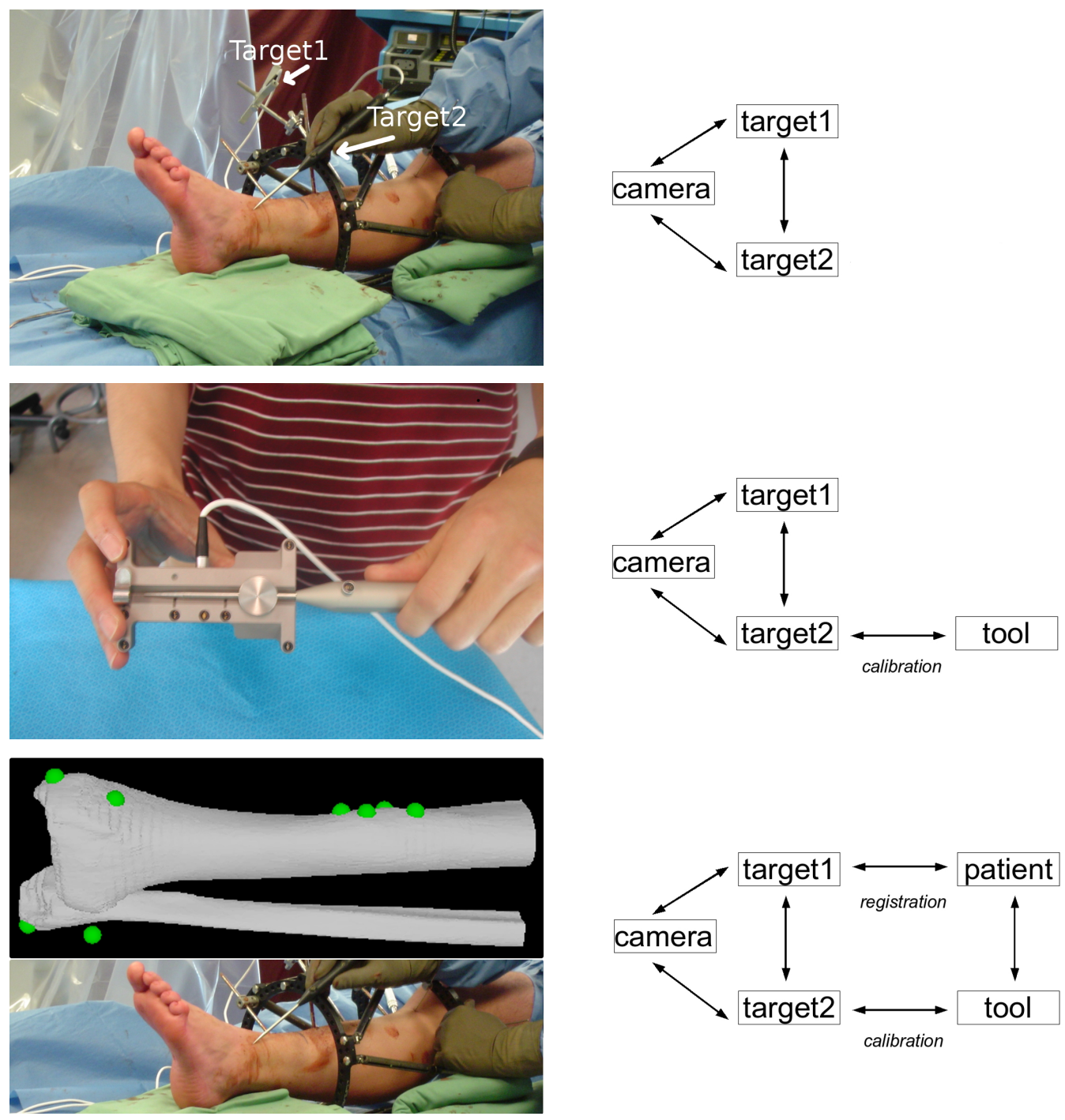

Figure 1.2: The three main tasks in CAOS. (Top) A camera located in the operating room tracks the positions and orientations of targets attached to the patient (target1) and surgical tool (target2). Tracking transforms the targets into the common camera coordinate frame. (Middle) Calibration establishes the spatial relationship between the camera coordinate frame and the coordinate frame of the surgical tool. (Bottom) Registration establishes the transformation of the patient model to the target affixed to the patient. Once registration and calibration are complete, the surgical tool and patient model are in a common coordinate frame. 
variance (ANOVA) to quantify random effects in particular measurements. Type B methods are evaluated based on scientific judgement, which may include previously measured data, general knowledge of materials and instruments, and manufacturer's specifications.

The NIST suggests an alternative nomenclature in which components of uncertainty are classified as those "arising from random effect" (called noise) or those "arising from systemic effect" (called bias). However, they acknowledge that this scheme is problematic because random components may become systemic [103].

Foody and Atkinson (in [27], pg. 26) offer a succinct definition: uncertainty is a "quantitative statement about the probability of error".

\subsection{Uncertainty Sources}

Errors and uncertainty are introduced in surgery when data is acquired (through an imaging modality or tracking system), transformed (by registration or segmentation), and displayed. The introduction of error to surgical procedures is inevitable. It cannot be avoided by simply taking very careful measurements, providing more accurate algorithms, or by improving instrument calibration. We can only reduce errors as much as reasonably possible, calculate a reliable estimate of the uncertainty, and provide a meaningful way to convey this uncertainty information to clinicians.

Possible sources of uncertainty in computer-assisted surgery are:

- Uncertainty is introduced any time data is acquired from a measurement device. For example, an optical tracking systems has limited accuracy so its measurements contain noise. Hence, the position and orientation of tracked targets may be uncertain. Similarly, measurements from image acquisition devices contain 
noise.

- Numerical methods used for tracking, calibration, and registration use approximations that introduce bias.

- Imaging devices can introduce bias during image acquisition. Figure 1.3 is a CT and fluoroscopy image that have been distorted by the presence of metallic objects.

- Visualization of 3D patient models requires interpolation in sampled data and the computation of transfer functions which introduce bias.

- Segmentation of anatomy requires determining the boundary between neighboring structures which introduces bias and noise.

Let us consider specific examples of covariances in surgical guidance. In an optical tracking system, two or three sensors compute the pose (position and orientation) of rigid targets from the raw positions of infrared emitting diodes. The uncertainty of the target pose can be represented as $C(x, y, z, \phi, \theta, \psi)$ where $x, y, z$ represent position and $\phi, \theta, \psi$ represent orientation in Euler angles about the $x, y, z$ axes.

Suppose that the surgical tool is calibrated by fixing the tip of the tool and rotating the tool about the tip. The measured data (consisting of target positions) are fit to a sphere which yields the center and radius of the sphere. The calibration uncertainty is represented as the covariance of the tip of the calibration tool $C\left(r, c_{x}, c_{y}, c_{z}\right)$ where $r$ and $c_{x}, c_{y}, c_{z}$ are the radius and center of the sphere.

Similarly, rigid registration uncertainty can be expressed as the covariance of registration parameters $C\left(t_{x}, t_{y}, t_{z}, r_{\phi}, r_{\theta}, r_{\psi}\right)$ where $t_{x}, t_{y}, t_{z}$ are the translational components and $r_{\phi}, r_{\theta}, r_{\psi}$ are rotational components expressed as Euler angles. 

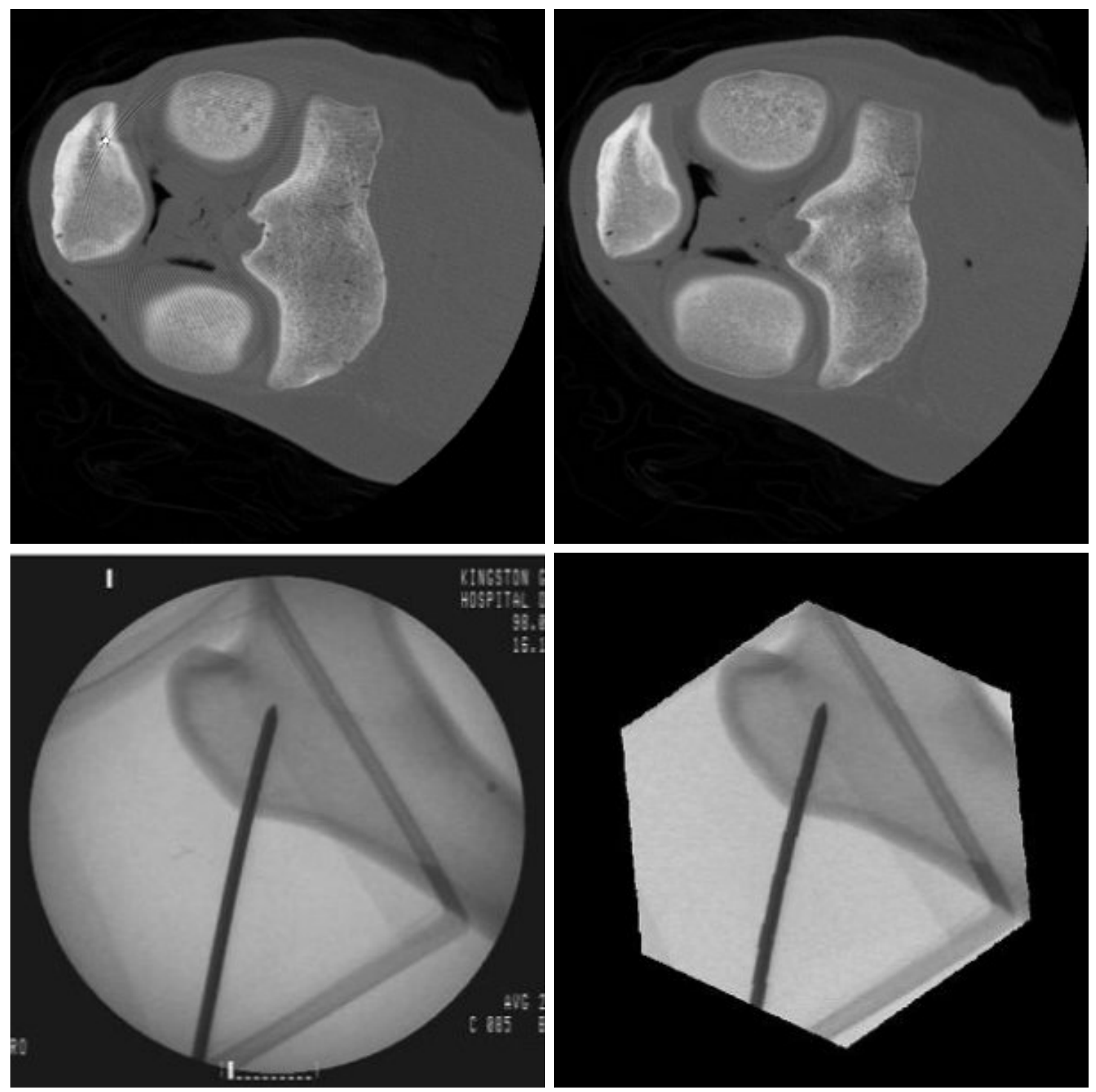

Figure 1.3: (Top) Distortion in a CT slice due to a metallic bead shown before (left) and (right) after correction. (Bottom) Distortion in a fluoroscopic image due to the presence of an external, unimaged metallic object (left) before and (right) after correction. 
Figure 1.4 demonstrates the relationship between tracking, registration, and calibration covariance estimates (uncertainty) in a computer guided surgical procedure and how these covariances propagate through other measurements. Assume that there is a target affixed to each of a pointing device, the patient, and a surgical tool. The first, second, and third columns of Figure 1.4 correspond to the pointer, patient, and surgical tool targets, respectively. In the first column, raw IRED positions $\left(1_{x y z}, \ldots, 4_{x y z}\right)$ of the pointer target are reported by the tracking system with corresponding covariance $C_{I}^{P O I N T}\left(1_{x y z}, \ldots, 4_{x y z}\right)$. A tracking algorithm takes as input these raw IRED positions (in camera coordinates) and outputs the pose of the pointer target $(x, y, z, \phi, \theta, \psi)$ (also in camera coordinates) with the associated covariance $C_{P}^{P O I N T}(x, y, z, \phi, \theta, \psi)$. A calibration method takes the pose in camera coordinates and computes the tip of the pointer $\left(r, c_{x}, c_{y}, c_{z}\right)$ (in the target's coordinate frame) with covariance $C_{T}^{P O I N T}\left(r, c_{x}, c_{y}, c_{z}\right)$. In the second column, raw IRED positions of the patient target are used to compute the pose of the patient target $(x, y, z, \phi, \theta, \psi)^{2}$ in camera coordinates with covariance $C_{P}^{P A T}(x, y, z, \phi, \theta, \psi)$. The pointer tip $\left(r, c_{x}, c_{y}, c_{z}\right)$ with covariance $C_{T}^{P O I N T}\left(r, c_{x}, c_{y}, c_{z}\right)$ and the pose of the patient target $(x, y, z, \phi, \theta, \psi)$ with the associated covariance $C_{P}^{P A T}(x, y, z, \phi, \theta, \psi)$ are input to the registration which outputs the transformation from the patient target coordinate frame with associated transformation $\left(t_{x}, t_{y}, t_{z}, r_{\phi}, r_{\theta}, r_{\psi}\right)$ with covariance $C_{R}^{C}\left(t_{x}, t_{y}, t_{z}, r_{\phi}, r_{\theta}, r_{\psi}\right)$ to $\mathrm{CT}$ coordinates. In the third column, the covariance in the tip of the surgical tool $C_{S}^{T}\left(r, c_{x}, c_{y}, c_{z}\right)$ is propagated in the same fashion as the pointer covariance $C_{T}^{P O I N T}\left(r, c_{x}, c_{y}, c_{z}\right)$. Finally, the visualization step propagates the covariance of the surgical tool $C_{S}^{T}\left(r, c_{x}, c_{y}, c_{z}\right)$ and the covariance of the registration

\footnotetext{
${ }^{2}$ For the sake of simplicity, the parameters that describe IRED positions $\left(1_{x y z}, \ldots, 4_{x y z}\right)$, target pose $(x, y, z, \phi, \theta, \psi)$, and tip $\left(r, c_{x}, c_{y}, c_{z}\right)$ have the same notation in each column even though the pointer, patient, and tool targets have distinct values.
} 
step $C_{R}^{C}\left(t_{x}, t_{y}, t_{z}, r_{\phi}, r_{\theta}, r_{\psi}\right)$ to the pose of the tool in CT coordinates with associated covariance $C_{S}^{C}(x, y, z, \phi, \theta, \psi)$.

Currently, computer-assisted surgery largely ignores the computation and visualization of uncertainty in data. Uncertainty might be ignored because of the inherent difficulty in expressing and computing uncertainty during surgery. It might also be that there is a lack of meaningful methods of visualizing uncertainty and data.

The visualization of uncertainty is absent from current commercial computerassisted surgery systems. In fact, the only error information (if any) given to surgeons is a scalar value representing registration error. Anatomical data is presented to the surgeon as though it were perfect. A surgeon who is not aware of this uncertainty can make critical errors (consider, for example, inserting screws into the spine). This work addresses the existence of uncertainty in surgery and offers a first step toward helping surgeons make informed decisions in the presence of imperfect data.

\subsection{Contributions}

There are three research questions central to this dissertation. The first question is

- Does uncertainty visualization improve the accuracy of surgical navigation?

Commercial surgery systems offer little error information to surgeons. For example, the VectorVision system (BrainLAB Inc., Westchester, Illinois, USA) indicates registration error during navigation as a single scalar value. My colleagues and I performed a user-study that demonstrated that if registration uncertainty was shown, subjects were able to localize a blind tumor with fewer attempts and failures than when uncertainty was not shown. We demonstrated that subjects used the uncertainty visualization to guide their search for the tumor. 


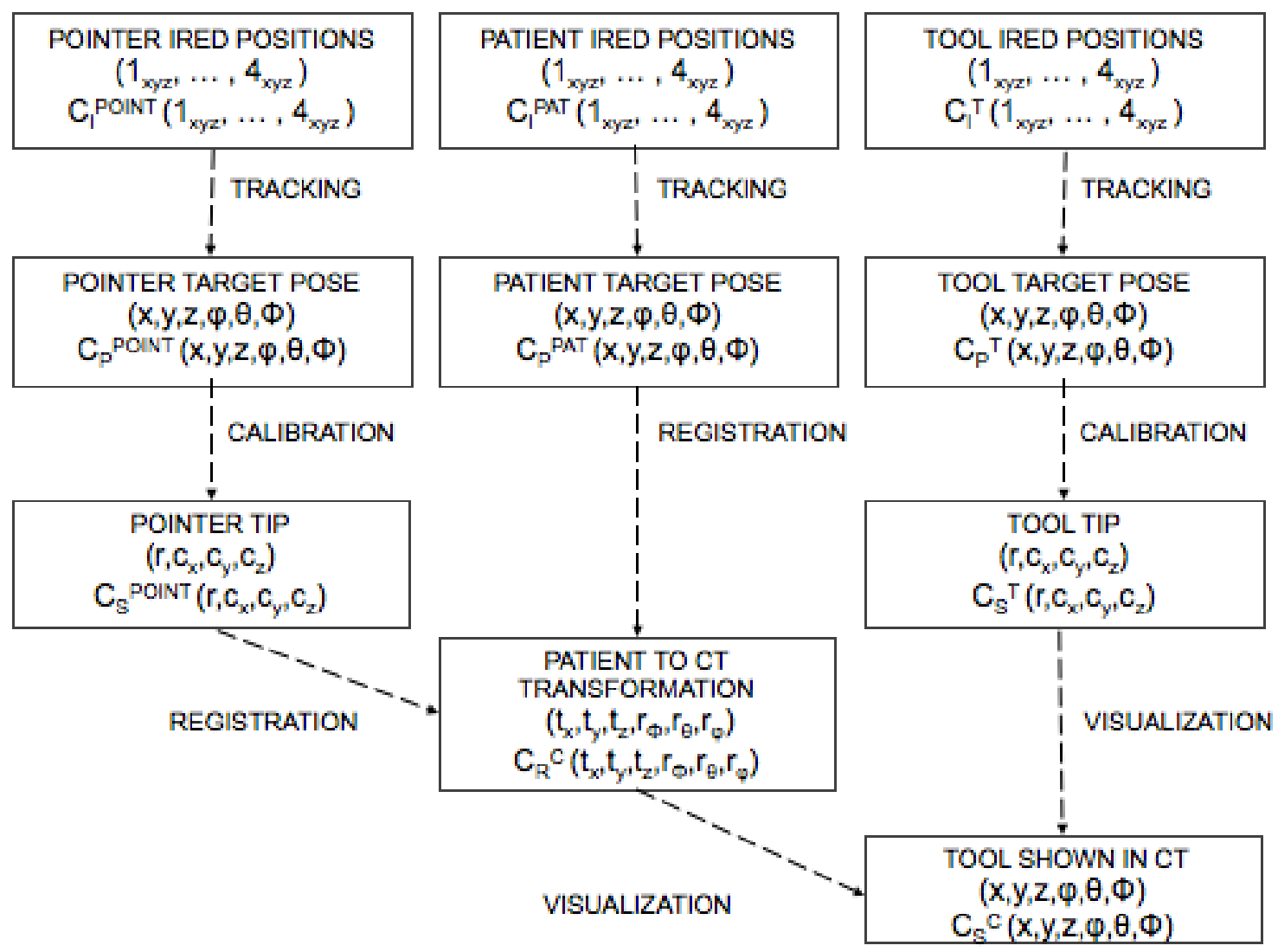

Figure 1.4: Intraoperative covariance (uncertainty) propagation of tracking, registration, and calibration measurements. (Top row) Raw IRED positions $\left(1_{x y z}, \ldots, 4_{x y z}\right)$ of the pointer, patient, and surgical tool are reported by the tracking system with corresponding covariance $C_{I}^{P O I N T}\left(1_{x y z}, \ldots, 4_{x y z}\right), C_{I}^{P A T}\left(1_{x y z}, \ldots, 4_{x y z}\right)$, and $C_{I}^{T}\left(1_{x y z}, \ldots, 4_{x y z}\right)$, respectively. (Second row) A tracking algorithm takes as input IRED positions and outputs the pose of the pointer, patient, and surgical tool target with covariance $C_{P}^{P O I N T}(x, y, z, \phi, \theta, \psi), C_{P}^{P A T}(x, y, z, \phi, \theta, \psi)$, and $C_{P}^{T}(x, y, z, \phi, \theta, \psi)$, respectively. (Third row) A calibration method takes as input the target pose and outputs the pointer and tool tip with the covariances $C_{T}^{P O I N T}\left(r, c_{x}, c_{y}, c_{z}\right)$ and $C_{S}^{T}\left(r, c_{x}, c_{y}, c_{z}\right)$, respectively. (Fourth row) The pointer tip with covariance $C_{T}^{P O I N T}\left(r, c_{x}, c_{y}, c_{z}\right)$ and the pose of the patient target with covariance $C_{P}^{P A T}(x, y, z, \phi, \theta, \psi)$ are input to the registration algorithm which outputs the transformation from the patient target coordinate frame with covariance $C_{R}^{C}\left(t_{x}, t_{y}, t_{z}, r_{\phi}, r_{\theta}, r_{\psi}\right)$. (Fifth row) The visualization step propagates the covariance of the surgical tool $C_{S}^{T}\left(r, c_{x}, c_{y}, c_{z}\right)$ and the covariance of the registration step $C_{R}^{C}\left(t_{x}, t_{y}, t_{z}, r_{\phi}, r_{\theta}, r_{\psi}\right)$ to the pose of the tool in CT coordinates with associated covariance $C_{S}^{C}(x, y, z, \phi, \theta, \psi)$. 
The second question is

- Can uncertainty be computed in the operating room?

Given the relationships depicted in Figures 1.2 and 1.4, it is easy to imagine that errors in, for example, tracking will propagate through to the calibration and registration steps. Typically, errors in these tasks are computed separately, if at all, with no thought given to how errors in one affect the other. I have proposed a method of computing intraoperative uncertainty in tracking and calibration that propagated the uncertainty through each component. I demonstrated that the estimates of uncertainty this method produced matched those from simulation and experiment.

The third question is

- How does uncertainty visualization affect surgical guidance tasks?

If we assume that intraoperative error in tracking, calibration, and registration is quite large, then it is easily conjectured that surgeons will perform better when uncertainty is shown. If, on the other hand, the surgeon is working under ideal conditions (low intraoperative error) then the question is whether uncertainty visualization can have a negative effect on surgical performance. I investigated this possibility by developing a navigation system that propagated tracking, calibration, and registration uncertainty into the tip of the surgeon's tool as the tool moved through space. I studied the effects of navigation method and uncertainty visualization type on a surgeon's ability to insert screws into the spine, performing a user study of the system with surgical residents and practicing surgeons. I demonstrated that the method of uncertainty visualization does not affect surgical performance. 


\subsection{Organization}

In Chapter 2 of this dissertation, I review the major paradigms of CAS. I briefly describe the related work in error analysis. For each CAS paradigm, I describe related visualization methods. I conclude with an overview of the field of uncertainty visualization from computer graphics research and critique these methods with respect to perceptual and cognitive theory. Chapter 3 provides a user study of the effectiveness

of a single uncertainty visualization technique which serves as the starting point of this work. Chapter 4 describes a method to compute tracking and calibration uncertainty fast enough to be computed intraoperatively. Chapter 5 describes a user study in which I applied the intra-intraoperative uncertainty model to pedicle screw insertion. I compare several methods of conveying uncertainty to a surgeon. In Chapter 6, I summarize my contributions. 


\section{Chapter 2}

\section{Review of Relevant Literature}

The literature relevant to this dissertation is described in this chapter. An overview of the major navigation paradigms in CAS is given to establish the context of the contributions. Indeed, this work in uncertainty visualization and computation assumes a navigation system that uses preoperative images for guidance; however, there are other types of systems in use today.

The most common methods of computing registration and tracking error are summarized. Methods of estimating uncertainty in registration and tracking in CAS are described.

Visualization methods used in navigation are summarized and illustrated. The types of uncertainty visualization methods in computer graphics research are classified and examples are shown of each.

\subsection{An Overview of Major Navigation Paradigms in CAS}

In this section, a broad overview of the major paradigms used in CAS is described to provide the context of this dissertation. The reader is directed to Jaramaz et al. [36] and Peters and Cleary [88] for more comprehensive treatment of the subject matter. 
The first two paradigms are navigation methods that use patient-specific anatomical information displayed on a computer screen. This information can be obtained preoperatively or intraoperatively. In both, the surgeon defines a surgical approach (a planned path) and uses the planned path to guide the position and orientation of the surgical instrument. The first two navigation types are the most common navigation systems in use [36]. The third paradigm uses no patient-specific model. The last method uses no navigation system. The position and orientation of the surgical tool is determined by a mechanical jig or guide.

\subsubsection{Navigation Using Preoperative Images}

In this model of CAS, preoperative medical images, usually from magnetic resonance imaging (MRI) or computed tomography (CT), are acquired of the target anatomy. Structures of interest are isolated from surrounding tissues by a process called segmentation. This process can be as simple as applying a threshold value (pixels of a particular value are labeled as the object in question). Segmentation is usually achieved with some human intervention because the boundaries can be difficult for a machine to distinguish. Segmentation produces a 3D patient model.

Intraoperatively, a camera system (usually optical or electromagnetic) is used to track the position and orientation of tools and the patient. Calibration brings the model of the tool and the tracked target into correspondence. Registration matches the patient's anatomy to the 3D patient model obtained preoperatively. Registration also brings any intraoperative images (e.g. fluoroscopy) into alignment. These methods require preoperative $\mathrm{CT}$ scans which are expensive and expose the patient to ionizing radiation. Intraoperative registration adds time to the procedure; however, 
navigation can reduce time required in other stages of the procedure.

There are currently several commercial navigation systems available that use preoperative images for navigation including the StealthStation (Medtronic Inc., Louisville, CO, USA), VectorVision (BrainLAB, Chicago, IL, USA), and Stryker Navigation System (Stryker, Kalamazoo, MI, USA). Figure 2.1 depicts a system developed by iGO Technologies used clinically at Kingston General Hospital. The 3D model constructed from $\mathrm{CT}$ is shown in the upper-right quadrant. The remaining quadrants are the traditional orthographic views of the anatomical planes: the transverse (top left), coronal (lower left), and sagittal (lower right) anatomical planes with the trajectory of the tool rendered as red crosshairs.

\subsubsection{Direct Navigation}

In direct navigation, patient scans are obtained during surgery. Targets are attached to the imaging device before surgery such that no intraoperative registration is required. The primary reason for using intraoperative imaging is that the images are continuously updated thus compensating for structures that move or deform. Intraoperative MRI, CT, 3D fluoroscopy are imaging modalities used for this type of navigation. The limitation of using intraoperative imaging for navigation is equipment availability, the need for a dedicated imaging device, and specialized tools (for example, intraoperative MRI requires non-metallic tools).

The StealthStation (Medtronic Inc., Louisville, CO, USA) and VectorVision (BrainLAB, Chicago, IL, USA) systems are capable of navigation with 3D fluoroscopy. The PoleStar (Medtronic Inc., Louisville, CO, USA) system uses intraoperative MRI data 


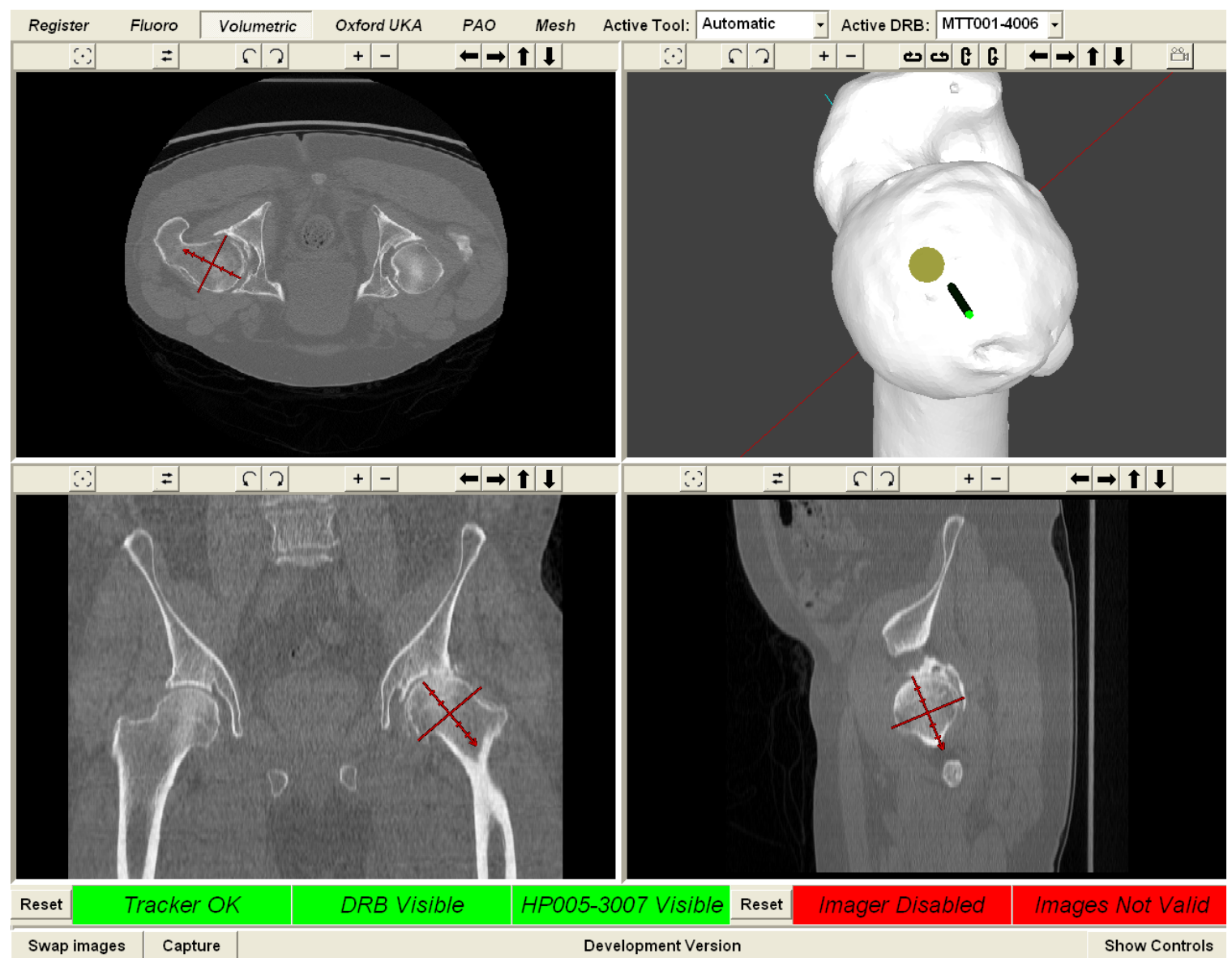

Figure 2.1: A guidance system that uses preoperative images for navigation. The upper-right quadrant shows the 3D model constructed from CT (white) and the surgical instrument (in green). The remaining views are resampled slices of the CT volume data set in the (top left) transverse, (bottom left) coronal, and (bottom right) sagittal anatomical planes. Red crosshairs indicate the current trajectory of the surgical instrument. 


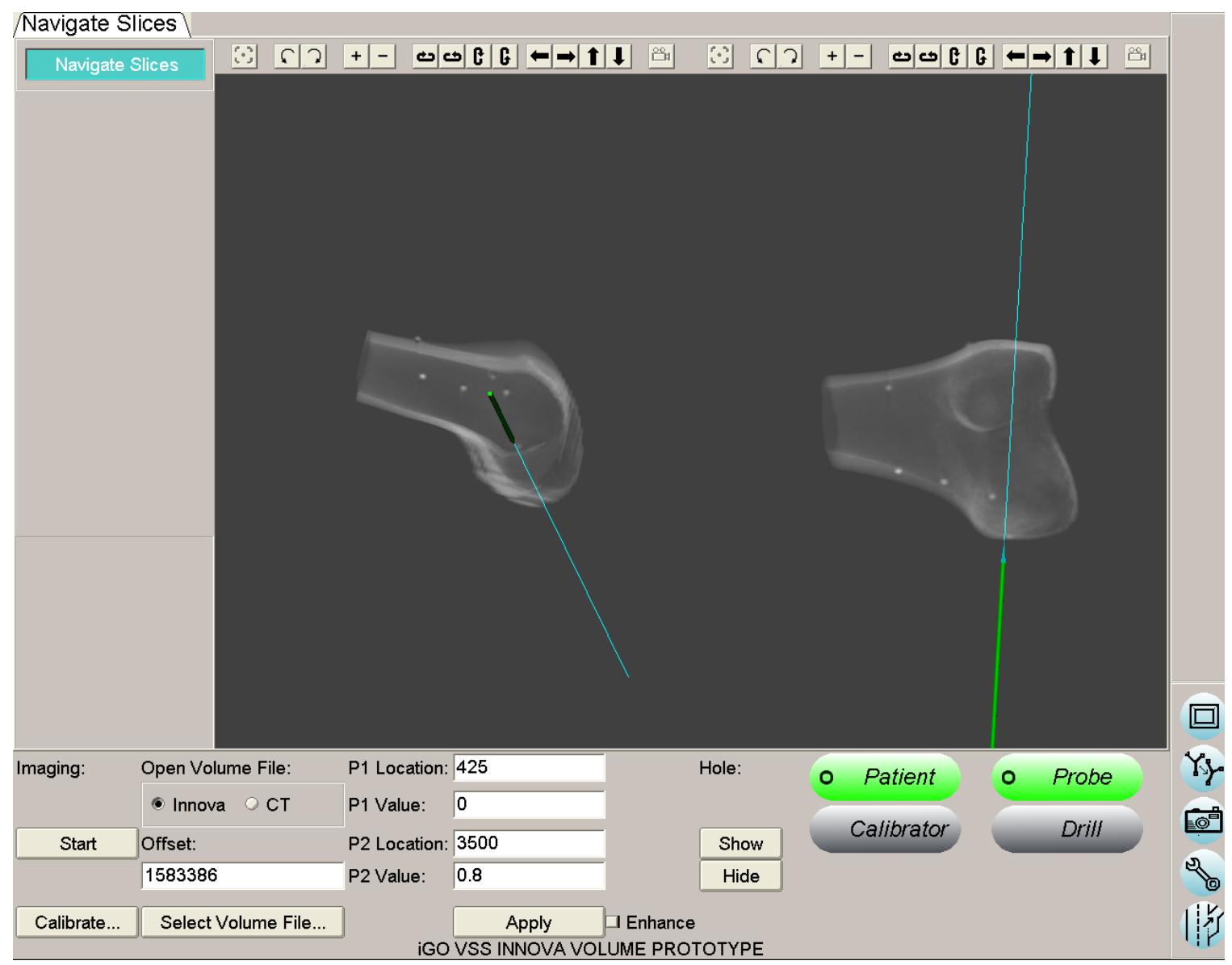

Figure 2.2: Intraoperative 3D fluoroscopic navigation system used at Kingston General Hospital. The distal femur model is rendered directly from 3D fluoroscopy. The green and cyan tubes represent the surgical tool and tool trajectory, respectively.

sets. Figure 2.2 shows the navigation system for intraoperative 3D fluoroscopic guidance used clinically at Kingston General Hospital. The volume rendered 3D fluoroscopy image of the distal femur (white) is shown with the (green) surgical tool and trajectory of the tool (cyan). 


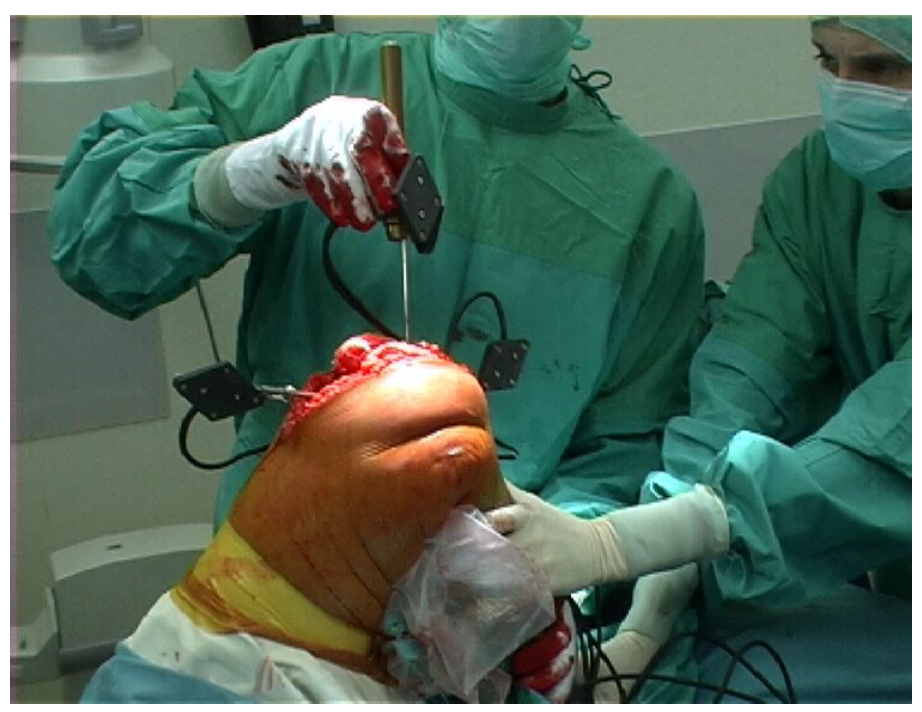

Figure 2.3: Intraoperative data collection in an image-free navigation system for total knee arthroplasty. Targets were affixed to the tibia, femur, and pointing device. [C Manuela Kunz, used with permission]

\subsubsection{Image-free Navigation}

Image-free navigation systems rely on the intraoperative collection of tracking data to define the patient's anatomy. In some systems, the shape of the anatomy is digitized using a tracked pointing device (or landmarks are simply palpated) and matched to a model constructed from several individuals [25]. In other systems, the mechanical axis is determined using the kinematics of the joints. Figure 2.3 demonstrates the intraoperative digitization of anatomy in a total knee arthroplasty. The surgeon palpated the surface of the anatomy using a tracked probe. The position of the femur and tibia were also tracked. Figure 2.4 is a screen capture of an image-free navigation system [49]. The mechanical axis of the femur and tibia was determined from the digitized points and indicated to the surgeon as yellow lines. The outline of the condyles and tuberositas are shown in blue and the epicondyles are shown in orange. 


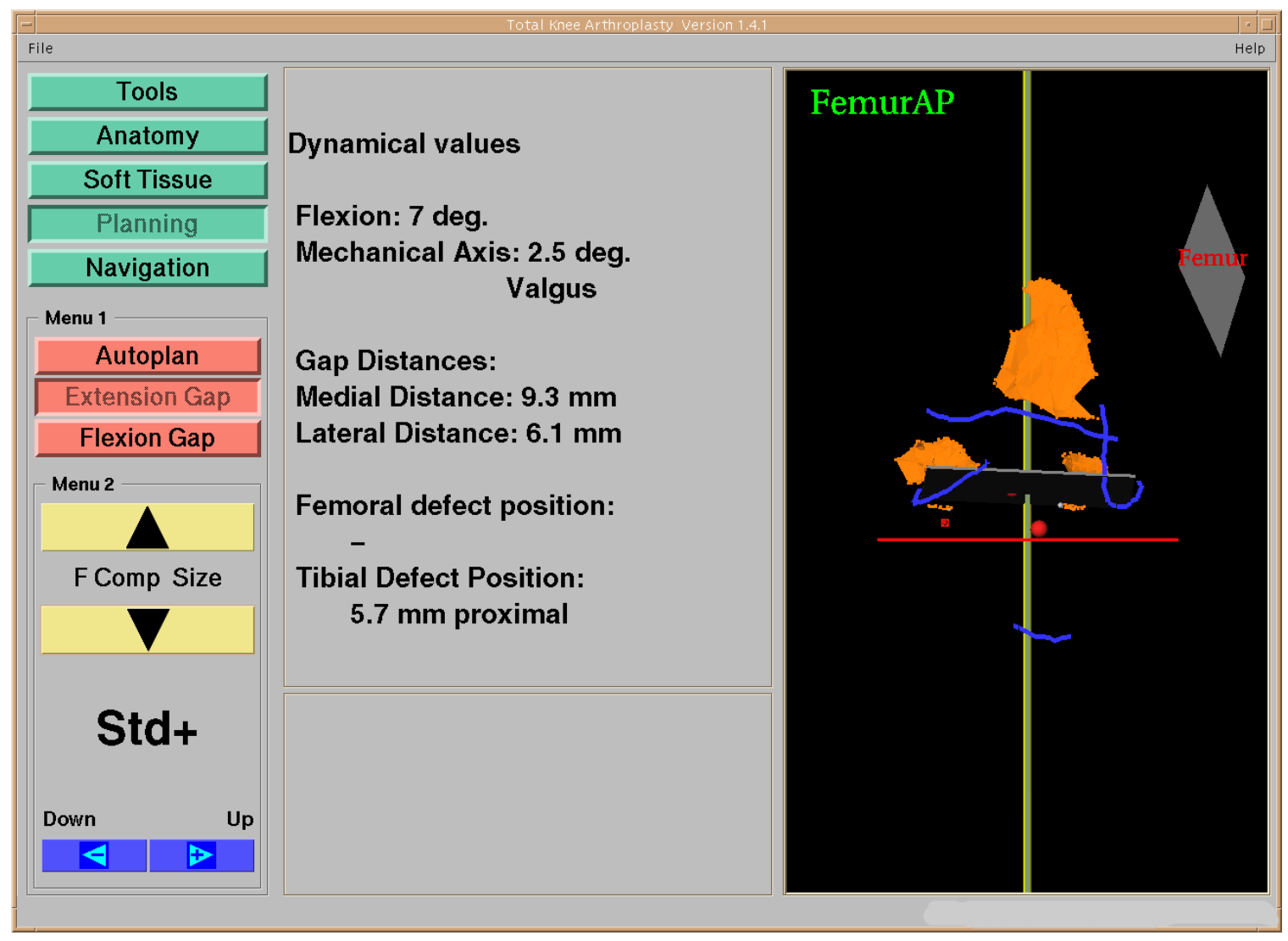

Figure 2.4: A screen capture of an image-free navigation system. The yellow line represents the mechanical axis of the femur and tibia computed from the digitized points. The outline of the condyles and tuberositas are in blue and the epicondyles are in orange. [CManuela Kunz, used with permission]

One problem with image-free navigation is that an individual's variability may not be captured within the model with which the collected data is matched; hence, the registration may be unreliable. Patient outcomes with this method vary. For example, a recent study of image-free hip arthroplasty showed mean absolute difference between measured and computed neck-shaft angles of $16^{\circ}$ [83].

Ortho-pilot (Aesculap AG, Tuttlingen, Germany) and Ci-System (DePuy Orthopaedics, Munich, Germany) are commercial image-free navigation systems. 


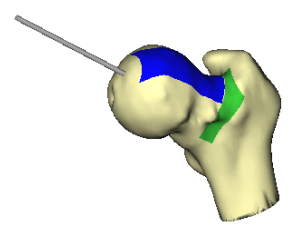

a)

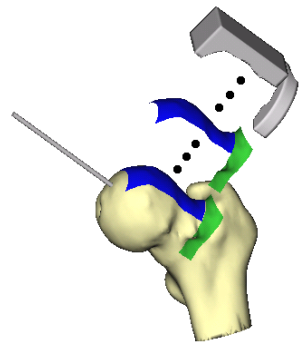

b)

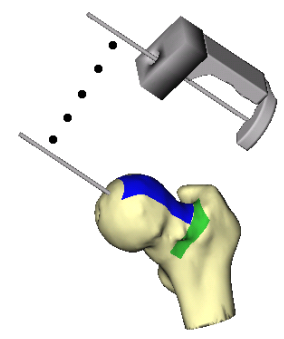

c)

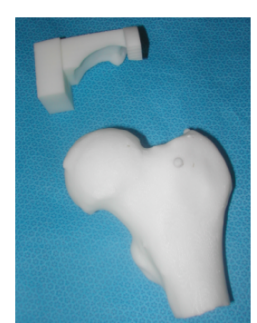

d)

Figure 2.5: Creation of the patient-specific drill template. (A) The registration surface was used to create the (B) mirror image. (C) The template was attached to the anatomy. (D) Plastic model of the femur with the template. [CManuela Kunz, reproduced with permission]

\subsubsection{No Navigation}

The final CAS paradigm to consider is methods that do not use navigation for guidance.

In the first example, patient-specific templates guided the position and orientation of surgical instruments [48]. Figure 2.5 illustrates the process of constructing a patient-specific template. First, a 3D model was created from preoperative CT. The surface of the model (that was surgically accessible) was defined by a set of points called the registration surface. The mirror image of this surface was computed using specialized software. A plastic model of the individualized template was manufactured. Intraoperatively, the surgeon clamped the template in place and used the guide to position the drill (see Figure 2.6). These templates were very easy to use but required a preoperative CT scan and the surgeon cannot change the plan intraoperatively. 


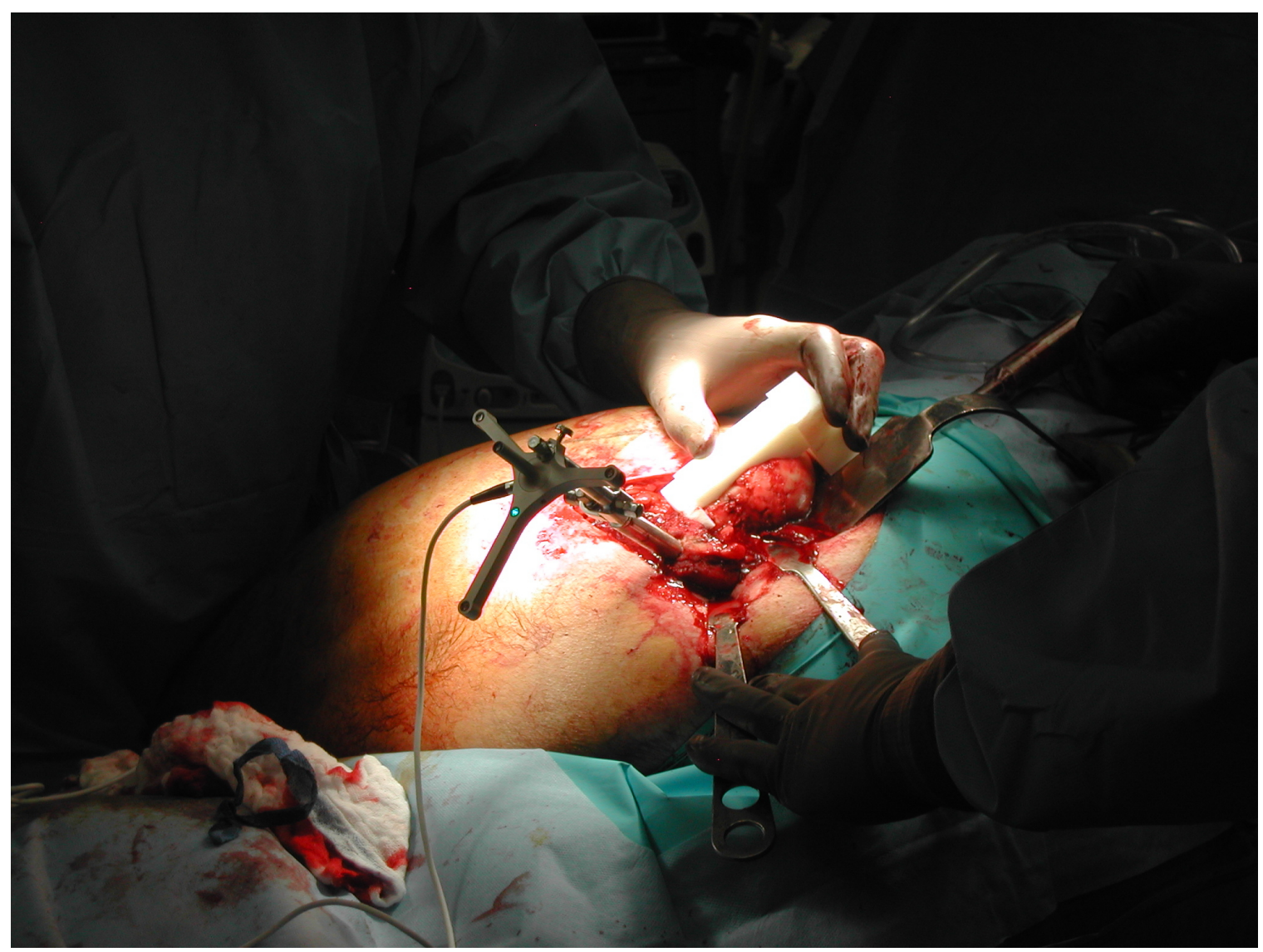

Figure 2.6: The patient-specific template was positioned on the distal femur. Note that in this particular surgery, a tracking system was used to verify the position of the template, not for guiding the position of the template. [CManuela Kunz, reproduced with permission]

The second example relates to the Taylor spatial frame, a six degree-of-freedom external fixation device for correcting bone deformities and fractures [99]. The frame consists of 6 adjustable struts and 2 circular rings in a symmetric configuration of a Stewart platform [101]. The frame is affixed to the bone with metal pins and rods and an osteotomy is performed. Postoperatively, the rings are distracted at a rate of about $1 \mathrm{~mm}$ per day and the bone responds to this mechanical stress by growing in the direction of the applied stress. Figure 2.7 illustrates a bone before 


\subsection{A CLASSIFICATION OF ERROR AND UNCERTAINTY}
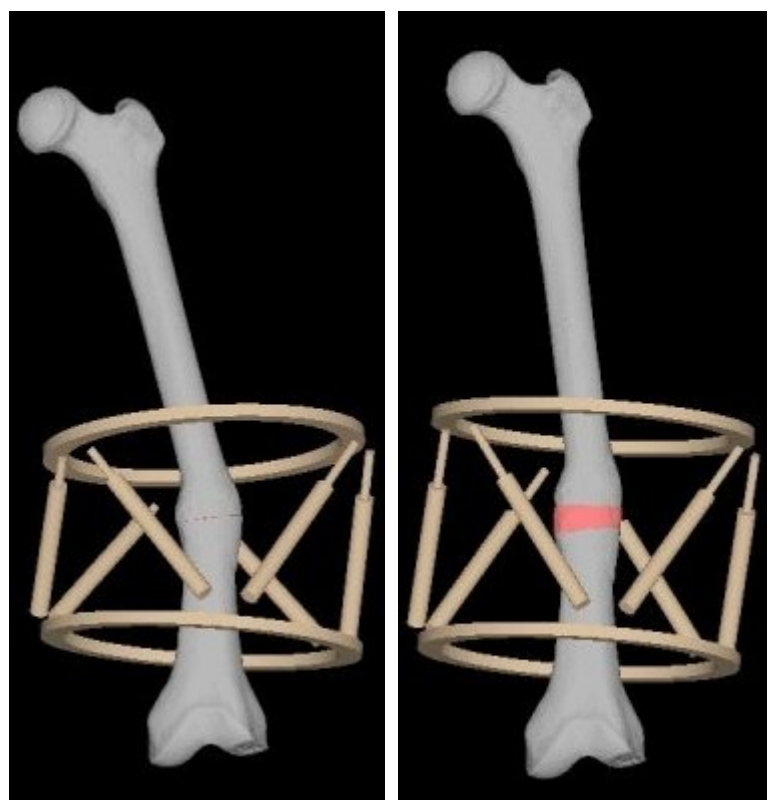

Figure 2.7: The Taylor frame (left) before and (right) after correction. By adjusting the strut lengths, the frame can be adjusted to mimic any deformity. An osteotomy is performed during surgery. After surgery, the frame is distracted over time according to a schedule.

and after correction using the Taylor frame. A computer-assist method [62] was proposed that computed the six specific strut lengths directly from CT. Figure 2.8 shows the landmarks measured from CT. This technique enabled the surgeon to make intraoperative adjustments and did not require specialized equipment in the operating room. A preoperative $\mathrm{CT}$ was required.

\subsection{A Classification of Error and Uncertainty Analysis in CAS}

In this section, error and uncertainty analysis is examined as it relates to registration and tracking in CAS. 


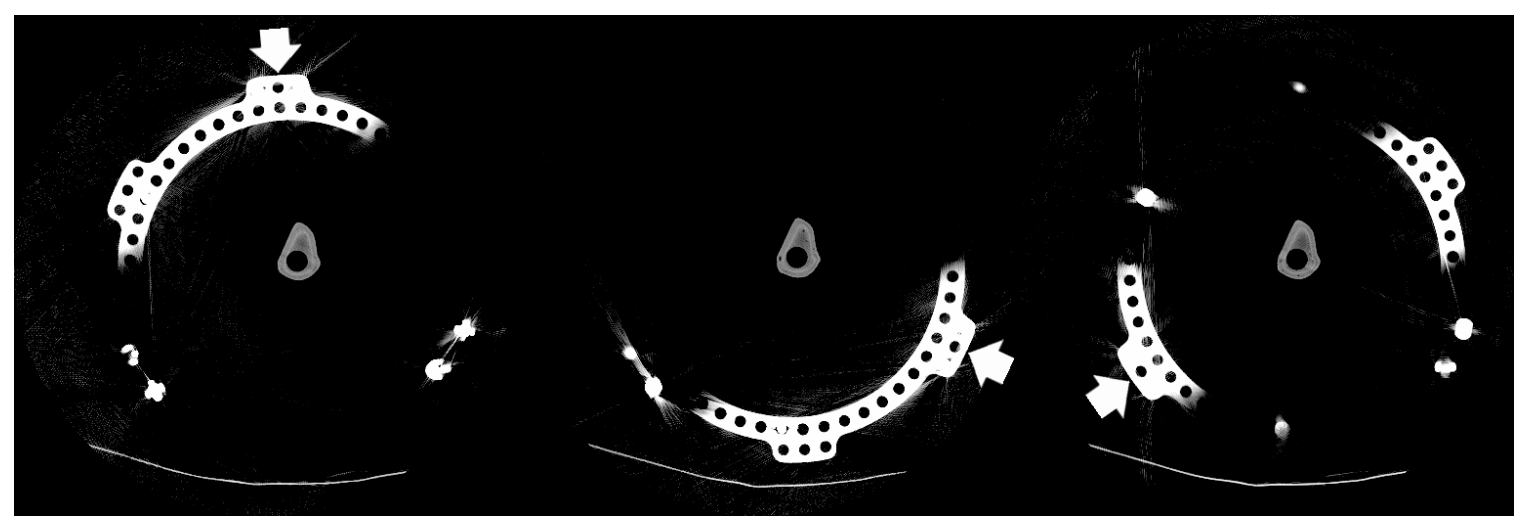

Figure 2.8: CT scan of the Taylor frame rings. The arrows point to the landmarks read directly from $\mathrm{CT}$.

\subsubsection{Registration Error}

Registration is the process of aligning the patient's anatomy (in the coordinate frame of the camera) to a 3D model (in its own coordinate frame) by computing the transformation between the two coordinate frames. Registration is a fundamental task in CAS. In orthopaedic surgery, for example, a common approach to registration is to digitize a set of points (i.e. find their 3D locations in the camera's coordinate frame) from the surface of the bone and then to find the rigid transformation that best matches the points to the $3 \mathrm{D}$ model. These points are taken by touching the bone percutaneously (through the skin) and at the site of the incision with a tracked probe. The acquired points might be displaced from the surface of the bone because of tool deflection, by simply missing the surface, or by tracking error. Registration accuracy is limited by the number and quality of points collected by the surgeon. In CAS, the success or failure of a procedure is contingent on registration accuracy. 
In orthopaedic surgery, when registering to bony anatomy, the registration transformation has six degrees of freedom (three translational and three rotational parameters) and is called rigid. Nonrigid registration algorithms align images that are deformed and therefore have more than six degrees of freedom. In this dissertation, the term registration refers to rigid registration, unless otherwise stated.

In this section, fiducial and surface-based registration is defined and registration error analysis described. Medical image registration is a richly researched area. Audette et al. [3], Lavallée [52], and Maintz and Viergever [64] review registration algorithms in more detail.

\section{Fiducial Registration}

Fiducial registration is considered the most accurate method of registration. Fiducial registration is performed using physical markers identifiable in the preoperative medical image and physically measured during surgery. These markers are characterized by the point $\mathbf{p}_{i}$ measured in the patient coordinate frame and a point $\mathbf{x}_{i}$ measured in the coordinate frame of the medical image. The fiducial registration problem (also called the absolute orientation problem) is to find the rotation $\mathbf{R}$ and translation $\mathbf{t}$ that minimizes

$$
\mathrm{FRE}=\sqrt{\frac{1}{N} \sum_{i=1}^{N}\left\|\mathbf{R} \mathbf{p}_{\mathbf{i}}+\mathbf{t}-\mathbf{x}_{i}\right\|^{2}}
$$

where $N$ is the number of markers and FRE is the fiducial registration error which is just the root-mean-square (RMS) correspondence error [59]. Two commonly cited closed-form solutions for this problem are Horn [34] and Arun et al. [2]. Orthopaedic procedures no longer use fiducial registration directly; however, surface-based registration algorithms require a solution to this problem. 
Sibson [96] demonstrated that the expected value of FRE has a chi-square distribution. Kanatani [40] fit 3D rotations to 3D data and defined the covariance matrix of rotations and analyzed the statistical behavior of the errors.

Fiducial localization error (FLE) is the difference between the measured set of fiducials and the model set of fiducials where the error can occur in either the patient or imaging coordinate frame [66]. Target registration error (TRE) is a measure of mismatch between corresponding points (that are not fiducials) after registration; typically TRE is expressed as RMS distance [24]. Simulations of TRE by Maurer et al. [66] showed that TRE was proportional to FLE, inversely proportional to the square root of the number of markers, and dependent on the configuration of markers.

FLE and TRE are both relative accuracy measures because exact fiducial locations (ground truth) are not available. Ellis et al. [20] developed a method for evaluating FLE when ground truth is unavailable and demonstrated that RMS is a poor predictor of registration error. The locations of feasible ground-truth points were used to compute alternative registrations. Worst case TRE was contingent on the distance to the centroid of the markers.

\section{Surface-Based Registration}

Surface-based registration maps points taken from the surface of the anatomy to a model of the surface. This is the most common method of registration in orthopaedic applications. The surface-based registration problem finds the rotation $\mathbf{R}$ and translation $\mathbf{t}$ that minimizes the discrepancy between the set of registration point $P=\left\{\mathbf{p}_{\mathbf{i}}\right\}$ and the model $X$. The discrepancy can be expressed as a function of the meansquared error between the registered point set $\left\{\mathbf{p}_{\mathbf{i}}\right\}$ and the nearest-neighbor point 
set $\left\{\mathbf{x}_{\mathbf{i}}\right\}$ that is a subset of $X$ :

$$
\mathrm{PRE}=\sqrt{\frac{1}{N} \sum_{i=1}^{N}\left\|\mathbf{R} \mathbf{p}_{\mathbf{i}}+\mathbf{t}-\mathbf{x}_{i}\right\|^{2}}
$$

where PRE is the point registration error [59]. The difference between Equation 2.1 and Equation 2.2 is that the corresponding model points $\left\{\mathbf{x}_{\mathbf{i}}\right\}$ are unknown and must be inferred in the equation for PRE.

Iterative Closest Point (ICP) is a popular method proposed by Besl and McKay [7] for minimizing Equation 2.2. The algorithm consists of three stages: 1) correspondences are established between the model and the object based on proximity, 2) the rigid transformation is estimated that minimizes the distance between each pair of corresponding features in the model and object, and 3) the transformation is applied to all features. These steps continue until convergence is reached. The algorithm requires a good initial estimate. Many variants of ICP exist (see Audette et al. [3], Lavallée [52], and Maintz and Viergever [64] for complete references).

\subsubsection{Registration Uncertainty}

Methods of computing registration uncertainty are discussed in this section. These methods are categorized as analytic, filter, and iterative closest point and related work in CAS is described within each category. 


\section{Analytic Methods}

The idea of analytically computing and propagating covariance estimates is not new. The covariance matrix

$$
C O V(u, v)=\sigma_{u v}^{2}=\langle(u-\bar{u})(v-\bar{v})\rangle
$$

where \langle\rangle denotes the expected value and $\bar{u}$ and $\bar{v}$ the respective means. The expected value for a single variable is defined as

$$
\langle f(x)\rangle=\sum_{x} f(x) P(x)
$$

where $P(x)$ is the probability density function. For a single continuous variable, the probability density function is

$$
\langle f(x)\rangle=\int f(x) P(x) d x .
$$

The probability density function is the derivative of the distribution function. The distribution function describes the probability that a variable takes a value less than or equal to a given number [113].

The propagation of covariances where $x=f(u, v)$ is assumed to be that of Bevington [8]:

$$
\sigma_{x}^{2}=\sigma_{u}^{2}\left(\frac{\partial x}{\partial u}\right)^{2}+\sigma_{v}^{2}\left(\frac{\partial x}{\partial v}\right)^{2}+2 \sigma_{u v}^{2}\left(\frac{\partial x}{\partial u}\right)\left(\frac{\partial x}{\partial v}\right) .
$$

Hoff and Vincent [33] used this method to compute pose covariance in a headmounted display system. Sielhorst et al. [97] applied the same methodology to estimate the accuracy of a tracked surgical instrument by modeling the geometry and 
visibility of targets. Target pose was computed using least-squares optimization of fiducials and propagated to TRE.

Ma et al. [59, 61] derived an equation that predicts TRE for fiducial and surfacebased registration with anisotropic noise. Simulation results used an unscented Kalman filter fiducial registration algorithm that achieved TRE corresponding to the derived equations. Tests with real data were reported by the authors in later work [74]. The authors also demonstrated that several known estimators were theoretically equivalent. Ma et al. [61] reported that, because Sielhorst et al. [97] used a least-squares optimization (which assumed isotropic noise), the covariance was underestimated.

Shamir et al. [94] demonstrated that the analytic methods based on computed FRE may underestimate actual covariances and that a reliable TRE estimator was needed.

Bromiley et al. [11] derived a covariance of mutual information registration parameters (translation, rotation, scaling) of MRI scans of the brain. They validated their results by comparison with Monte Carlo simulations.

\section{Filtering Methods}

Kalman filtering methods estimate the mean and covariance of the true system state from noisy measurements. A good feature of the Kalman filter is that it provides fast, highly accurate solutions suitable for real-time applications. Appendix A describes Kalman filtering for nonlinear, non-Gaussian filtering with an emphasis on covariance estimation. Special attention is paid to Kalman filtering because this dissertation proposes a method of computing intraoperative uncertainty in registration, tracking, 
and calibration based on Kalman filtering in Chapter 4 .

Pennec and Thirion [87] used frame (or local coordinate frame) correspondences (instead of point to point correspondences) to predict and verify rigid registration covariances. The covariance estimation was performed using an extended Kalman filter. Pennec et al. [86] also extended their model to pose estimation and validation.

Ma $[60,59]$ described a particle filter that estimated the mean and covariance of the surface-based registration parameters using a Kalman filtering-based approach. The uncertainty estimates were verified analytically in later work [61].

\section{Iterative Closest Point Methods}

Stewart [100] proposed incorporating error covariance matrices estimates into pointbased registration using ICP. The author generalizes ICP to use Mahalanobis distance in the matching process. The Mahalanobis distance is a distance metric computed using the mean and covariance matrix instead of the traditional Euclidean distance metric. The measurement error was isotropic but could be concentrated in a single direction. The author does not present simulation results.

Stoddart et al. [102] extended Pennec and Thirion [87] to surface-based registration by adding a registration index to ICP. This uncertainty metric described how well two surfaces match in the registration and assumed that the noise was isotropic. No validation of the uncertainty was described.

\subsubsection{Tracking Error}

The tracking problem determines the position and orientation of an object as it moves through space. In CAS, a tracking system measures the 3D position of markers 
attached to targets with known geometry. The tracking system is usually either electromagnetic $(\mathrm{EM})$ or optical. The system reports raw $3 \mathrm{D}$ position and or $6 \mathrm{D}$ position and orientation data.

Attempts have been made at computing error in tracking systems by empirical study. Dietz et al. [15] computed the uncertainty in optical camera geometry and studied the effect on biplanar fluoroscopic images. A calibration device with known geometry with simulated and real data validated the uncertainty model.

Frantz et al. [28] proposed determining accuracy by moving an object of known geometry throughout the measurement volume (a standard method of determining measurement volume error). The object's true position was measured with an Optotrak system and coordinate measurement machine (both by Northern Digital Inc., Waterloo, $\mathrm{ON})$.

Nafis et al. [75] computed the dynamic accuracy and distortion of EM systems by 'scribbling' with an EM-tracked pointer on a precision granite block. Feuerstein et al. [23] computed dynamic errors by comparing the poses computed by an optical tracking system and the EM system. The system was corrected if the magnitude of the error became too great. EM tracking system vendors are interested in assessing tracking error because EM systems are susceptible to field distortion from some metal objects.

\subsubsection{Tracking Uncertainty}

Deriving an analytic expression for tracking uncertainty is analogous to finding the distribution of FLE. Hence, the methods described in Section 2.2.2 for computing FLE can be used to compute tracking uncertainty. 


\subsection{An Overview of Visualization in CAS}

Reconstruction of a 3D model from a medical image is a key step in surgical guidance. For each of the three navigation paradigms that were discussed in Section 2.1, methods for constructing 3D models from 2D image slices are described and examples are shown of visualizations from navigation systems. For an in depth discussion of the much broader topic of medical visualization, refer to the survey by Johnson and Tricoche [37].

\subsubsection{Navigation Using Preoperative Images}

In the first navigation paradigm, patient scans are performed prior to surgery. The construction of 3D models is performed using a process called segmentation.

\section{Segmentation}

The simplest model of 3D reconstruction extracts contours from 2D slices that are stacked creating the 3D model. The extraction process is called segmentation. Medical image segmentation is a challenging task due to the difficulties of distinguishing the boundaries between anatomical features in medical images. Segmentation has been a topic of research for over two decades. In this section, the primary concern is with rendering 3D models after segmentation is performed. For details of image segmentation algorithms, the reader is directed to surveys by Duncan and Ayache [17], Freixenet et al. [29], McInerney and Terzopoulos[69], Noble and Boukerroui [78], Zhang et al. [118], and Zhang [119].

Reconstructing models using segmentation produces clean polygonal models for rendering and registration. Figure 2.9 is a screen capture of the preoperative planning 


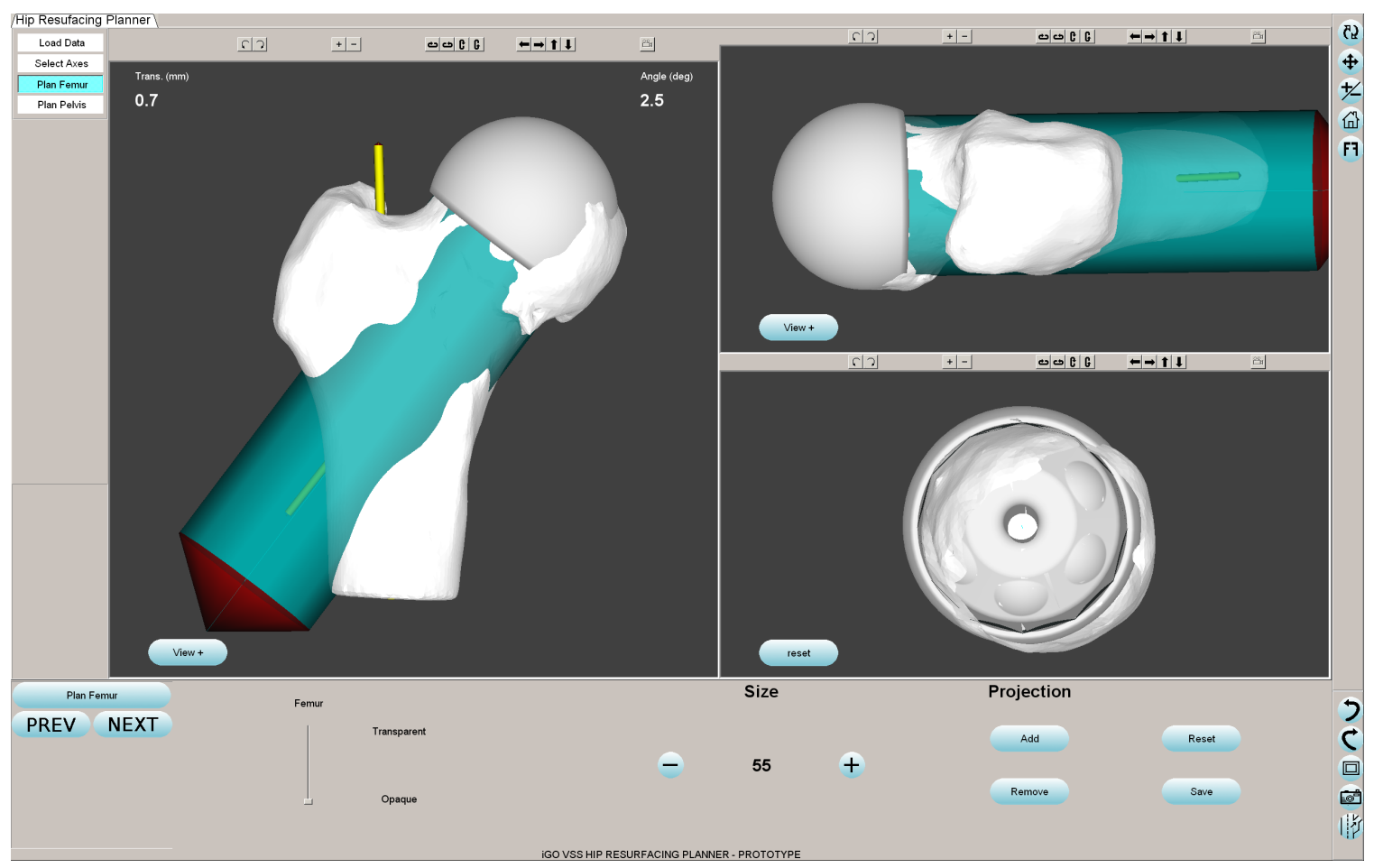

Figure 2.9: A screen capture of the three anatomical views of the preoperative planning stage of a hip resurfacing. The 3D model of the femur constructed from CT data is shown in white. The green cylinder represents the planned orientation of the grey hip resurfacing component.

software used by Kingston General Hospital for hip resurfacing. The screen shows the distal femur (hip) constructed using segmentation of CT data. The green cylinder represents the planned orientation of the hip resurfacing component.

\subsubsection{Direct Navigation}

Direct volume rendering and isosurface extraction are two techniques for automatically constructing 3D reconstructions of medical image data for use in direct navigation systems. 


\section{Direct Volume Rendering}

Direct volume rendering is a technique for viewing three-dimensional data as twodimensional images. Elements of the volume can be automatically extracted by specifying the transfer function that maps the data to properties such as opacity and color. The display properties are determined without interference by an operator; hence data set values are directly mapped to the two-dimensional image [37]. The right transfer function must be found to highlight the desired elements of the data. Specifying the transfer function can be difficult due to the complexity of the parameters that control rendering. Kindlmann and Durkin [45] developed a method of semi-automatically generating transfer functions by computing first and second directional derivatives of the gradient.

The Advantage Workstation (GE Healthcare, Milwaukee, USA) is a system that post-processes 2D, 3D, and 4D data sets directly from CT/MRI. The manufacturer has predefined settings that implement the ideal transfer function for a specified task. Figure 2.10 is a screen capture of a direct volume rendering reconstruction from Advantage Workstation of two cadaver feet from CT. This system is used clinically by Kingston General Hospital.

\section{Isosurface Extraction}

Isosurface extraction is the process of separating regions of higher and lower values from a volume data set [37]. In medical image data sets, this extraction can automatically segment anatomical structures of interest. Isosurface extraction and segmentation require an extraction step. Lorenson and Cline [57] introduced the 


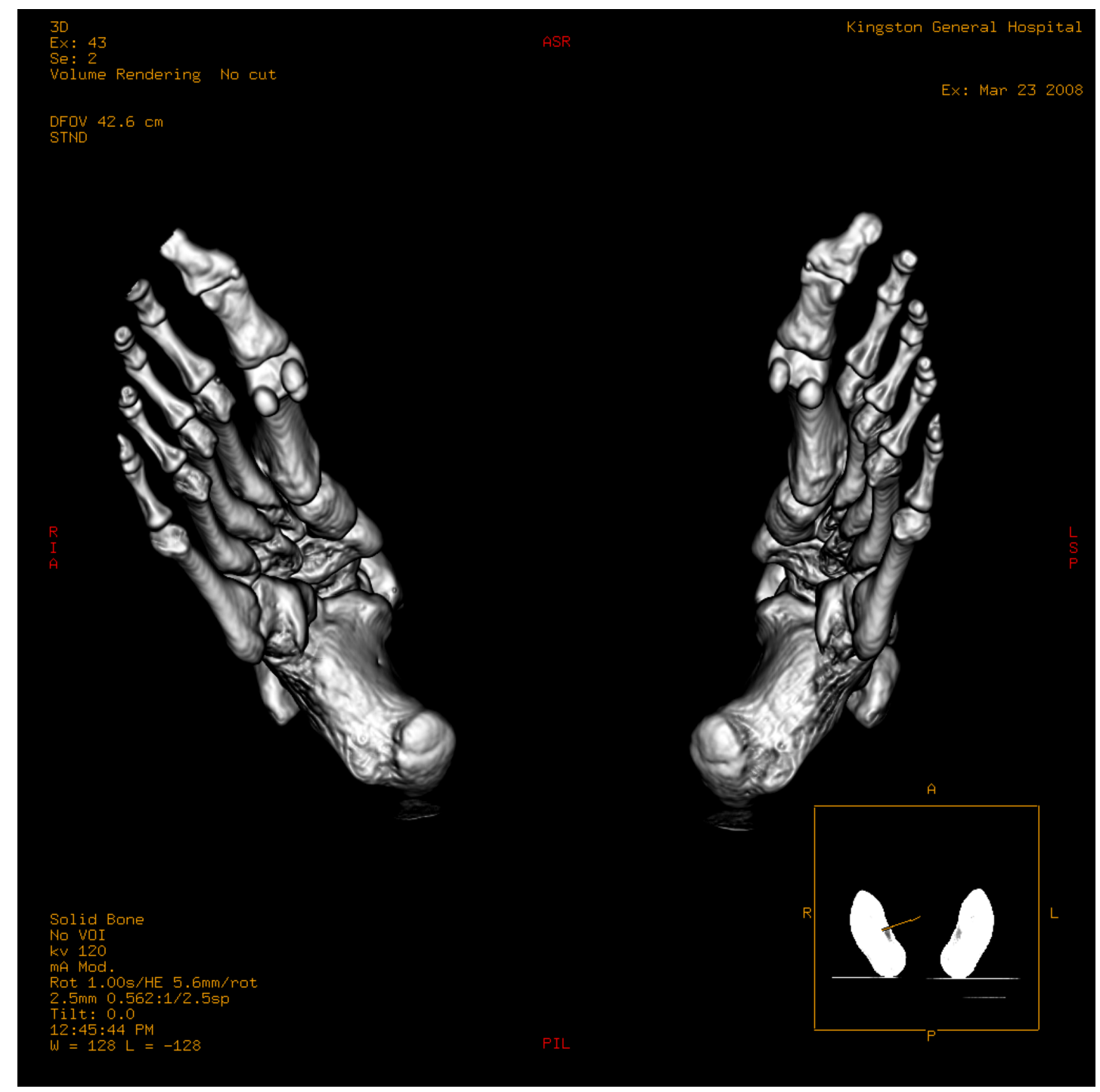

Figure 2.10: A screen capture of cadaver feet reconstructed from CT using Advantage Workstation (GE Healthcare, United Kingdom). The system has predefined transfer functions for specific anatomical structures. 

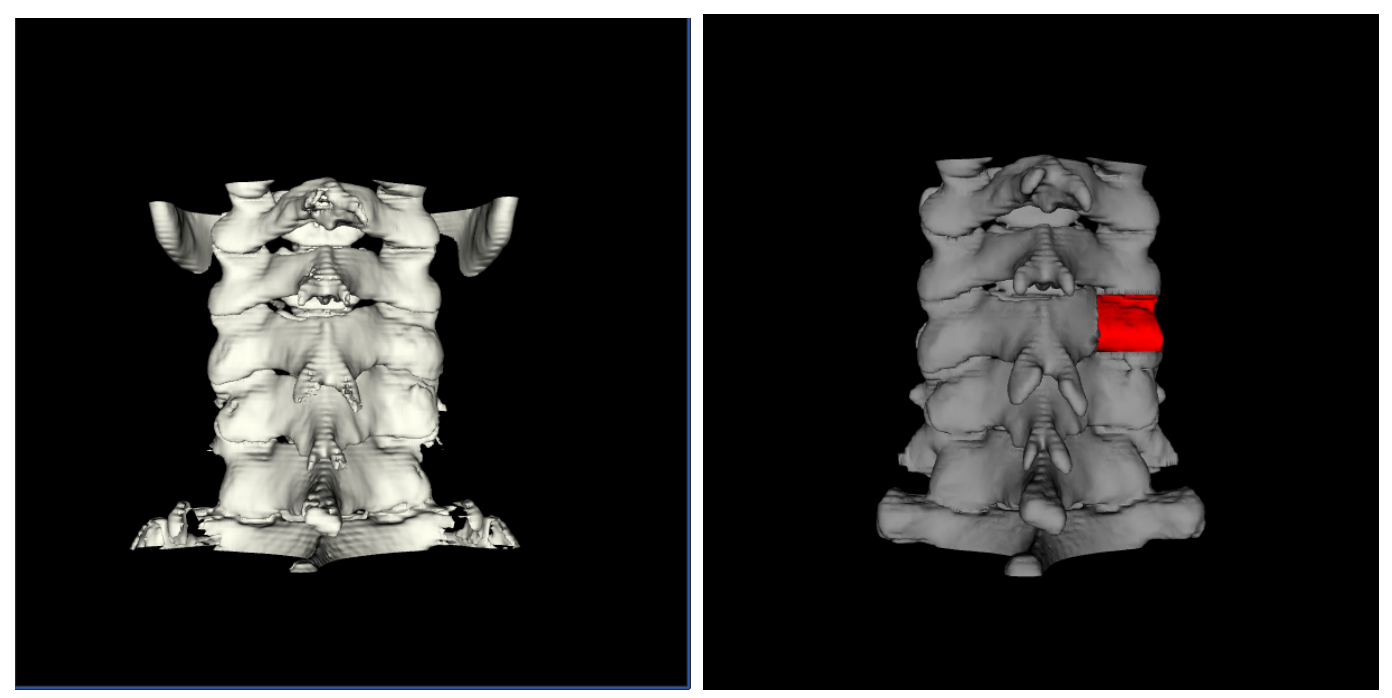

Figure 2.11: Comparison of isosurface extraction and segmented model. (Left) Isosurface extraction of the lumbar spine from CT data using the Visualization Toolkit implementation of marching cubes. (Right) A 3D surface model constructed from segmentation of the same data set. Note that the isosurface rendering is courser in appearance.

marching cubes algorithm which has become the most widely used isosurface extraction method. Figure 2.11 depicts a model of the lumbar spine constructed by isosurface extraction and segmentation. The isosurface model was produced using the marching cubes implementation in the Visualization Toolkit (Kitware Inc., New York, USA). Optimal isovalues are usually chosen by trial and error. The difficulty of choosing the right isovalues is apparent if the isosurface model is compared with the manually segmented model. The isosurface model contains many more surfaces for registration than the segmented model. 


\subsubsection{Image-free Navigation}

Image-free navigation systems do not use preoperative images for constructing patient models. Instead, a static model of anatomy is constructed from several individuals. Because the library is built in advance, there is some variability in the type of visualization. For example, in Figure 2.4, no model of the patient anatomy was rendered. In Figure 2.12, a screen capture of the Ci Knee Essential commercial image-free system (DePuy Orthopaedics, Munich, Germany) is shown. Note that the 3D rendering of the bone was not patient specific.

\subsection{Related Work in Uncertainty Visualization}

Designing and evaluating good visualizations requires an understanding of the perceptual cues and cognitive tasks that humans use to integrate information. I describe general cognitive and perceptual theories from Bertin, Tufte, and Ware and apply these theories to six categories of uncertainty visualizations.

\subsubsection{Perceptual and Cognitive Theory for Visualization Design}

In the following, I describe the perception work of Bertin [6], Tufte [105], and Ware [112] as it relates to the design and evaluation of visualizations.

Bertin Bertin [6] established a theoretical foundation for information visualization based on practical experience as a cartographer. A graphic designer manipulated a set of visual variables in two dimensions that can be applied to a point, line, or area. These variables were size, value (degrees between white and black), texture, color, orientation, shape, and the two dimensions that define a plane. The visual 


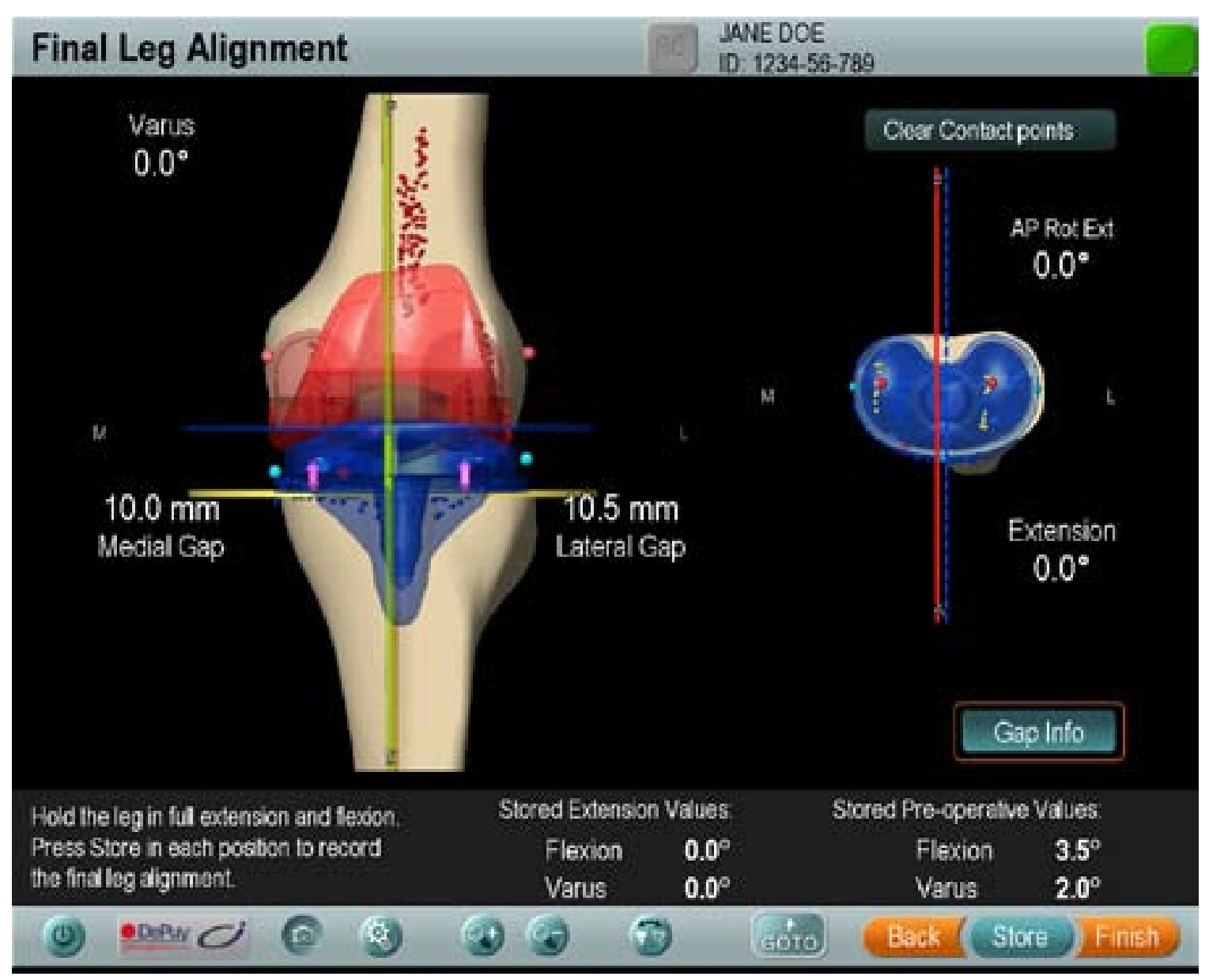

Figure 2.12: A screen capture of the Ci Knee Essential [14] navigation system for total knee arthroplasty. The 3D patient model was constructed from a library of individuals. [@DePuy Orthopaedics]

variables had different capacities for representing information. Each varied by level of organization and length. The level of a variable can be selective (easily isolated from other objects), associative (easily grouped with similar objects), ordered (in a defined order), and quantitative (distance between each category can be expressed numerically). Every combination of visual variable, level of organization, and length were demonstrated graphically by Bertin. Bertin provided many specific rules for 
constructing graphics. Some examples included:

- There is an optimum number of visible marks that can be contained in a figure which is called graphic density. Legibility is effected by this density.

- The perception of angles diminishes as the angle approaches 0 or 180 degrees. The scale of a graphic should be determined by the legibility of the angles.

- Shapes must be larger than $2 \mathrm{~mm}$ to be legible. Dashes, points, and crosses are the only legible shapes under $2 \mathrm{~mm}$. A dash must be four times its width.

- The optimal amount of black in a graphic is between $5 \%$ and $10 \%$. If there is too much or too little, a density adjustment should occur.

- The background should not be more visible than the subject matter.

This work was based on the idea that the visual system has three available sensory variables: variation of marks and two dimensions in the plane. The visualization must be able to communicate instantly the relationship between these three variables.

As Ware [112] recognized, most of Bertin's work was based on his own experience and judgment. No theories of perception or scientific studies were referenced.

Tufte Tufte [105] proposed general principles of graphical excellence and integrity that lead to good visualizations. Tufte described graphical excellence as "that which gives to the viewer the greatest number of ideas in the shortest time with the least amount of ink in the smallest space" and most interesting to uncertainty visualization, "graphical excellence requires telling the truth about data"(Tufte [105], pg. 51). Toward that end, Tufte specified guidelines: avoid distorting the meaning of data, allow the data to be seen at varying levels of detail (from an overview to fine detail), 
present large amounts of data in a small space, and integrate the data with statistics and other descriptions of the data. In order to reveal data and ensure graphical integrity, he argued six principles (Tufte [105], pg. 77):

1. Graphical representations of numbers should be proportional to the magnitude of represented numerical quantities.

2. Text should be used to avoid ambiguity.

3. Data variation, not design variation, should be shown.

4. Monetary units should be shown as standardized units. (The implication for other applications is that the data should be normalized.)

5. The number of depicted dimensions should not be larger than the data dimensions.

6. Data should not be shown out of context.

In the data-ink maximization principle, Tufte suggested that the largest amount of data should be shown with the least amount of ink. The basic idea was that all of the ink in a graphic should have a reason. Data density was a practical measure of the amount of data elements divided by the area of the graphic. If this number was low, the graphic should be reduced in size.

Ware Ware [112] combined research (his own and others) from physiology, perceptual, and cognitive psychology into a textbook on the science of visualization. The research presented supports many of theories of Bertin and Tufte with empirical data from user studies. Ware offered guidance on choosing lightness, brightness, contrast, 
constancy, and colors in visualization based on perceptual issues which agreed with Bertin's principles. The low-level channels in our vision system let us distinguish color, position, simple motion, and stereoscopic depth (similar to Bertin's visual variables). If we wished to differentiate data dimensions, each value must be mapped to a different channel.

Ware suggested that eight Gestalt laws of pattern perception can be applied to visualization design (Ware [112], pgs. 189-196):

1. spatial proximity (objects that are close together are grouped together perceptually),

2. similarity (similar objects are grouped together),

3. connectedness (connected objects are grouped together),

4. continuity (smooth and continuous lines are easier to distinguish than sharply changing ones),

5. symmetry (objects arranged symmetrically are easier to perceive),

6. closure (objects with open contours are perceived as closed),

7. relative size (smaller parts of a pattern are perceived as separate objects), and

8. figure and ground (figure is perceived in the foreground and ground is behind the figure).

Ware's principles moved from the two dimensional graphics of Bertin and Tufte to three dimensional visualizations (and four dimensions if time is considered a dimension). He argued that a $3 \mathrm{D}$ visualization must still be presented as a clear 2D 
image. Perception in 3D presents additional challenges related to depth cue theory (Ware [112], pgs. 260-272):

- Perspective cues: The true size of an object is perceived instead of the size of the object in the image plane. Depth cues as a result of perspective are parallel lines converging to a point and objects in the distance appearing smaller than objects that are closer.

- Pictures seen from the wrong viewpoint: Distortion occurs because the picture is not viewed from the correct viewpoint but, after a few minutes, we no longer perceive this distortion.

- Occlusion: An object that overlaps another object is perceived as closer.

- Depth of focus: Highlighting objects can be achieved by blurring unimportant objects because this mimics how our visual system distinguishes background and foreground.

- Cast shadows: Shadows help localize an object with respect to a surface or another object in a scene. Changes in the direction of a shadow can show motion.

- Shape-from-shading: Simple lighting models are more effective in conveying surface shape because our brains generally assume that lighting manifests from above.

- Eye accommodation: Our eyes focus on objects and can use this information to determine the size of close objects but not to determine distance. 
- Structure-from-motion: Structure-from-motion helps us determine the layout of objects in space and the 3D shape of objects.

- Eye convergence: Our brains cannot easily compute the distance to a fixed object unless the object is within arm's reach. (We can recalibrate quickly if other information is available.)

- Stereoscopic depth: The brain receives slightly different information from each eye that must be resolved into a single image.

Ware described low-level tasks that must be considered when designing effective visualizations. For example, a person who is positioned at a computer requires approximately $130 \mathrm{~ms}$ to react to a visual cue. A person selecting an object on the screen using an input device such as a mouse or joystick requires a fixed amount of time that has been measured empirically by researchers.

Ware argued that all of these factors (perceptual issues, aesthetic principles, depth cues, and interaction rules) must be understood to create visualizations that are good visual representations that can be interacted with in an effective way.

\subsubsection{Uncertainty Visualization Methods}

In this section, the state-of-the-art in uncertainty visualization techniques is reviewed. The classification of the methods is adapted from Pang and colleagues [85]. What follows is not an exhaustive list, but rather samples from each category of uncertainty visualization. Uncertainty visualization methods can be classified as adding glyphs, adding or modifying geometry, modifying attributes, using animation, displaying confidence information, or representing uncertainty with charts and graphs. 

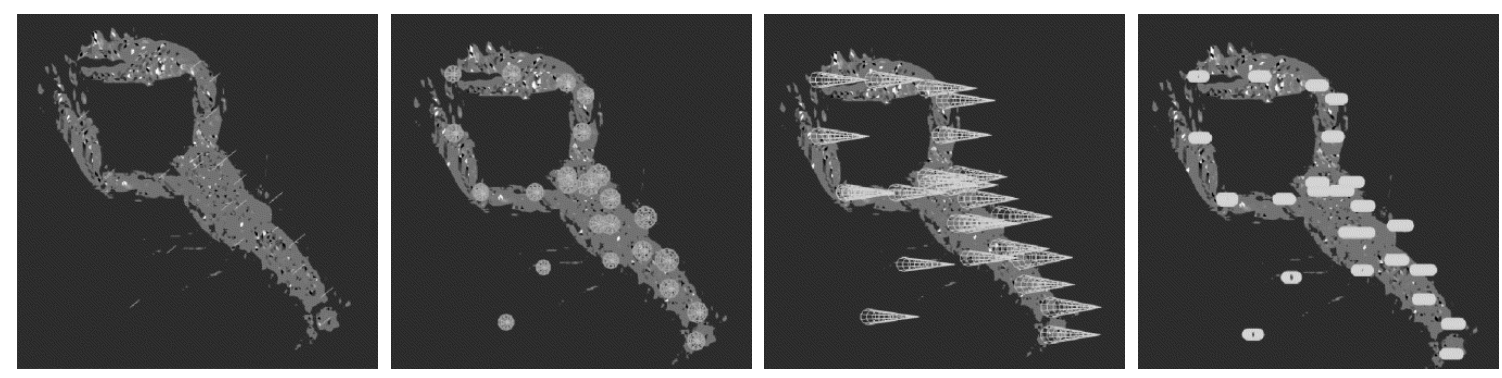

Figure 2.13: Example glyphs representing uncertainty: (a) arrow, (b) ball, (c) cone, and (d) cylinder glyphs. The size and orientation of the glyph represents the amount and direction of uncertainty [77]. [C2004 Elsevier]

I give examples of each class of uncertainty visualization, and when available, an example is drawn from medical visualization. Each method is briefly evaluated with the ideas of Bertin [6], Tufte [105], and Ware [112].

\section{Add Glyphs}

Glyphs are icons such as cones, points, balls, or arrows that indicate uncertainty through shape or color. For example, in Figure 2.13, uncertainty is rendered as arrows, balls, cones, and cylinder glyphs [77]. Figure 2.14 shows cones representing the $95 \%$ confidence angle of the level of the splenium in the corpus callosum of the brain [38]. Figure 2.15 shows EEG data marked with circles and squares that represent areas of high uncertainty [42]. Hoff and Vincent [33] represented pose uncertainty using cone (orientation) and ellipse (position) glyphs. Figure 2.16 shows their visualization method applied to head pose accuracy in augmented reality systems.

Glyphs are prime examples of manipulating Bertin's visual variables. Color, size, value, texture, orientation, and shape can all be varied. A designer of glyphs should 


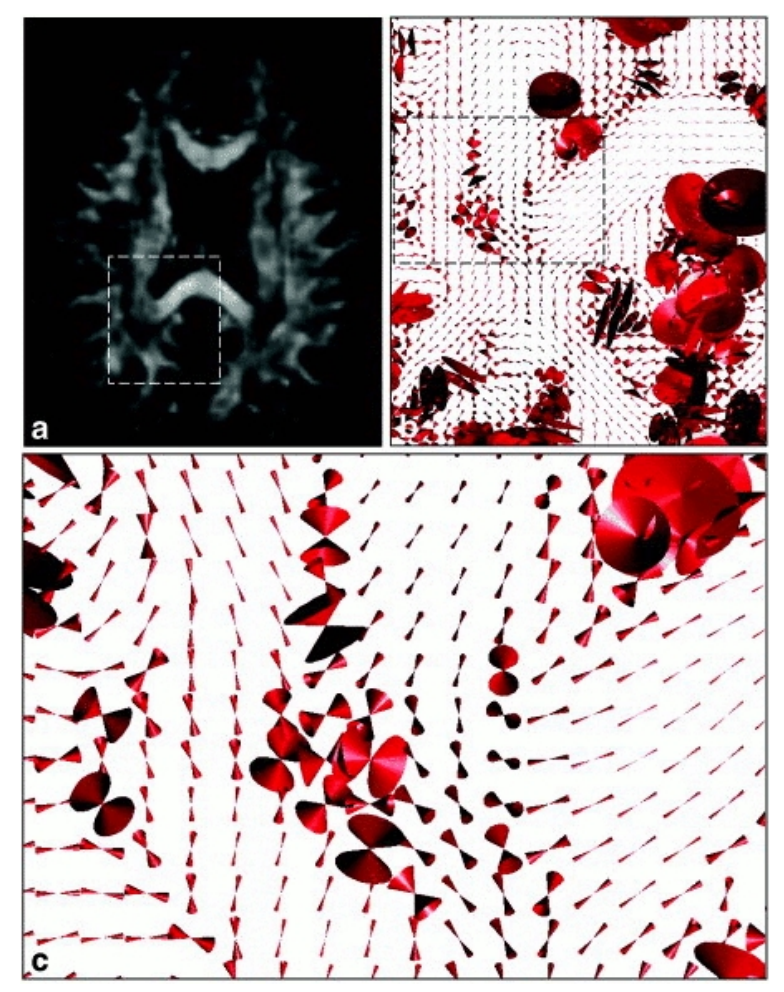

Figure 2.14: Uncertainty cones representing the $95 \%$ confidence angle of the level of splenium in the corpus callosum of the brain [38]. [@2003 Wiley]

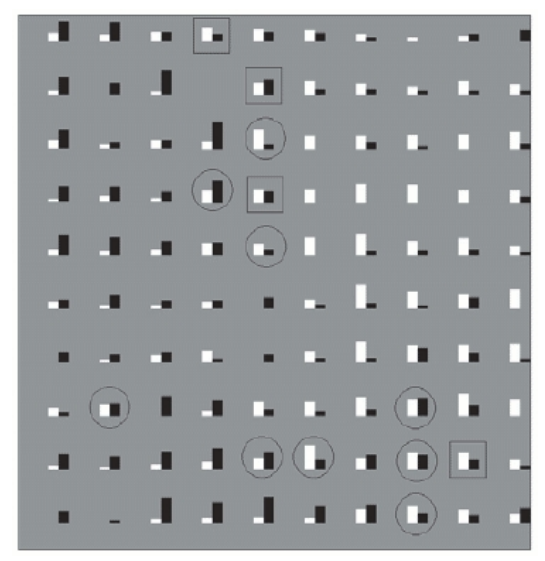

Figure 2.15: Representation of uncertainty in EEG data of neonatal seizures. Circles and squares indicate categories of high uncertainty [42]. [C2004 IEEE] 

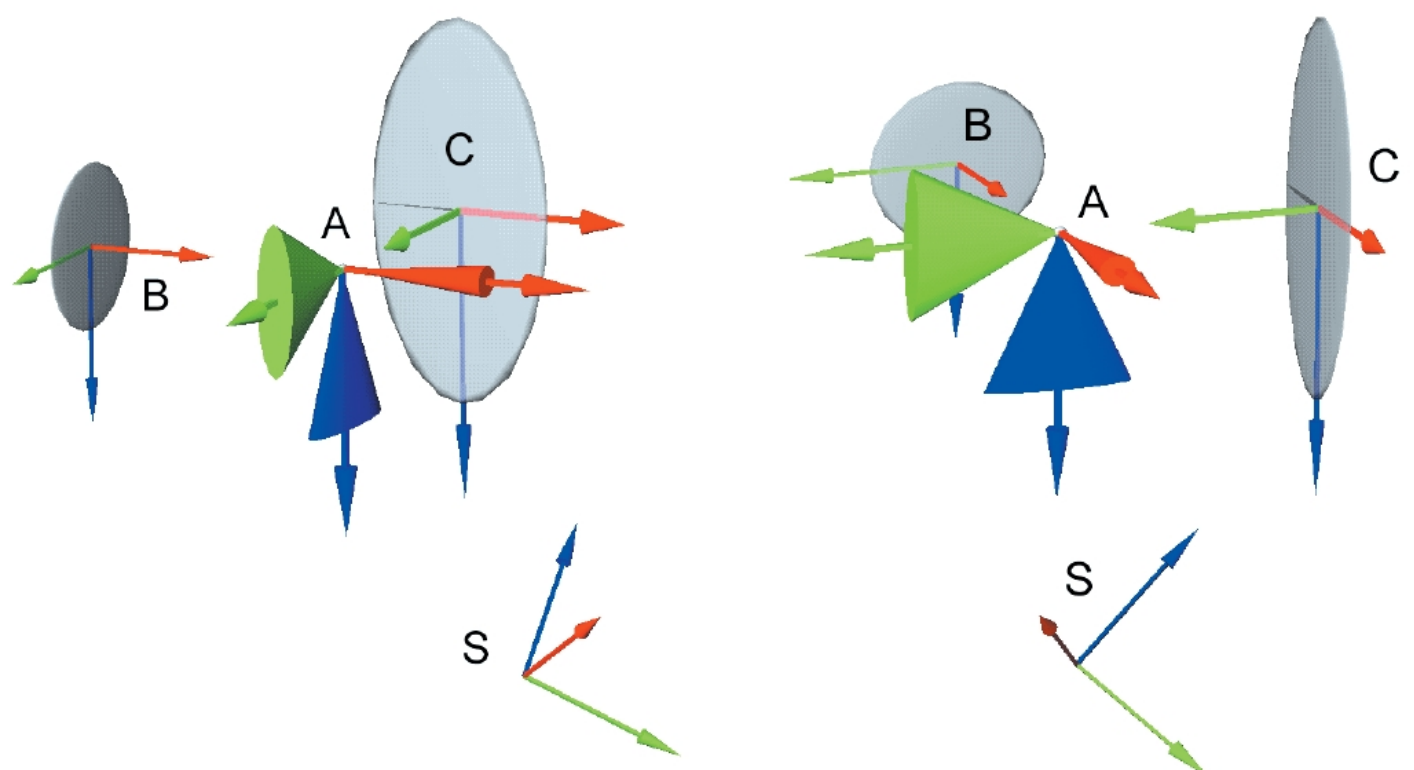

Figure 2.16: Two examples are shown of uncertainty in the pose of a coordinate system $\mathrm{A}, \mathrm{B}$, and $\mathrm{C}$ with respect to a sensor $\mathrm{S}$. Coordinate frame A shows uncertainty in the orientation of the pose rendered as cones about each axis. Translational uncertainty is depicted as ellipses in coordinate frames B and C [33]. [@2000 IEEE]

pay close attention to Bertin's specific rules for applying these variables such as adjusting the density of information in the graphic; too many or two few glyphs can make the visualization unreadable. Similarly, Tufte's data-ink maximization principle suggests that one wants to show the maximum amount of information while minimizing ink. Tufte's idea of representing numbers proportional to the magnitude of the quantity is clearly demonstrated by glyphs. The Gestalt laws of perception of spatial proximity, similarity, and symmetry described by Ware [112] are also applicable. The primary disadvantage of glyphs is that they may obscure important pieces of information or highlight information at the cost of blurring other data. 

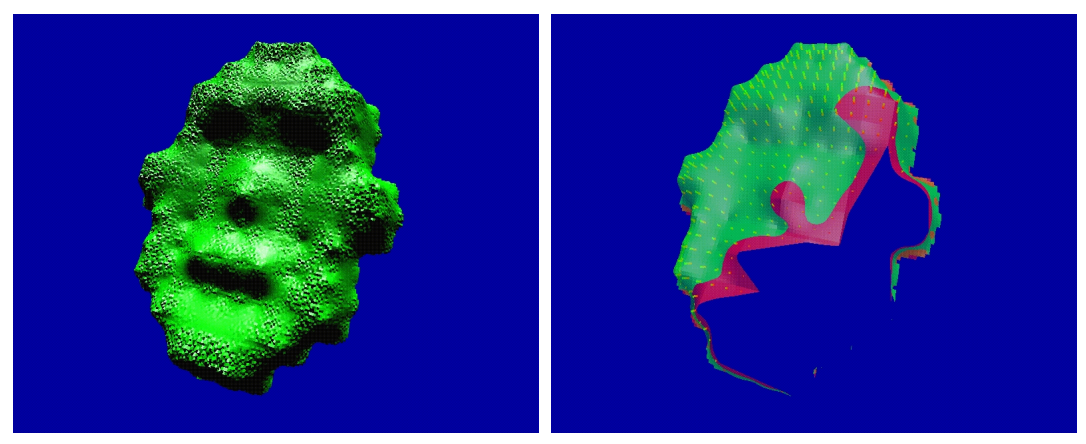

Figure 2.17: (Left) Bumpy surface created by surface displacement. (Right) Cross section of a fat surface [85]. [C1997 Springer]

\section{Geometry Addition/Modification}

Techniques that visualize error bounds typically modify the geometry of the surface based on the level of uncertainty. Examples in this category are fat surfaces [85], displacements and perturbations of geometry [85], and probabilistic surfaces [31]. Fat surfaces rendered the range of possible values in the data (see Figure 2.17). Probabilistic surfaces rendered the surface as a point set and displace each point along the surface normal by an amount proportional to the uncertainty (see Figure 2.18). Figure 2.19 conveys probability distribution of satellite image data [41].

The greatest impediment to successfully representing uncertainty by adding or modifying geometry can be described in terms of Tufte's data integrity principle. The principle states that data should not be shown out of context [105]. Indeed, these methods fundamentally show data out of context when they modify geometry by a statistical measure unrelated to the surface. More specifically, these visualizations are useful for representing positional uncertainty on the surface of a 3D model (see, for example, Figure 2.17) where the displacement tells us something about the uncertainties in the surface. According to Bertin's theories [6, 120], it is easier for 

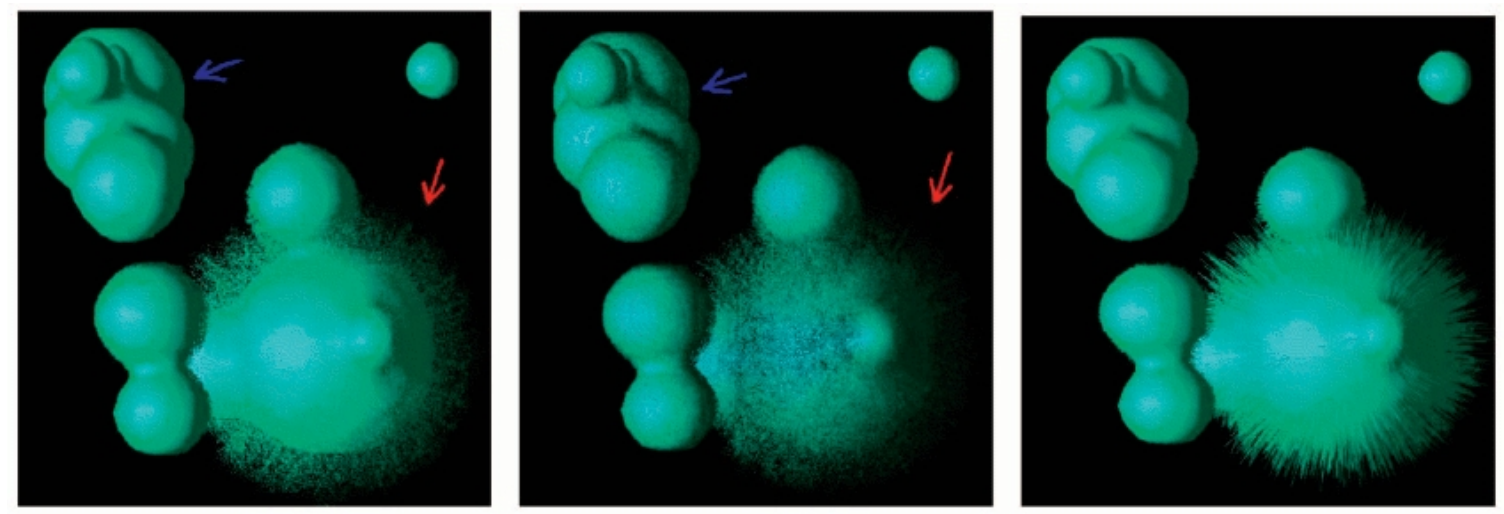

Figure 2.18: A probabilistic surface. (left) Opaque points, (middle) transparent points, and (right) opaque lines are displaced along the surface normals according the measure of uncertainty [31]. [C2004 IEEE]
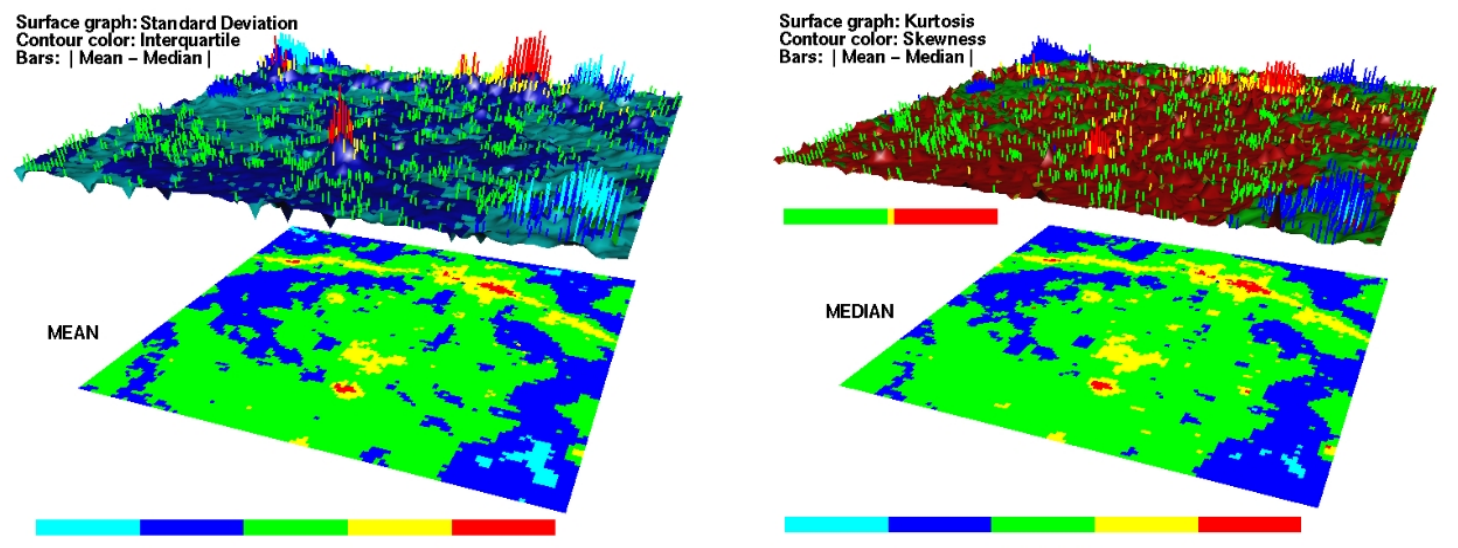

Figure 2.19: Visualization of satellite image data. (Left, top) Deformed by standard deviation and colored by interquartile range, height of the bars indicates absolute difference between mean and median. (Left, bottom) Image plane is colored according to mean. (Right, top) Deformed by the kurtosis field and colored by skewness, height of bars indicates absolute difference between mean and median. (Right, bottom) Image plane is colored according to median [41]. [C2001 IEEE] 

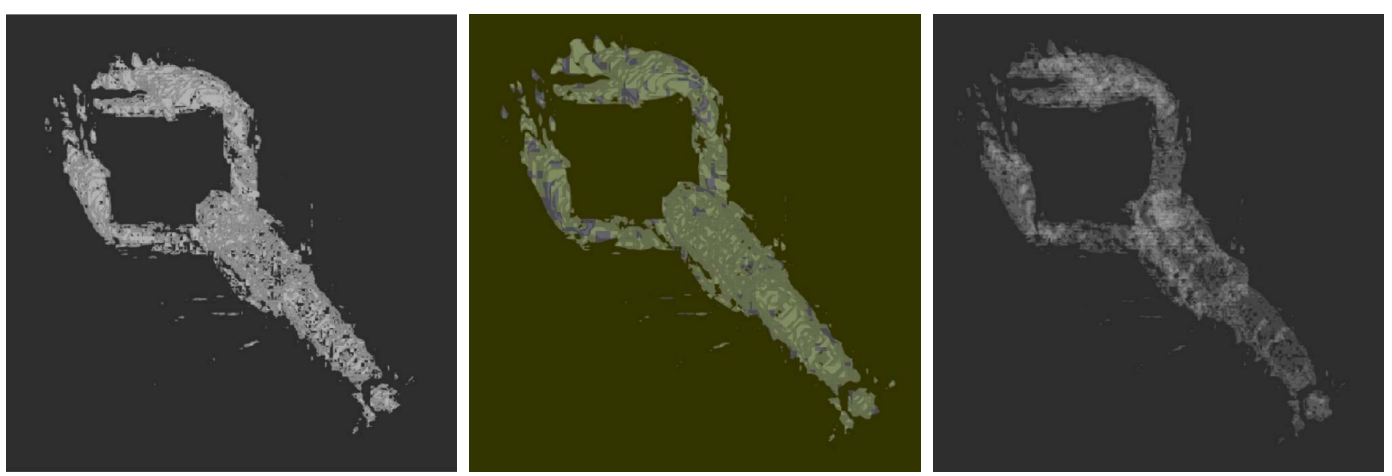

Figure 2.20: Example attributes representing uncertainty: (a) aliasing, (b) color, and (c) transparency. The change in aliasing, color, and transparency indicated magnitude of uncertainty [77]. [C2004 Elsevier]

us to cognitively integrate the visual variables (color, texture, and so on) than the added 3D surface. For example, in Figure 2.19 it is difficult to integrate the meaning of the statistical information as applied to the surface.

\section{Attribute Modification}

One of the most common methods of incorporating uncertainty into a visualization is by modifying attributes such as transparency, shading, opacity, texture, color, and aliasing of the geometry. In Figure 2.20, areas of high uncertainty are rendered with aliasing, color, and transparency [77]. Figure 2.21 applies texture to uncertainty [10]. Hue and opacity are varied in Figure 2.22 [91]. Transparency is used to convey uncertainty in [90].

Modifying attributes is a classic example of Bertin's [6] ideas of manipulating visual variables. Bertin gives specific examples of how these variables should be adjusted for optimizing their effect. Adjusting opacity, transparency, texture, and aliasing has 

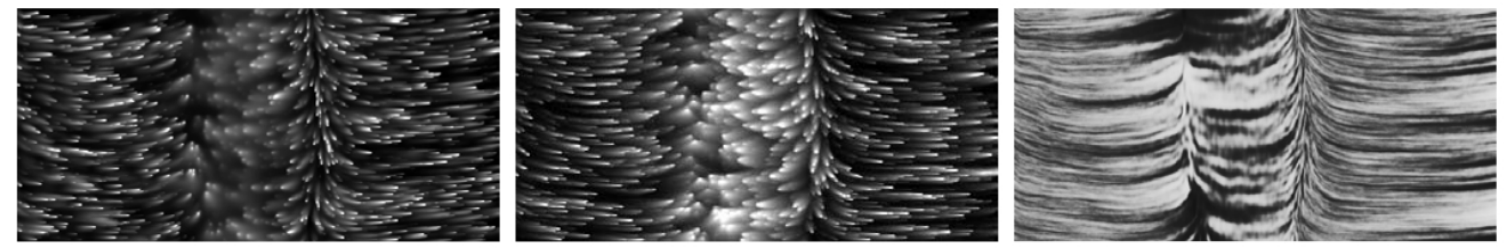

Figure 2.21: Three textures representing uncertainty in flow fields [10]. [C2005 IEEE]
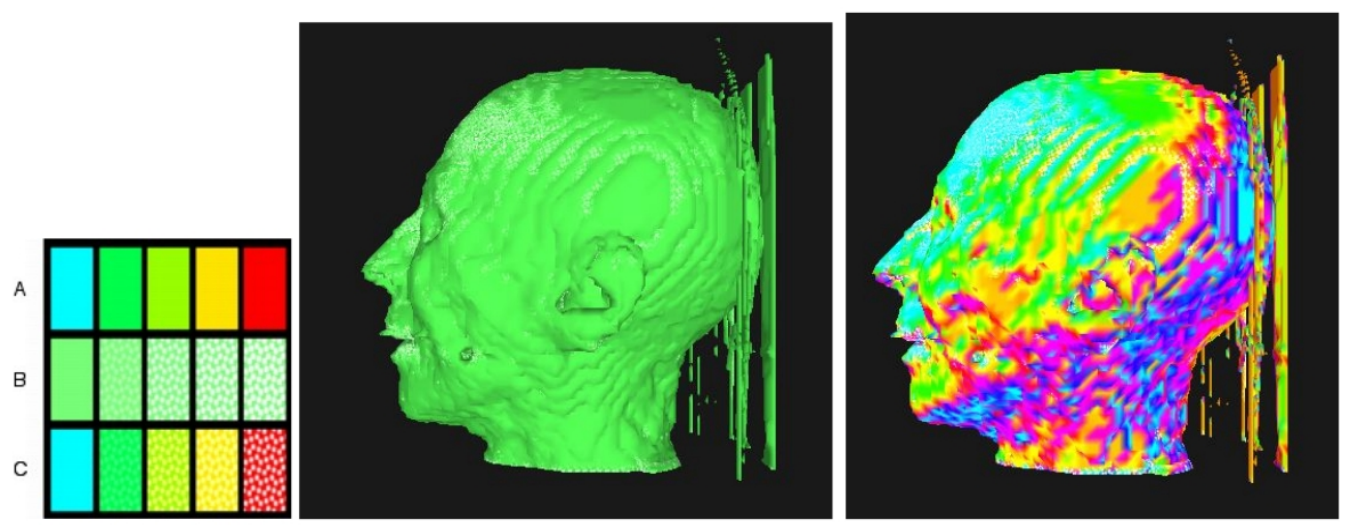

Figure 2.22: (Left) Shows variations over time in hue, texture, and texture and hue combined. (Middle) Uncertainty mapped to texture over time. (Right) Uncertainty mapped to opacity and hue [91]. [C2003 IEEE]

the practical advantage of not greatly increasing rendering time. For example, Djur-

cilov et al. [16] incorporated uncertainty directly into volume rendering by adjusting the transfer function according to the amount of uncertainty. Blurring the uncertainty had the effect of highlighting particular objects. As Ware [112] described, our visual system distinguishes between foreground and background in a similar way.

\section{Animation}

Animations use, for example, the speed of flicker to indicate the level of uncertainty [76]. Figure 2.23 shows two frames of an animation in which color oscillates 

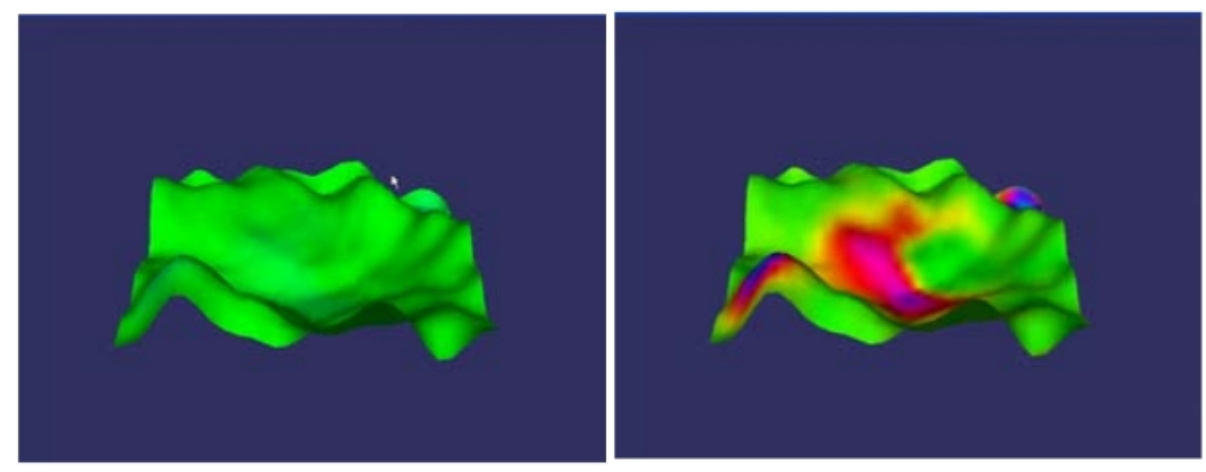

Figure 2.23: Two frames extracted from an uncertainty animation. Hue oscillates from the first frame to the second, corresponding to uncertainty [12]. [@2004 ACM]

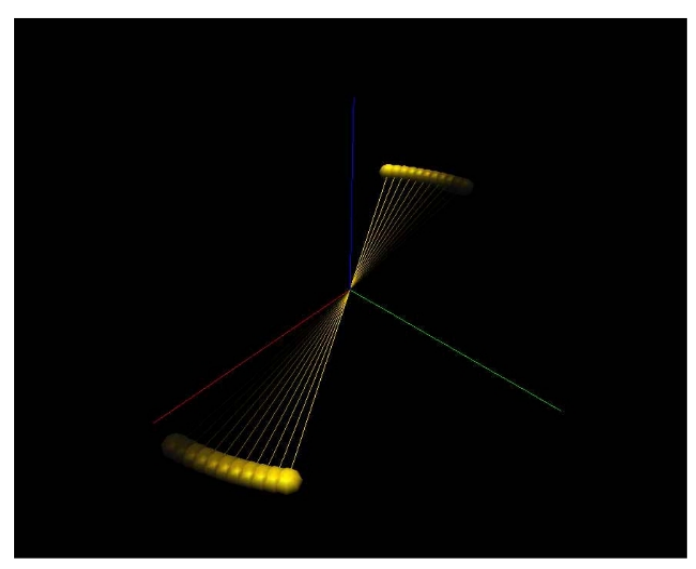

Figure 2.24: An animated uncertainty "twirling baton" glyph. The glyph changes in size and color [56]. [C1996 IEEE]

from the first frame to the second according to the uncertainty [12]. In Figure 2.24, a glyph changes size and color depending on the magnitude of uncertainty [55].

Several criticisms can be made of uncertainty animations. Oscillating green and red colors is not suited to the $10 \%$ of the male population and $1 \%$ of the female population that have some sort of color blindness [112]. For most applications, animating uncertainty adds another dimension to the data which contradicts principles devised 
by Tufte [105]. Moreover, rendering the animation in real time is not always possible. For example, in computer-assisted surgery, one could show positional uncertainty in the tracking system by animating the surface of a 3D patient model. This is impossible with current processor speeds, because the uncertainty must be updated as the tracking information changes many times each second.

Animation would be an ideal method for signaling that the magnitude of uncertainty has become too great. Ware [112] describes the role that motion plays in attracting our attention. Our peripheral vision is color blind so motion is a good choice for drawing our attention. The speed of oscillations can be changed to support high levels of awareness (fast blinking) or low levels of awareness (slow blinking).

\section{Confidence Images}

Confidence images are images that convey statistical confidence information. The advantage of displaying confidence information is that confidence intervals are easily understood by practitioners in a variety of disciplines. Confidence information supports Tufte's [105] data-ink maximization principle because a great deal of information can be conveyed with the additional of a small amount of ink. A good example of the application of this principle is Figure 2.25. It shows a weather forecast for temperature and the forecast with added confidence intervals [76]. Note that the confidence interval does not obscure or detract attention from other components of the visualization.

In Figure 2.26, an uncertainty method that helps in the classification and grading

of brain tumors is shown [108]. The size of each square corresponds to the ratio of magnetic resonance spectra. The sizing of the boxes relates to Tufte's theory that 

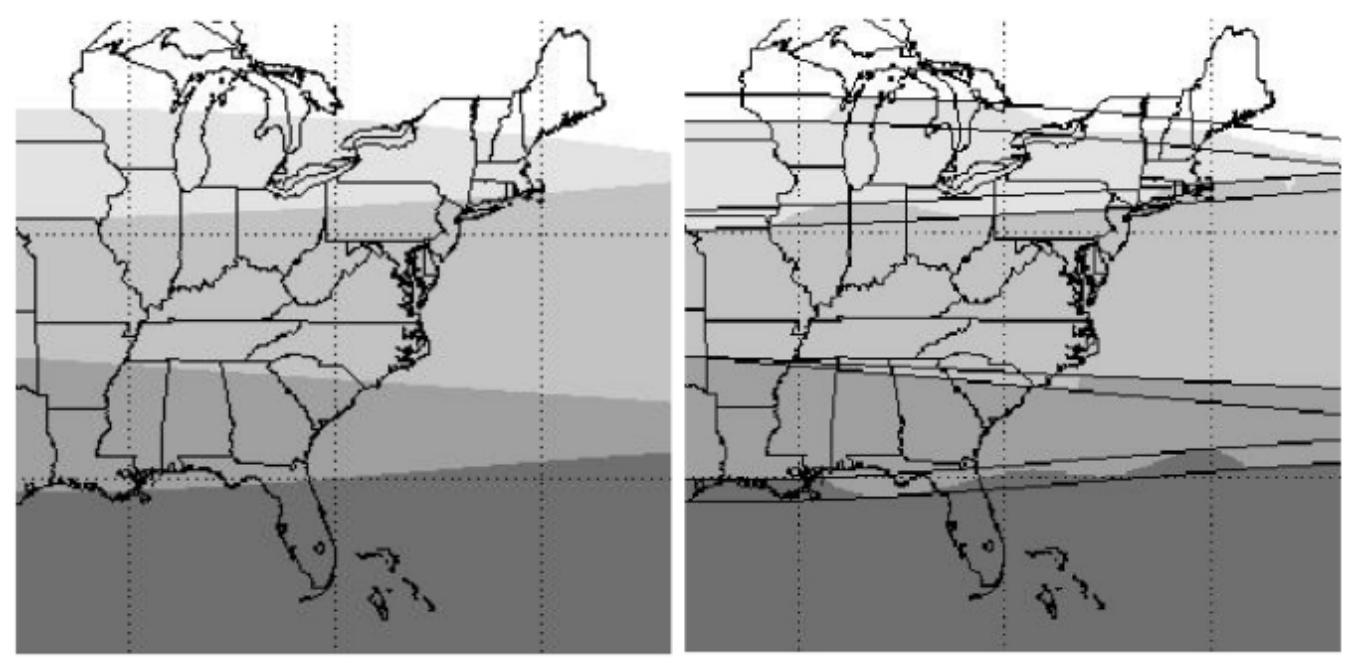

Figure 2.25: Weather forecast depicting (left) original forecast and (right) forecast with confidence values shown [76]. [C2000 Nagy and O'Leary]

the size of the graphic is proportional to the quantity represented. The grouping of the boxes makes sense perceptually because Gestalt laws state that similar objects are grouped together [112]. However, the boxes should be resized to comply with Tufte's [105] data density principle.

Figure 2.27 demonstrates boundary uncertainty in the position of anatomical structures using direct volume rendering of brain image data [46]. Figure 2.27, subfigure $\mathrm{C}$ renders confidence intervals onto the volume data. The challenge of rendering three dimensional data is that some data may be obscured when additional information is shown. Ware [112] suggests that visualizations should allow the user to vary the level of detail shown. This visualization is a good example of rendering different levels of detail. 


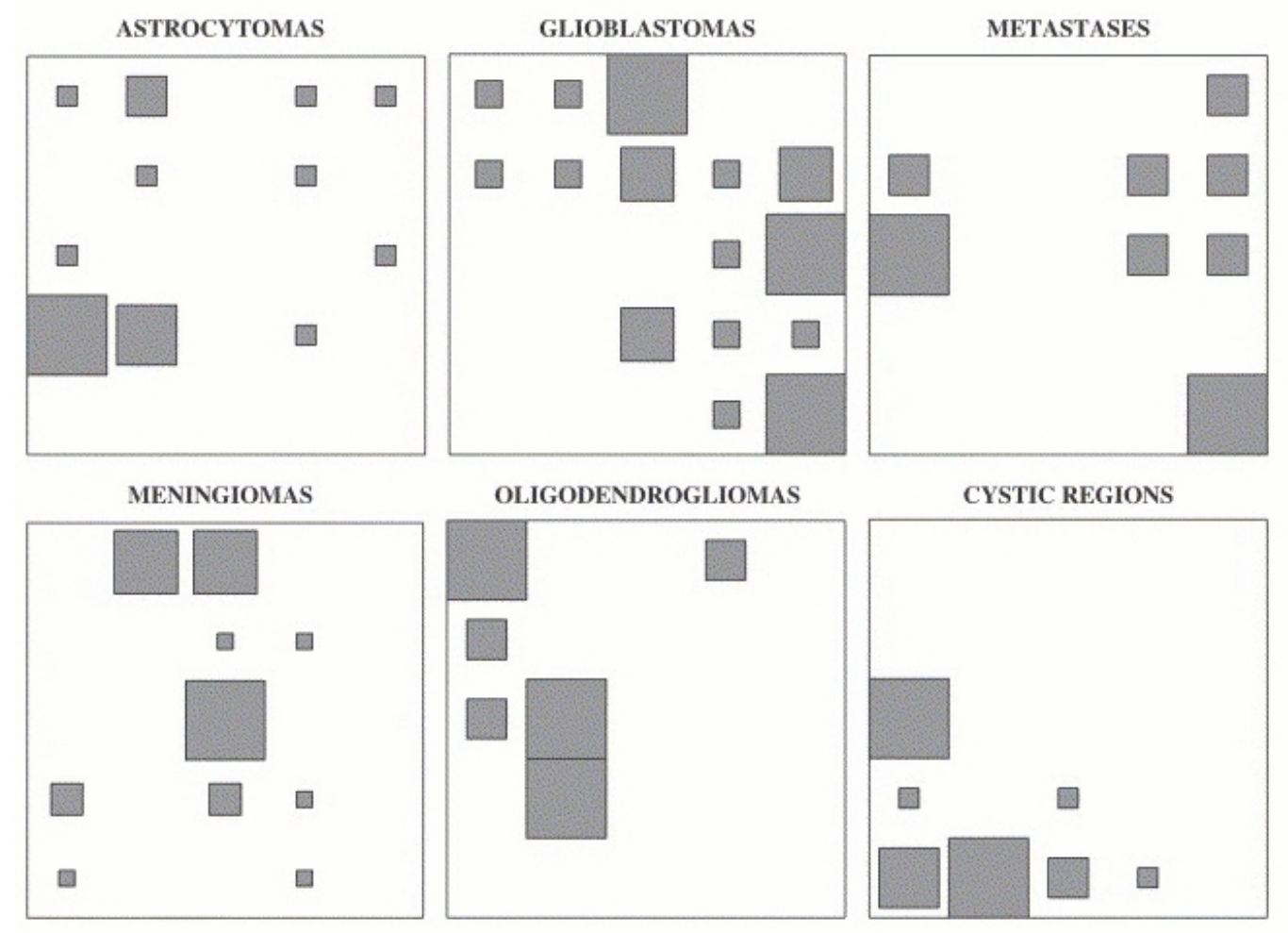

Figure 2.26: Representation of uncertainty in the five main tumor types using magnetic resonance spectra. The size of each square corresponds to the ratio of the level of spectra [108]. [C2006 Elsevier]

\section{Charts and Graphs}

The traditional method of graphically representing uncertainty was to add error bars to graphs. In the method shown in Figure 2.28, Olston and Mackinlay [84] proposed using ambiguation to show uncertain boundaries in charts. In McQueary et al. [70], a hyperbolic chart used to incorporate uncertainty information into decision making in battle. Figure 2.29 demonstrates how simple graphs can display uncertainty information for clinicians [68].

The advantage of using charts and graphs for representing uncertainty information 

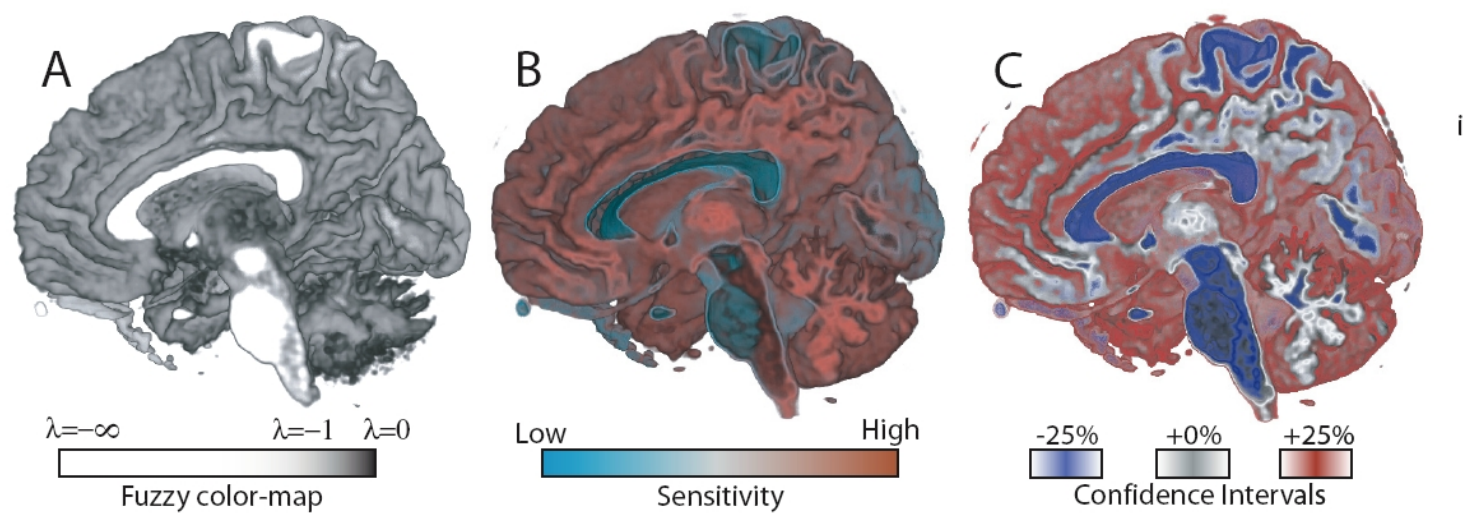

Figure 2.27: Direct volume rendering of brain image data. Subfigure A is a fuzzy volume rendering. Subfigure B shows sensitivity information. Subfigure C demonstrates confidence intervals for uncertainty in the position of structures due to volume rendering [46]. [C2005 IEEE]

is that they improve the data density of the graphic by adding additional information with minimal ink [105]. Using unusual visualization methods, like modifying geometry or using animation, lets us see unexpected features in the data which may not be apparent by simply charting the data.

\section{Conveying Uncertainty Information with Sound}

Another method of conveying uncertainty is that of using sound. Uncertainty can be conveyed using using frequency, stereo field, volume, rhythm, tempo, and timbre of sound [72]. Sonification can be used to convey information that can not be visualized or to complement an uncertainty visualization. An advantage of uncertainty sonification is that a visualization does not become cluttered; sonification gives another means of conveying information. LISTEN, a data sonification system, has been combined with visualizations of fluid flow and geometric uncertainty [56]. 


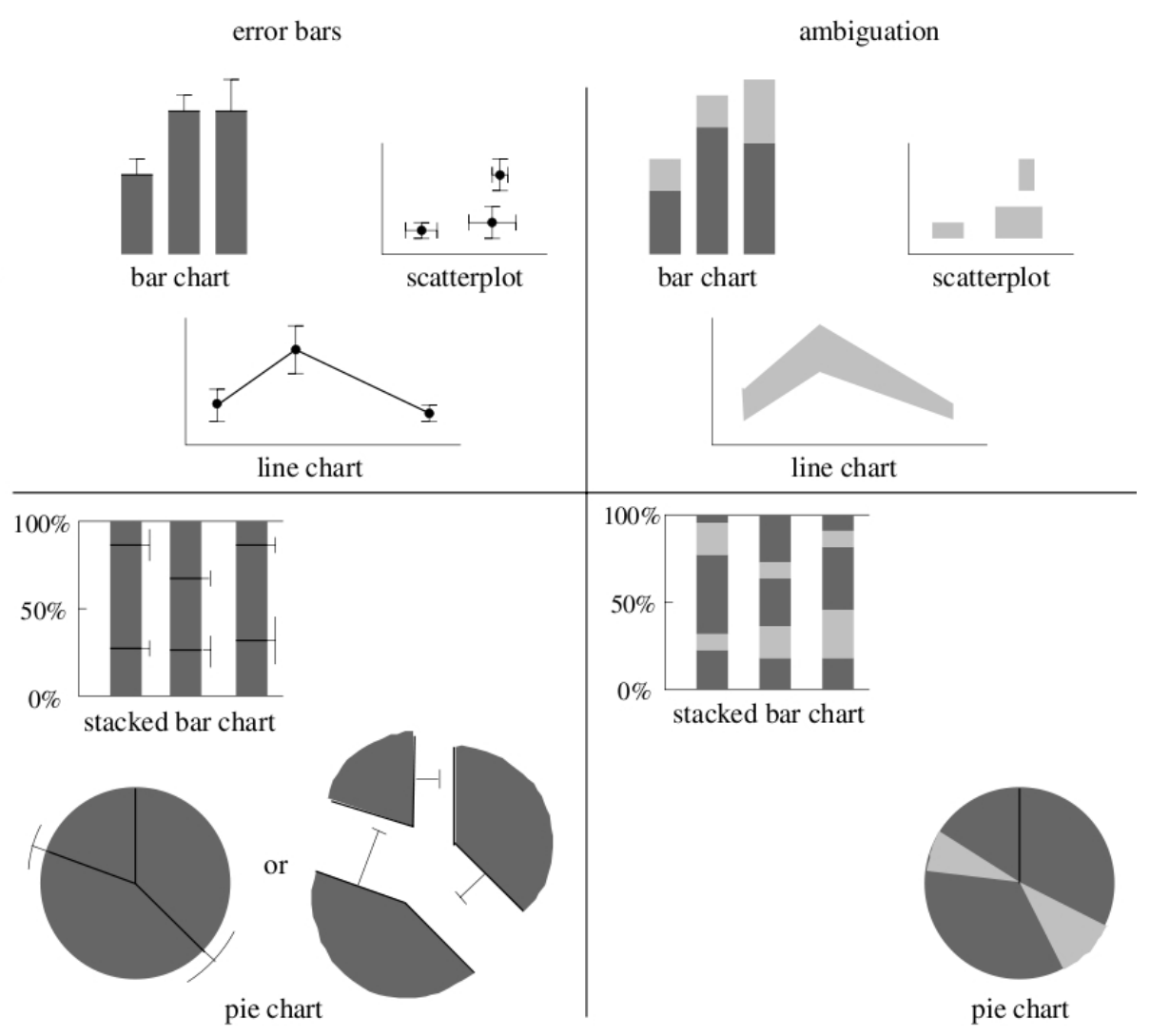

Figure 2.28: Common chart types shown with error bars (left) and added uncertainty information (right) that the authors call ambiguation. Ambiguation draws the region between possible boundaries as fuzzy [84]. [C2002 IEEE]

\subsection{Summary}

This dissertation contributes to the navigation paradigm in CAS in which preoperative images are used for guidance. Methods of computing and validating registration uncertainty have recently been reported in the literature. Tracking uncertainty has also been examined. However, these methods require a precision measurement device (Optotrak tracking system, coordinate measurement machine, or machined tool) to establish ground truth. Several interesting research questions remain open, such as: 


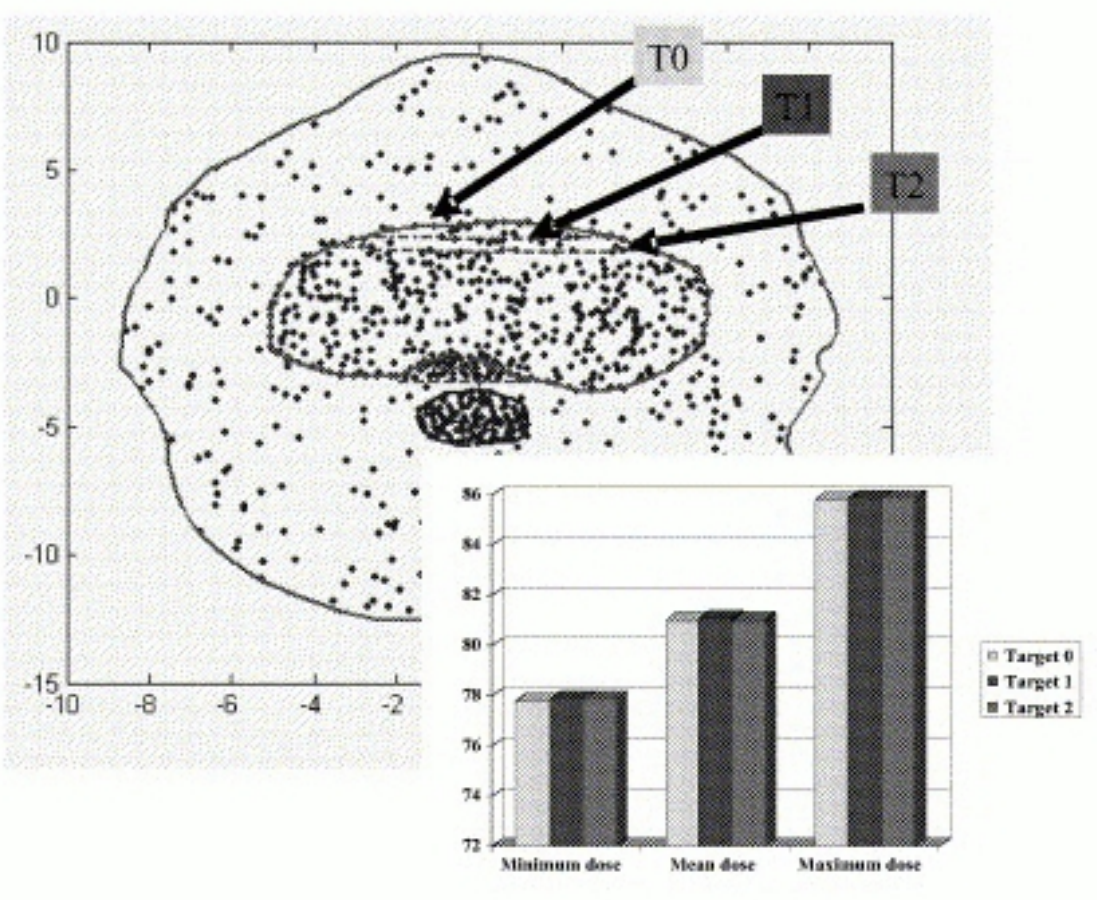

Figure 2.29: Graph represents maximum, average, and minimum doses of radiation required in regions within the indicated regions [68]. [C2004 Elsevier]

Can tracking uncertainty be computed in the absence of ground truth and can we show that this estimate is reasonable? How does the uncertainty from tracking propagate to registration and calibration? Can uncertainty be computed in real-time in the operating room? These questions are addressed in Chapter 4.

Considerable anecdotal and qualitative evidence exists on how to design effective visualizations $[105,112]$ and on effective uncertainty visualizations $[1,35,50,77]$ but to my best knowledge, no user studies have yet demonstrated the superiority of uncertainty visualization over methods that do not visualize uncertainty. Perhaps this is because it is difficult to define a task that is representative of a typical interaction with the visualization that can be statistically analyzed. No widely accepted 
uncertainty visualization methods exist for computer-assisted surgery. In Chapter 3, a preliminary study of uncertainty visualization is described. Chapter 5 describes an in-depth comparison of uncertainty visualizations in the form of a user study for computer-assisted surgery. 


\section{Chapter 3}

\section{Study: Does Registration Uncertainty Improve Surgical Navigation?}

In this chapter, I describe an examination of whether uncertainty visualization in computer-assisted surgery could improve the practice of surgery by helping surgeons make more informed decisions in the operating room in the presence of imperfect data. There are many problems in computer-assisted surgery that could benefit from uncertainty visualization.

Commercial surgical displays show guidance information to the surgeon as though it were perfect. But there is measurement uncertainty inherent in the proposed trajectory and in the tracked tool position, which can have serious consequences to the patient. Consider, for example, delicate spinal surgeries.

In this chapter, I describe a pilot study performed by my colleagues and me in which registration uncertainty was visualized [98]. I describe the method we used for uncertainty visualization when guiding a surgical instrument along a linear path to a point target. I performed a user study to quantify the effectiveness of the visualization technique. The results showed that the uncertainty visualization technique 
improved user performance. We also attempted to measure how subjects interpreted the visualization. The main results of this chapter are:

- Subjects required fewer attempts at locating a tumor when uncertainty was visualized.

- Subjects failed to locate a tumor less often when uncertainty was visualized.

- Subjects used the uncertainty visualization to guide their search for the tumor.

- There was strong evidence that visualizing uncertainty in the operating room could improve surgical efficacy.

To the best of my knowledge, no user studies exist that examine direct user interaction with an uncertainty visualization.

\subsection{Registration Error}

The rigid registration transformation has six degrees of freedom (three translational and three rotational parameters). One way to report registration error is to visualize the uncertainties in all six degrees of freedom (see Figure 3.1). Such visualizations convey important information (for example, the rotational component about the $z$ axis is bimodal and much broader than the other two rotational components). But, such a visualization lacks spatial intuition.

\subsubsection{Registration Uncertainty}

Recently, our research group proposed a sampling method for computing uncertainty in registration $[59,60]$. Retrospectively, the uncertainty of the registration parameters was computed using a particle filter as part of the registration algorithm. This 

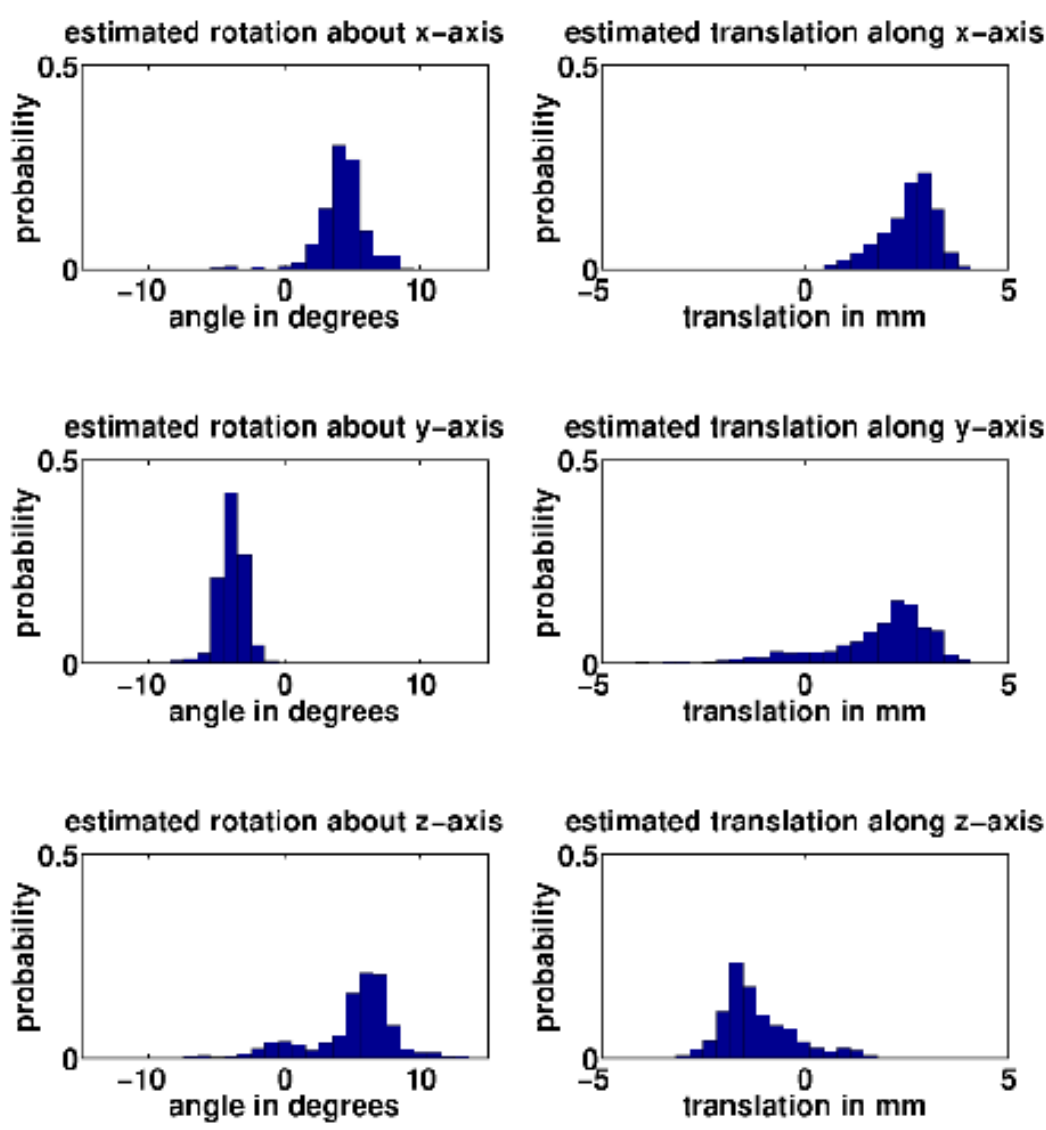

Figure 3.1: Estimated distributions of the six parameters for rigid registration of the patient in the coordinate frame of the tracking camera.

algorithm produced a sampled distribution of the registration parameters rather than a single estimate of the parameters.

Note that the visualization of these distributions (Figure 3.1) lacks intuition and meaning to those unfamiliar with registration error. Moreover, existing techniques for uncertainty visualization do not apply to our problem. Sampling techniques consider uncertainty at a single point in space; I wanted, however, to visualize the entire distribution of error. Moreover, I was interested in visualizing the distribution of 
error in the instrument orientation and position, rather than on the surface of the anatomy, because it is the instrument that the surgeon attempts to place.

\subsection{Osteoid Osteoma Excision}

This study of uncertainty visualization was based on osteoid osteoma excision. An osteoid osteoma is a small, painful, benign bone lesion that occurs most frequently in the long bones of teenagers and young adults (more common in males than females). In the United States, osteoid osteomas account for $12.1 \%$ of benign tumors and $2.9 \%$ of all tumors [13]. These tumors are very painful. In some patients, the tumors resolve on their own over time for unknown reasons. In other cases, successful treatment requires complete removal of the nidus surgically. My research group used a minimally invasive technique to remove the tumor using a tracked needle to expose the nidus [21].

The primary surgical task of contacting a tumor in an osteoid osteoma excision is similar to other tasks in computer-aided surgery, such as inserting radiation-emitting beads or positioning a screw for fixation plate placement.

\subsection{Methodology}

This section describes computing the effect of registration uncertainty on a linear path of the surgical tool, visualizing the resulting uncertainty distribution, and utilizing the visualization in a user study that mimics tumor excision.

\subsubsection{Registration Uncertainty Source}

The distribution of the registration parameters is approximately symmetric, anisotropic, and leptokurtic (strongly peaked) with heavy tails $[59,60]$. In this study, 
my colleagues and I investigated the distributions of the registration parameters and the resulting effects on a planned linear path. We built an isosurface model of the proximal end of a cadaver femur, and identified a linear path between the lateral cortex of the femur and the medial inferior cortex of the femoral neck such as might be followed in an osteoid osteoma excision (Figure 3.2). A rapid prototyping machine was used to produce a plastic model of the isosurface model. A calibrated stylus and a Polaris tracking system (Northern Digital Inc., Waterloo, Canada) were used to digitize five distinct landmarks and an additional 50 points from the entire surface of the plastic model; these 55 points were used to compute a good estimate of the ground truth registration transformation $\mathbf{T}_{0}$. To generate registration point sets for learning the registration parameter distributions, we collected 10 registration points from each of 16 separate regions near the lateral entry point. We generated sets each with 16 points by randomly drawing one point from each of the 16 groups of points; 10,000 sets were generated and registered to the surface model using ICP [7] to produce a set of registration transformations $\left\{\mathbf{T}_{i} \mid i=1 \ldots 10,000\right\}$. A computation of the 10,000 target registration errors (TREs) using the virtual tumor location as the target produced a similar distribution to that observed by Ma and Ellis [60] (Figure 3.2).

\subsubsection{Visualization Method}

We applied each of the difference transformations $\boldsymbol{\Delta}_{i}=\mathbf{T}_{i} \mathbf{T}_{0}^{-1}$ to the planned path to produce the empirical distribution of paths under registration uncertainty. The simplest visualization technique is to render the paths as individual lines. However, this does not produce an adequate representation of the spatial distribution of the 

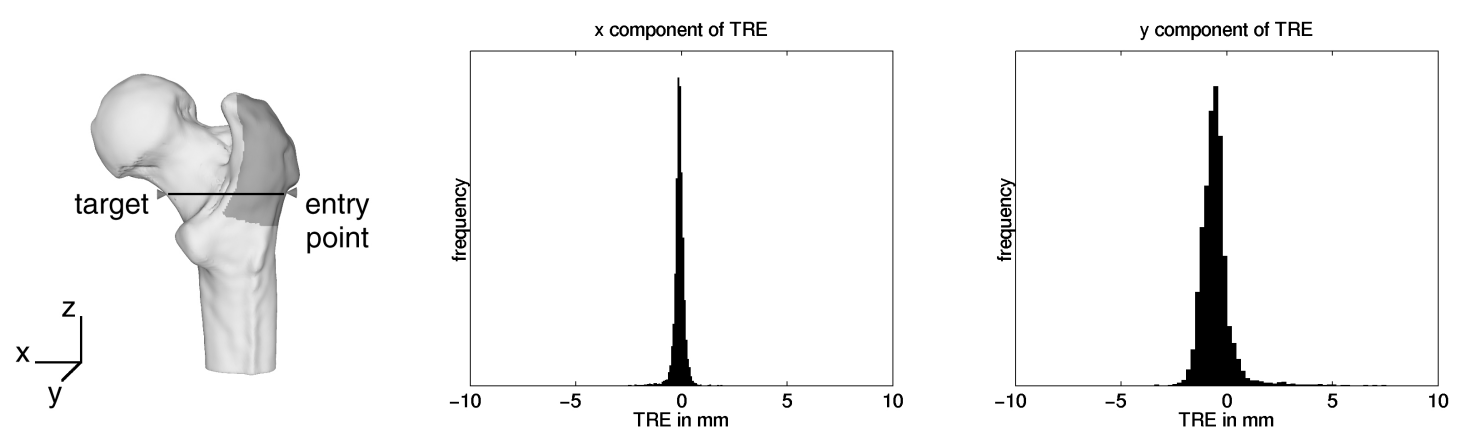

Figure 3.2: (Left) Model of the proximal femur showing the registration region in gray and the planned path between the entry point on the lateral cortex and the target point on the medial inferior neck. (Middle and right) The TRE error distribution of the target point under the 10,000 training registrations; the $z$ component of TRE was similar to the $x$ component.

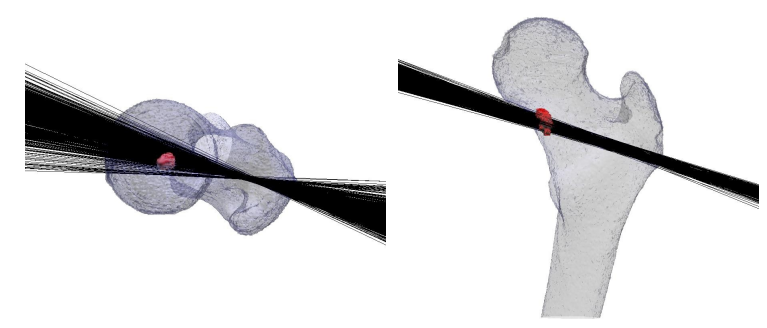

Figure 3.3: An example of a tumor (red) on the femur rendered with the distribution of possible tool trajectories (black lines) in the front view (right) and top view (left).

paths (see Figure 3.3). To convey information about the spatial distribution of the paths, we chose to use volume rendering. With volume rendering, we could view the path distribution as a 3D volume or as 2D cross sections, and we could choose what features to accentuate or attenuate by modifying the color and opacity transfer functions.

Our path distribution volume had its long axis aligned with the mean path direction. We computed each slice of the volume at $1 \mathrm{~mm}$ intervals (with slices oriented perpendicular to the long axis) by computing the intersection of every path with the slice. Given that the TRE distribution was approximately symmetric with 

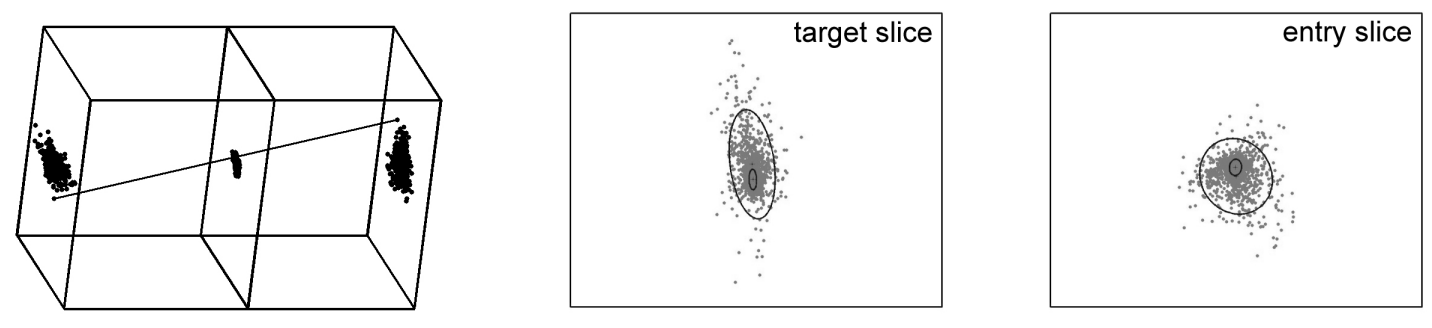

Figure 3.4: Illustration of the path distribution volume. (Left) The planned path under each difference transformation $\boldsymbol{\Delta}_{i}$ is intersected with each slice of the volume, and a 2-component mixture of the Gaussians is fit to the intersection points. (Middle and right) Path intersection locations at the target slice (middle) and entry slice (right). The ellipses are centered at the means of the Gaussians; their size and orientation reflect the structure of the covariance matrices. Note the anisotropic distribution at the target location.

a significant central spike and broad tails, we fitted a two component mixture of Gaussians to the intersection points on each slice; one Gaussian distribution represented the central spike and the other represented the tails. We used a greedy EM (Expectation-Maximization) algorithm to fit the mixture of Gaussians [110].

Figure 3.4 illustrates the volume construction method. All of the volume visualizations were performed with the Visualization Toolkit (Kitware Inc., New York, USA) using texture-based volume rendering [22]. Figure 3.5 shows the volume rendered path distribution from two different views. Figure 3.6 shows an apparent twisting of the path distribution made apparent by tuning the opacity transfer function. Figure 3.7 shows the volume and its relationship to the femoral model.

\subsubsection{User Study}

The study mimicked the excision of a deep bone tumor. In this task, the surgeon must expose a blind target (tumor) by drilling through the bone and is immediately aware of success as the tumor is readily visible through the drilled hole. We quantified the 


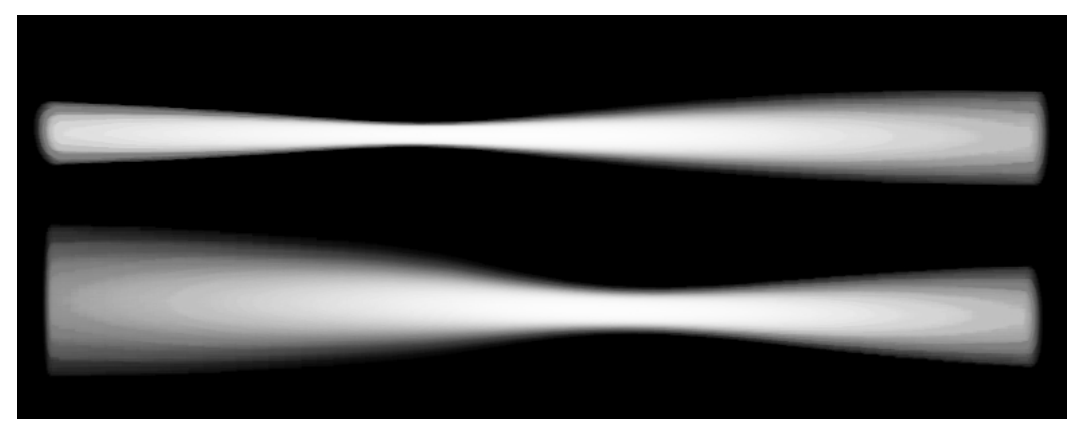

Figure 3.5: The volume rendered path distribution from (top) the frontal view and (bottom) the top-down view.

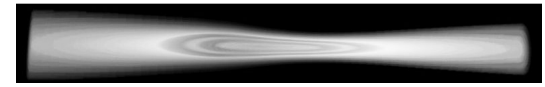

top view

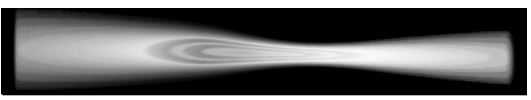

side view

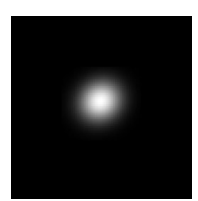

axial view

Figure 3.6: Path distribution rendered as a volume.

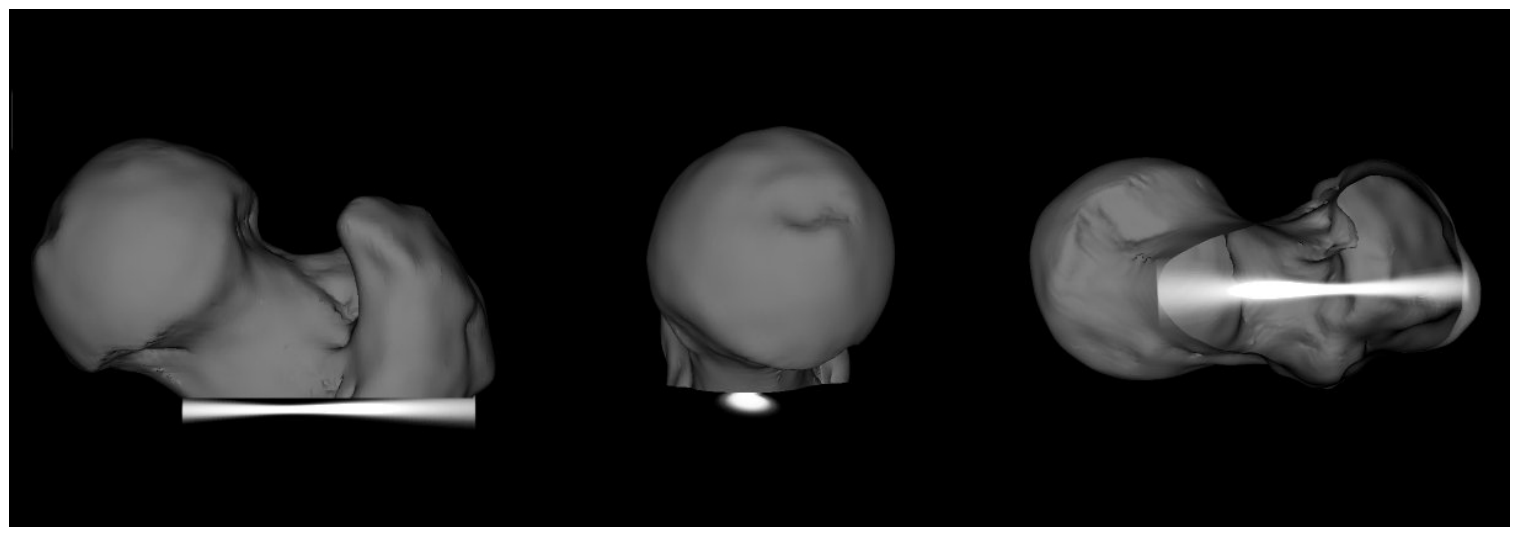

Figure 3.7: Model of the proximal femur showing the uncertainty visualization of the planned path between the lateral cortex and the target point (location of tumor) on the medial inferior neck. 
success of this task by measuring the number of attempts required to hit the target (tumor).

\subsubsection{Subjects}

Thirty non-expert subjects (9 female, 21 male, 28 right-handed, 2 left-handed) from the Queen's University community participated in the study. The experiment took approximately ninety minutes for each subject to complete. Subjects were supervised in all tasks.

Ethics approval for the experiment was obtained from the Queen's University General Research Ethics Board. All subjects signed a waiver prior to participation in the study (see Appendix C).

\subsubsection{Apparatus}

We used a Polaris optical tracking system (Northern Digital Inc., Waterloo, ON, Canada) to track the position and orientation of a calibrated surgical tool and metal targets. Dynamic reference bodies (DRBs) were attached to the tool and targets so that the camera could record their positions. Ten metal targets, each $5 \mathrm{~mm}$ in diameter, were mounted to an optical bench in an irregular arrangement. The targets were wired to a buzzer that sounded when the surgical tool touched the target. Modeling clay obscured the targets from view. An eighteen inch computer monitor placed in the line of sight of the subject (approximately one meter in front) displayed guidance information to the subject during the experiment (see Figure 3.8). 


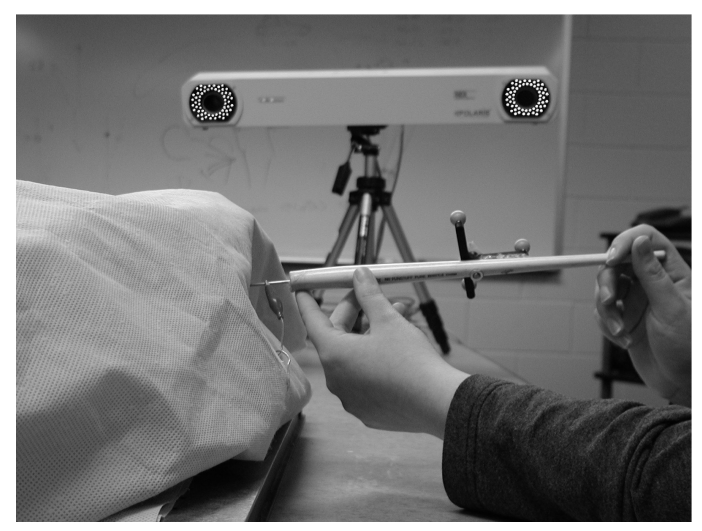

Figure 3.8: Illustration of apparatus used study. A Polaris tracking system tracked the position and orientation of DRBs located on the surgical tool and targets. The metal targets were mounted to a board in an irregular array and obscured by clay. A buzzer indicated when a target was touched by the surgical tool.

\subsubsection{Stimuli}

We performed another 1,000 registrations in a similar way as described in Section 3.3.1 using a different set of candidate registration points. We randomly selected 10 registrations that produced a TRE of greater than $4 \mathrm{~mm}$. These uncertainty distributions would guarantee that the subjects would miss on the first attempt if they followed the path of highest probability perfectly. Furthermore, the uncertainty distributions were chosen such that subjects were not guaranteed to find the target within three standard deviations of the center of the uncertainty distribution, as this would bias the results in favor of the uncertainty visualization technique. This is because we could simply choose registrations which would guarantee that the subject would always find the target within the uncertainty visualization. These registration transformations were transferred to the array of targets by aligning the planned path on the femoral model to each target.

A user interface was developed that showed three orthogonal views of the planned 
trajectory and the current tool position. A line and circle represented the planned path of the tool and location of the target. The planned path and target were overlayed with the uncertainty visualization. Subjects were asked to insert the surgical tool to touch a target obscured by modeling clay using the user interface for guidance. If the subject failed to touch the target, the tool was withdrawn and the subject was asked to attempt the task again. Red circles indicated unsuccessful attempts on the screen. If the subject touched the target, a buzzer sounded. The subject was allowed ten attempts to touch the target; if the target was not found, the task was considered incomplete.

The number of attempts that the subject required to find the target and the position of the tip of the tool for each attempt were recorded using an optical tracking system.

\subsubsection{Design}

We used a within-subjects design for our user study. First, subjects were given a verbal description of how to navigate the three orthogonal views and given the opportunity to practice with the views. Second, the visualizations were explained to subjects. In the task without uncertainty visualization, subjects were told to locate the target using whatever system made sense to them. In the task with uncertainty visualization, subjects were told that the ellipse rendered around the target corresponded to approximately the $95 \%$ confidence interval, which meant that the greatest uncertainty was in the largest axis of the ellipse and that, most likely, the target lay in that direction. Each subject was trained with a set of five preliminary trials of each visualization type. Subjects were presented with ten tasks with the uncertainty 

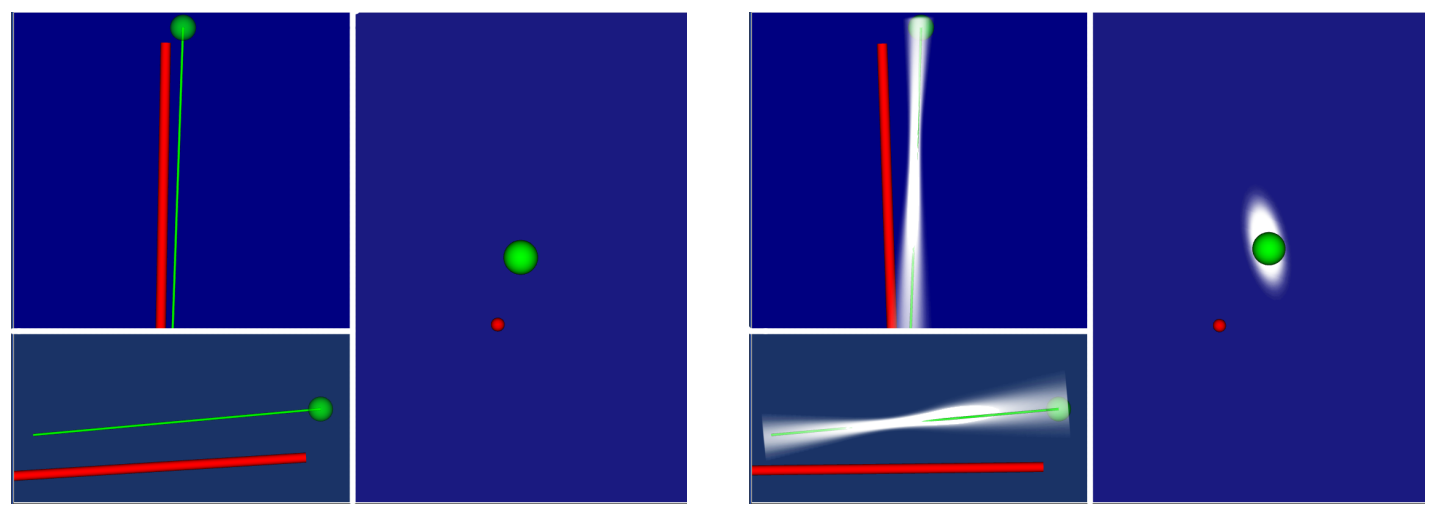

Figure 3.9: (Left) User interface with no uncertainty visualization. This is a typical visualization given to surgeons in computer-assisted surgery. The three orthogonal views are (clockwise from right) axial, side, and top. The planned path of the surgical tool is the green line. The target location is the green sphere. The current, tracked path of the tool is the red cylinder and red dot. (Right) User interface with uncertainty visualization. An uncertainty distribution has been rendered around the planned path and target. If the tool is guided correctly according to the plan, but the target is not reached, the planned path must have been incorrect due to registration error. In this case, the surgeon can try alternate paths according to the uncertainty distribution. Note that all of the subjects could distinguish between red and green colors.

visualization and the same ten tasks without the uncertainty visualization (see Figure 3.9). The order of the tasks was randomized within each set and the order in which subjects were given the types of visualizations was interchanged in order to reduce the effects of learning. Subjects one through fifteen were shown of set of planned paths and targets without uncertainty visualized followed by a set with uncertainty visualized. Subjects sixteen through thirty were shown the visualization types in the opposite order.

In order to study the subject's probing pattern, we chose uncertainty distributions that would guarantee that the subjects would miss on the first attempt if they followed the path of highest probability perfectly. Furthermore, the uncertainty distributions 
were chosen such that subjects were not guaranteed to find the target within three standard deviations of the center of the uncertainty distribution, as this would bias the results in favor of the uncertainty visualization technique.

The intuition behind the visualization is this: Subjects would try the area of high probability first and would likely fail because we chose distributions that would guarantee this. In subsequent attempts, their search would be guided by the low probability portions of the distribution. We expected that subjects would attempt to cover as much area of the distribution as possible starting in the high-probability areas.

\subsection{Results}

The hypotheses that were tested are:

Hypothesis 1 The number of attempts required by subjects is fewer when uncertainty is visualized than when uncertainty is not visualized.

Hypothesis 2 The number of incomplete tasks is reduced when uncertainty is visualized than when it is not visualized.

Hypothesis 3 The subjects cover a greater fraction of the target probability distribution when uncertainty is shown than when it is not.

Hypothesis 4 The distribution of subjects' attempts matches the actual uncertainty distribution when uncertainty is shown, and does not match when it is not shown.

I emphasize that we could have designed this experiment so that maneuvering the probe was trivial. We could have simply had the subjects pin-point exposed targets 


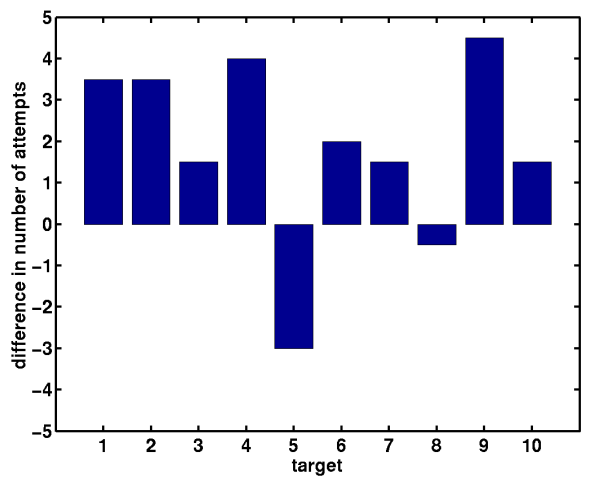

Figure 3.10: Difference between number of attempts without uncertainty and number of attempts with uncertainty. Values greater than zero indicate worse performance without uncertainty visualization and values less than zero indicate worse performance with uncertainty visualization.

without any kind of computer guidance, thus removing the difficulties associated with probe placement. We chose to mimic (as closely as reasonably possible) the actual conditions and data present in the operating room.

\subsubsection{Hypothesis 1: Fewer attempts with uncertainty}

A Mann-Whitney ${ }^{1}$ test showed that the number of attempts was reduced significantly when uncertainty was visualized $Z=-2.99(p<0.01)$. The mean number of attempts without uncertainty visualized was $66.1 \pm 2.2 \mathrm{SE}$ and with uncertainty visualization was $56.2 \pm 1.8 \mathrm{SE}$.

Figure 3.10 shows the difference between the number of attempts with and without uncertainty visualized. These results are strong evidence that subjects performed better when uncertainty is visualized.

\footnotetext{
${ }^{1}$ In this case, the Mann-Whitney test is more appropriate than its parametric analog, the $t$ test, for two independent samples, because the $t$ test assumes a normal distribution [95].
} 


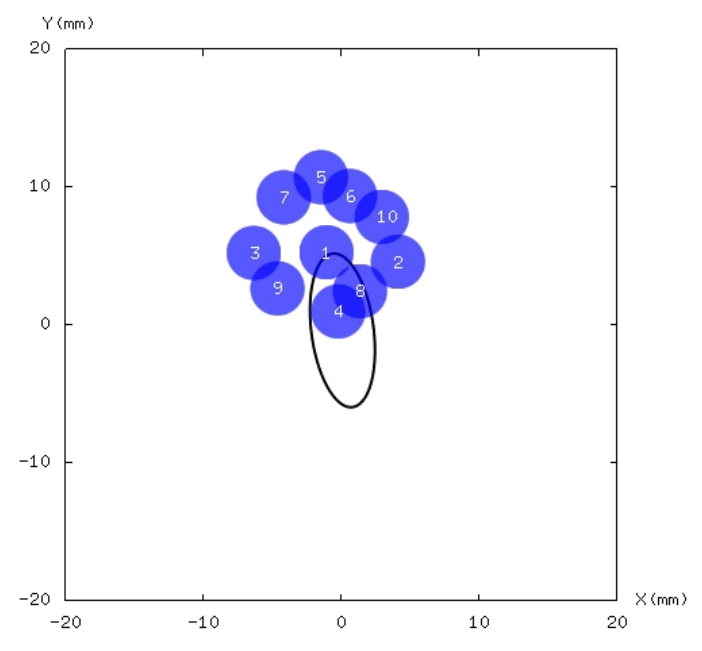

(a)

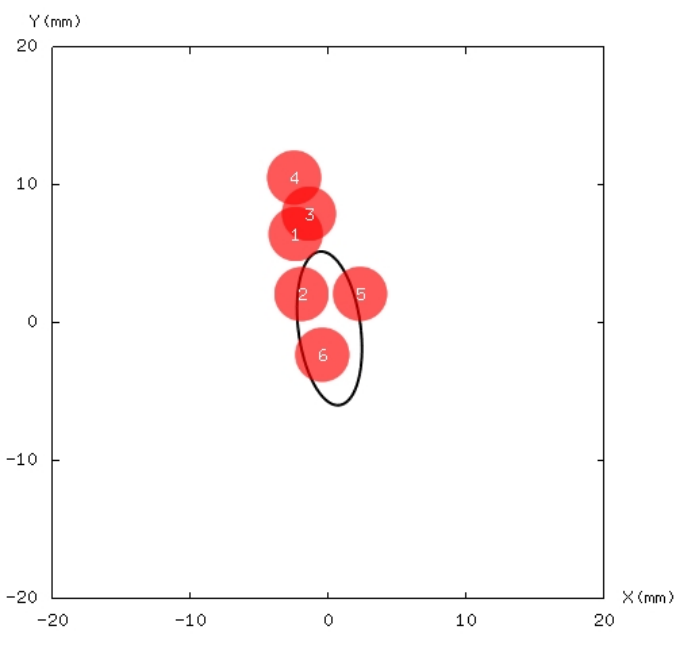

(b)

Figure 3.11: (a) An example of Subject A's probes when uncertainty was not shown. The subject did not locate the target in this example. The circles are numbered in the order of attempts made by the subject. The black oval is at the second standard deviation of the uncertainty distribution. (b) An example of Subject A's probes when uncertainty was shown. In this case, the subject followed the uncertainty distribution and successfully localized the target.

Figures 3.11(a) and 3.11(b) show an example of attempts made by a subject (denoted "A") without uncertainty (in blue) and with uncertainty (in red). This subject was able to locate the target using uncertainty but was unable without uncertainty.

Figure 3.12(a) and 3.12(b) show an example in which another subject (denoted "B") was unable to locate the target using uncertainty.

\subsubsection{Hypothesis 2: Fewer incomplete tasks with uncertainty}

A Mann-Whitney test showed that the number of failures was reduced significantly when uncertainty is visualized $p<0.01$. The mean number of incomplete tasks was $3.1 \pm 0.3 \mathrm{SE}$ without uncertainty visualized and $1.9 \pm 0.2 \mathrm{SE}$ with uncertainty 


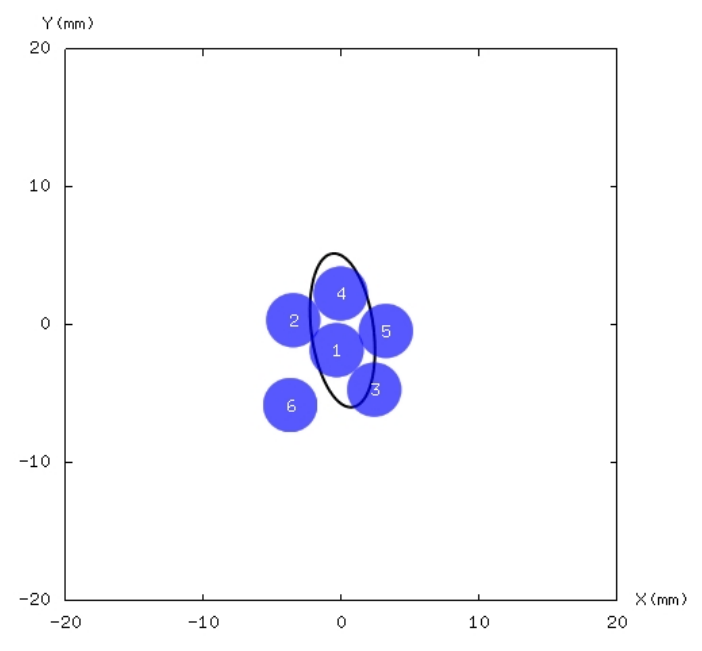

(a)

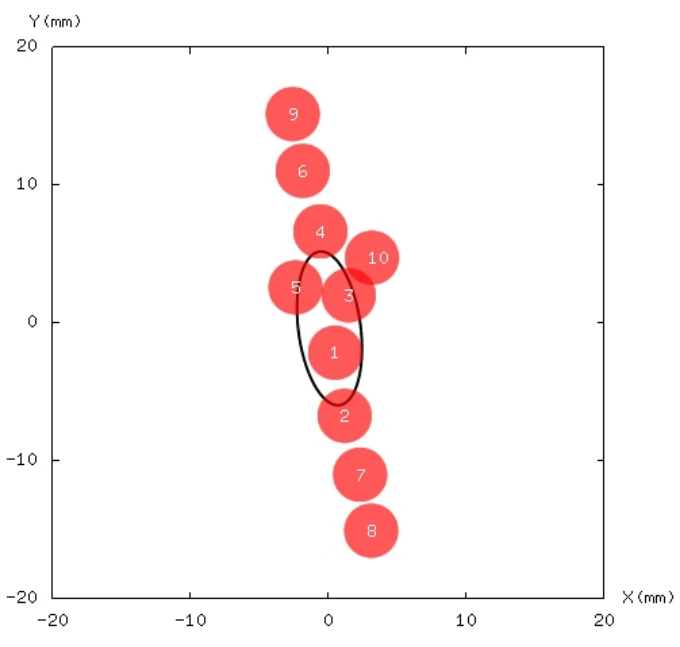

(b)

Figure 3.12: (a) An example of Subject B's probes when uncertainty was not shown. The subject was successful, despite the lack of uncertainty information. (b) An example of Subject B's probes when uncertainty was shown. In this case, the subject followed the uncertainty distribution and did not localize the target.

visualized.

These results are strong evidence that subjects failed less often when uncertainty is visualized.

\subsubsection{Hypothesis 3: Greater fraction of the target probability distribu- tion is covered}

In order to determine whether subjects understood the uncertainty visualization and used the uncertainty distribution to guide their search, we considered the locations of all of the probe attempts that were captured using the tracking system. Note that the same uncertainty distribution was used for each test, but was presented to the subject in a random orientation for each test. To compare the points, we considered them in the coordinate frame of the uncertainty distribution so that, for our analysis, they 
Table 3.1: Cumulative Fraction of Uncertainty Distribution Covered After Each Attempt

\begin{tabular}{|c|ccc|}
\hline Attempt & $\begin{array}{c}\text { No Uncertainty } \\
\text { mean } \pm \mathrm{SE}\end{array}$ & $\begin{array}{c}\text { Uncertainty } \\
\text { mean } \pm \mathrm{SE}\end{array}$ & $\begin{array}{c}\text { Significance } \\
\text { of difference }\end{array}$ \\
\hline 2 & $0.07 \pm 0.02$ & $0.09 \pm 0.02$ & $p=0.10$ \\
3 & $0.12 \pm 0.03$ & $0.22 \pm 0.03$ & $p<0.0001$ \\
4 & $0.17 \pm 0.03$ & $0.25 \pm 0.03$ & $p<0.0001$ \\
5 & $0.21 \pm 0.03$ & $0.31 \pm 0.03$ & $p<0.0001$ \\
\hline
\end{tabular}

were all aligned. Figures 3.11(a), 3.11(b), 3.12(a), and 3.12(b) show examples of the locations of the probe attempts in the coordinate frame of the common uncertainty distribution. (The subjects did not see the distributions aligned like this.) The points are shown as $4 \mathrm{~mm}$ circles because the target was $4 \mathrm{~mm}$ in diameter.

In order to test Hypothesis 3, we excluded the area covered by the first attempt (where the subjects always failed, as discussed previously) and measured the cumulative fraction of the remaining uncertainty distribution that was covered by subsequent attempts, with and without uncertainty visualization. The results are summarized in Table 3.1.

Interestingly, in the second attempt (made immediately after the first, failed attempt), there was no difference in coverage with or without uncertainty visualization. This might be because the task of locating a target using the interface's orthogonal views was difficult and subjects required two attempts to orient themselves spatially in the visualization. But subsequent attempts were significantly better at covering the uncertainty distribution when the distribution was shown, as seen from the $p$ values for attempts 3,4 , and 5 in Table 3.1. This showed that the subjects did, indeed, use the visualization to guide their searches. 


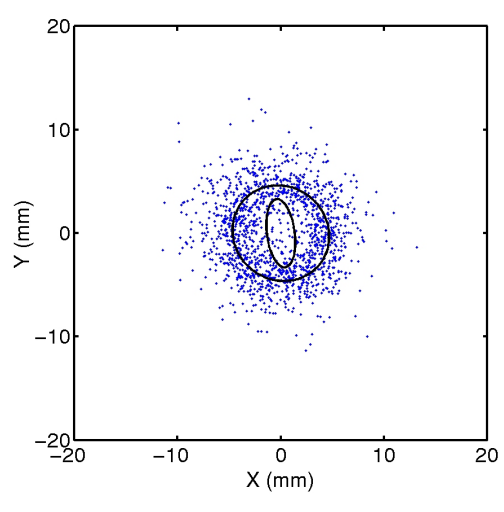

(a)

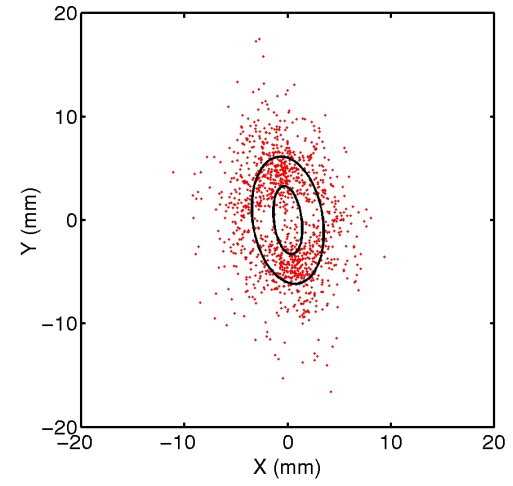

(b)

Figure 3.13: (a) The distribution of displacements of points touched without uncertainty visualized. The smaller ellipse is the covariance of the actual uncertainty distribution. The larger ellipse (almost a circle) is the covariance of the attempts that subjects made. Intuitively, the large ellipse is very circular because subjects did not have an uncertainty visualization to guide their search. (b) The distribution of displacements of points touched with uncertainty visualized. Again the smaller ellipse is the covariance of the actual uncertainty distribution. The larger ellipse is the covariance of subject's attempts.

Figures 3.13(a) and 3.13(b) plot the distribution of displacements of points touched for all subjects. For each attempt, $i$, the points touched were $p_{1}, p_{2}, \ldots, p_{k_{i}}$ for $k$ trials. The displacement of points touched was $\left(p_{2}-p_{1}\right),\left(p_{3}-p_{1}\right), \ldots,\left(p_{k_{i}}-p_{1}\right)$. Qualitatively, the distribution of attempts was circular without uncertainty visualized and more in line with the uncertainty distribution with uncertainty visualized. In each image, the small ellipse showed the covariance of the actual uncertainty distribution. The larger ellipse showed the covariance of subject attempts.

\subsubsection{Hypothesis 4: Attempts match the actual uncertainty distribution}

Hypothesis 4 tested whether subjects followed the uncertainty distribution in their search for the target. We examined the pattern that resulted from subjects attempting 
Table 3.2: Box's M Test Results for Equality of Covariances for Each Subject

\begin{tabular}{|cc|}
\hline Number of subjects & Significance \\
\hline$p<0.10$ & 25 of 30 \\
$p<0.05$ & 18 of 30 \\
$p<0.01$ & 13 of 30 \\
\hline
\end{tabular}

to find the target, to see whether that pattern was consistent with the shape of the uncertainty visualization. To test Hypothesis 4, we used Box's M test which determines the equality of covariance matrices [89]. We compared the covariance of the pattern of the subject's attempts to locate the target and with the covariance of the actual uncertainty distribution (the basis of the visualization). This test simply indicated whether subjects chose a different pattern of attempts when uncertainty was and was not shown.

Table 3.2 summarizes these results. At $10 \%$ significance, 25 of 30 subject's pattern of attempts were different when uncertainty was shown. At $5 \%$ significance, 18 of 30 subject's pattern of attempts were different when uncertainty was shown. At $1 \%$ significance, 13 of 30 subject's pattern of attempts were different when uncertainty was shown. We compared the pooled results of all subjects and tested the equality of the covariances of the pooled results. The covariances were significantly different $(p<0.01)$.

We also compared the square root of the principal components of the covariances of these two ellipses (see Table 3.3). Without uncertainty visualization, the ratio of the major and minor axes of the ellipse was close to 1.0, which means that the subjects were sampling the visualization isotropically (in a circular pattern). With uncertainty visualization, subjects sampled anisotropically (in an elliptical pattern). 
Table 3.3: Comparison of Ratios of Standard Deviations in Principal Directions

\begin{tabular}{|c|c|}
\hline distribution & semimajor/semiminor axis \\
\hline actual uncertainty distribution & 2.5 \\
if uncertainty shown & 1.8 \\
if no uncertainty shown & 1.1 \\
\hline
\end{tabular}

The ratio when uncertainty was shown was much larger than when uncertainty was not shown, which was further evidence that subjects used the visualization to guide their search.

From these figures, it is clear that the subjects understood the visualization. We expected them to probe the "high value" central parts of the distribution before going outside the center, which the analysis suggested they did. However, the subjects might have needed more information for guiding their search in the optimal direction. It seems that some of the subjects did not interpret the visualization as intended, which was likely the result of under-training subjects.

\subsection{Discussion}

We demonstrated a simple method to determine the variation caused by registration uncertainty in a planned linear path. We visualized the uncertainty with a path uncertainty volume. The visualization method resulted in a statistically significant reduction in the number of attempts required to localize a target, and a statistically significant reduction in the number of targets that the pool of subjects failed to localize. The results suggested that users used the visualization to guide their search for the target. However, the magnitude of the uncertainty could have been conveyed to the subject better (see Table 3.3). 
Note that we elected to limit the number of attempts, which may have underestimated the statistical significance of the results because it was likely that subjects would require more attempts to successfully locate the target.

Visualization of registration uncertainty allowed us see unusual and perhaps unexpected features in the distribution of the paths. For example, Figure 3.5 shows that the distribution had an hourglass shape and that the distribution was elongated near the target (see also Figure 3.4). As another example, Figure 3.6 demonstrates a twist in the path distribution.

There are many interacting sources of uncertainty in a computer-assisted surgical system and we have only considered registration uncertainty in this chapter. There are many opportunities for investigating how the various sources of uncertainty affect navigational guidance, which will be discussed in subsequent chapters.

The task defined in the user study may not be representative of how uncertainty would be used in the operating room. Indeed, when we asked a surgical colleague what he would do if he missed an osteoid osteoma in a computer-assisted procedure, his reply was that he would use a bigger drill bit. It may be that surgeons use an uncertainty visualization to decide whether the magnitude of uncertainty is acceptable enough to continue or if, for example, an instrument re-calibration is necessary. Alternatively, the surgeon could use the distribution to avoid critical areas. This study suggested that the confidence interval visualization would be correctly interpreted for that task, too.

Given the promising results of the study, I believe that uncertainty visualization techniques will improve the practice of computer-assisted surgery procedures. 


\section{Chapter 4}

\section{Computing Intraoperative Uncertainty}

This chapter focuses on uncertainty computation techniques that can be used in real time, in the operating room. This work is motivated by several factors. First, it is well known that measurement errors in an optical tracking system are anisotropic, greater in the viewing direction of the camera than in directions perpendicular to the viewing direction. Tracking systems typically use an isotropic least-squares estimate that assumes errors are equal in all directions. Second, jitter ${ }^{1}$ alone in the position of an active target tracked by a Polaris tracking system (Northern Digital Inc., Waterloo, Ontario, Canada) can be as high as $0.35 \mathrm{~mm}$ [43]. Third, these tracking errors will propagate through calibration. The effect on calibration of tracking uncertainty has not been studied. Methods for computing uncertainty in the calibration of tools in computer-aided surgery have not been previously studied. Furthermore, the ramifications of disregarding uncertainty in tracking and calibration have not, to my best knowledge, been studied.

In this chapter, I propose tracking and calibration methods using Kalman filters

\footnotetext{
${ }^{1}$ Tracking jitter is the fluctuation of a fixed target. Jitter is determined by repeatedly sampling a fixed target.
} 
that incorporate uncertainty information. Most notably, I show that these methods achieve reasonable estimates of uncertainty. The main results presented in this chapter are:

- A Kalman filter is proposed in Section 4.2 for tracking a tool in three dimensions. The filter reports a covariance (uncertainty) estimate for each pose computed by the filter.

- I establish through simulated and real data that this method has sufficient tracking accuracy and that the computed covariance estimates are in agreement with the true covariances.

- A Kalman filter is proposed for tool calibration in Section 4.3. The filter reports a covariance estimate for the calibration parameters.

- I validate the computed calibration covariance by comparison to a bootstrapped covariance of the least-squares solution of calibration.

- In Section 4.4, I describe a dual Kalman filter that propagates the uncertainty from tracking to calibration.

Estimating pose and calibration parameters using a Kalman filter is not a new idea to computer vision and robotics research (see, for example Ayache and Faugeras [5], Gennery [30], Kraft [47], and LaViola [53]). However, using the covariance estimate produced by the filter as a measurement of uncertainty in the camera system is, to my best knowledge, an unexplored area. The main contribution is to demonstrate that a reliable estimate of the covariance of target pose can be computed and can be applied, for example, to show uncertainty in the path of a surgical tool or to propagate through calibration. 


\subsection{UKF Target Tracking Algorithm I}

In this section, I describe an unscented Kalman filter (UKF) for estimating the pose (position and orientation) of a target with known IRED locations based on the tracking filter developed by Welch and Bishop [116]. Welch and Bishop developed a UKF for tracking in which the rotational elements of pose were stored as Euler angles and computed using incremental estimates of orientation (the orientation difference between time steps) rather than the absolute orientation. Absolute orientation was determined and stored as a quaternion outside of the filter. Tracking the incremental difference in Euler angles avoided inconsistencies at $\pm \pi$ and permitted linear approximations.

The filter algorithm used the predicted position of IRED locations to find the actual IRED locations in space; these measurements updated the estimates of position, incremental orientation, linear velocity, and incremental angular velocity. Inputs to the filter were the individual IRED locations and outputs were the pose (position and orientation) of the target and a covariance estimate of the pose. The method assumed that the uncertainty over the measurement volume was constant.

The state vector described the target position and orientation at any time $t$. The state vector was

$$
\mathbf{x}=[x, y, z, \dot{x}, \dot{y}, \dot{z}, \phi, \theta, \psi, \dot{\phi}, \dot{\theta}, \dot{\psi}]^{T}
$$

where $(x, y, z)$ represented target position in Cartesian coordinates in $\mathrm{mm},(\phi, \theta, \psi)$ incremental orientation in radians, $(\dot{x}, \dot{y}, \dot{z})$ linear velocity in $\mathrm{mm} / \mathrm{s}$, and $(\dot{\phi}, \dot{\theta}, \dot{\psi})$ incremental angular velocity in radians/s. The linear and angular velocities were the first derivatives (with respect to time) of the target position and orientation, 
respectively.

The current estimate of target orientation was maintained outside of the filter as a unit quaternion in the form

$$
\alpha=\left(\alpha_{w}, \alpha_{x}, \alpha_{y}, \alpha_{z}\right)
$$

The three state vector components $\phi, \theta, \psi$ of rotation represented the correction needed to the current estimate of absolute orientation (i.e. the quaternion above). The orientations were represented in this manner so that incremental changes in orientation (which are small) could be linearly approximated.

The target motion over time $\Delta t$ was modeled as:

$$
\mathbf{x}_{t+\Delta t}=F\left(\mathbf{x}_{\Delta t}, \mathbf{v}_{\Delta t}\right)
$$

where $\mathbf{v}_{\Delta t}$ was the process noise and $F$ was the process model which projects the state $\mathbf{x}$ forward in time. The process model represented

$$
\begin{aligned}
& \mathbf{x}_{t+\Delta t}=\mathbf{x}_{t}+\dot{\mathbf{x}}_{t} \Delta t \quad \text { (position/orientation components) } \\
& \dot{\mathbf{x}}_{t+\Delta t}=\dot{\mathbf{x}}_{t} \quad \text { (velocity/rotational velocity components) }
\end{aligned}
$$

for each of the elements in Equation 4.1 (note that acceleration was assumed to be zero). The process noise vector $\mathbf{v}_{\Delta t}$ in Equation 4.2 represented uncertainty in the target position and orientation during the time interval $\Delta t$, and was modeled by white Gaussian noise. The $n \times n$ process noise covariance matrix was manually tuned to reasonable values that described the particular tracking configuration. In this case, 
process noise matrix had the form

$\left[\begin{array}{cccccccccccc}2.0^{2} & 0 & 0 & 0 & 0 & 0 & 0 & 0 & 0 & 0 & 0 & 0 \\ 0 & 2.0^{2} & 0 & 0 & 0 & 0 & 0 & 0 & 0 & 0 & 0 & 0 \\ 0 & 0 & 0.3^{2} & 0 & 0 & 0 & 0 & 0 & 0 & 0 & 0 & 0 \\ 0 & 0 & 0 & \left(\frac{3 \pi}{180}\right)^{2} & 0 & 0 & 0 & 0 & 0 & 0 & 0 & 0 \\ 0 & 0 & 0 & 0 & \left(\frac{3 \pi}{180}\right)^{2} & 0 & 0 & 0 & 0 & 0 & 0 & 0 \\ 0 & 0 & 0 & 0 & 0 & \left(\frac{3 \pi}{180}\right)^{2} & 0 & 0 & 0 & 0 & 0 & 0 \\ 0 & 0 & 0 & 0 & 0 & 0 & \left(\frac{5 \pi}{180}\right)^{2} & 0 & 0 & 0 & 0 & 0 \\ 0 & 0 & 0 & 0 & 0 & 0 & 0 & \left(\frac{5 \pi}{180}\right)^{2} & 0 & 0 & 0 & 0 \\ 0 & 0 & 0 & 0 & 0 & 0 & 0 & 0 & \left(\frac{5 \pi}{180}\right)^{2} & 0 & 0 & 0 \\ 0 & 0 & 0 & 0 & 0 & 0 & 0 & 0 & 0 & \left(\frac{5 \pi}{180}\right)^{2} & 0 & 0 \\ 0 & 0 & 0 & 0 & 0 & 0 & 0 & 0 & 0 & 0 & \left(\frac{5 \pi}{180}\right)^{2} & 0 \\ 0 & 0 & 0 & 0 & 0 & 0 & 0 & 0 & 0 & 0 & 0 & \left(\frac{5 \pi}{180}\right)^{2}\end{array}\right]$

where the diagonals corresponded to the noise in elements $x, y, z, \dot{x}, \dot{y}, \dot{z}, \phi, \theta, \psi, \dot{\phi}, \dot{\theta}, \dot{\psi}$ of the state vector.

The measurement model described the ideal response of the tracking system in the absence of noise for all of the IREDs available from the camera at time $t$. In this case, the measurement model was simply the centroid of a four-marker target. I tuned the measurement noise covariance based on a reasonable guess of the magnitude of the noise supplied by the specifications for the simulated camera model. The noise 
covariance matrix (with units in millimeters) was

$$
\left[\begin{array}{ccc}
0.15^{2} & 0 & 0 \\
0 & 0.5^{2} & 0 \\
0 & 0 & 0.15^{2}
\end{array}\right]
$$

\subsubsection{Experiments}

The purpose of my experiments was to show that I could compute a reliable estimate of uncertainty in target pose. I ran our filter on simulated tracking data and compared this to a set of simulated marker locations that were registered to noisy measurements using a registration technique developed by Ohta and Kanatani [82].

Ohta and Kanatani described an algorithm for estimating the rotation that best aligned two point sets in the presence of anisotropic and inhomogeneous noise. They also defined a covariance matrix suitable for rotations, and showed that their algorithm could estimate their covariance matrix. Their covariance matrix was defined as the expected value $E\left[\Delta \Omega(\Delta \Omega)^{T}\right]$ where $\Delta \Omega$ was the normalized axis of rotation multiplied by the angle of rotation. I compared Ohta and Kanatani's covariance estimate with the state covariance estimate obtained by the UKF to verify the results. I used Ohta and Kanatani's estimate because it assumed an anisotropic noise model.

The simulations oriented the target so that it faced the camera in the $z=$ -2000 mm plane (see Figure 4.1). I simulated moving the target on an ellipse in this plane. The angular velocity about $z$ was constant and equal to $2 \pi / 5 \mathrm{rad} / \mathrm{s}$ (one revolution around the ellipse every five seconds). The ellipse had axes of length $500 \mathrm{~mm}$ in the $x$ direction and $250 \mathrm{~mm}$ in the $y$ direction. To each position measurement, I added noise drawn from a normal distribution with mean zero and covariance 

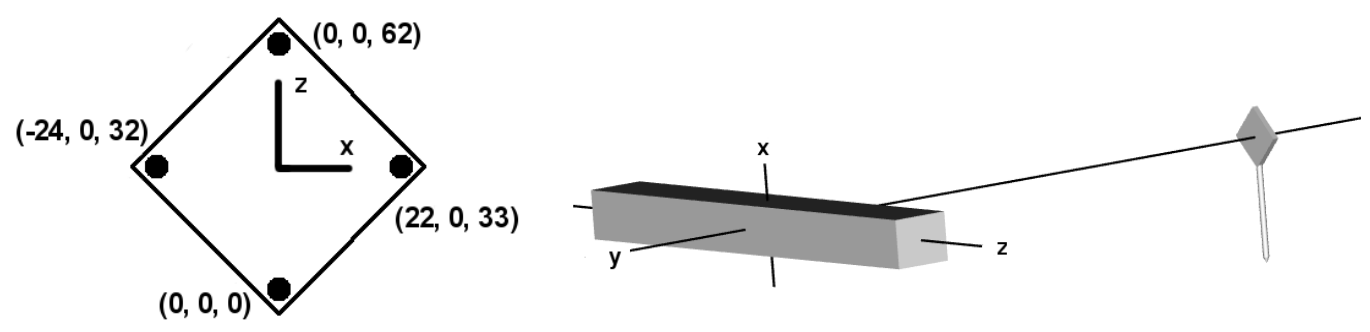

Figure 4.1: Target model (left) and optical tracking system (right) configuration used in the simulations; all units are in millimeters. The target consists of four markers arranged in a quadrilateral. Measurement noise in the viewing direction $-z$ is larger than in the viewing plane. The target is placed in the viewing direction of the camera.

with diagonal entries $(0.15 \mathrm{~mm})^{2},(0.5 \mathrm{~mm})^{2}$, and $(0.15 \mathrm{~mm})^{2}$ representing position which corresponded to the noise covariance in the pose estimation.

For each angle of rotation, I generated 10000 sets of measured marker locations where each location was the model marker location rotated by the angle of rotation with zero-mean, additive Gaussian noise of covariance

$$
\mathbf{N}_{t}=\mathbf{N}=\left[\begin{array}{ccc}
n_{x_{c}}^{2} & 0 & 0 \\
0 & n_{y_{c}}^{2} & 0 \\
0 & 0 & n_{z_{c}}^{2}
\end{array}\right]
$$

where $n_{x_{c}}=n_{y_{c}}$ (isotropic noise in the camera viewing plane), $n_{z_{c}}=s n_{x_{c}}$ for some scalar $s \geq 1$ (anisotropic noise in the viewing direction), and $n_{x_{c}}^{2}+n_{y_{c}}^{2}+n_{z_{c}}^{2}=c$ for a constant value $c=0.1^{2}+0.1^{2}+0.3^{2}$ (constant noise magnitude). All noise variances were in the tracking camera coordinate frame. The simulated marker locations were registered to the noisy measured marker locations using Ohta and Kanatani's method [82] for comparison.

The simulated target consisted of four markers in a quadrilateral configuration. 
The marker coordinates were $\left[\begin{array}{lll}0 & 0 & 0\end{array}\right]^{T},\left[\begin{array}{lll}-24 & 0 & 32\end{array}\right]^{T},\left[\begin{array}{lll}0 & 0 & 62\end{array}\right]^{T}$, and $\left[\begin{array}{lll}22 & 0 & 33\end{array}\right]^{T}$ in millimeters (see Figure 4.1).

I performed these experiments using synthesized Optotrak Certus (Northern Digital Inc., Waterloo, Canada) data. I chose the Optotrak because it reported the raw position of individual IRED locations. I computed the pose of the target directly from IRED locations to capture as much of the uncertainty as possible.

\subsubsection{Validation}

The purpose of the experiments was to show that I could accurately use this method for tracking and compute a reliable estimate of uncertainty in target pose. The filter was run on simulated tracking data and compared to a set of simulated marker locations that were registered to noisy measurements using a registration technique by Ohta and Kanatani [82].

\subsubsection{Results}

In order to establish that the tracking method achieved favorable tracking results, I computed the root-mean-squared (RMS) error of the pose computed by the UKF with respect to the simulated ground truth. Figure 4.2 shows the RMS error of the tracking results for the simulated data. The results indicated that the tracking accuracy was good for my method.

I computed the eigenvalues of the state covariance matrix generated by the UKF and compared them to the Ohta and Kanatani method. The square root of the principal components as a function of rotation angle of both methods are shown in Figure 4.3. The covariance estimate computed by the tracking algorithm was in 

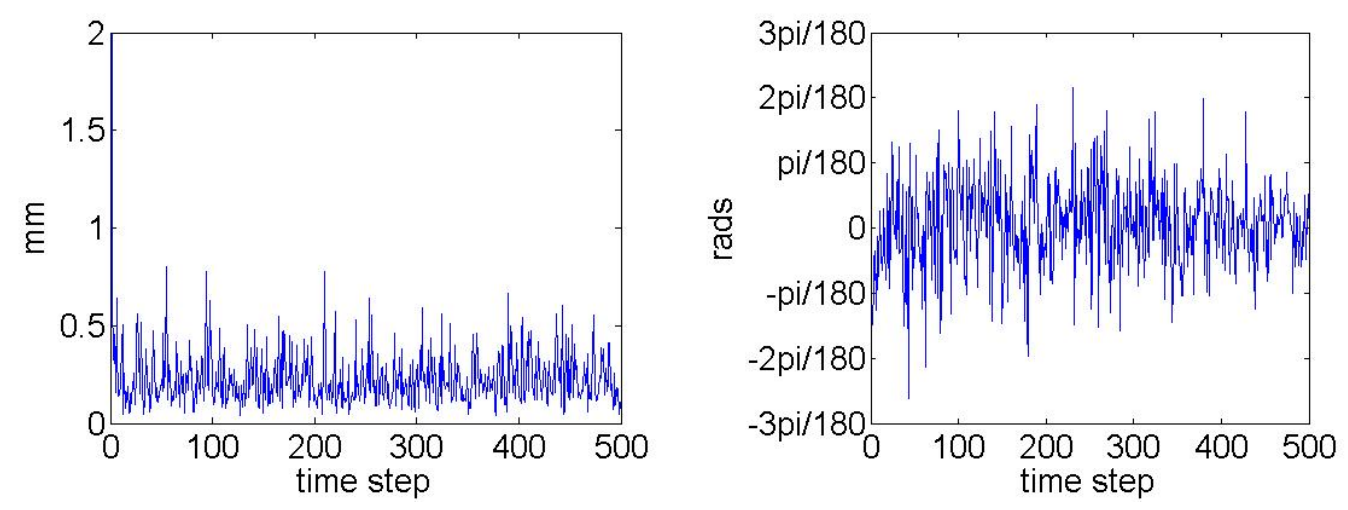

Figure 4.2: Difference in position and orientation computed by UKF tracking method and the simulated correct result. This result indicates that the tracking accuracy was good for my method.

good agreement with the simulation results for rotation about $\mathrm{x}\left(\mu_{x}=0.0 \pm 0.0\right), \mathrm{y}$ $\left(\mu_{y}=0.0 \pm 0.0\right)$, and $\mathrm{z}\left(\mu_{z}=0.0 \pm 0.1\right)$.

I ran a second simulation with 50 data points removed to see if the filter could recover the covariance estimate quickly once the data stream resumed from the camera system. Figure 4.4 demonstrates that the covariance estimate recovered within approximately five filter steps even if the previous estimate of the filter was poor.

\subsubsection{Discussion}

The proposed method converged quickly, making real-time estimates and visualizations possible. Moreover, the tracking filter computed the pose based on measurements as they became available from the tracking system, thus compensating for obscured IREDs and latency in data acquisition. Most interesting was that the simulations demonstrated that the covariance estimate produced by the algorithm agreed with lower bounds for the covariance established by Ohta and Kanatani [82]. 

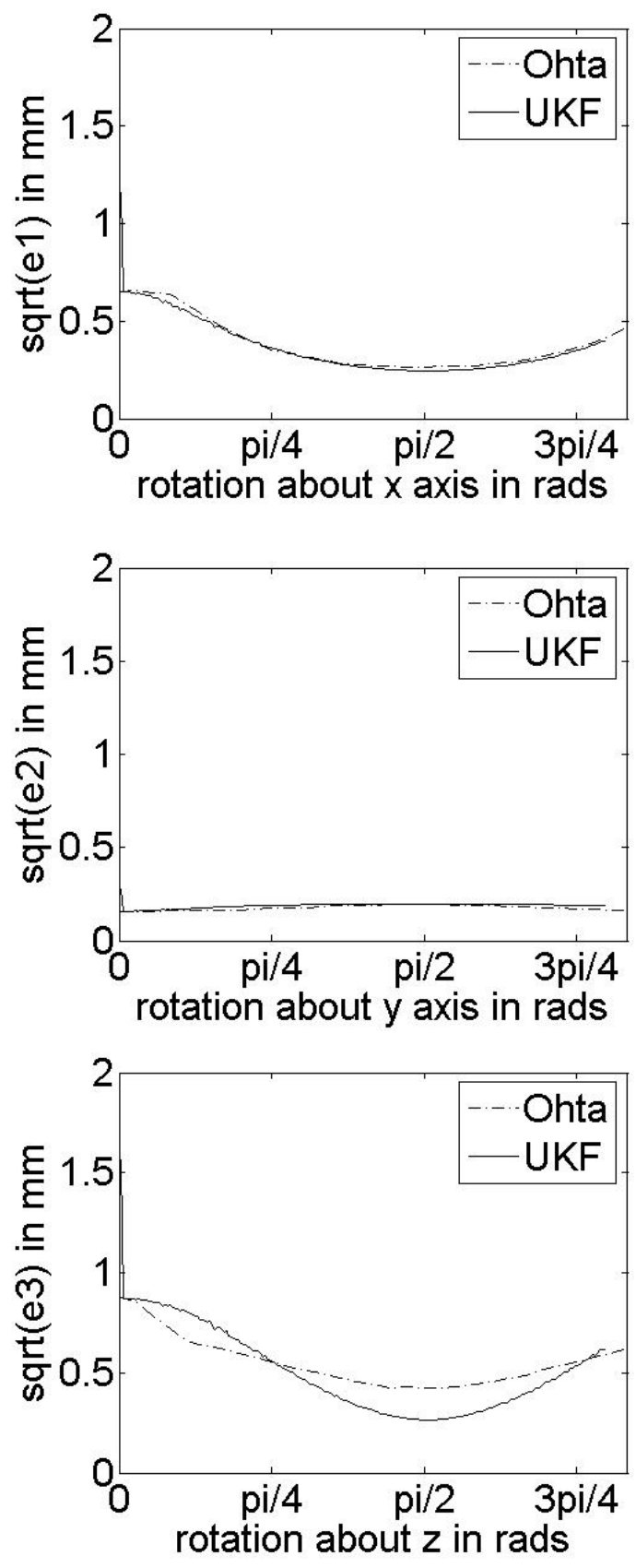

Figure 4.3: Simulation results for the target using the incremental orientation approach of Welch and Bishop [116] that showed the rotational variance about the $x$ (top), $y$ (middle), and $z$ (bottom) axes, obtained from the square root of the first principal component of the rotational error covariance matrix. The result (solid line) compared favorably with the lower bounds (dashed line) of Ohta and Kanatani. 

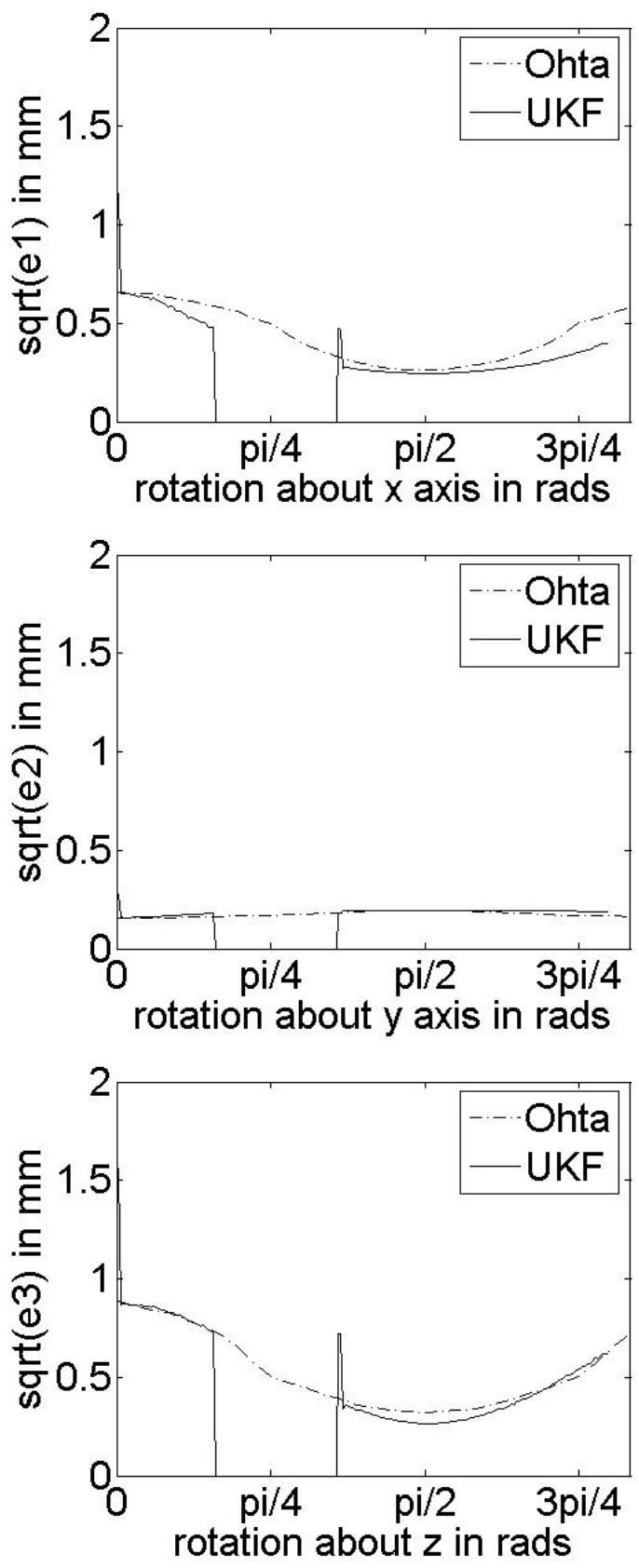

Figure 4.4: Simulation results for the target after removing data from the simulation. This demonstrated that the covariance estimate recovers well within a few filter steps, even in the absence of a good previous estimate. 
However, even though my method had very good tracking accuracy and uncertainty estimation, it was fundamentally flawed with respect to computing intraoperative uncertainty. I chose to represent orientation using Euler angles. In order to avoid discontinuities at $\pm \pi$, I tracked the incremental orientation (the orientation difference between time steps), a technique inspired by Welch and Bishop [116], rather than the absolute orientation. Therefore, the covariance estimate was based on incremental orientation rather than absolute orientation. Unfortunately, I was unaware of a method that would convert the many incremental orientation covariance estimates to a single absolute orientation covariance estimate.

An estimate of absolute orientation uncertainty was needed in order to have a meaningful estimate of intraoperative uncertainty. Toward that end, the second approach was to track the quaternion directly based on work by Kraft [47]. In Kraft's method, the state vector stored the orientation (as a quaternion) and angular velocity (in rotation-vector representation). Kraft converted the quaternion to rotation-vector representation within the process and measurement models. The UKF equations (the originals are shown in Appendix A) were modified for quaternion space: scalar multiplication became quaternion multiplication, mean became quaternion mean, and so on. Using simulated and real data, my implementation of the Kraft filter was not able to track with sufficient accuracy (tracking error was on the order of $3 \mathrm{~mm}$, significantly worse than simply tracking with the isotropic method supplied by the manufacturer of the tracking system). The covariance estimates were not valid. This may not be such a surprise given that the original work never validated the covariance estimate or showed tracking with real data. 


\subsection{UKF Tracking Algorithm II}

In this section, I propose a target tracking algorithm that represents orientation using rotation vectors. This algorithm takes as input four marker (individual IRED) locations and outputs the pose of the target and a covariance estimate of the pose.

For my method, the state model was

$$
\mathbf{x}_{t+1}=F\left(\mathbf{x}_{t}, \mathbf{u}_{t}\right)+\mathbf{v}_{t}
$$

where

$$
\mathbf{x}_{t}=[x, y, z, \dot{x}, \dot{y}, \dot{z}, \phi, \theta, \psi, \dot{\phi}, \dot{\theta}, \dot{\psi}]^{T}
$$

was the state vector, $(x, y, z)$ was target position $(\mathrm{mm}),(\phi, \theta, \psi)$ orientation (rads), $(\dot{x}, \dot{y}, \dot{z})$ linear velocity $(\mathrm{mm} / \mathrm{s})$, and $(\dot{\phi}, \dot{\theta}, \dot{\psi})$ angular velocity (rads/s) at time $t$.

The orientation was represented using rotation vectors. Given a unit quaternion $q=q_{w}, q_{x}, q_{y}, q_{z}$, the rotation vector had the form

$$
q=\cos (\theta / 2)+\mathbf{e} \sin (\theta / 2)
$$

where $\theta$ was the rotation angle and $\mathbf{e}=x, y, z$ was the rotation axis as a unit vector.

The process model updated the state vector according to:

$$
\begin{aligned}
& \mathbf{x}_{t+1}=\mathbf{x}_{t}+\dot{\mathbf{x}}_{t} \Delta t+\mathbf{v}_{t} \quad \text { (position/orientation components) } \\
& \dot{\mathbf{x}}_{t+1}=\dot{\mathbf{x}}_{t}+\mathbf{v}_{t} \quad(\text { velocity/rotational velocity components) }
\end{aligned}
$$

The linear and angular velocities were the first derivatives of the target position and 
orientation, respectively. The process noise was drawn from zero-mean Gaussian noise with covariance $\mathbf{v}_{t}$. Determining the process noise covariance was difficult because I cannot observe the process directly. These parameters can be estimated by running the filter offline, on simulated data [114]. For the filter, the process noise matrix had the form

\begin{tabular}{|c|c|c|c|c|c|c|c|c|c|c|c|}
\hline $2.0^{2}$ & 0 & 0 & 0 & 0 & 0 & 0 & 0 & 0 & 0 & 0 & 0 \\
\hline 0 & $2.0^{2}$ & 0 & 0 & 0 & 0 & 0 & 0 & 0 & 0 & 0 & 0 \\
\hline 0 & 0 & $3.0^{2}$ & 0 & 0 & 0 & 0 & 0 & 0 & 0 & 0 & 0 \\
\hline 0 & 0 & 0 & $\left(\frac{3 \pi}{180}\right)^{2}$ & 0 & 0 & 0 & 0 & 0 & 0 & 0 & 0 \\
\hline 0 & 0 & 0 & 0 & $\left(\frac{3 \pi}{180}\right)^{2}$ & 0 & 0 & 0 & 0 & 0 & 0 & 0 \\
\hline 0 & 0 & 0 & 0 & 0 & $\left(\frac{3 \pi}{180}\right)^{2}$ & 0 & 0 & 0 & 0 & 0 & 0 \\
\hline 0 & 0 & 0 & 0 & 0 & 0 & $\left(\frac{5 \pi}{180}\right)^{2}$ & 0 & 0 & 0 & 0 & 0 \\
\hline 0 & 0 & 0 & 0 & 0 & 0 & 0 & $\left(\frac{5 \pi}{180}\right)^{2}$ & 0 & 0 & 0 & 0 \\
\hline 0 & 0 & 0 & 0 & 0 & 0 & 0 & 0 & $\left(\frac{5 \pi}{180}\right)^{2}$ & 0 & 0 & 0 \\
\hline 0 & 0 & 0 & 0 & 0 & 0 & 0 & 0 & 0 & $\left(\frac{5 \pi}{180}\right)^{2}$ & 0 & 0 \\
\hline 0 & 0 & 0 & 0 & 0 & 0 & 0 & 0 & 0 & 0 & $\left(\frac{5 \pi}{180}\right)^{2}$ & 0 \\
\hline 0 & 0 & 0 & 0 & 0 & 0 & 0 & 0 & 0 & 0 & 0 & $\left(\frac{5 \pi}{180}\right)^{2}$ \\
\hline
\end{tabular}

where the diagonals corresponded to the noise in elements $x, y, z, \dot{x}, \dot{y}, \dot{z}, \phi, \theta, \psi, \dot{\phi}, \dot{\theta}, \dot{\psi}$ of the state vector.

The observation model simply applied the transformation matrix described by the position $(x, y, z)$ and orientation $(\phi, \theta, \psi)$ components of the state $\mathbf{x}_{k}$ to the target model location $\mathbf{p}_{i}$. The observation model for $i$ measurements for each point $\mathbf{m}_{i}$ in 
camera coordinates was:

$$
\mathbf{m}_{i}=R(\phi, \theta, \psi) \mathbf{p}_{i}+\mathbf{t}(x, y, z)+\mathbf{n}_{i}
$$

where $R$ was the rotation matrix, $\mathbf{t}$ was the translation, and $\mathbf{n}_{i}$ was the measurement noise. The measurement noise covariance represented any random error, such as electrical noise, in the measurement. Measurement noise can be determined from manufacturer design specifications or confirmed empirically by sampling off-line measurements to determine the variance in the measurement noise [116]. The measurement noise covariance was tuned based on a reasonable guess of the magnitude of the noise supplied by the manufacturer's specifications for the camera system (see [81]). The measurement covariance matrix was

$$
\left[\begin{array}{ccc}
0.15^{2} & 0 & 0 \\
0 & 0.15^{2} & 0 \\
0 & 0 & 0.5^{2}
\end{array}\right]
$$

\subsubsection{Experiments}

Again I wanted to show that the filter produced reliable estimates of pose, and more interestingly, reliable estimates of uncertainty. Ideally, I would have tested the algorithm on real data where the target's true position was determined by a coordinate measurement machine. Unfortunately, I did not have access to this type of machine. Instead, I chose to 'cleanse' real data and contaminate that data with noise. 
I computed noise-free IRED locations from tracking data from an Optotrak Certus (Northern Digital Inc, Waterloo, Canada). To do this, I used the pose computed by the software provided by the manufacturer and applied this to the target model to get the ground-truth noise-free IRED locations. I added noise drawn from a normal distribution with mean zero and covariance with diagonal entries $(0.15 \mathrm{~mm})^{2}$, $(0.15 \mathrm{~mm})^{2}$, and $(0.5 \mathrm{~mm})^{2}$ back to the noise-free IREDs . I ran the simulated tracking data on 10000 sets of the simulated noisy IRED data where each set contained 250 tracked poses.

\subsubsection{Validation}

For comparison, I used an algorithm by Matei and Meer [65] for optimal rigid motion estimation under heteroscedastic noise. An optimal pose solution could be computed through a linearizing algorithm in quaternion space if covariances matrices were known for two sets of matched 3D measurements. Rotation and translation parameters were estimated by solving a "heteroscedastic errors in variables" (HEIV) regression [65]. HEIV regression was advantageous because it converged to an optimal estimate within two or three iterations. I ran HEIV regression on 10000 sets of simulated noisy IRED data.

\subsubsection{Results}

I compared the UKF tracking estimates with the simulated ground truth by computing the absolute difference in the position and orientation. Figure 4.5 plots the absolute difference. The maximum difference was $0.7 \mathrm{~mm}$ for position and $0.3^{\circ}$ for 

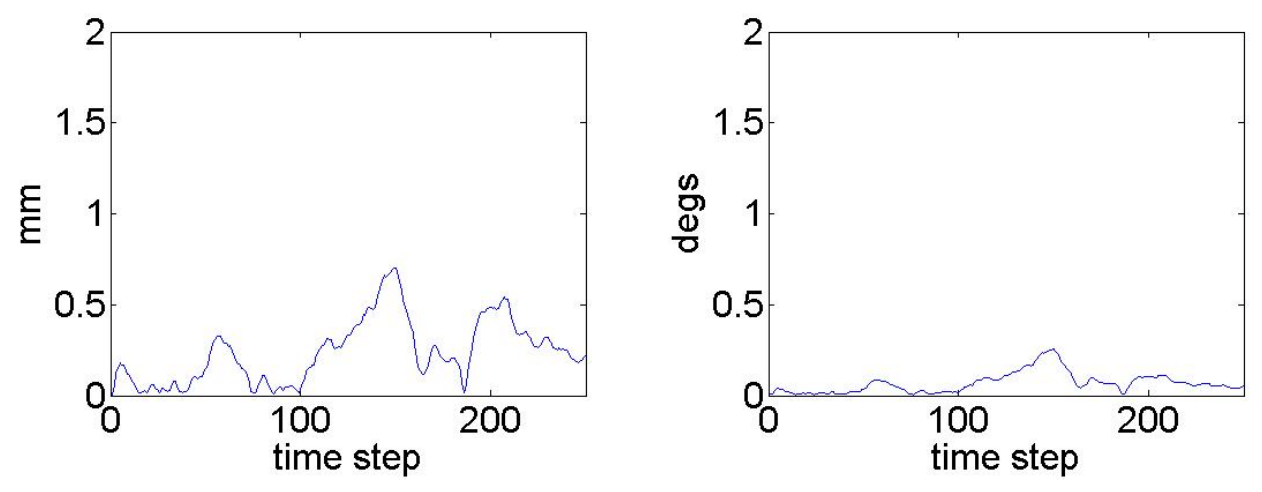

Figure 4.5: Difference in position and orientation computed by UKF tracking method and the simulated correct result. This result indicated that the tracking accuracy is quite acceptable for the method.

orientation, well within the range of accuracy of current tracking technologies. I computed the covariance of the 10000 HEIV trials and compared this to the covariance computed by the UKF method. Figure 4.6 shows the square root of the eigenvalues $e 1, e 2, e 3, e 4, e 5$, and $e 6$ for the UKF plotted against the covariance of the HEIV trials. The mean and standard deviation of the difference of the two curves for each eigenvalue was

$$
\left[\begin{array}{l}
e 1 \\
e 2 \\
e 3 \\
e 4 \\
e 5 \\
e 6
\end{array}\right]=\left[\begin{array}{l}
0.02 \\
0.01 \\
0.01 \\
0.0 \\
0.0 \\
0.0
\end{array}\right] \pm\left[\begin{array}{l}
0.04 \\
0.06 \\
0.06 \\
0.06 \\
0.06 \\
0.06
\end{array}\right] .
$$



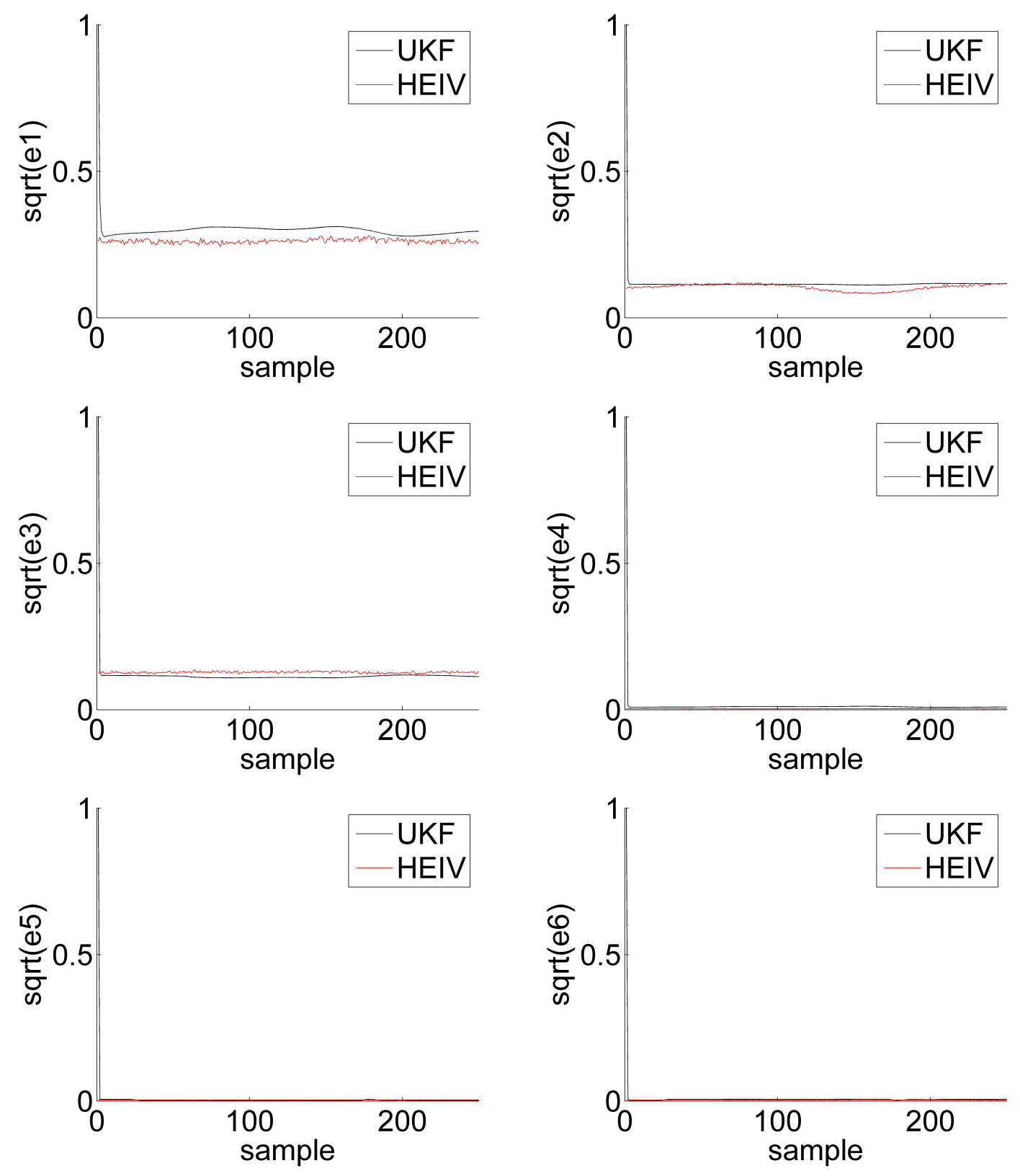

Figure 4.6: The graphs show the square root of the diagonal elements of the eigenvalues of the covariance matrix. Individual graphs show the HEIV and UKF results for the square root of the eigenvalues $e 1, e 2, e 3, e 4, e 5$, and $e 6$. The results indicated that the covariance estimates from the tracking filter were in agreement with the covariances computed from the HEIV estimates. 


\subsubsection{Discussion}

The tracking UKF took as input raw IRED locations from the tracking system and computed reasonable tracking and covariance estimates for each measurement, as they became available from the tracking system. I used the raw IRED locations because they enabled me to capture uncertainty directly from the measurement source rather than introducing further uncertainty from the pose calculation used by the manufacturer.

I chose to represent orientation using rotation-vector notation which enabled me to compute an absolute orientation estimate of pose. It is important to note that for rotation vectors, singularities exist at 0 radians and $\pi$ radians where the axis jumps for small changes in input (for example, at 0 radians the axis is arbitrary, any axis will produce the same result). It is necessary to compensate for this in the filter implementation.

The results showed that the covariance computed by the method was close to the true covariance. Therefore, it was possible to compute a reliable estimate of tracking uncertainty in real time as measurements became available from the tracking system.

\subsection{UKF Calibration Algorithm}

I computed the location of the tip of the probe in camera coordinates using a UKF for calibration. The calibration was achieved by rotating the tool about a fixed pivot point. The calibration algorithm took as input $n$ points on a sphere, fit these data to a sphere, and output the radius and center of the sphere as well as a covariance estimate of these. The center of the sphere was the location of the tip and the radius is the length of the tool from the target to the tip; both were measured in camera 
coordinates. Appendix A describes the UKF parameter estimation. The notation in this section follows the convention in the appendix.

The parameter model was $\mathbf{w}_{t+1}=\mathbf{w}_{t}+\mathbf{r}_{t}$ where $\mathbf{w}_{t}=\left[x_{c}, y_{c}, z_{c}, r\right]_{t}^{T}$ was the vector of calibration parameters of a sphere with center $x_{c}, y_{c}, z_{c}$ and radius $r$ at time $t$. The uncertainty of the parameter estimation was drawn from zero-mean Gaussian noise with covariance $\mathbf{r}_{t}$. The process model was simply the translational elements of pose from the tracking system. The process noise covariance had diagonal entries equal to $(0.0005 \mathrm{~mm})^{2}$.

The observations for $n$ measurements was

$$
\mathbf{d}_{t}=\left[\begin{array}{c}
G_{1} \\
\vdots \\
G_{n}
\end{array}\right]=\left[\begin{array}{ccc}
x_{c_{1}}+r \frac{\Delta x}{\Delta r} & y_{c_{1}}+r \frac{\Delta y}{\Delta r} & z_{c_{1}}+r \frac{\Delta z}{\Delta r} \\
\vdots & \vdots & \vdots \\
x_{c_{n}}+r_{n} \frac{\Delta x}{\Delta r} & y_{c_{n}}+r_{n} \frac{\Delta y}{\Delta r} & z_{c_{n}}+r_{n} \frac{\Delta z}{\Delta r}
\end{array}\right]+\mathbf{e}_{t}
$$

where

$$
\begin{array}{cc}
\Delta x & =x-x_{c_{j}} \\
\Delta y & =y-y_{c_{j}} \\
\Delta z & =z-z_{c_{j}} \\
\Delta r & =\sqrt{(\Delta x)^{2}+(\Delta y)^{2}+(\Delta z)^{2}}
\end{array}
$$

and $G_{t}$ was the $j^{\text {th }}$ measured point on the sphere and $\mathbf{e}_{t}$ was the measurement noise. The observation covariance $\mathbf{r}_{t}$ was chosen based on a reasonable guess of the measurement noise magnitude. In this case, all of the diagonals were equal to $(\sqrt{3} / 4 \mathrm{~mm})^{2}$. An initial estimate $\mathbf{w}_{0}$ was obtained using a least-squares sphere fitting algorithm [18].

\subsubsection{Experiments}

I performed three types of calibration:

Type 1 The probe was moved to maximize the amount of coverage of the sphere. 
Type 2 The probe was restricted to sampling points on the sphere primarily in the $y$ and $z$ axes.

Type 3 The probe was restricted to movement in $x$ and $z$.

I computed 100 sets of each calibration type using the UKF calibration method and a least-squares sphere fitting algorithm [18]. After every $25^{\text {th }}$ calibration sample, the probe was moved to another place inside the measurement volume. During each individual calibration, 500 data points were collected. I collected all of the data with an Optotrak Certus. In the Optotrak system, the positive $x$ axis faced up, the positive $y$ axis faced to the right, and the $z$ axis was in line with the viewing direction. The probe was facing the camera in the $-z$ direction. The probe was manually aligned with $y=0$ and $x=0$.

\subsubsection{Results}

In order to determine whether the calibration achieved reliable parameter estimates, I compared the results of the UKF with a least-squares sphere fit. Figure 4.7 compares the RMS of the tip location estimated by the UKF method to a least-squares method for the three calibration data sets. The mean difference in the tip location was $0.1 \pm 0.1 \mathrm{~mm}$ for all three calibration types. Figure 4.8 plots the square root of the eigenvalues e1, e2, e3, and e4 computed by the calibration method as a function of the sampled points over all trials for the three calibration data sets. These results demonstrated a general decrease in the uncertainty as more points were collected by the calibration method.

I estimated the covariance of each of the least-squares calibration estimates using the bias-corrected and accelerated bootstrap method [19] (the bootstrap statistic is 

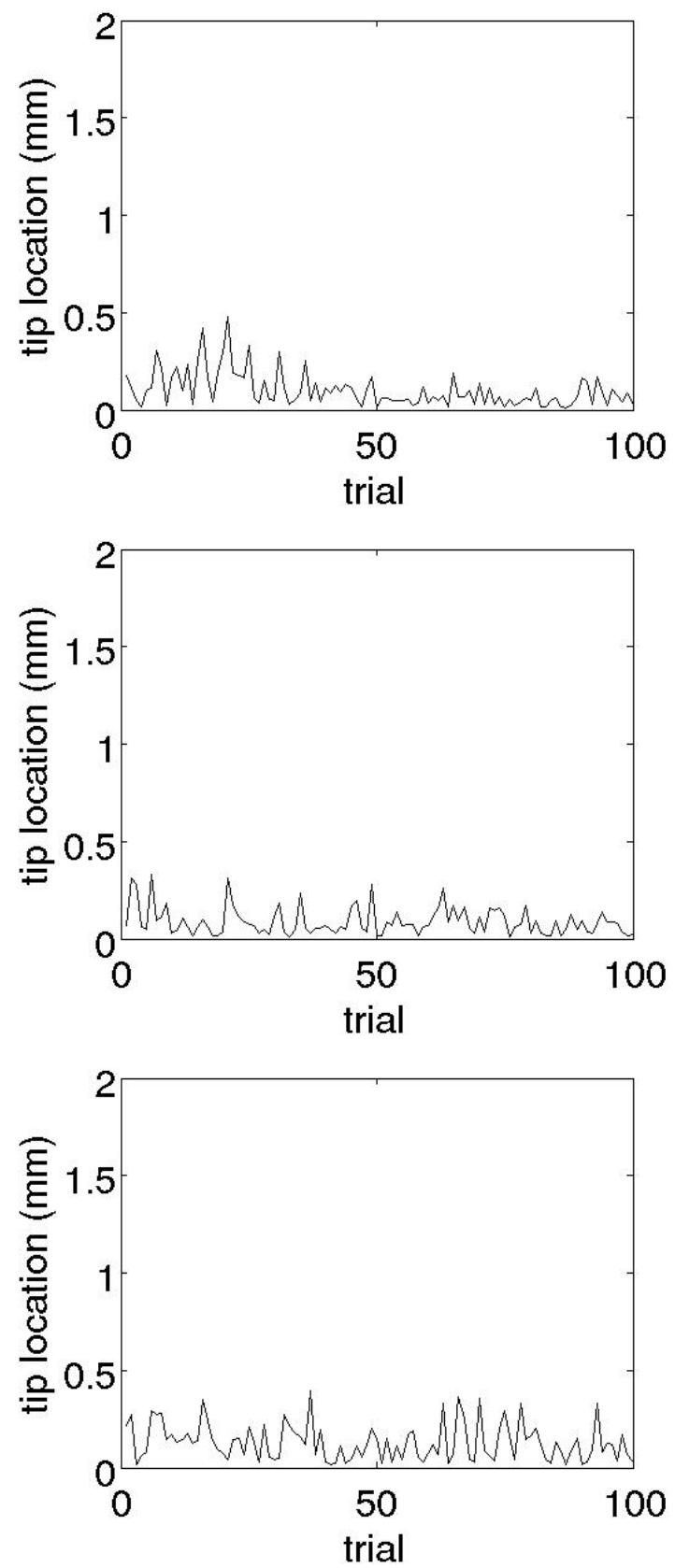

Figure 4.7: Difference in tip location (x,y,z) computed by UKF calibration method and least-squares solution for all three calibration data sets. Results were in good agreement. 

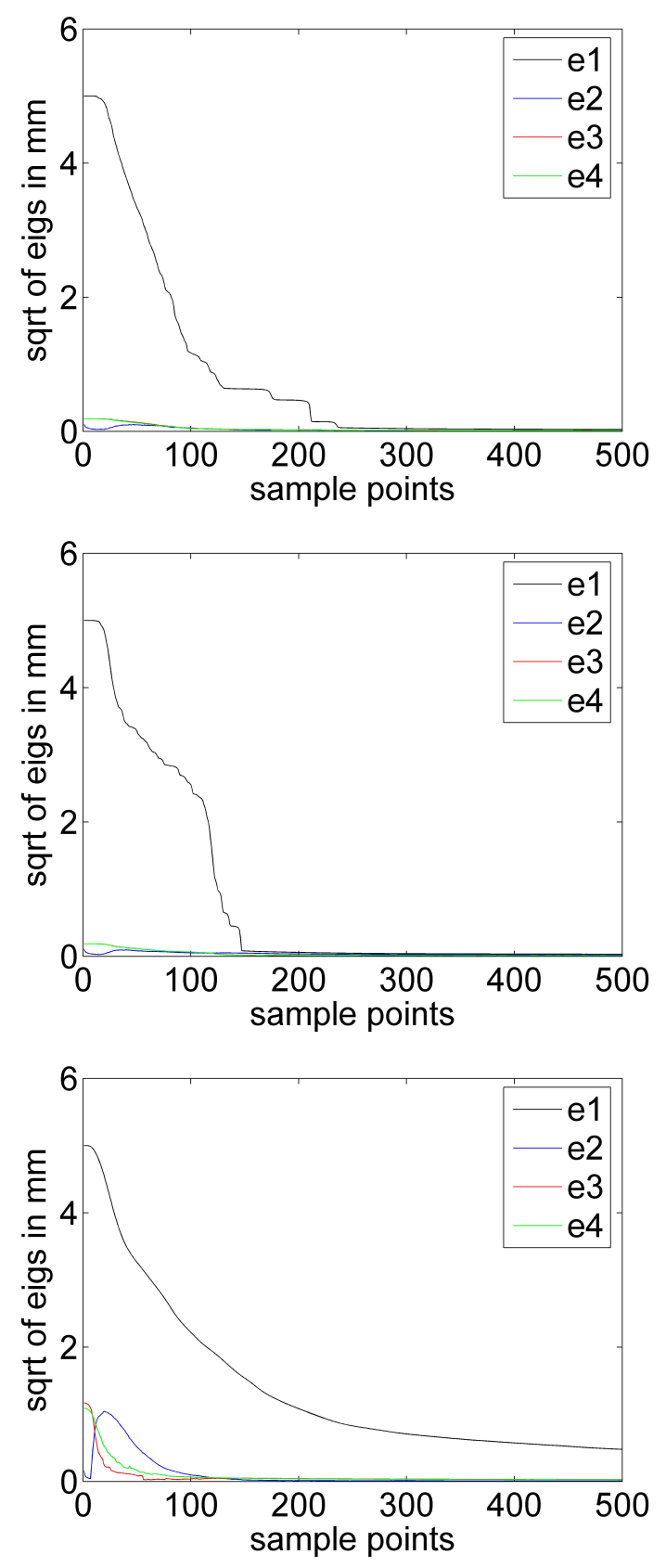

Figure 4.8: Square root of the eigenvalues e1, e2, e3, and e4 computed by my calibration filter as a function of sampled points over all trials for the three calibration data sets: calibration in which probe samples (top) much of the sphere, (middle) primarily in the $x$ and $z$ directions, and (bottom) primarily in the $y$ and $z$ directions. 
described in Appendix B). Each least-squares estimate had the bootstrap estimated covariance matrix

$$
\hat{C}_{\theta}=\frac{1}{B-1} \sum_{b=1}^{B}\left[\hat{\theta}^{* b}-\bar{\theta}^{*}\right]\left[\hat{\theta}^{* b}-\bar{\theta}^{*}\right]^{T} .
$$

I compared the covariance estimates of the least-squares solution with the covariance estimates produced by the calibration UKF for each of the three calibration data sets. Figures 4.9, 4.10, and 4.11 show the square roots of the eigenvalues $e 1$, $e 2, e 3$, and $e 4$ of the covariance estimates produced by both methods for calibration data sets 1 through 3 . The mean differences in the tip for the two methods for type 1 calibration were (in $\mathrm{mm}$ )

$$
\left[\begin{array}{l}
x \\
y \\
z \\
r
\end{array}\right]=\left[\begin{array}{l}
0.1 \\
0.0 \\
0.0 \\
0.1
\end{array}\right] \pm\left[\begin{array}{l}
0.1 \\
0.0 \\
0.0 \\
0.1
\end{array}\right]
$$

for type 2 calibrations

$$
\left[\begin{array}{l}
x \\
y \\
z \\
r
\end{array}\right]=\left[\begin{array}{l}
0.0 \\
0.0 \\
0.0 \\
0.0
\end{array}\right] \pm\left[\begin{array}{l}
0.0 \\
0.0 \\
0.0 \\
0.0
\end{array}\right]
$$


and for type 3 calibrations

$$
\left[\begin{array}{l}
x \\
y \\
z \\
r
\end{array}\right]=\left[\begin{array}{l}
0.1 \\
0.0 \\
0.0 \\
0.1
\end{array}\right] \pm\left[\begin{array}{l}
0.1 \\
0.0 \\
0.0 \\
0.1
\end{array}\right]
$$

The proposed calibration UKF covariances agreed with the bootstrapped least-squares covariance estimate.

\subsubsection{Discussion}

Closer examination of Figures 4.9, 4.10, and 4.11 revealed that eigenvalues $e 1$ and $e 4$ are identical. This was explained by the coupling of $x$ and $r$ in the computation because $x$ was in the direction of the probe; hence, the computations of $x$ and $r$ were the same.

Recall that, in the first calibration set, the probe sampled much of the sphere (thereby collecting points in all directions). Figure 4.9 indicates greater uncertainty in the first eigenvalue (the $x$ direction). This makes sense given that very little of the sphere was sampled in the $x$ direction in this type of calibration.

In the second calibration set, the probe was restricted to movement in the $x$ and $z$ directions. Figure 4.10 shows greater error in the second eigenvalue which corresponded to larger uncertainty in the $y$ direction.

The third calibration set restricted movement of the probe to the $y$ and $z$ directions. In Figure 4.10, the first eigenvalue is larger than the second and third, reflecting the increase in uncertainty in the $x$ direction. 

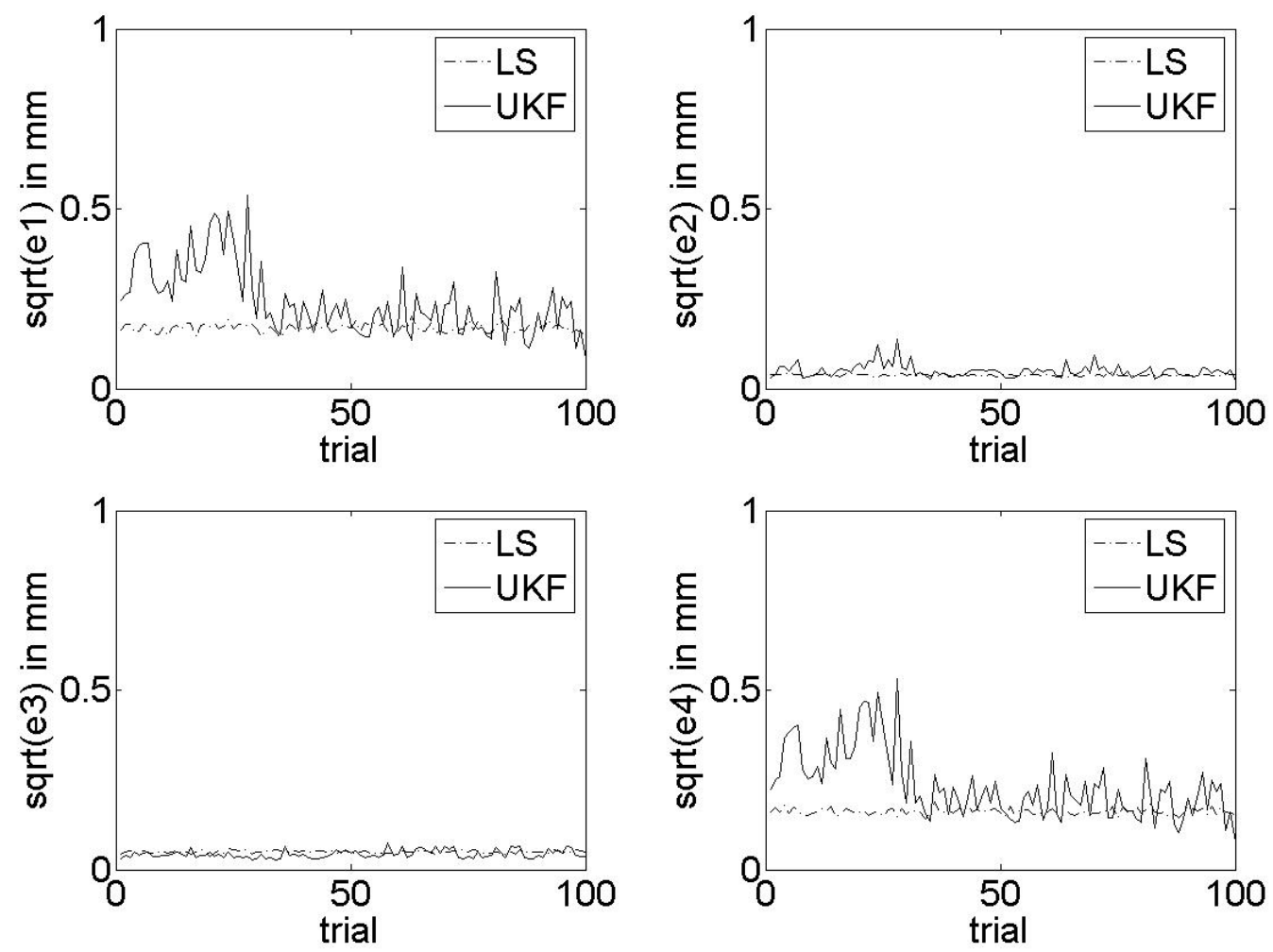

Figure 4.9: Calibration Type 1. The graphs show the square-root of the diagonal elements of the eigenvalues of the covariance matrix for 100 calibrations for the calibrations where the probe samples much of the sphere. Individual graphs show the least-squares and UKF results for the square root of the eigenvalues $e 1, e 2, e 3$, and $e 4$. The results indicated that the calibration filter estimates the covariance well, when compared with the least-squares estimate of bootstrapped covariance.

All of the 300 calibrations were collected manually. It is important to note the difficulty in collecting data in one or two directions by estimating roughly by sight.

The UKF calibration method computed covariance as input was collected from the tracking system. Therefore, calibration points could be collected until the uncertainty was sufficiently minimized. 


\subsection{A COMBINED ESTIMATE OF TRACKING AND
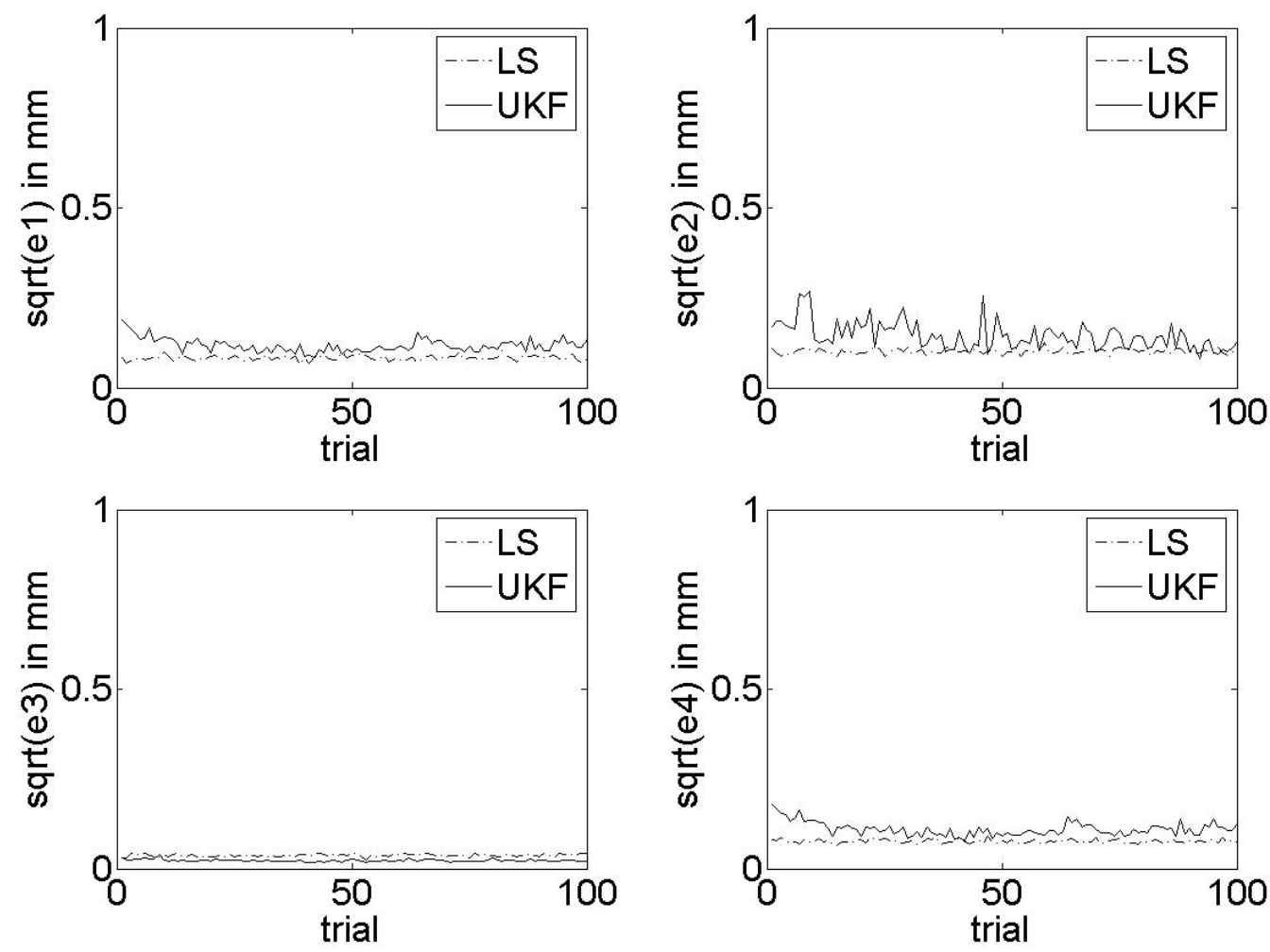

Figure 4.10: Calibration Type 2. The graphs show the square-root of the diagonal elements of the eigenvalues of the covariance matrix for 100 calibrations for the calibrations where the probe samples primarily in the $x$ and $z$ axes. Individual graphs show the least-squares and UKF results for the square root of the eigenvalues $e 1, e 2$, $e 3$, and $e 4$. The results indicated that the calibration filter estimates the covariance well, when compared with the least-squares estimate of bootstrapped covariance.

\subsection{A Combined Estimate of Tracking and Calibration Uncertainty}

In this section, I describe a UKF-based method for propagating tracking uncertainty to calibration. There are two Kalman filter extensions that enable simultaneous estimation of parameters and states: joint filtering and dual filtering [107]. In joint filtering, the parameter vector is appended to the state vector. The augmented covariance is propagated as one large matrix. Dual filtering interlaces two separate 


\subsection{A COMBINED ESTIMATE OF TRACKING AND
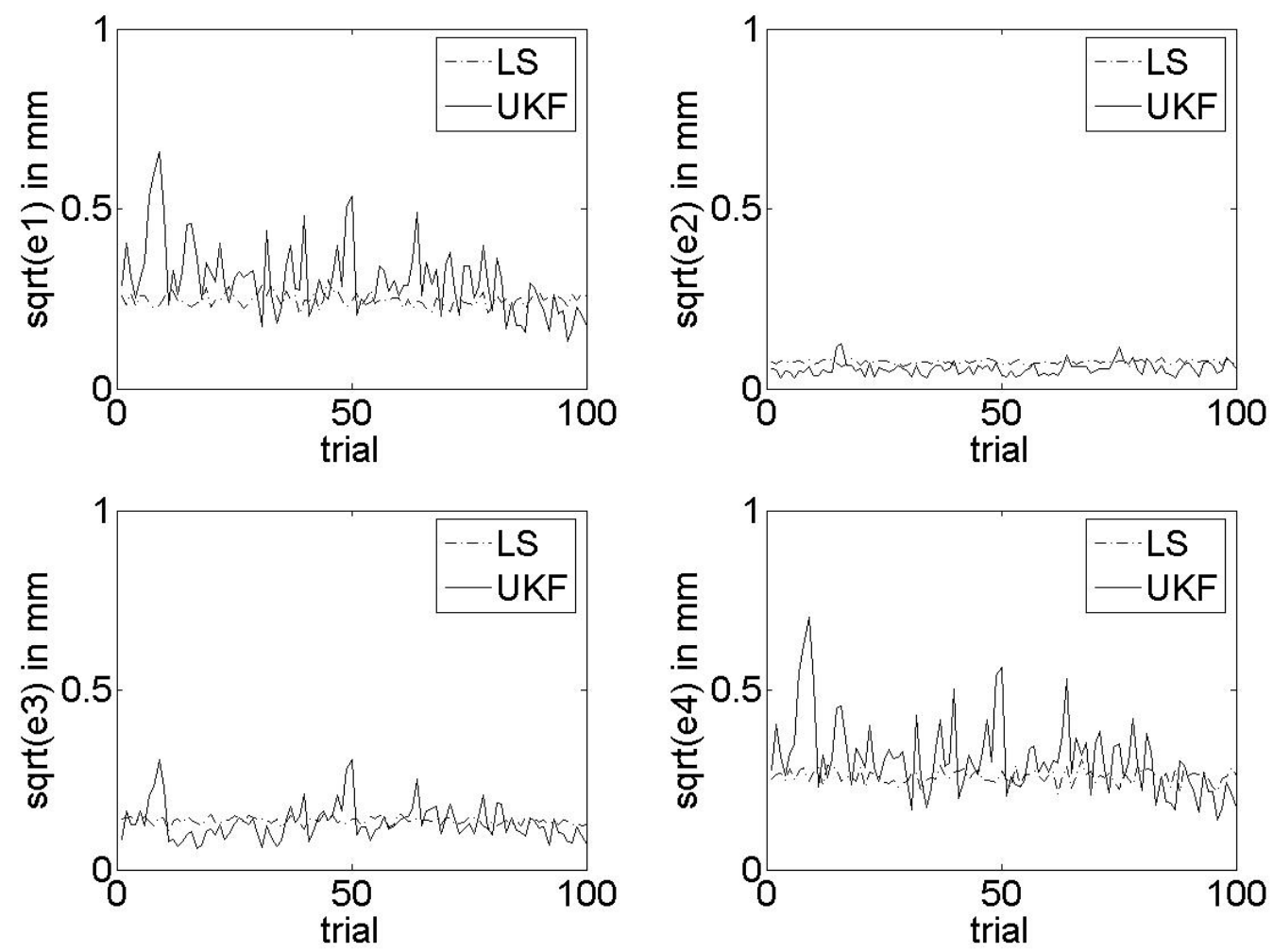

Figure 4.11: Calibration Type 3. The graphs show the square-root of the diagonal elements of the eigenvalues of the covariance matrix for 100 calibrations for the calibrations where the probe was restricted to movements in $y$ and $z$. Individual graphs show the least-squares and UKF results for the square root of the eigenvalues $e 1, e 2$, $e 3$, and $e 4$. The results indicated that the calibration filter estimates the covariance well, when compared with the least-squares estimate of bootstrapped covariance.

filters, one that estimates the state and one that estimates the parameters. The parameter and state vectors (and their associated covariances) can be decoupled as required by the application [107].

The first attempt to solve this problem used joint filtering; the tracking state vector and the calibration parameter vector were combined into a single state vector. This technique resulted in the filter diverging from a solution. According to VanDyke et al. [107], decoupling parameter and state estimation prevented poor initial estimates 


\subsection{A COMBINED ESTIMATE OF TRACKING AND CALIBRATION UNCERTAINTY}

from the parameter estimation that caused the state estimate to diverge. This section describes the dual filter for tracking and calibration.

The dual filter estimated the tracking state vector using the method described in Section 4.2 and the calibration parameter vector used the method described in Section 4.3. For each IRED measurement from the tracking system, the tracking UKF computed the pose and pose covariance of the target. The calibration UKF used the position reported by the tracking UKF as the points to be fit to the sphere. The covariance of the points, as reported by the tracking UKF, was the measurement noise for the calibration step. All process and noise models for the tracking part were the same as described in Section 4.2.

It is important to note that the UKF implementation for parameter estimation differed slightly from state estimation; hence, the tracking filter called one UKF for state estimation and the calibration portion called a different UKF implementation for parameter estimation.

\subsubsection{Experiments}

In order to test the reliability of the covariances estimated by the combined tracking and calibration filter, I needed noise-free IRED locations taken during a sphere calibration. I used the pose data from the calibration data set acquired in previous tests (see Section 4.3.1). I applied the pose to the target model to get the noise-free IRED locations. I added noise back to the noise-free IREDs drawn from a normal distribution with mean zero and covariance with diagonal entries $(0.15 \mathrm{~mm})^{2},(0.5 \mathrm{~mm})^{2}$, and $(0.15 \mathrm{~mm})^{2}$ representing position which corresponded to the noise covariance in 
the pose estimation. I ran the combined UKF and our the least-squares sphere calibration on 10000 sets of these noisy IREDs. The output of the filter was the vector of calibration parameters $\mathbf{w}=\left[x_{c}, y_{c}, z_{c}, r\right]^{T}$ of a sphere with center $x_{c}, y_{c}, z_{c}$ and radius $r$ and a covariance estimate of these parameters.

\subsubsection{Results}

Figures 4.12, 4.13, and 4.14 show the square-root of the eigenvectors of the covariance estimates produced by my tracking, calibration, and dual (combined tracking and calibration) filter methods for $e 1, e 2$, and $e 3$. Type 1,2 , and 3 calibration data sets were used in Figure 4.12, 4.13, and 4.14, respectively.

\subsection{Discussion}

One of the features that made a Kalman filter such a powerful tool for this work was that covariance estimates were computed as measurements became available from a device, rather than waiting for all of the measurements to be available. Feedback about the magnitude of the uncertainty was given immediately. Hence, the tracking data acquisition could be improved by, for example, changing the orientation of the target.

All tracking data was acquired using an Optotrak Certus, which was considered by the manufacturer to be a high accuracy system with reported maximum 3D accuracy of $0.15 \mathrm{~mm}$ [81]. I also collected many points during each of the calibrations. I demonstrated that, even under ideal conditions, uncertainty existed. Imagine the situation if a tracking system that exhibited greater error was used, such as the Aurora (Northern Digital Inc., Waterloo, Canada) system with reported RMS positional 

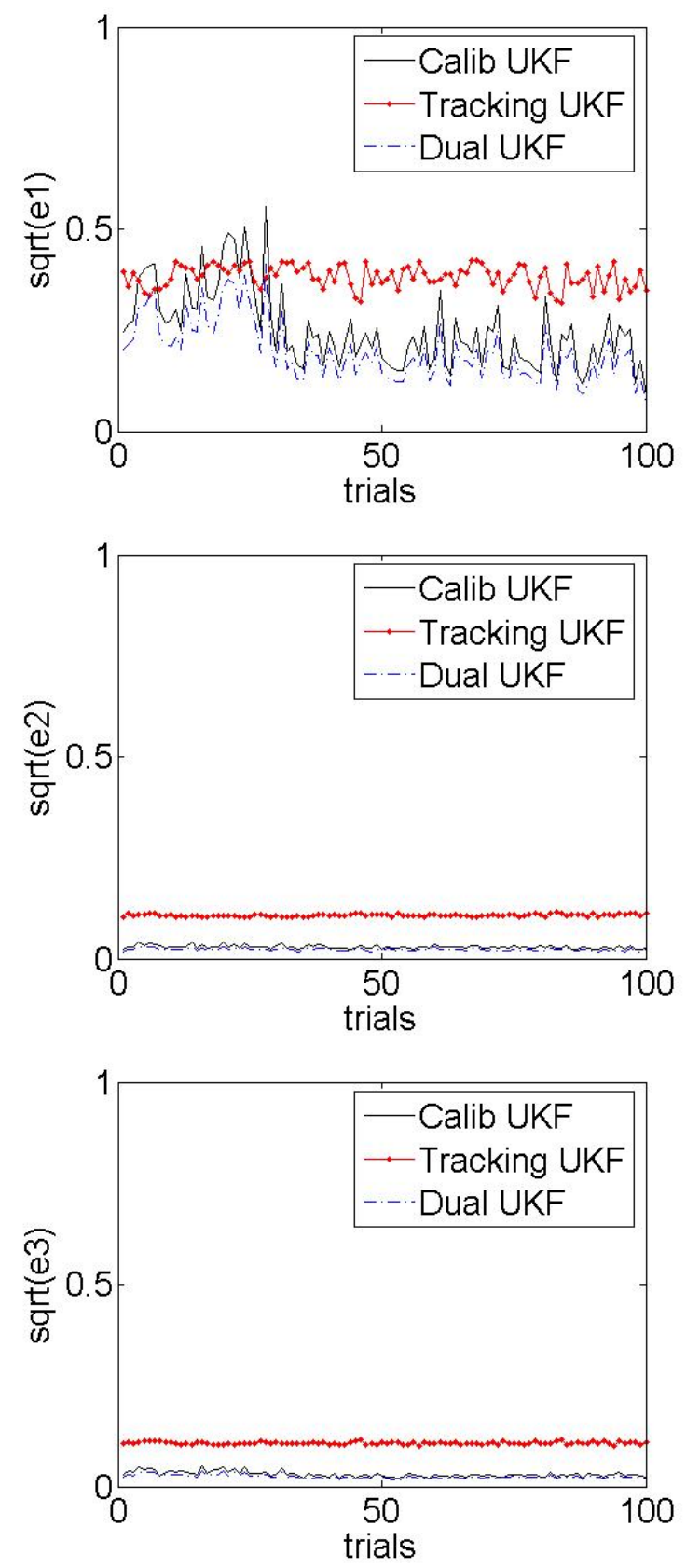

Figure 4.12: Calibration Type 1. A comparison of the calibration, tracking, and dual filter results. The graphs show the square-root of the diagonal elements of the eigenvalues of the covariance matrix for 100 calibrations where the probe sampled much of the sphere. Individual graphs show the least-squares and UKF results for the square root of the eigenvalues $e 1, e 2$, and $e 3$. 

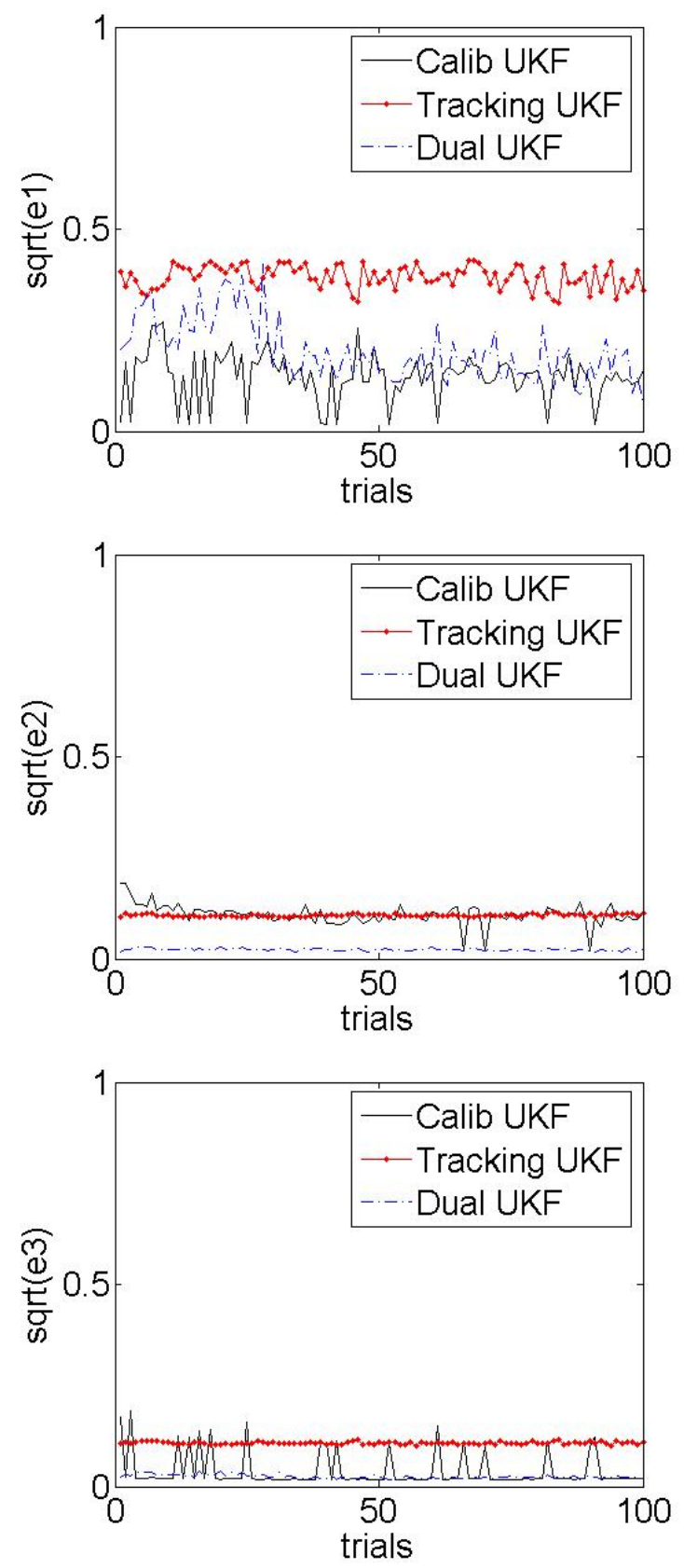

Figure 4.13: Calibration Type 2. A comparison of the calibration, tracking, and dual filter results. The graphs show the square-root of the diagonal elements of the eigenvalues of the covariance matrix for 100 calibrations where the probe samples primarily in the $x$ and $z$ axes. Individual graphs show the least-squares and UKF results for the square root of the eigenvalues $e 1, e 2, e 3$, and $e 4$. 

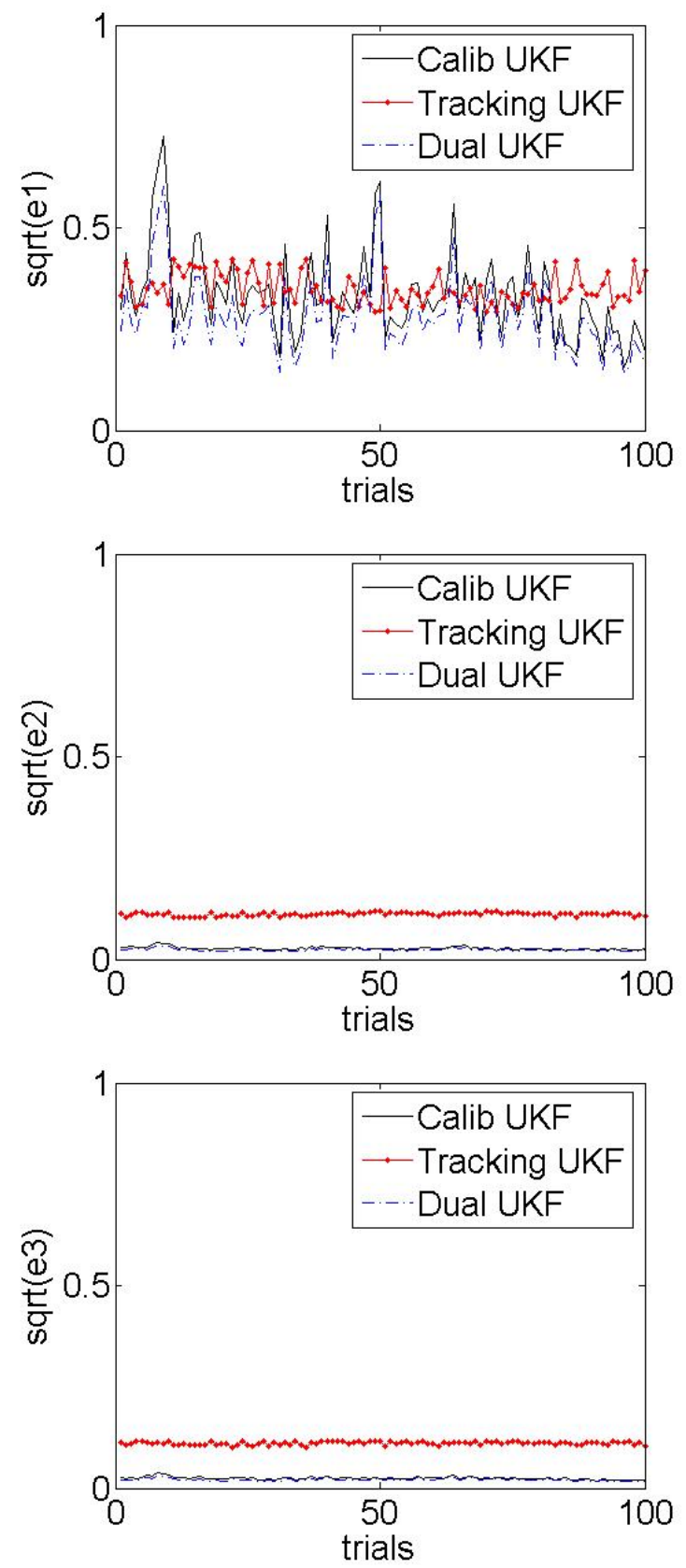

Figure 4.14: Calibration Type 3. A comparison of the calibration, tracking, and dual filter results. The graphs show the square-root of the diagonal elements of the eigenvalues of the covariance matrix for 100 calibrations where the probe was restricted to movements in $y$ and $z$. Individual graphs show the least-squares and $\mathrm{UKF}$ results for the square root of the eigenvalues $e 1, e 2, e 3$, and $e 4$. 
accuracy of $0.6 \mathrm{~mm}$ [79], or collected fewer data points in calibration.

I believe that the largest source of uncertainty in a computer-assisted surgical procedure is registration. I demonstrate in the next chapter that tracking, calibration, and registration uncertainty can be combined into an estimate of intraoperative uncertainty.

My main contribution in this chapter was to show that, in the absence of ground truth, tracking and calibration uncertainty can be reliably computed. 


\section{Chapter 5}

\section{Study: How Do Guidance and Visualization Affect a Surgeon's Performance?}

The lack of uncertainty information in navigation systems for computer-assisted surgery make it impossible for surgeons to detect and compensate for measurement uncertainty. Chapter 3 demonstrated that non-expert subjects were able to locate an obscured target in fewer steps with uncertainty visualization. In this chapter, two experiments are described that study the effects of different visualizations. For pedicle screw insertion, the perception of the magnitude of uncertainty, the accuracy of screw placement, the impingement rate, and task completion speed were measured.

The goal of this work was to determine whether expert subjects (surgeons and surgical residents) could correctly interpret the size of the uncertainty that is displayed and to determine whether the uncertainty visualization hinders performance.

The main contributions of this chapter are:

- The effect of intraoperative uncertainty from tracking, registration, and calibration on a planned linear path was computed in real-time, as the surgical tool moved through space. 
- The resulting uncertainty was visualized using an ellipse method proposed by Hoff and Vincent [33] and a new method that computed multiple lines based on the uncertainty.

- The uncertainty computation and visualization techniques were incorporated into a navigation system that used two navigation approaches for pedicle screw insertion.

- The effect of uncertainty visualization technique and navigation method on performance was studied for pedicle screw insertions.

\subsection{Pedicle Screw Fixation}

Pedicle screw fixation is a commonly used technique for stabilizing the spine in patients diagnosed with scoliosis, degenerative disc disease, fracture, tumor, infection, low back pain, pseudo arthrosis, and spinal stenosis, to name a few $[44,51,73]$. Even though retrospective studies of computer-assisted pedicle screw placements question the clinical use of pedicle screws for pain management [109], pedicle screws remain the primary treatment for fracture and scoliosis.

Figure 5.1 illustrates anatomical terms for the spine. A $2 \mathrm{~mm}$ to $10 \mathrm{~mm}$ screw is inserted through the pedicle and into the vertebral body. Plates or rods are attached to screws in adjacent vertebrae to fuse two or more vertebrae. Screw malplacement can result in vascular or spinal complications ranging from infection to cerebrospinal fluid leakage causing neurologic impairment.

Figure 5.2 shows good placement of screws inside the pedicle. Note the relative size of each screw with respect to the pedicle. The screw size is chosen to maximize the coverage of the pedicle without the screw breaching the cortex. In simple terms, the 


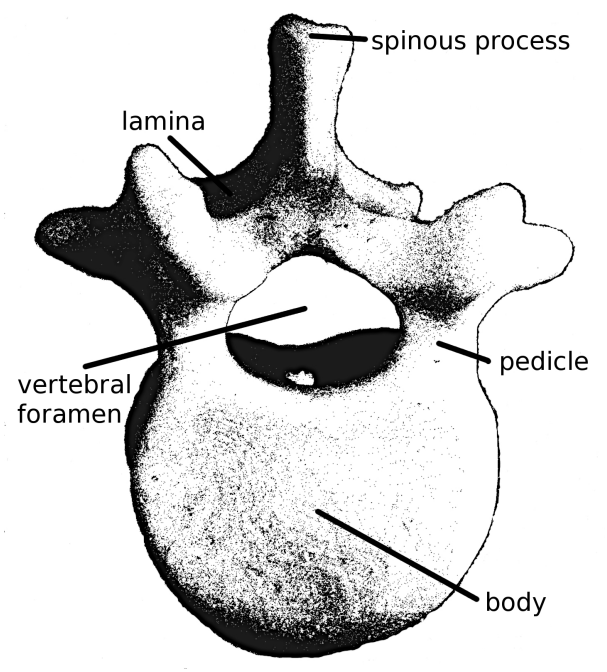

Figure 5.1: Vertebral anatomical terms.
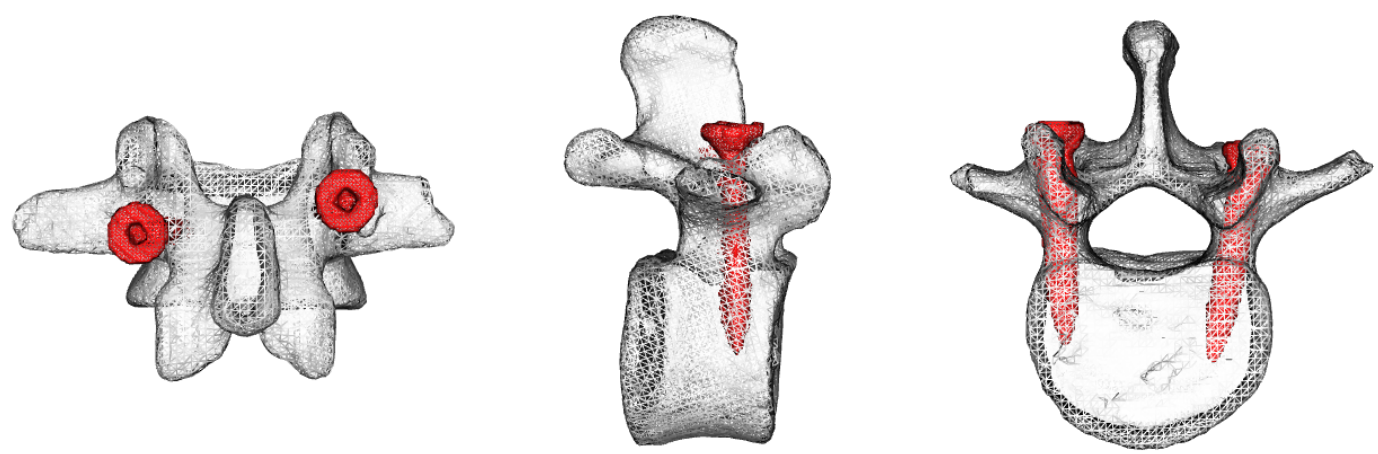

Figure 5.2: Three views of the same vertebra illustrating good placement of a pedicle screw. Screws are inserted through the lamina into the pedicle to the vertebral body.

surgeon is attempting to insert a large screw down a narrow shaft, without breaching the walls of the shaft. Breaching the walls of the shaft can have serious consequences for the patient.

Figure 5.3 shows pedicle screws from the Expedium Spine System (DePuy Spine, Inc. Raynham, Massachusetts, USA.) used clinically at Kingston General Hospital. In spinal instrumentation surgery, several vertebra (with screws inserted into both 


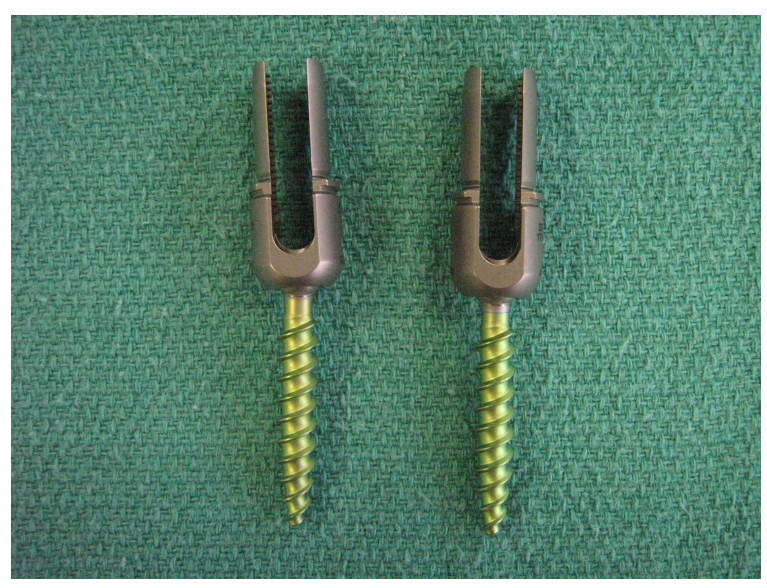

Figure 5.3: Pedicle screws from the Expedium Spine System (DePuy Spine, Inc. Raynham, Massachusetts, USA.). The screw is inserted through the pedicle. Several vertebra are fixated by attaching a metal rod or plate through the U-shaped portion of the screws.

pedicles) would be connected using a metal plate or rod which attaches through the top of the screws shown in the figure.

Thoracic pedicle screw insertions are particularly difficult because of the small diameter of the pedicles and proximity to structures at risk, such as the aorta. Translational errors of less than $1 \mathrm{~mm}$ and rotational error of less than $5^{\circ}$ are permissible [44]. Kim et al. [44] reported a screw malplacement rate of $6.2 \%$ using manual techniques, but acknowledged that other studies indicated screw malplacement rates from $3 \%$ to $44.2 \%$, with screw-related neurologic complications that ranged from $0 \%$ to $0.9 \%$. In the lumbar spine, the incidence of neurologic injury from screw malplacement ranged from $0 \%$ to $12 \%$ [106].

Image-guided surgery techniques are becoming more widely used for screw insertions. Lekovic et al. [54] reported perforation rates of $7.6 \%$ using virtual fluoroscopy 
(Fluoro-Nav, Medtronic, Minneapolis, MN, USA) and C-arm-based navigation (Siremobil Iso-C, Siemens, Munich, Germany) in the thoracic spine. Hart et al. [32] reported the occurrence of screw malplacement in the thoracic spine using imageguidance at $41 \%$ using a Stealth Station (Medtronic, Minneapolis, MN, USA) and $38 \%$ using traditional manual techniques. Lekovic et al. cited a long learning time for free-hand pedicle screw placement as a motivation for computer-assisted techniques. Some methods of percutaneous pedicle screw fixation have been reported in the literature $[26,104]$.

Kingston General Hospital has had success using the VectorVision spine navigation system (BrainLAB, Chicago, IL, USA) for pedicle screw insertion. Preoperatively, a CT scan of the target anatomy is acquired in the supine (face up) position and a 3D model of the anatomy is constructed. Intraoperatively, the patient is placed in the prone (face down) position. An incision is made and a target is fixed to the spinous process of the vertebra to be instrumented. A registration process is used to match the 3D model with the patient. The placement of the screws is planned virtually in surgery using the navigation system. Targets are attached to the surgical instruments (awls, taps, and drivers) and the instruments are calibrated. An awl is used to pierce the cortical shell of the pedicle. The pedicle probe is used to ream the cancellous bone from the pedicle and vertebral body. A tap is used to create threads inside the vertebral bone, after which a screwdriver is used to drive the screw through the path created by the tapping tool.

Figure 5.4 depicts the system in use clinically. In the figure, the top right quadrant of the interface is the most interesting. The green surgical tool is aligned with the yellow planned path. It is easy to see that if there are any errors in computing the 


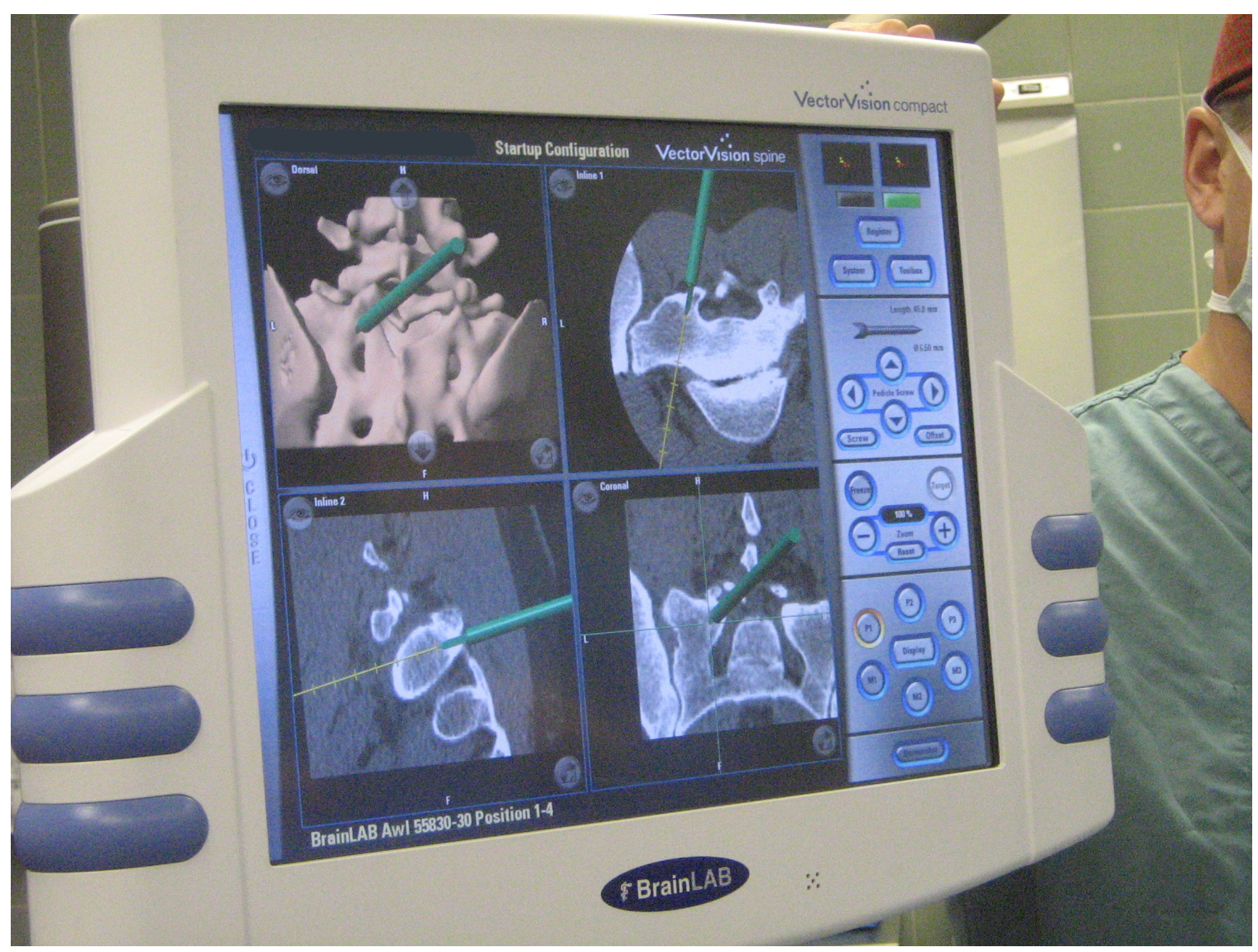

Figure 5.4: The VectorVision spine navigation system developed by BrainLAB in use in the operating room at Kingston General Hospital. The patient is undergoing a pedicle screw insertion. The top left quadrant of the interface depicts a 3D model of the patient generated from the preoperative CT scan. The other quadrants show three orthographic slices of a resampled CT volume. The surgeon's tool is shown in green. The yellow line is the planned path. The surgeon is attempting to follow the planned path with their tool.

actual location of the tool, the virtual tool may appear to be positioned properly when in fact the actual tool may be touching structures at risk. 


\subsection{Methodology}

This section describes a user study of the effect of navigation method and uncertainty visualization type on performance in pedicle screw insertions.

\subsubsection{Uncertainty Sources}

This section describes the model of camera uncertainty, the computation of the uncertainty in the target, the transformation of the camera uncertainty from the target to the tool tip, and the incorporation of registration and calibration uncertainty.

\section{Camera Covariance Model}

The user study employed a Polaris optical tracking system (Northern Digital Inc., Waterloo, Canada) with passive targets. In order to correctly model camera uncertainty, the noise model was established for the Polaris with passive markers by experiment. Because the Polaris did not report raw IRED locations, a Polaris Spectra was used to determine the noise model. The Polaris Spectra used the same camera technology as its predecessor, the Polaris, with the added feature that it returned raw IRED information.

A large target was manufactured by attaching 30 passive targets to a board. The large target was mounted on a precision optical bench. The target was oriented directly facing the Polaris Spectra, perpendicular to the viewing direction of the camera system, in the camera's z plane. The target was rotated about its axis at $10^{\circ}$ increments from $0^{\circ}$ to $60^{\circ}$. At each angle of rotation, 40 data samples were collected. This procedure was repeated at 21 locations in the measurement volume. All raw passive marker locations were collected using software provided by the manufacturer 

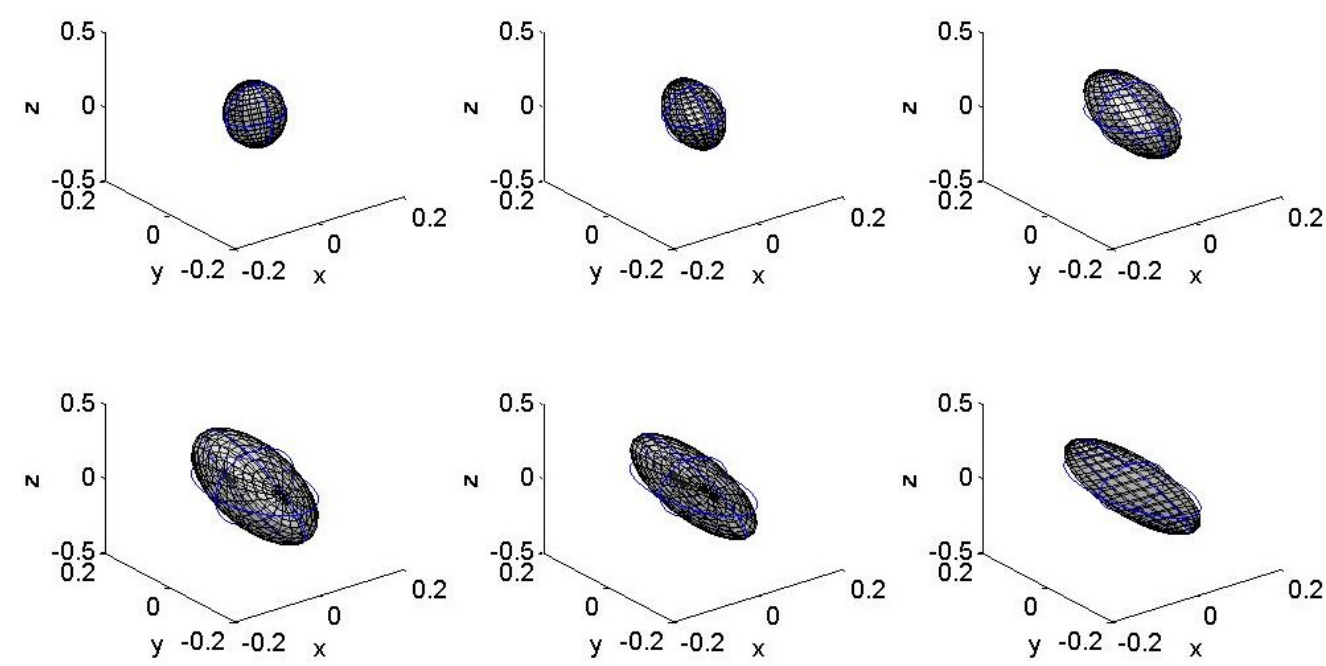

Figure 5.5: FLE computed at $10^{\circ}$ increments from $0^{\circ}$ (top left) to $60^{\circ}$ (bottom right).

of the camera system.

For each angle of rotation, 10000 bootstrapped samples of covariance were generated (see Appendix B for discussion of bootstrapping).

\section{Computation of Target Covariance}

The covariance of the target was computed using the target FLE in camera coordinates (computed in the previous section) coupled with a method described by Hoff and Vincent [33] for propagating covariance. Given a stream of $n$ data points $\mathbf{p}_{i}$ from the tracking system and the corresponding model points $\mathbf{q}_{\mathbf{i}}$ from the passive target, the covariance matrix $\mathbf{C}_{\mathbf{p}}$ described the noise distribution of each point. The covariance $\mathbf{C}_{\mathbf{p}}$ corresponded to the target FLE computed in the previous section. Let $\mathbf{p}_{i}=\mathbf{f}\left(\mathbf{q}_{\mathbf{i}}, \mathbf{x}\right)$ be a function that transforms the target model points $\mathbf{q}_{\mathbf{i}}$ for pose $\mathbf{x}$. 
The covariance matrix of $\mathbf{x}$ is [33]:

$$
\mathbf{C}_{\mathbf{x}}=\left(\mathbf{M}^{T} \mathbf{M}\right)^{-1} \mathbf{M}^{T}\left(\begin{array}{ccc}
\mathbf{C}_{\mathbf{p}} & \cdots & 0 \\
\vdots & \ddots & \vdots \\
0 & \cdots & \mathbf{C}_{\mathbf{p}}
\end{array}\right)\left(\left(\mathbf{M}^{T} \mathbf{M}\right)^{-1} \mathbf{M}^{T}\right)^{T}
$$

where $\mathbf{M}$ is the Jacobian of $\mathbf{f}$ evaluated at $\left(\mathbf{q}_{\mathbf{i}}, \mathbf{x}\right)$.

\section{Computation of Registration and Calibration Covariances}

The estimates of registration uncertainty were extracted from a laboratory study of surface-based registration for pedicle screw insertion previously reported by Queen's University [58]. The estimates were drawn from a normal distribution with total rotational mean $\mu=2.58^{\circ}\left(\sigma=1.53^{\circ}\right)$ and total translational mean $\mu=2.00 \mathrm{~mm}$ $(\sigma=1.00 \mathrm{~mm})$. The covariance estimate was the first standard deviation from the mean.

The calibration uncertainty was estimated using the unscented Kalman filter method described in Chapter 4.

\section{Transformation of Covariance}

Hoff and Vincent's [33] method for transforming a covariance matrix to a different coordinate frame was used. The purpose of this was to show the covariance estimate in CT coordinates (the coordinate system of the patient model).

The pose $\mathbf{x}$ with covariance $\mathbf{C}_{\mathbf{x}}$ was transformed by applying the transformation pose $\mathbf{w}$ to create the new pose $\mathbf{y}$ with covariance $\mathbf{C}_{\mathbf{y}}$. Let $\mathbf{y}=\mathbf{g}(\mathbf{x}, \mathbf{w})$, then the 
covariance matrix $\mathbf{C}_{\mathbf{y}}$ was computed:

$$
\mathbf{C}_{\mathbf{y}}=\mathbf{J C}_{\mathbf{x}} \mathbf{J}^{T}
$$

where $\mathbf{J}=\partial \mathbf{g} / \partial \mathbf{x}$ was the Jacobian of $\mathbf{g}$ with respect to $\mathbf{x}$ [33].

Similarly, $\mathbf{C}_{\mathbf{y}}$ was computed by assuming that the transformation $\mathbf{w}$ had covariance matrix $\mathbf{C}_{\mathbf{w}}$. The pose $\mathbf{x}$ with covariance matrix $\mathbf{C}_{\mathbf{x}}$ was transformed by vector $\mathbf{w}$ with covariance $\mathbf{C}_{\mathbf{w}}$ to get the new pose $\mathbf{y}$. The covariance matrix $\mathbf{C}_{\mathbf{y}}$ was computed as:

$$
\mathbf{C}_{\mathbf{y}}=\mathbf{J}_{\mathbf{x}} \mathbf{C}_{\mathbf{x}} \mathbf{J}_{\mathbf{x}}^{T}+\mathbf{J}_{\mathbf{w}} \mathbf{C}_{\mathbf{w}} \mathbf{J}_{\mathbf{w}}^{T}
$$

where $\mathbf{J}_{\mathbf{x}}=\partial \mathbf{g} / \partial \mathbf{x}$ and $\mathbf{J}_{\mathbf{w}}=\partial \mathbf{g} / \partial \mathbf{w}[33]$.

The covariance of the tool pose in camera coordinates, $\mathbf{C}_{\mathbf{P}}^{\mathbf{T}}$, computed using Equation 5.1, was transformed to the covariance of the probe tip in CT coordinates, $\mathbf{C}_{\mathbf{C}}^{\mathbf{S}}$, using this method. More specifically, for the pose of the tool in camera coordinates with covariance $\mathbf{C}_{\mathbf{P}}^{\mathbf{T}}$, the inverse of the pose with covariance $\left(\mathbf{C}_{\mathbf{P}}^{\mathbf{T}}\right)^{-1}$ was transformed to get the pose of the tool target with respect to the base target with covariance $\mathbf{C}_{\mathbf{B}}^{\mathbf{T}}$. Similarly, the pose of the tip of the probe with covariance $\mathbf{C}_{\mathbf{C}}^{\mathbf{S}}$ was computed with the pose of the tool in the base with covariance $\mathbf{C}_{\mathrm{B}}^{\mathrm{T}}$ transformed by the registration from the base to $\mathrm{CT}$ coordinates $\mathbf{C}_{\mathrm{C}}^{\mathrm{R}} \cdot \mathbf{C}_{\mathrm{B}}^{\mathrm{T}}$ was the calibration covariance estimated during the calibration procedure and $\mathbf{C}_{\mathbf{C}}^{\mathbf{R}}$ was the transformation from the base coordinates to $\mathrm{CT}$ coordinates computed during the registration step. The relationship between 
the covariance transformations can be summarized as:

$$
\begin{aligned}
& \mathrm{C}_{\mathrm{P}}^{\mathrm{T}} \Rightarrow \mathrm{C}_{\mathrm{B}}^{\mathrm{T}} \Rightarrow \mathrm{C}_{\mathrm{C}}^{\mathrm{S}} \\
& \Uparrow \quad \Uparrow \\
& \left(\mathbf{C}_{\mathbf{P}}^{\mathbf{T}}\right)^{-1} \quad \mathbf{C}_{\mathbf{C}}^{\mathbf{R}}
\end{aligned}
$$

Each of the covariance transformations was verified with Monte Carlo simulations.

\subsubsection{Navigation Methods}

A navigation system was developed specifically for this study. The system had two available navigation approaches: standard three orthogonal view and multiple coronal slice view.

\section{Orthographic View Navigation Method}

The first interaction method, shown in Figure 5.6, used the standard three orthogonal $\mathrm{CT}$ views and a 3D model view, similar to the VectorVision system shown in Figure 5.11. In the navigation system, the top-left quadrant was the 3D model of the patient generated from the preoperative CT scan. The remaining quadrants were three orthographic slices of a resampled CT volume aligned with the planned path. The top-right quadrant was the coronal slice (transects body into front and back) through the volume. The bottom-right quadrant was the axial slice (transects the body into top and bottom) and the bottom left is the sagittal slice (transects the body into right and left). The coronal view corresponded to looking down the planned path. Left and right movement of the probe in the axial view corresponded to left and right motion with respect to the physical bone model. Left and right movement of the 


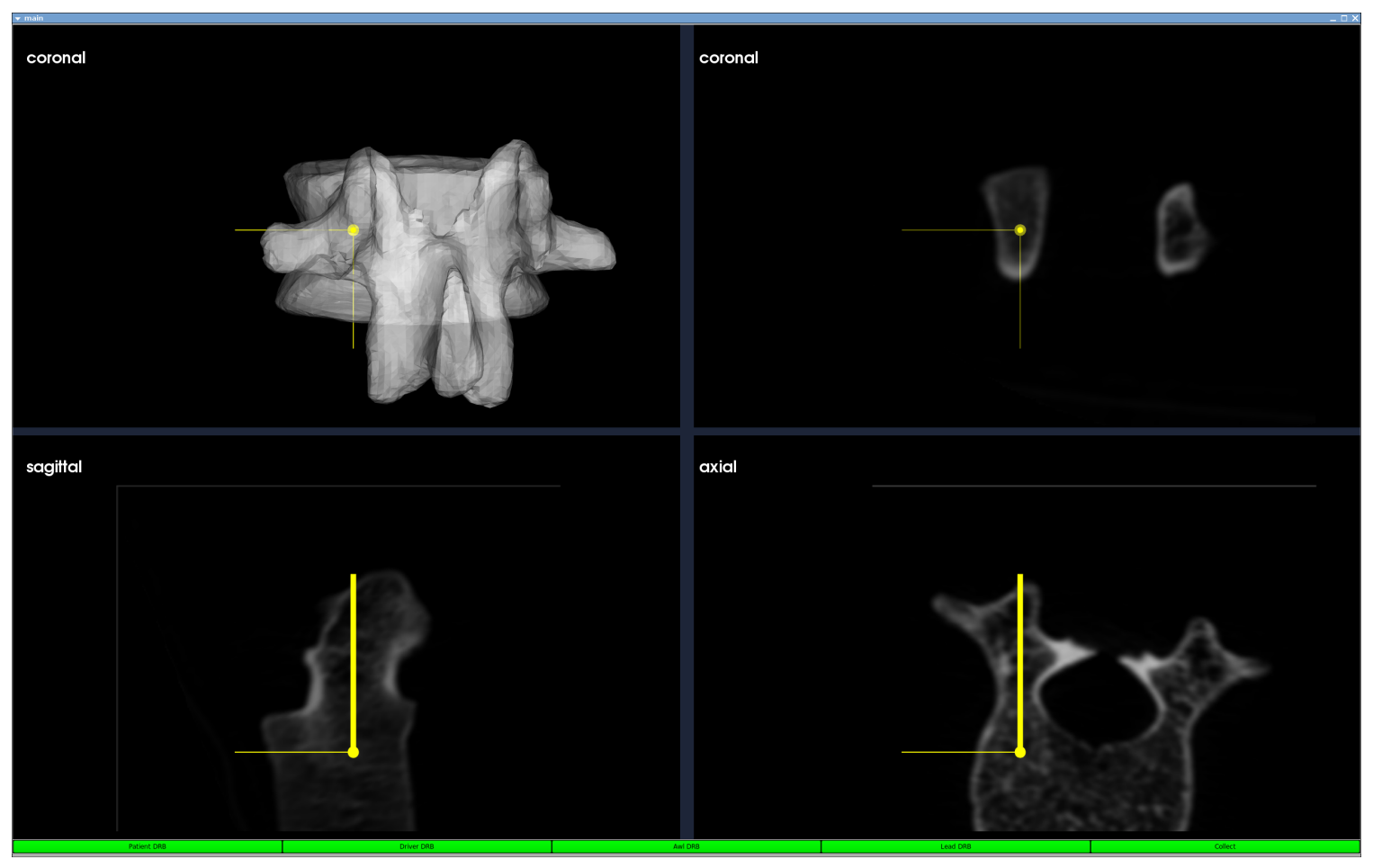

Figure 5.6: The three orthogonal view interaction method used in the system. The top left quadrant shows the 3D patient-specific model. The remaining quadrants show coronal, axial, and sagittal slices taken from a resampled CT volume and aligned with the planned path in yellow. The green buttons indicate that all of the surgical instruments are currently visible to the tracking system.

probe in the sagittal view corresponded to up and down motion with respect to the model. The yellow axes corresponded to the planned path. Each view was centered on the planned path. The bottom row of buttons indicated whether the surgical instruments were visible to the tracking system; green indicated visibility, red indicated no visibility. 


\section{Multiple Coronal Slice Navigation Method}

The second interaction method, shown in Figure 5.7, used the 3D model view and three orthogonal views with eight additional coronal views through the anatomy. In the first column, the 3D model appeared in the top cell with the axial and sagittal views in the cells below. The next three columns of cells showed coronal slices at $5 \mathrm{~mm}$ intervals from the entry point of the planned path to the vertebral body. The expectation was that the surgeon would use the coronal views to align the surgical instrument, and would use the axial and sagittal views to judge the depth of the instrument.

\subsubsection{Uncertainty Visualization Methods}

In this section, a description of two methods of uncertainty visualization used by the proposed navigation system is given.

\section{Ellipse and Cone Uncertainty Visualization}

In the ellipse and cone method of uncertainty visualization, described by Hoff and Vincent [33] and others [4, 97], an ellipse represented the components of translational covariance and cones centered on an axis represented each of the rotational components of covariance. As the probe moved through space, the ellipse and cones were updated to reflect changes in the magnitude of uncertainty in the tool pose. This is considered, by me, to be the conventional method of conveying uncertainty in the pose of an instrument, simply because other authors report its use (see Hoff and Vincent [33]).

The uncertainty of the tip in $\mathrm{CT}$ coordinates, $\mathbf{C}_{\mathbf{C}}^{\mathbf{S}}$, was rendered as an ellipse 


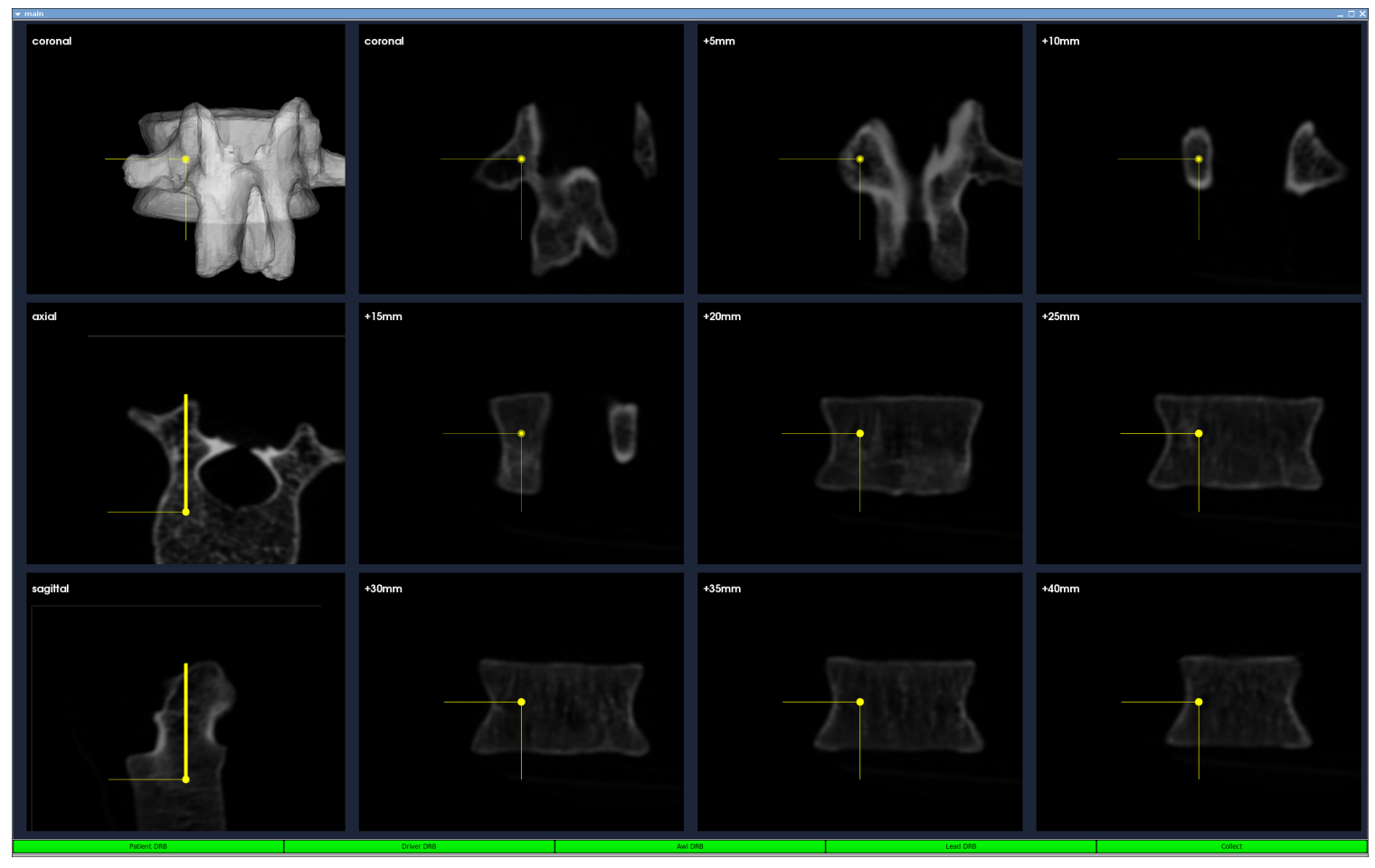

Figure 5.7: The multiple coronal slice interaction method used in the system. The first column showed the 3D model followed by the axial and sagittal views. The next three columns showed coronal slices at $5 \mathrm{~mm}$ intervals from the entry point of the planned path to the vertebral body. The green buttons indicated that all of the surgical instruments are currently visible to the tracking system.

around the tip of the probe. The major and minor axes of the ellipse were computed from the square root of the eigenvalues of the $3 \times 3$ submatrix of $\mathbf{C}_{\mathbf{x}}$ that corresponded to the translational components of the covariance. The ellipse represented approximately the $95 \%$ confidence interval.

The rotational components were visualized by a method in which the uncertainties in the $\mathrm{X}, \mathrm{Y}$, and $\mathrm{Z}$ axes were determined and rendered as cones about the axes [33] in degrees. For each axis, the orientation of the axis was computed $\mathbf{a}$ in CT coordinates using $\mathbf{a}=\mathbf{R}(\alpha, \beta, \gamma) \mathbf{e}$ where $\mathbf{R}(\alpha, \beta, \gamma)$ was the rotation matrix of the tool in $\mathrm{CT}$ 
coordinates and e was the particular unit vector in CT coordinates for each axes. Using Equation 5.2, the covariance matrix is

$$
\mathbf{C}_{\mathbf{a}}=\frac{\partial}{\partial \mathbf{e}}[\mathbf{R}(\alpha, \beta, \gamma) \mathbf{e}] \mathbf{C}_{\mathbf{e}} \frac{\partial}{\partial \mathbf{e}}[\mathbf{R}(\alpha, \beta, \gamma) \mathbf{e}]^{T}
$$

where $\mathbf{C}_{\mathbf{e}}$ is the $3 \times 3$ submatrix of rotational components of tool covariance in CT co-

ordinates and $\frac{\partial}{\partial \mathbf{e}}[\mathbf{R}(\alpha, \beta, \gamma) \mathbf{e}]$ is the Jacobian of $\mathbf{R}(\alpha, \beta, \gamma) \mathbf{e}$ with respect to $\alpha, \beta, \gamma$ [33].

Figure 5.8 depicts the ellipse and cone visualization using the three orthogonal view navigation method described in Section 5.2.2. The thick cyan tube represented the surgical tool and the thin cyan tube represented the trajectory of the surgical tool. The uncertainty in position is depicted by the magenta ellipse and the rotational uncertainty is shown by the magenta cones (seen clearly in the sagittal view of Figure 5.8) centered on the axes. In this picture, only the cones in the bottom-left quadrant can be seen. In this case, the uncertainty was small.

\section{Multi-Line Uncertainty Visualization}

The multi-line uncertainty visualization method took the current trajectory of the surgical tool and rendered a spread of several lines around that trajectory that corresponded to the covariance of the tool. That is, the covariance defined the spread of the uncertainty lines.

Figure 5.9 is a screen capture of the multi-line visualization technique with the orthogonal view navigation approach described in Section 5.2.2. In the figure, the surgical tool and surgical tool path are the thick and thin cyan tubes, respectively. Uncertainty in the path of the tool was represented in magenta. In the axial views (top quadrants) the uncertainty was rendered as circles and in the sagittal and axial views 


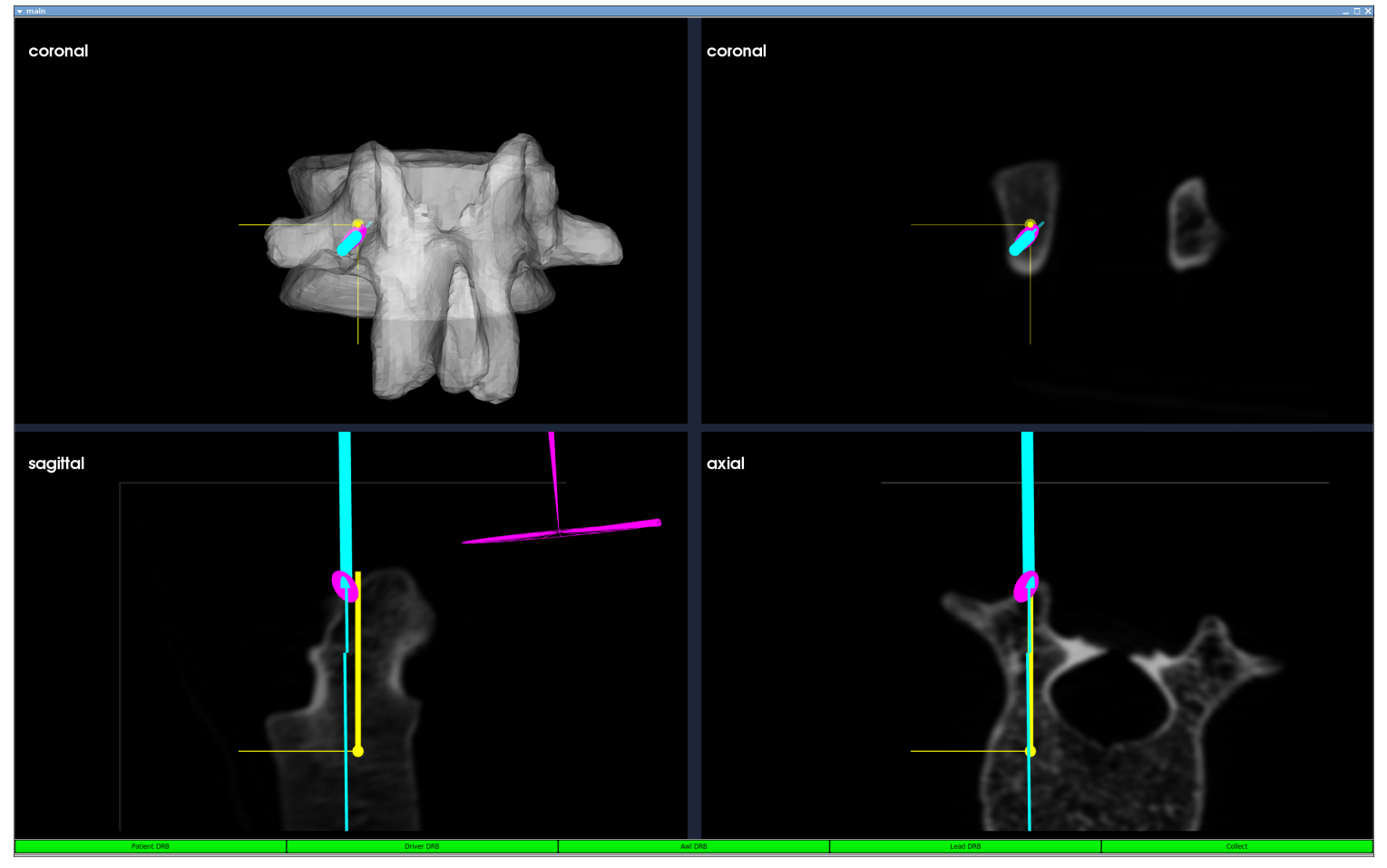

Figure 5.8: Ellipse and cone uncertainty visualization method using the three orthogonal view navigation method. The top left quadrant showed the 3D patient-specific model. The remaining quadrants showed coronal, axial, and sagittal slices taken from a resampled CT volume and aligned with the planned path in yellow. The green buttons indicated that all of the surgical instruments were then visible to the tracking system. The surgical tool and the path of the surgical tool were represented by the thick and thin cyan tubes, respectively. The magenta ellipse depicted positional uncertainty and the magenta cones (shown only in the lower-left quadrant in this picture) depicted the rotational uncertainty in degrees. The ellipse corresponded to approximately the $95 \%$ confidence interval. In this particular example, the positional and rotational uncertainty was small. 


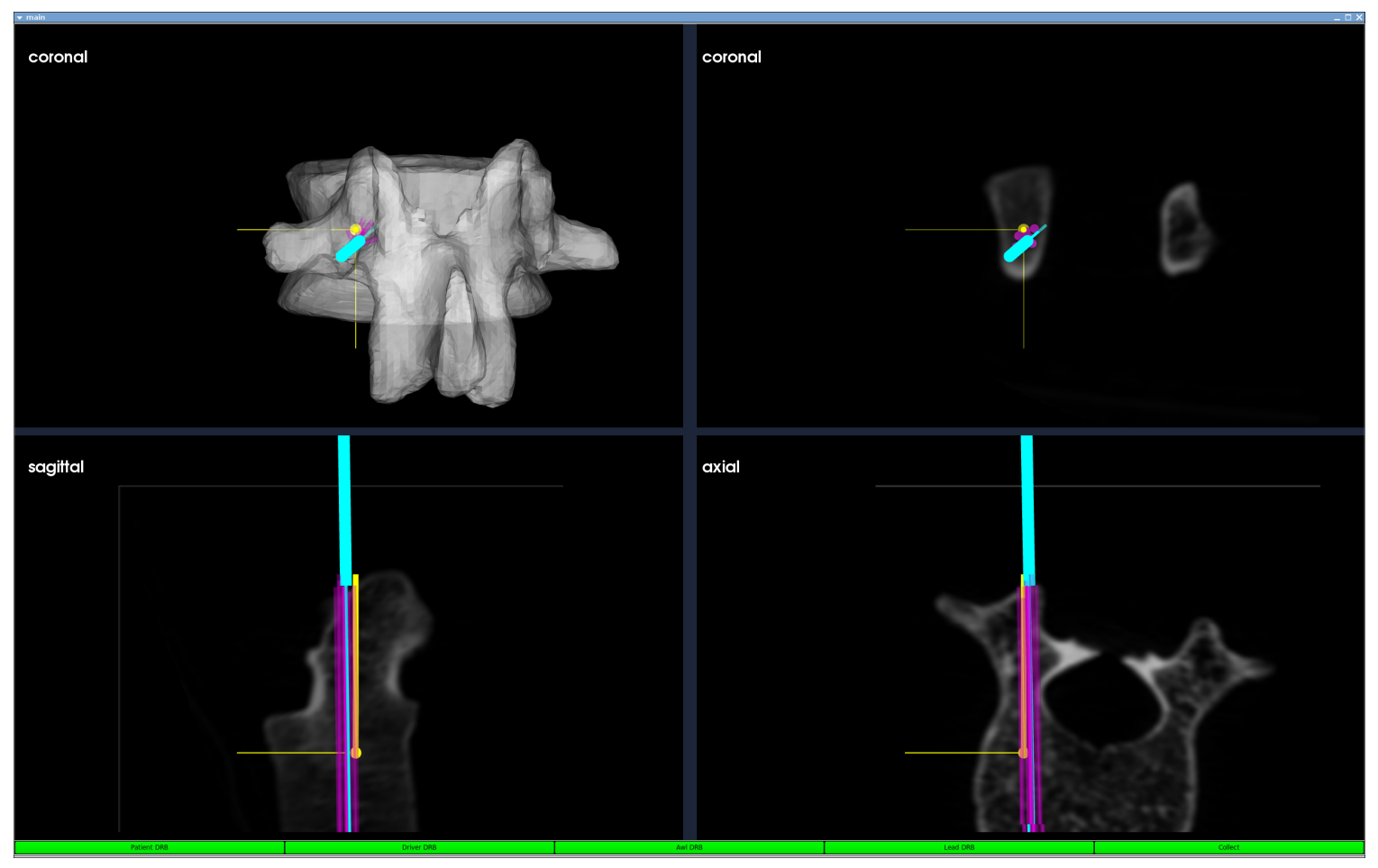

Figure 5.9: Multi-line visualization method using the three orthogonal view navigation method. The top left quadrant showed the 3D patient-specific model. The remaining quadrants showed coronal, axial, and sagittal slices taken from a resampled CT volume and aligned with the planned path in yellow. The green buttons indicated that all of the surgical instruments were then visible to the tracking system. The surgical tool and the path of the surgical tool were represented by the thick and thin cyan tubes, respectively. Uncertainty in the path of the surgical instrument was represented by the magenta lines (and circles in the coronal views) computed using the multi-line method. The possible paths of the surgical tool described the current magnitude of uncertainty. In this particular example, the positional and rotational uncertainty was small, so the lines appear dense.

(bottom quadrants) the uncertainty was rendered as lines. Figure 5.10 shows the multi-line visualization with the multiple coronal method described in Section 5.2.2.

The method used to produce lines that sampled the covariance was the unscented transform, a method proposed by Julier and Uhlmann [39]. The unscented transform is detailed in Appendix A. In short, the unscented transform takes as input a sample 


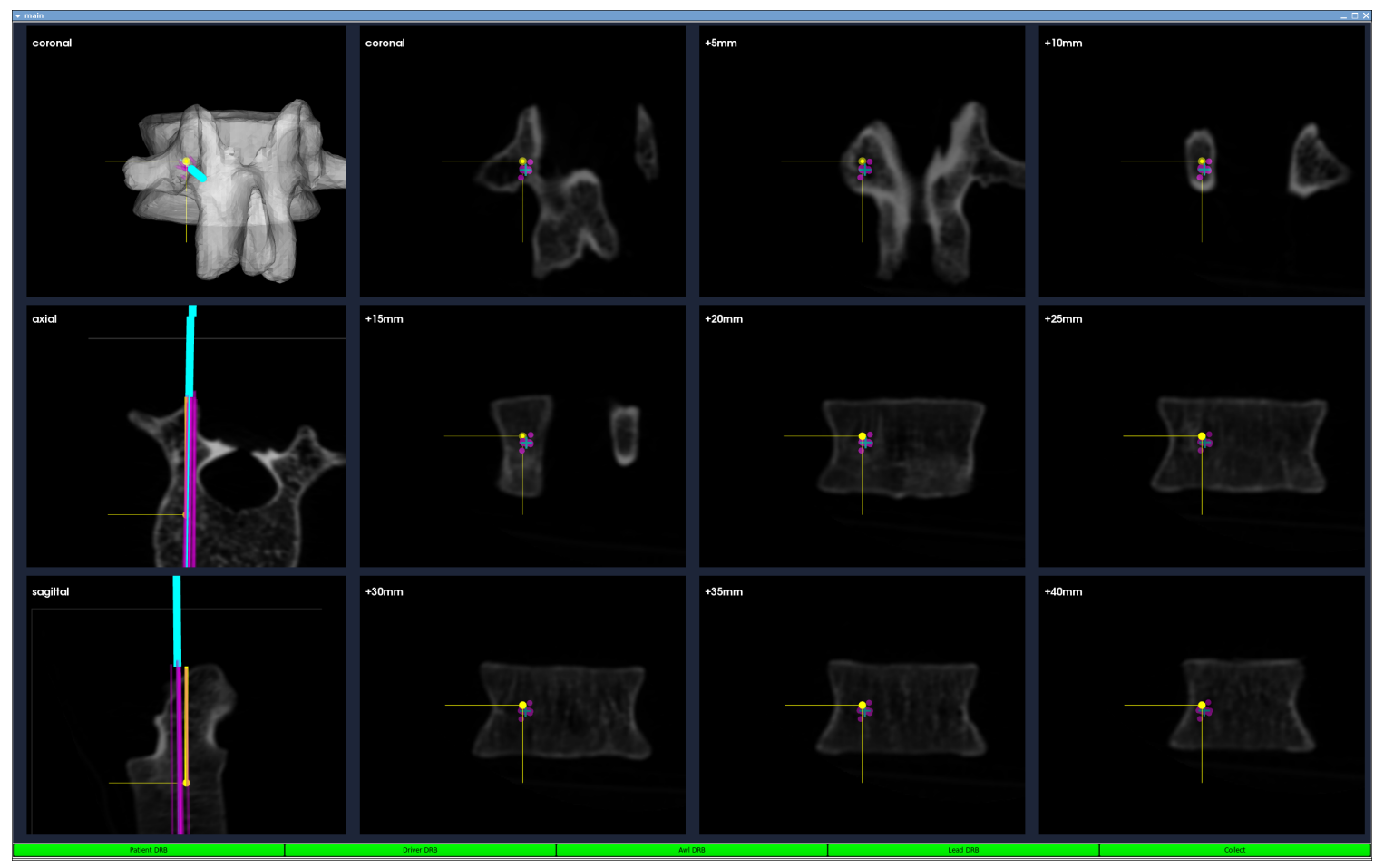

Figure 5.10: Multi-line visualization method using the multiple coronal slice view navigation method. The first column showed the 3D model followed by the axial and sagittal views. The next three columns showed coronal slices at $5 \mathrm{~mm}$ intervals from the entry point of the planned path to the vertebral body. In the model, sagittal, and axial views, the thick cyan tube represented the surgical tool and the thin tube represented the trajectory of the tool. In the coronal views, the cyan circle represented the surgical tool and the cyan cross represented the trajectory of the tool. Uncertainty in the path of the surgical instrument was represented by the magenta lines (and circles in the coronal views) computed using the multi-line method. The green buttons indicated that all of the surgical instruments were then visible to the tracking system. 
mean and covariance and produces sample points around the mean.

Given the 6 element pose $\overline{\mathbf{x}}$ and the $6 \times 6$ covariance of the pose $\mathbf{C}_{\mathbf{x}}$, the uncertainty lines were computed $\left\{\mathcal{X}=\mathcal{X}_{0}, \ldots, \mathcal{X}_{i}, \ldots, \mathcal{X}_{i+n}\right\}$. The $2 n+1$ lines were computed

$$
\begin{aligned}
\mathcal{X}_{0} & =\overline{\mathbf{x}} \\
\mathcal{X}_{i} & =\overline{\mathbf{x}}+\left(\sqrt{(n+\lambda) \mathbf{C}_{\mathbf{x}}}\right)_{i} \quad i=1, \ldots, n \\
\mathcal{X}_{i+n} & =\overline{\mathbf{x}}-\left(\sqrt{(n+\lambda) \mathbf{C}_{\mathbf{x}}}\right)_{i-n} \quad i=n+1, \ldots, 2 n
\end{aligned}
$$

where $n=6$ (the number of elements in the pose vector) and $\sqrt{ }$ denotes the Cholesky decomposition. $\lambda=\alpha^{2}(n+\kappa)-n$ is a scaling parameter, $\alpha$ is the spread of the scaling parameters around the mean $\overline{\mathbf{x}}$, and $\kappa$ is a secondary scaling parameter.

Note that each $\mathcal{X}$ calculation was the same as computing a sigma point in the unscented Kalman filter. The multi-line visualization applied each of the sigma points from the unscented transform to the pose $\overline{\mathbf{x}}$ and covariance $\mathbf{C}_{\mathbf{x}}$ of the probe. The result was 15 poses around the mean pose $\overline{\mathbf{x}}$. Each pose was applied to a line and 15 lines were rendered around the path of the probe. The intuition was that the lines showed possible trajectories given the current measured covariance. The uncertainty was updated as the probe moved through space.

\subsubsection{Subjects}

Fifteen subjects performed Experiment 1. Five attending surgeons, five junior surgical residents, three senior surgical residents, and two surgical fellows, all from the Division of Orthopaedic Surgery at Kingston General Hospital participated in the study. Thirteen subjects were male and two were female. Three subjects reported 
difficulty in distinguishing between red and green. All subjects were right-handed and only one subject reported no experience with computer-guidance displays.

Thirteen of the fifteen subjects who performed Experiment 1 also performed Experiment $2^{1}$. The two subjects that did not complete Experiment 2 were a senior resident and an attending surgeon.

Ethics approval for the experiment was obtained from the Queen's University General Research Ethics Board. All subjects signed a consent form for participation in the study (see Appendix E).

Whenever possible all subjects performed the entire study in one sitting. However, it was more common for the subjects to complete all tasks over two or three (one surgeon required six) sessions. This was because each study took over two hours to complete and it was difficult to schedule surgeons and surgical residents for extended periods of time.

\subsubsection{Apparatus}

A Polaris optical tracking system (Northern Digital Inc., Waterloo, ON) tracked the position and orientation of the tools. Three tools were used: a regular Robertson (square tipped) screwdriver, an Expedium Spine System bone awl (DePuy Spine, Inc. Raynham, Massachusetts, USA), and an Expedium Spine System bone tap (see Figure 5.11). Passive targets were affixed to each tool (see Figure 5.12). Plastic bone models were cast specifically for the study. Appendix D details this process. Mounts were manufactured for fixating the bone to a board. The mount and plastic models were manufactured such that the models could be inserted and removed from the mount with ease and precision; no intermediate registration of the physical model

\footnotetext{
${ }^{1}$ Two subjects were unable to perform Experiment 2 because of scheduling difficulties.
} 


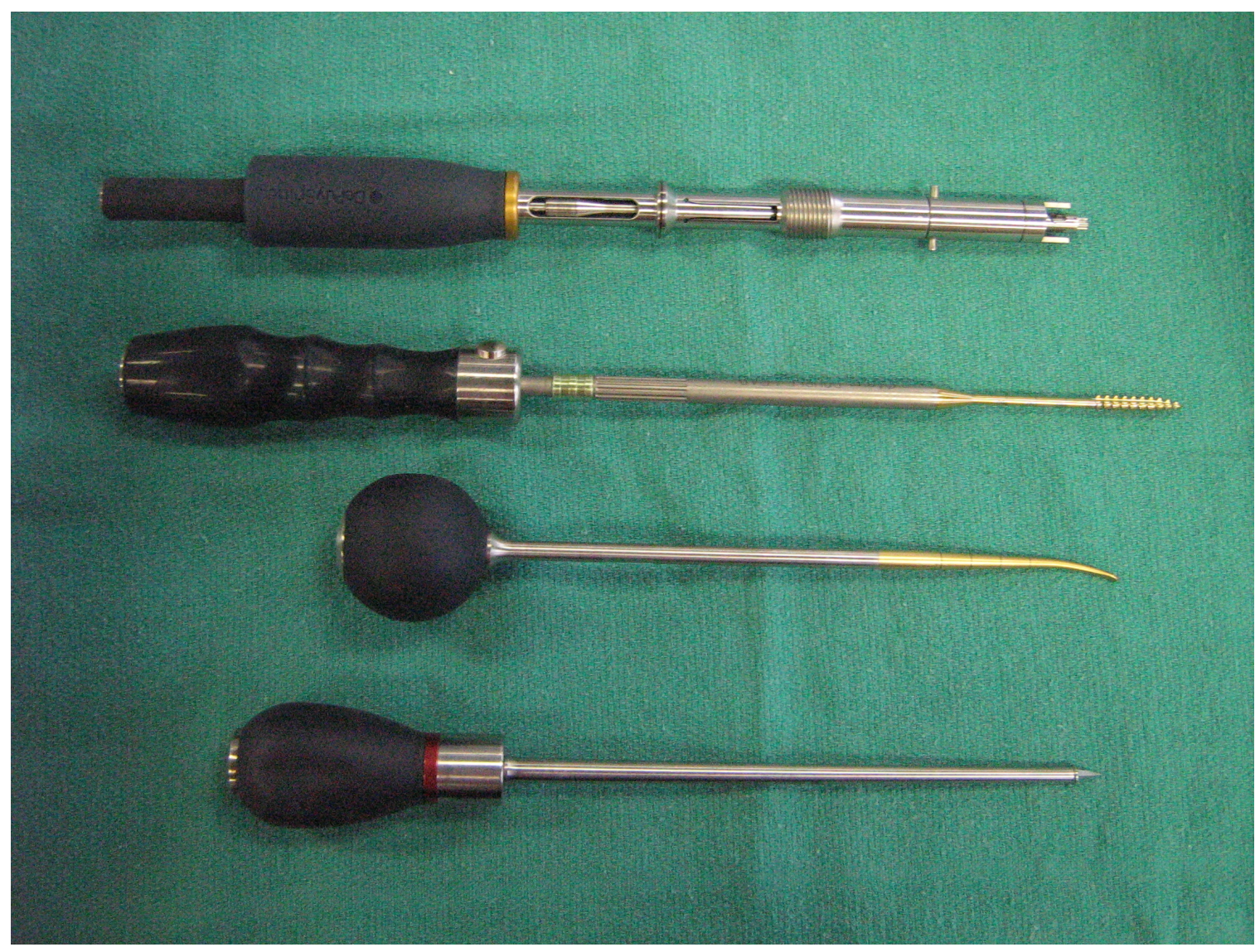

Figure 5.11: Select Expedium Spine System tools (from bottom to top) awl, pedicle probe, tap, and screwdriver. Only the awl and tap were used in the study because using actual pedicle screws is prohibitively expensive and the pedicle probe can not pierce a plastic bone model.

to the CT model was required. A fourth passive target was attached to the board for tracking the bone model. A cloth was used to mimic soft tissue, to obscure the vertebral body of the bone model from view. The apparatus is shown in Figure 5.12.

\subsubsection{Conditions}

In the experiments, subjects operated under five possible conditions. 


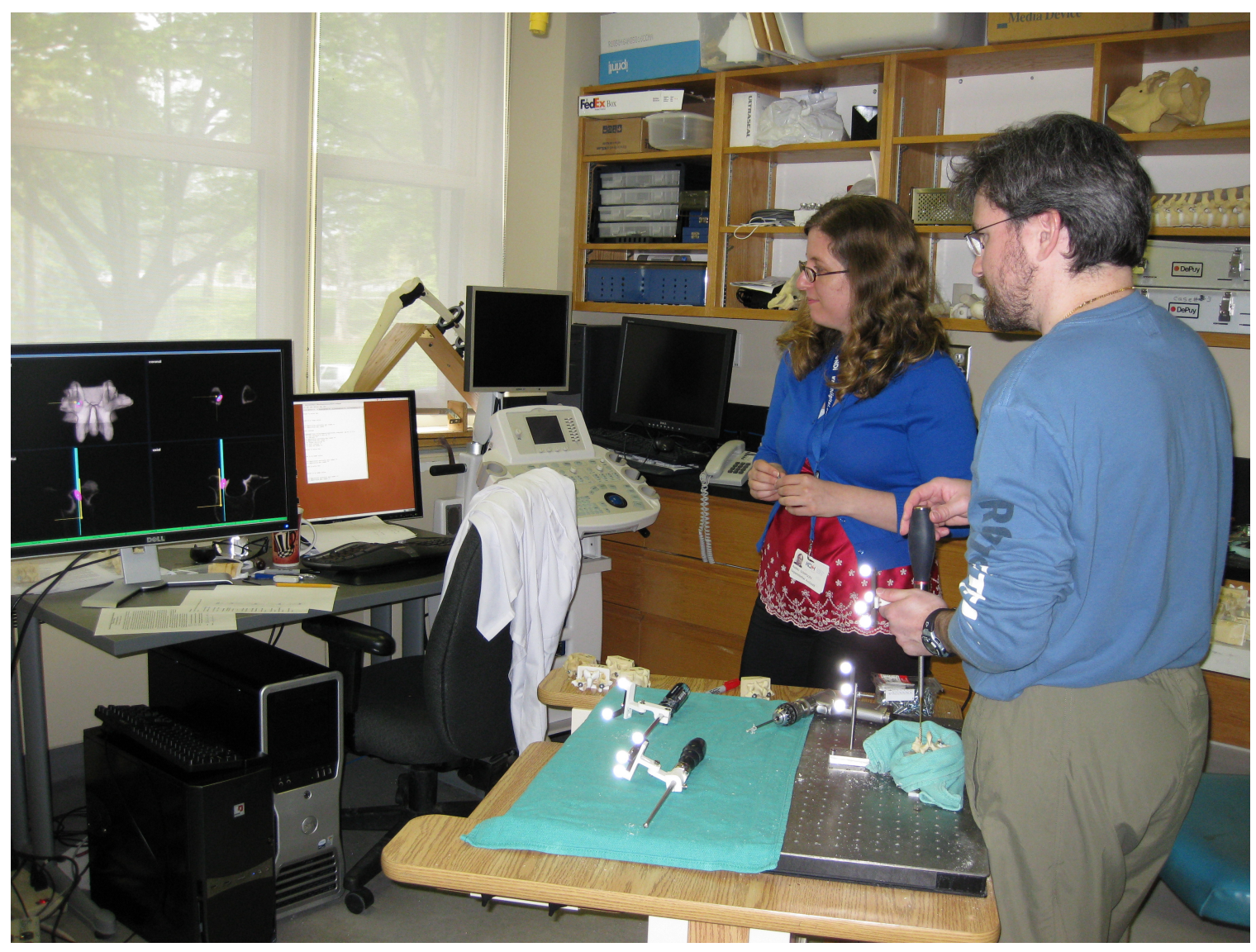

Figure 5.12: Experiment setup used in the study of pedicle screw insertions. The subject was using the navigation system to guide the tool. The optical tracking system was located just out of the camera frame, to the left.

1. No uncertainty visualization using orthographic navigation (NUO) as shown in Figure 5.6 and described in Section 5.2.2.

2. Ellipse and cones using orthographic navigation (ECO). This method (shown in Figure 5.8 and described in Section 5.2.3) showed an ellipsoid of approximately the 95th confidence interval corresponding to the tip position and cones that represented rotational uncertainty. 
3. Multi-line method of visualization using orthographic navigation (MLO) (shown in Figure 5.9 and described in Section 5.2.3). The MLO method used the multiline method to render sample poses of the tool around the mean pose according to the covariance.

4. No uncertainty visualization using multiple coronal slice navigation (NUM) as shown in Figure 5.7 and described in Section 5.2.2.

5. Multi-line visualization with multi-line rendering of uncertainty (MLM) as shown in Figure 5.10 and described in Section 5.2.3.

The ellipse and cone visualization method with the multi-view navigation was not tested to reduce the time required to complete the study.

\subsubsection{Hypotheses}

The hypotheses were:

Hypothesis 1 Accuracy of the perception of uncertainty magnitudes is better with the ellipse uncertainty visualization (ECO) than with the multi-line visualization method (MLO).

Hypothesis 2 Accuracy of the perception of uncertainty magnitudes is better with more surgical experience with either ellipse visualization (ECO) or the multi-line visualization (MLO).

Hypothesis 3 Superior and external rotation error, size of impingement, and time of screw placement is better with multi-slice navigation (NUM) than with orthographic view navigation (NUO) under the no uncertainty visualization method. 
Hypothesis 4 Superior and external rotation error, size of impingement, and time of screw placement is better in multi-slice navigation (MLM) than with orthographic view navigation (MLO) under the lines uncertainty visualization method.

Hypothesis 5 Superior and external rotation error, size of impingement, and time of screw placement is better with ellipse uncertainty visualization (ECO) than no uncertainty visualization (NUO) under orthographic navigation.

Hypothesis 6 Superior and external rotation error, size of impingement, and time of screw placement is better with the multi-line uncertainty (MLO) than no uncertainty visualization (NUO) under orthographic navigation.

Hypothesis 7 Superior and external rotation error, size of impingement, and time of screw placement is better with ellipse uncertainty visualization (ECO) than the multi-lines uncertainty visualization (MLO).

Hypothesis 8 Superior and external rotation error, size of impingement, and time of screw placement is better with the multi-line uncertainty (MLM) than no uncertainty visualization (NUM) under multi-view navigation.

Hypothesis 9 Superior and external rotation error, size of impingement, and time of screw placement is better with more surgical experience.

\subsubsection{Design}

A within-subjects design was used in this study. The study consisted of two experiments that were administered in succession. 


\section{Experiment 1: Correctly Interpreting Uncertainty Magnitudes}

Experiment 1 tested Hypothesis 1 and 2. In the first experiment, subjects were shown three instances of registration uncertainty using the ellipse and cones method with orthographic navigation (ECO). In each instance, the subjects used the navigation system to move the surgical tool to the location and orientation depicted on the computer screen. The task was to rank the magnitude of the uncertainty from largest to smallest in the three instances. Once the subject aligned the tool with the path and examined the uncertainty to their liking, the position and orientation of the tool and the covariance estimate of the tool was recorded by the navigation system. This process was repeated with nine different bones (three different registration uncertainty instances for each bone). The subject repeated this process with the multi-line method of visualization using orthographic navigation (MLO) using the same data sets.

During Experiment 1, the covariance in the tip of the tool, $\mathbf{C}_{\mathbf{C}}^{\mathbf{S}}$, was recorded for each uncertainty instance shown to the subject (recall that there were three instances in each ranking task). The ranking that the subjects assigned to the magnitude (small, medium, and large) of the uncertainty was also recorded. The magnitude of the uncertainty was computed as the trace of the principal components of $\mathbf{C}_{\mathbf{C}}^{\mathbf{S}}$. The experimenter ranked the magnitude of the uncertainty instances and scored this with respect to the ranking that the subject reported. The score was a mark out of three, which is an ordinal measurement.

An example schedule is illustrated in Table 5.1. Schedules were randomly generated prior to the experiment, ensuring that no subjects received the same schedule. The order of the stimuli were randomized. Half of the subjects were shown stimulus MLO first and the other half saw condition ECO first. Within each stimulus, the 
Table 5.1: A sample schedule of tasks for one subject for Experiment 1.

\begin{tabular}{|c|cc|}
\hline Condition & Bone & Uncertainty Instance Label \\
\hline MLO & 6 & $8,15,7$ \\
& 8 & $1,10,27$ \\
& 5 & $22,4,3$ \\
& 2 & $13,11,20$ \\
& 1 & $18,21,14$ \\
& 9 & $19,25,23$ \\
& 4 & $17,26,12$ \\
& 10 & $6,24,5$ \\
& 7 & $9,2,16$ \\
\hline ECO & 9 & $25,23,19$ \\
& 7 & $9,16,2$ \\
& 1 & $14,18,21$ \\
& 10 & $6,24,5$ \\
& 4 & $12,26,17$ \\
& 5 & $4,3,22$ \\
& 8 & $10,27,1$ \\
& 2 & $8,7,15$ \\
& 6 &
\end{tabular}

nine tasks were randomized. Note that the data displayed was the same for both conditions; only the order of the data changed. Each subject had a unique schedule in order to reduce the effects of learning or fatigue which could improve or diminish performance with later tasks. Experiment 1 took approximately 30 minutes to complete.

Note that the numeric label assigned to each registration uncertainty instance was assigned at random so the experimenter was unaware of the correct answer at the time of the experiment, that is, the experimenter could not inadvertently influence the response of the subject to the stimulus.

Experiment 1 served as training with the navigation system, prior to Experiment 2. 


\section{Experiment 2: Using Visualizations to Insert Pedicle Screws}

Experiment 2 tested Hypotheses 3 through 10. In this second experiment, the task was to insert screws into the bone models using the navigation system with NUO, ECO, MLO, NUM, and MLM conditions. Subjects performed the task eight times for each condition. An example schedule of tasks for one subject is shown in Table 5.2. In this schedule, the conditions were shown in the order: NUM, MLM, MLO, NUO, and ECO. For all subjects, the order of the conditions was randomized; however, the conditions with the same type of navigation were shown in succession. For example, NUO, ECO, and MLO were randomized as a group and NUM and MLM were randomized as a group. Half of the subjects were shown NUO, ECO, and MLO first while the other subjects were shown NUM and MLM first. The order of the bone and pedicle was randomized; however, the two pedicles for each bone were shown in sequence. The purpose of this was to reduce the number of times the bone model needed to be mounted, thereby decreasing the time required to complete the study.

Note that registration of the bone model to the 3D surface model was not computed each time a new bone was mounted. The precision fit of the bone model to the mounting device enabled the registration of one model and that transformation was applied to all copies of that model. This drastically reduced the time required to perform each task. Each time a bone was mounted, the subject verified the registration by passing the probe over the surface and visually comparing landmarks from the $3 \mathrm{D}$ 
Table 5.2: A sample schedule of tasks for one subject for Experiment 2 for all five conditions. Each entry is listed as bone, pedicle, and uncertainty registration instance label.

\begin{tabular}{|c|ccccc|}
\hline Task & NUM & MLM & MLO & NUO & ECO \\
\hline 1 & $10,2,12$ & $9,1,22$ & $7,1,7$ & $7,1,7$ & $10,2,12$ \\
2 & $10,1,15$ & $9,2,19$ & $7,2,9$ & $7,2,9$ & $10,1,15$ \\
3 & $7,2,9$ & $8,1,16$ & $8,1,16$ & $8,2,20$ & $9,1,22$ \\
4 & $7,1,7$ & $8,2,20$ & $8,2,20$ & $8,1,16$ & $9,2,19$ \\
5 & $8,2,20$ & $10,2,12$ & $9,1,22$ & $10,1,15$ & $8,2,20$ \\
6 & $8,1,16$ & $10,1,15$ & $9,2,19$ & $10,2,12$ & $8,1,16$ \\
7 & $9,2,19$ & $7,2,9$ & $10,2,12$ & $9,1,22$ & $7,1,7$ \\
8 & $9,1,22$ & $7,1,7$ & $10,1,15$ & $9,2,19$ & $7,2,9$ \\
\hline
\end{tabular}

model and the bone model ${ }^{2}$.

Each of the expert subjects inserted 40 pedicle screws. Upon completion of all tasks, the subjects were asked questions regarding their performance, visualization preferences, and information regarding handedness and color blindness. Appendix F lists the questions. Experiment 2 took approximately 100 to 160 minutes to complete.

Figure 5.13 illustrates medial and lateral breaching. In the case of a medial and lateral pedicle breach, an independent observer (a fifth year orthopaedic resident, specializing in spine surgery) measured the size of the breach from the physical bone specimen. Medial breach was measured by taking the distance at the widest point of breaching. Note that lateral breaching is not a clinical complication. In fact, a

\footnotetext{
${ }^{2}$ Initially, the concern was that the use of these bone models would be a confounding factor in the study. It turned out that because of the design of the models, if a visibly clean fit between the model and the mount was not achieved when the model was first mounted, the model was simply replaced. When the models were visually verified by passing the probe over landmarks, there were only two models that did not pass inspection (out of 260) which was because of casting irregularities (the spinous process was elongated suggesting that the model was not cured when extracted from the mold). This was supported during analysis of the results of the study. The bone models were CT scanned and the segmented scan registered to the original model (the template for all models). All of the models visually matched the template.
} 

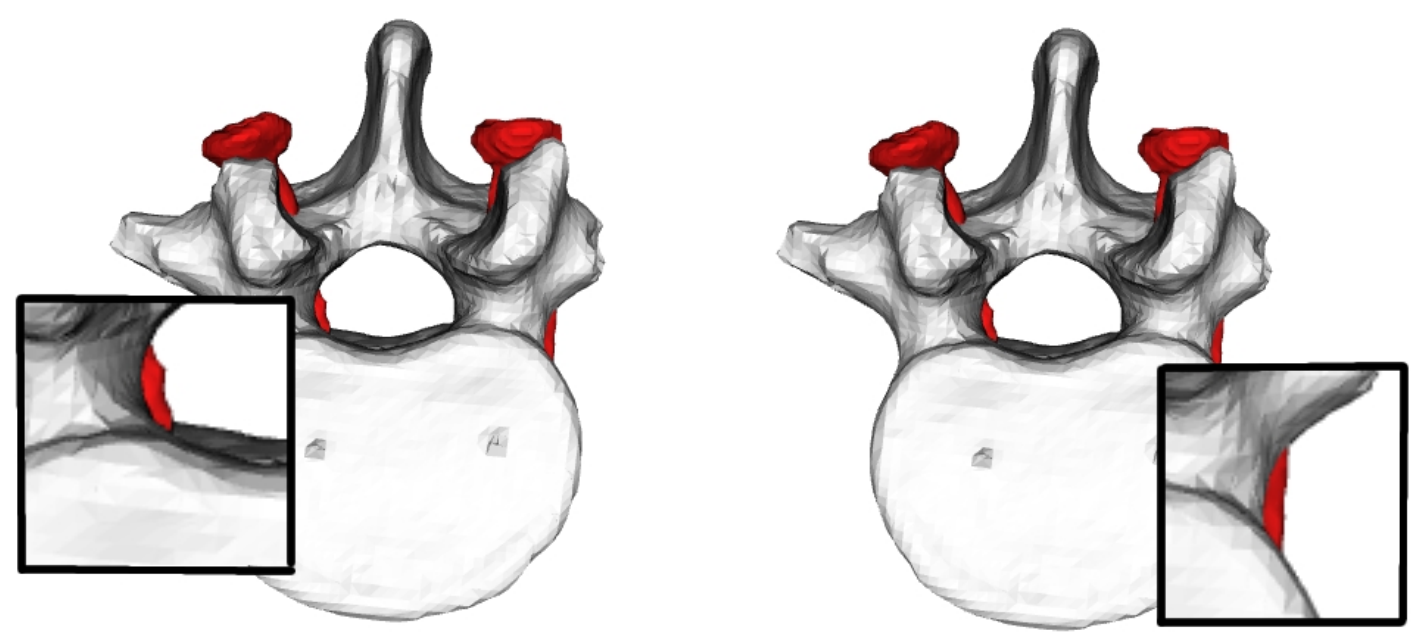

Figure 5.13: Examples of medial (left) and lateral (right) breaching of the pedicle. This impingement was measured by an independent observer. The size was taken to be the distance from the bone to the widest point of impingement.

valid alternative surgical approach is to insert the screw laterally as to avoid medial breaches of the screw into the vertebral foramen and structures at risk.

In order to quantify the discrepancy between the planned path and the final screw location, the 3D models of the bone and screws from preoperative CT scans were constructed. The post-operative model was registered to the preoperative model and planned path, and the same transformation was applied to the screws. The covariance of the screw model points was computed. The direction of a screw was determined as the direction of the eigenvector corresponding to the largest eigenvalue of the covariance matrix. The superior rotation error was measured by projecting the direction of the screw onto the $y z$ plane (note that a positive superior rotation had a negative $y$ component). The external rotation error was measured by projecting the direction of the screw onto the $x z$ plane (note that a positive external rotation 

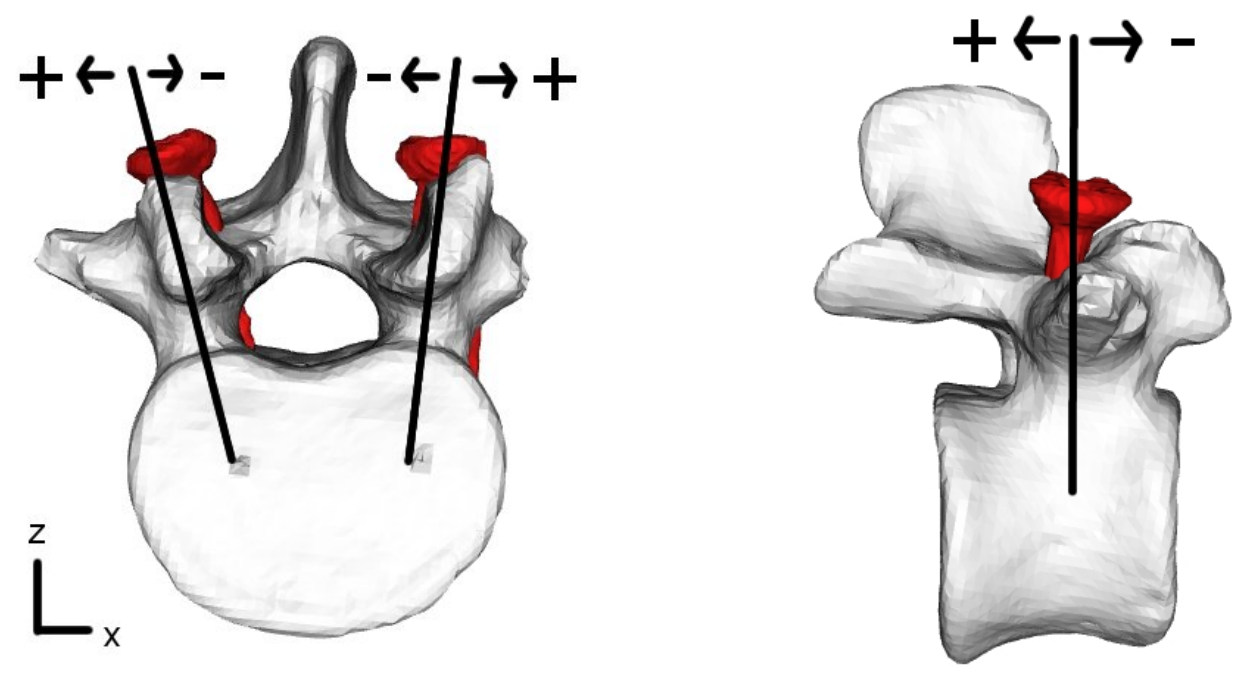

Figure 5.14: The discrepancy between the planned path and the final screw location was measured using (left) external and (right) superior rotation error measurements. The black line represents the planned path. Negative external rotation indicates lateral breaching of the pedicle and positive external rotation indicates medial breaching.

had an $x$ component greater than zero). The deviation of the post-operative screw location from the preoperative planned path was computed in this way because all of the preoperative plans were aligned with the $z$ axis. Figure 5.14 illustrates superior and external rotation error, as measured in the study.

In summary, four measurements were made for each task:

1. the superior rotation error of the final screw location from the preoperative planned path,

2. the external rotation error of the final screw location from the planned path,

3. the size of any medial breach of the pedicle (impingement), and

4. the time it took for the subject to insert one screw. 
The results of the study were analyzed using hypothesis testing wherein a null hypothesis states no effect (or no difference) between groups and the alternative hypothesis indicates the presence of an effect or a difference in groups. A research hypothesis typically predicts an effect or difference, generally a researcher expects the alternative hypothesis is supported [95].

Equivalence testing is a recent development in statistical methodology used for testing proof of similarity between groups within a small amount, $\Delta$; measures that differ by at most $\Delta$ are considered to be equivalent [92]. $\Delta$ is chosen according to the application. In the equivalence testing of this study, $\Delta=2^{\circ}$ was chosen for superior and external rotation error because rotational errors of less than $5^{\circ}$ are permissable clinically in pedicle screw insertion [44]. $\Delta=2 \mathrm{~mm}$ was chosen for the impingement tolerance because Laine et al. [51] report the 'safe zone' for medial perforation is 0 to $4 \mathrm{~mm}$ in the lumbar spine. The equivalence of multi-slice navigation and orthographic navigation with respect to superior rotation error, external rotation error, and impingement was tested.

\section{$5.3 \quad$ Results}

In this section, the results of the user study are presented.

\subsubsection{Experiment 1}

The purpose of Experiment 1 was to quantify whether subjects could correctly identify uncertainty magnitudes using the ellipse and cone uncertainty and multi-lines uncertainty methods.

The intra-rater reliability of each subject's scores was computed using Cronbach's 
alpha [9]. This gives an estimate of the consistency of the scores in the study. In this case, consistency is a measure of whether subjects' responses with respect to one visualization type were consistent with the other visualization type. The consistency test is necessary because these types of measurements have not been studied before. Table 5.3 lists Cronbach's alpha for the fifteen subjects in Experiment 1. The table indicates that twelve out of fifteen subjects had acceptably high consistency. Interestingly, there was some anecdotal evidence with respect to the three subjects who had low consistency scores. One subject commented that they stopped paying attention to the ellipses after four trials. Another suggested that their results with the first experiment may not be very good because the subject was post-call and had been working for 36 hours prior to the experiment (which was unknown to the experimenter at the time). The third subject with poor consistency reported trying to "beat the test" and "find the pattern" in the solutions (when the answers were randomized). The other twelve subjects did not report fatigue in Experiment 1.

Now that the reliability of the measurers had been established, attention was paid to hypothesis testing.

Hypothesis 1 Accuracy of the perception of uncertainty magnitudes is better with the ellipse uncertainty visualization (ECO) than with the multi-line visualization method (MLO).

Results of the Mann-Whitney test showed that subjects were significantly better at ranking uncertainty magnitudes with the ellipse uncertainty visualization than the multi-line method $(Z=-2.395, p<0.05)$.

Mean score for the ellipse uncertainty visualization was $2.8 \pm 0.1 \mathrm{SE}(92.6 \% \pm$ $1.7 \% \mathrm{SE}$ ), which as expected was well above the chance value of $17 \%$. Mean score for 
Table 5.3: Intra-rater Reliability of Measurements in Experiment 1

\begin{tabular}{|c|cc|}
\hline Subject & Cronbach's Alpha & Interpretation \\
\hline 1 & 0.78 & acceptable consistency \\
2 & 0.99 & high consistency \\
3 & 0.89 & high consistency \\
4 & 0.31 & unacceptable consistency \\
5 & 0.92 & high consistency \\
6 & 0.96 & high consistency \\
7 & 0.31 & unacceptable consistency \\
8 & 0.94 & high consistency \\
9 & 0.95 & high consistency \\
10 & 0.99 & high consistency \\
11 & 0.10 & unacceptable consistency \\
12 & 0.76 & acceptable consistency \\
13 & 0.95 & high consistency \\
14 & 0.95 & high consistency \\
15 & 0.78 & acceptable consistency \\
\hline
\end{tabular}

the multi-line uncertainty visualization was $2.6 \pm 0.1 \mathrm{SE}(87.7 \% \pm 2.0 \% \mathrm{SE})$. The results for mean score for each subject are plotted in Figure 5.15 where the mean score appears slightly higher for the ellipse uncertainty visualization method.

Hypothesis 2 Accuracy of the perception of uncertainty magnitudes is better with more surgical experience with either ellipse visualization (ECO) or the multi-line visualization (MLO).

A Kruskal-Wallis test did not show significant effects of experience on score for evaluating ellipse uncertainty $\left(\chi^{2}=0.368, p=0.832\right)$ or the multi-line $\left(\chi^{2}=0.321\right.$, $p=0.852)$ uncertainty visualization.

Mean score for the ellipse and multi-line uncertainty for subjects in the junior residents category were $2.7 \pm 0.1 \mathrm{SE}(91.1 \% \pm 3.3 \% \mathrm{SE})$ and $2.6 \pm 0.1 \mathrm{SE}(87.4 \%$ 


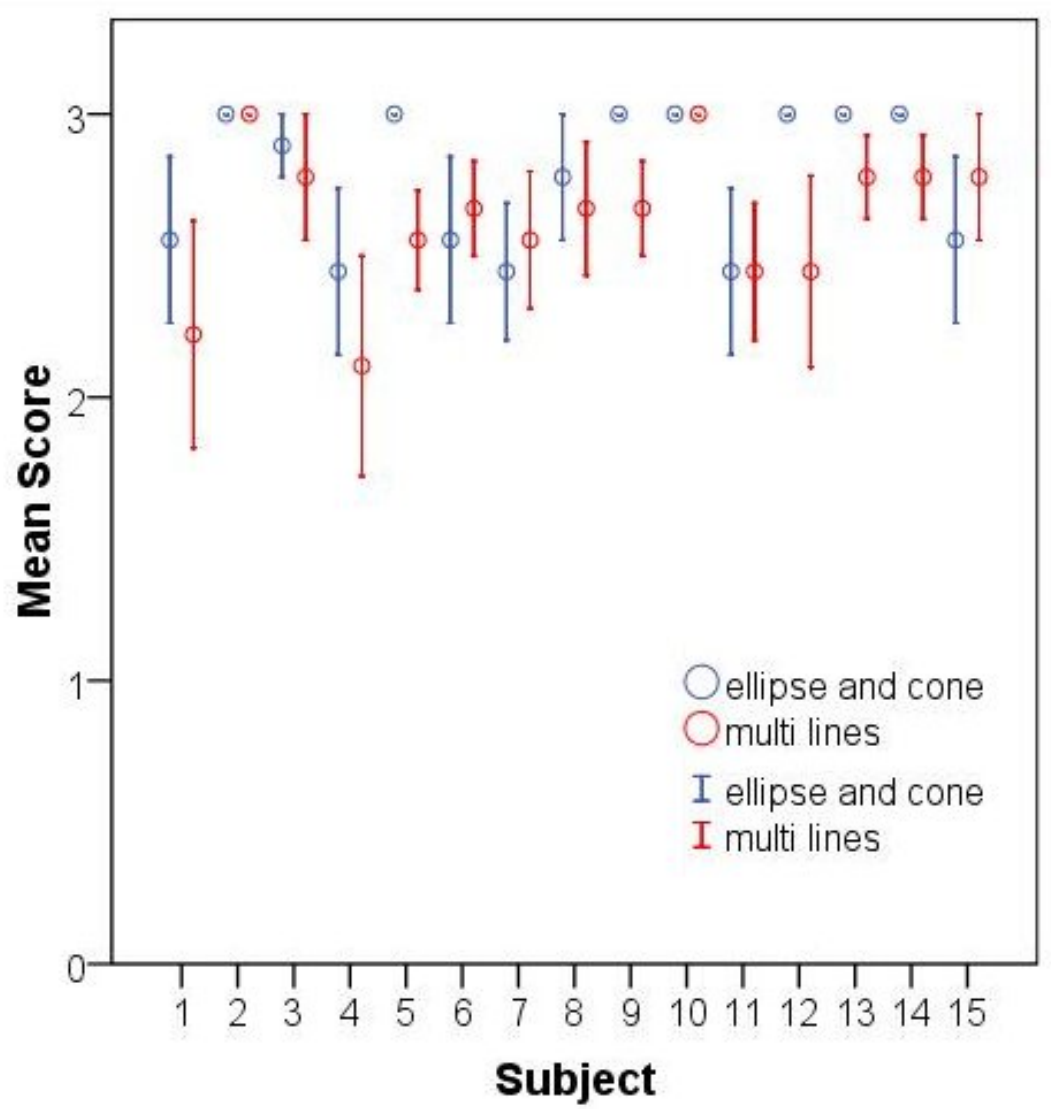

Figure 5.15: Mean ranking score for each subject by visualization method. The mean score was better for the ellipse and cone method. The mean ranking score for the ellipse and cone method was $2.8 \pm 0.1 \mathrm{SE}(92.6 \% \pm 1.7 \% \mathrm{SE})$ and for the multi-line visualization $2.6 \pm 0.1 \mathrm{SE}(87.7 \% \pm 2.0 \% \mathrm{SE})$ for the multi-line visualization.

$\pm 3.3 \% \mathrm{SE})$, respectively. The senior resident and fellows category had mean scores of $2.8 \pm 0.1 \mathrm{SE}(93.3 \% \pm 3.3 \% \mathrm{SE})$ and $2.6 \pm 0.1 \mathrm{SE}(85.9 \% \pm 3.3 \% \mathrm{SE})$ for ellipse and multi-line uncertainty, respectively. The surgical staff had mean scores of $2.8 \pm$ $0.1 \mathrm{SE}(93.3 \% \pm 3.3 \% \mathrm{SE})$ and $2.7 \pm 0.1 \mathrm{SE}(89.6 \% \pm 3.3 \% \mathrm{SE})$. The results for mean score for each experience level are plotted in Figure 5.16 where the mean score is slightly higher for the ellipse uncertainty visualization method across all experience 


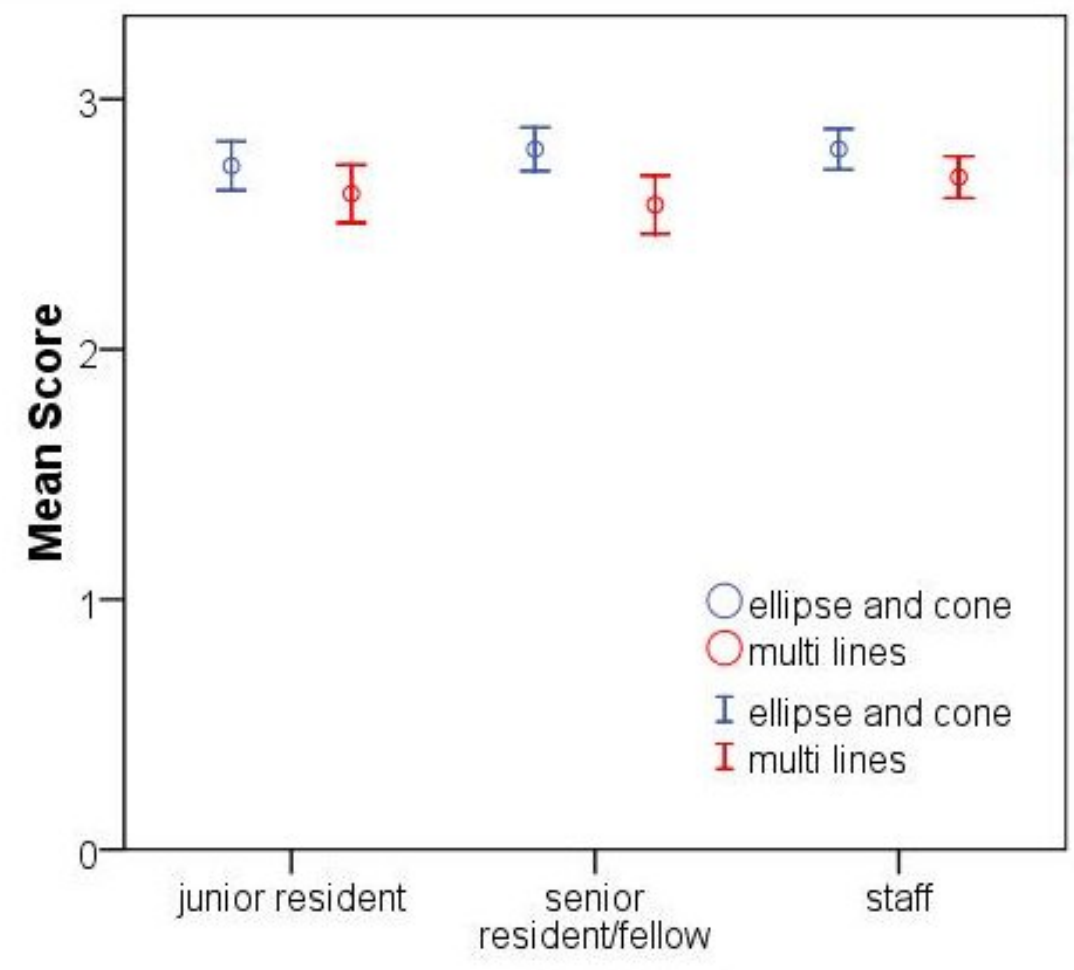

Experience

Figure 5.16: Mean score for each experience level by visualization. The mean score was similar for both the ellipse and multi-line methods $91.1 \% \pm 3.3 \% \mathrm{SE}$ and $87.4 \%$ $\pm 3.3 \% \mathrm{SE}$ for the junior group, $93.3 \% \pm 3.3 \% \mathrm{SE}$ and $85.9 \% \pm 3.3 \% \mathrm{SE}$ for the senior group, and $93.3 \% \pm 3.3 \% \mathrm{SE}$ and $89.6 \% \pm 3.3 \%$ SE for the staff group.

levels.

\subsubsection{Experiment 2}

The purpose of Experiment 2 was to assess whether the subject was able to avoid medial breaches of the pedicle and follow the planned path using the navigation system and uncertainty visualizations. Experiment 2 tested Hypotheses 3 through 9. 
A one-way analysis of variance (ANOVA) tested for significant differences in performance (superior and external rotation error, size of impingement, and time of screw placement) across all conditions (NUO, ECO, MLO, NUM, and MLM). The test found no significant differences in superior rotation error $(F=0.437, p=0.782)$, external rotation error $(F=0.927, p=0.448)$, and size of impingement $(F=0.383$, $p=0.821)$ over all conditions. The test did find significant effects of time on screw placement $(F=4.140, p<0.01)$ over all conditions.

A Tukey post hoc test makes all pair-wise comparisons of conditions in a set of data. If a significant F-value is obtained in an ANOVA, then a Tukey post hoc test indicates differences between groups for a specific condition [95]. A Tukey post hoc test was performed on all conditions for all measurements. Hypotheses 3 through 8 were analyzed using this Tukey post hoc test.

Hypothesis 3 Superior and external rotation error, size of impingement, and time of screw placement is better with multi-slice navigation (NUM) than with orthographic view navigation (NUO) under the no uncertainty visualization method.

The Tukey post hoc test showed a significant effect of navigation method (under no uncertainty visualization) on time $(p<0.01)$. The test did not show significant effects with respect to superior rotation error $(p=0.976)$, external rotation error $(p=0.997)$, and impingement $(p=0.933)$. These results suggest that subjects inserted screws faster with the multi-slice navigation method (mean $156 \mathrm{~s} \pm 6 \mathrm{SE}$ ) than with the orthographic navigation (mean $192 \mathrm{~s} \pm 10 \mathrm{~s} \mathrm{SE}$ ).

These results do not imply anything with respect to superior and external rotation error and impingement, so instead, equivalence testing was employed. The test showed 
Table 5.4: Reported Means For All Measurements According Visualization Type

\begin{tabular}{|c|c|cccc|}
\hline Type & View & $\begin{array}{c}\text { Sup. Rot. } \\
\text { Err. }\left(^{\circ}\right.\end{array}$ & $\begin{array}{c}\text { Ext. Rot. } \\
\text { Err. }\left(^{\circ}\right)\end{array}$ & $\begin{array}{c}\text { Breach } \\
(\mathrm{mm})\end{array}$ & $\begin{array}{c}\text { Time } \\
(\mathrm{s})\end{array}$ \\
& & $\begin{array}{c}\mathrm{m}) \\
\text { mean } \pm \pm \mathrm{SE}\end{array}$ & mean $\pm \pm \mathrm{SE}$ & mean $\pm \pm \mathrm{SE}$ & mean $\pm \pm \mathrm{SE}$ \\
\hline none & ortho view & $1.5 \pm 0.6$ & $-2.1 \pm 0.6$ & $0.1 \pm 0.0$ & $192 \pm 10$ \\
ellipse & ortho view & $1.3 \pm 0.6$ & $-2.0 \pm 0.6$ & $0.1 \pm 0.1$ & $156 \pm 6$ \\
lines & ortho view & $2.1 \pm 0.7$ & $0.9 \pm 0.6$ & $0.2 \pm 0.1$ & $172 \pm 8$ \\
none & multi view & $2.0 \pm 0.5$ & $-1.8 \pm 0.6$ & $0.2 \pm 0.1$ & $156 \pm 6$ \\
lines & multi view & $1.3 \pm 0.6$ & $-2.3 \pm 0.6$ & $0.2 \pm 0.1$ & $177 \pm 8$ \\
\cline { 2 - 6 } & TOTAL & $1.6 \pm 0.6$ & $-1.8 \pm 0.6$ & $0.1 \pm 0.0$ & $171 \pm 3$ \\
\hline
\end{tabular}

that the theoretical difference is within a clinically unimportant interval for superior rotation error $\left(\Delta=2^{\circ}, p<0.05\right)$, external rotation error $\left(\Delta=2^{\circ}, p<0.01\right)$, and impingement $(\Delta=2 \mathrm{~mm}, p<0.01)$.

Mean superior rotation error, external rotation error, impingement, and time for the multi-slice navigation with no uncertainty were $2.0^{\circ} \pm 0.5^{\circ} \mathrm{SE},-1.8^{\circ} \pm 0.4^{\circ} \mathrm{SE}$, $0.2 \mathrm{~mm} \pm 0.1 \mathrm{~mm} \mathrm{SE}$, and $156 \mathrm{~s} \pm 6 \mathrm{~s} \mathrm{SE}$, respectively. Mean superior rotation error, external rotation error, impingement, and time for the orthographic navigation with no uncertainty were $1.5^{\circ} \pm 0.6^{\circ} \mathrm{SE},-2.1^{\circ} \pm 0.6^{\circ} \mathrm{SE}, 0.1 \mathrm{~mm} \pm 0.0 \mathrm{~mm} \mathrm{SE}$, and 192 $\mathrm{s} \pm 10 \mathrm{~s}$ SE, respectively. Table 5.4 lists the means for all measurements according to visualization type. Figure 5.17 depicts box plots for all visualization types.

Hypothesis 4 Superior and external rotation error, size of impingement, and time of screw placement is better in multi-slice navigation (MLM) than with orthographic view navigation (MLO) with the multi-lines uncertainty visualization method.

Tukey post hoc tests did not show significant effects of navigation method with multiline uncertainty visualization with respect to superior rotation error $(p=0.880)$, 

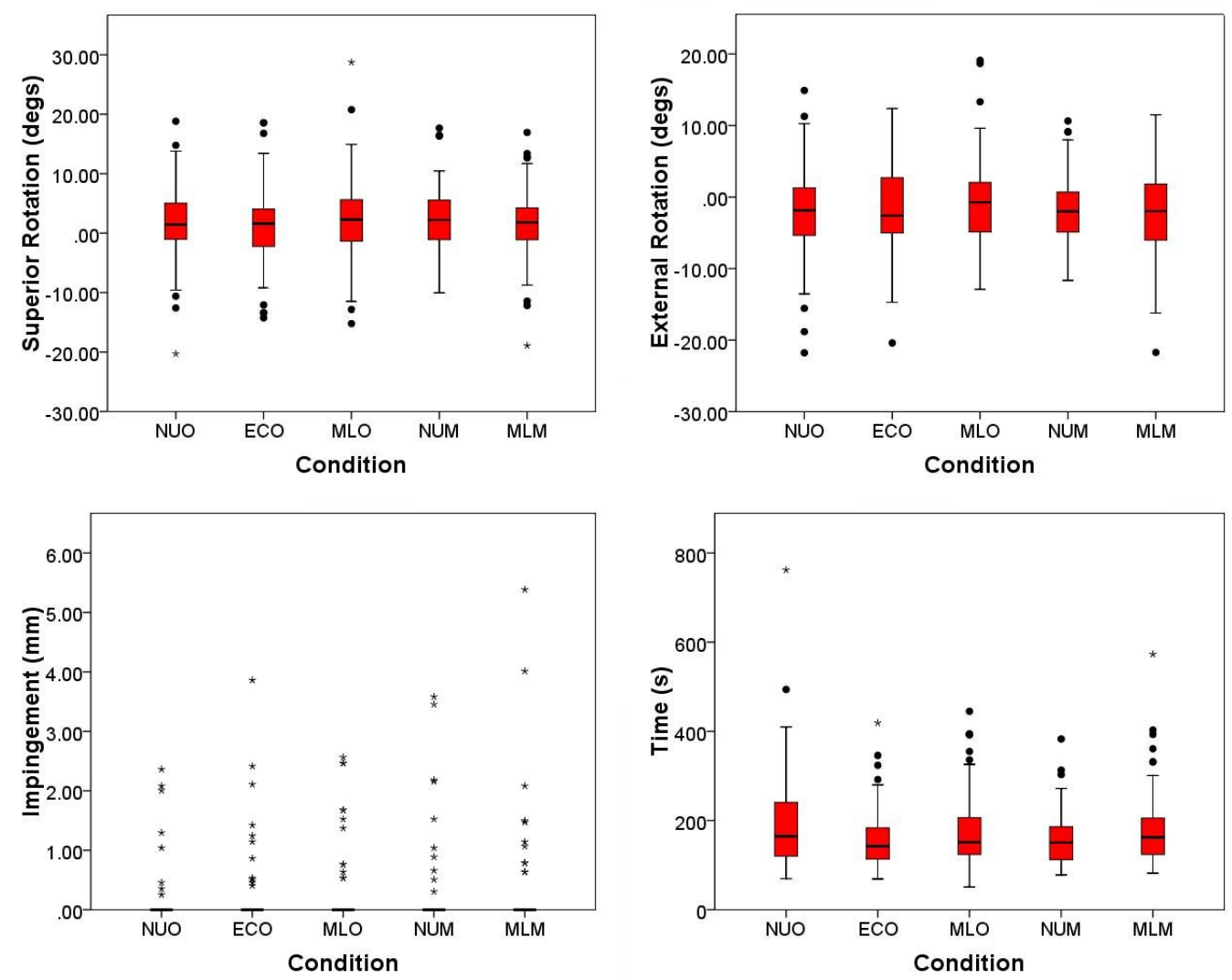

Figure 5.17: The mean of (top left) superior rotation error, (top right) external rotation error, (bottom left) impingement, and (bottom right) time plotted against visualization type for $n=104$ trials of each type.

external rotation error $(p=0.414)$, impingement $(p=0.998)$, or time $(p=0.991)$.

Equivalence tests showed that the theoretical difference between navigation methods using multi-line visualization was within a clinically unimportant interval for impingement $(\Delta=2 \mathrm{~mm}, p<0.01)$. Equivalence was not found with respect to superior rotation error $\left(\Delta=2^{\circ}, t_{L}=3.2553, p<0.01, t_{R}=1.3951, p=0.166\right)$ or external rotation error $\left(\Delta=2^{\circ}, t_{L}=4.1598, p<0.001, t_{R}=0.8220,0.413\right)^{3}$.

\footnotetext{
${ }^{3}$ Testing equivalence is a two-sided test so the left and right test statistic $t_{L}$ and $t_{R}$ and the corresponding significance should be reported. $t_{L}$ and $t_{R}$ must both exceed the critical value for equivalence [92].
} 
Mean superior rotation error, external rotation error, impingement, and time for the orthographic view navigation with multi lines were $2.1^{\circ} \pm 0.7^{\circ} \mathrm{SE},-1.0^{\circ} \pm 0.6^{\circ}$ $\mathrm{SE}, 0.2 \mathrm{~mm} \pm 0.1 \mathrm{~mm} \mathrm{SE}$, and $172 \mathrm{~s} \pm 8 \mathrm{~s}$ SE, respectively. Mean superior rotation error, external rotation error, impingement, and time with multi-view navigation and multi-lines visualization were $1.3^{\circ} \pm 0.6^{\circ} \mathrm{SE},-2.3^{\circ} \pm 0.6^{\circ} \mathrm{SE}, 0.2 \mathrm{~mm} \pm 0.1 \mathrm{~mm}$ $\mathrm{SE}$, and $177 \mathrm{~s} \pm 8 \mathrm{~s} \mathrm{SE}$, respectively. Table 5.4 lists the means for all measurements according to visualization type.

Hypothesis 5 Superior and external rotation error, size of impingement, and time of screw placement is better with ellipse uncertainty visualization (ECO) than no uncertainty visualization (NUO) under orthographic navigation.

Tukey post hoc tests showed a significant effect of uncertainty visualization (under orthographic navigation) with respect to time $(p<0.01)$. The analysis did not show significant effects with respect to superior rotation error $(p=0.999)$, external rotation error $(p=1.000)$, and impingement $(p=0.971)$.

Equivalence tests showed that the theoretical difference was within a clinically unimportant interval for superior rotation error $\left(\Delta=2^{\circ}, p<0.01\right)$, external rotation error $\left(\Delta=2^{\circ}, p<0.01\right)$, and impingement $(\Delta=2 \mathrm{~mm}, p<0.01)^{4}$.

Mean superior rotation error, external rotation error, impingement, and time for the orthographic navigation with no uncertainty were $1.5^{\circ} \pm 0.6^{\circ} \mathrm{SE},-2.1^{\circ} \pm 0.6^{\circ}$ $\mathrm{SE}, 0.1 \mathrm{~mm} \pm 0.0 \mathrm{~mm} \mathrm{SE}$, and $192 \mathrm{~s} \pm 10 \mathrm{~s}$ SE, respectively. Mean superior rotation error, external rotation error, impingement, and time for the orthographic navigation with ellipse uncertainty were $1.3^{\circ} \pm 0.6^{\circ} \mathrm{SE},-2.0^{\circ} \pm 0.6^{\circ} \mathrm{SE}, 0.1 \mathrm{~mm} \pm 0.1 \mathrm{~mm}$

\footnotetext{
${ }^{4}$ Equivalence tests were not performed with respect to time, because how to define a clinically important interval of time is not obvious.
} 
$\mathrm{SE}$, and $156 \mathrm{~s} \pm 6 \mathrm{~s} \mathrm{SE}$, respectively. Table 5.4 lists the means for all measurements according to visualization type.

Hypothesis 6 Superior and external rotation error, size of impingement, and time of screw placement is better with the multi-line uncertainty (MLO) than no uncertainty visualization (NUO) under orthographic navigation.

Tukey post hoc tests did not support the hypothesis with respect to superior rotation error $(p=0.947)$, external rotation error $(p=0.570)$, impingement $(p=$ $0.907)$, or time $(p=0.333)$.

Equivalence tests showed that the theoretical difference between multi-line uncertainty and no uncertainty visualization was within a clinically unimportant interval for impingement $(\Delta=2 \mathrm{~mm}, p<0.01)$. Equivalence could not be inferred with respect to superior rotation error $\left(\Delta=2^{\circ}, t_{L}=1.5363, p=0.128, t_{R}=2.9168\right.$, $p<0.01)$ or external rotation error $\left(\Delta=2^{\circ}, t_{L}=1.0659, p=0.289, t_{R}=3.9503\right.$, $p<0.001)$.

Mean superior rotation error, external rotation error, impingement, and time for the orthographic navigation with no uncertainty were $1.5^{\circ} \pm 0.6^{\circ} \mathrm{SE},-2.1^{\circ} \pm 0.6^{\circ} \mathrm{SE}$, $0.1 \mathrm{~mm} \pm 0.0 \mathrm{~mm} \mathrm{SE}$, and $192 \mathrm{~s} \pm 10 \mathrm{~s} \mathrm{SE}$, respectively. Mean superior rotation error, external rotation error, impingement, and time for the orthographic view navigation with multi lines were $2.1^{\circ} \pm 0.7^{\circ} \mathrm{SE},-1.0^{\circ} \pm 0.6^{\circ} \mathrm{SE}, 0.2 \mathrm{~mm} \pm 0.1 \mathrm{~mm} \mathrm{SE}$, and $172 \mathrm{~s} \pm 8 \mathrm{~s}$ SE, respectively. Table 5.4 lists the means for all measurements according to visualization type.

Hypothesis 7 Superior and external rotation error, size of impingement, and time of screw placement is better with ellipse uncertainty visualization (ECO) than the lines uncertainty visualization (MLO). 
Tukey post hoc tests did not support the hypothesis with respect to superior rotation error $(p=0.845)$, external rotation error $(p=0.612)$, impingement $(p=$ $0.999)$, or time $(p=0.543)$.

Equivalence tests showed that the theoretical difference between ellipse uncertainty visualization and multi-line visualization was within a clinically unimportant interval for impingement $(\Delta=2 \mathrm{~mm}, p<0.01)$. Equivalence could not be inferred with respect to superior rotation error $\left(\Delta=2^{\circ}, t_{L}=1.2703, p=0.207, t_{R}=3.2264\right.$, $p<0.01)$ or external rotation error $\left(\Delta=2^{\circ}, t_{L}=1.1236, p=0.264, t_{R}=3.8154\right.$, $p<0.001)$.

Mean superior rotation error, external rotation error, impingement, and time for the orthographic navigation with ellipse uncertainty were $1.3^{\circ} \pm 0.6^{\circ} \mathrm{SE},-2.0^{\circ} \pm$ $0.6^{\circ} \mathrm{SE}, 0.1 \mathrm{~mm} \pm 0.1 \mathrm{~mm} \mathrm{SE}$, and $156 \mathrm{~s} \pm 6 \mathrm{~s} \mathrm{SE}$, respectively. Mean superior rotation error, external rotation error, impingement, and time for the orthographic view navigation with multi lines were $2.1^{\circ} \pm 0.7^{\circ} \mathrm{SE},-1.0^{\circ} \pm 0.6^{\circ} \mathrm{SE}, 0.2 \mathrm{~mm}$ $\pm 0.1 \mathrm{~mm} \mathrm{SE}$, and $172 \mathrm{~s} \pm 8 \mathrm{~s} \mathrm{SE}$, respectively. Table 5.4 lists the means for all measurements according to visualization type.

Hypothesis 8 Superior and external rotation error, size of impingement, and time of screw placement is better with the multi-line uncertainty (MLM) than no uncertainty visualization (NUM) under multi-view navigation.

Tukey post hoc tests did not show a significant effect of multi-line uncertainty visualization or no uncertainty visualization using the multi-view navigation on superior rotation error $(p=0.932)$, external rotation error $(p=0.978)$, impingement $(p=0.995)$, or time $(p=0.277)$.

Equivalence test showed that the theoretical difference was within a clinically 
unimportant interval for superior rotation error $\left(\Delta=2^{\circ}, p<0.05\right)$, external rotation error $\left(\Delta=2^{\circ}, p<0.01\right)$, and impingement $(\Delta=2 \mathrm{~mm}, p<0.01)$. It appears that there was no significant performance difference (rotation error and impingement) between the multi-line or no uncertainty visualization (using multi-view navigation).

Mean superior rotation error, external rotation error, impingement, and time for the multi-slice navigation with no uncertainty were $2.0^{\circ} \pm 0.5^{\circ} \mathrm{SE},-1.8^{\circ} \pm 0.4^{\circ}$ $\mathrm{SE}, 0.2 \mathrm{~mm} \pm 0.1 \mathrm{~mm} \mathrm{SE}$, and $156 \mathrm{~s} \pm 6 \mathrm{~s} \mathrm{SE}$, respectively. Mean superior rotation error, external rotation error, impingement, and time multi-view navigation with lines visualization were $1.3^{\circ} \pm 0.6^{\circ} \mathrm{SE},-2.3^{\circ} \pm 0.6^{\circ} \mathrm{SE}, 0.2 \mathrm{~mm} \pm 0.1 \mathrm{~mm} \mathrm{SE}$, and 177 $\mathrm{s} \pm 8 \mathrm{~s} \mathrm{SE}$, respectively. Table 5.4 lists the means for all measurements according to visualization type.

Hypothesis 9 Superior and external rotation error, size of impingement, and time of screw placement is better with more surgical experience.

A one-way ANOVA supported that external rotation error $(p<0.001)$, size of impingement $(p<0.01)$, and time $(p<0.05)$ were improved with experience. An effect of experience on superior rotation error $(p=0.297)$ was not found. Tukey post hoc tests indicated that external rotation measurements for surgical staff were significantly better than junior $(p<0.01)$ and senior residents $(p<0.001)$. Surgical staff were significantly better than the junior residents $(p<0.001)$ at avoiding impingement. Surgical staff were also significantly faster than the senior residents $(p<0.05)$.

Table 5.5 reports the mean and standard error measures for superior rotation error, external rotation error, impingement, and time according to experience level. 
Table 5.5: Reported Means For All Measurements According to Experience Level and Visualization Type

\begin{tabular}{|c|c|c|c|c|c|c|}
\hline Status & Type & View & $\begin{array}{l}\text { Sup. Rot. } \\
\text { Err. }\left(^{\circ}\right) \\
\text { mean } \pm \text { SE }\end{array}$ & $\begin{array}{c}\text { Ext. Rot. } \\
\text { Err. }\left(^{\circ}\right) \\
\text { mean } \pm \mathrm{SE}\end{array}$ & $\begin{array}{c}\text { Breach } \\
(\mathrm{mm}) \\
\text { mean } \pm \mathrm{SE}\end{array}$ & $\begin{array}{c}\text { Time } \\
(\mathrm{s}) \\
\text { mean } \pm \mathrm{SE}\end{array}$ \\
\hline \multirow{6}{*}{$\begin{array}{l}\text { junior } \\
\text { resident }\end{array}$} & none & ortho view & $1.4 \pm 0.8$ & $-2.3 \pm 1.2$ & $0.2 \pm 0.1$ & $184 \pm 12$ \\
\hline & ellipse & ortho view & $1.4 \pm 0.7$ & $-1.1 \pm 0.7$ & $0.3 \pm 0.1$ & $155 \pm 9$ \\
\hline & lines & ortho view & $1.5 \pm 0.7$ & $0.0 \pm 1.0$ & $0.2 \pm 0.1$ & $193 \pm 15$ \\
\hline & none & multi view & $1.0 \pm 0.7$ & $-2.0 \pm 0.7$ & $0.3 \pm 0.1$ & $174 \pm 11$ \\
\hline & lines & multi view & $0.9 \pm 0.8$ & $-2.4 \pm 0.8$ & $0.3 \pm 0.1$ & $164 \pm 11$ \\
\hline & TOTAL & & $1.2 \pm 0.3$ & $-1.6 \pm 0.4$ & $0.3 \pm 0.0$ & $174 \pm 5$ \\
\hline \multirow{6}{*}{$\begin{array}{l}\text { senior } \\
\text { resident }\end{array}$} & none & ortho view & $1.0 \pm 1.5$ & $-0.9 \pm 0.8$ & $0.0 \pm 0.0$ & $241 \pm 22$ \\
\hline & ellipse & ortho view & $1.3 \pm 1.3$ & $-0.5 \pm 1.1$ & $0.1 \pm 0.1$ & $171 \pm 12$ \\
\hline & lines & ortho view & $1.5 \pm 1.4$ & $-0.5 \pm 1.0$ & $0.2 \pm 0.1$ & $168 \pm 8$ \\
\hline & none & multi view & $2.4 \pm 0.9$ & $-1.0 \pm 0.7$ & $0.1 \pm 0.0$ & $143 \pm 5$ \\
\hline & lines & multi view & $1.3 \pm 1.1$ & $-0.1 \pm 1.2$ & $0.2 \pm 0.2$ & $174 \pm 15$ \\
\hline & TOTAL & & $1.5 \pm 0.6$ & $-0.6 \pm 0.4$ & $0.1 \pm 0.0$ & $179 \pm 7$ \\
\hline \multirow{6}{*}{$\begin{array}{c}\text { surgical } \\
\text { staff }\end{array}$} & none & ortho view & $2.1 \pm 1.0$ & $-2.9 \pm 0.7$ & $0.0 \pm 0.0$ & $154 \pm 12$ \\
\hline & ellipse & ortho view & $1.0 \pm 1.3$ & $-4.6 \pm 1.1$ & $0.0 \pm 0.0$ & $144 \pm 9$ \\
\hline & lines & ortho view & $3.5 \pm 1.3$ & $-2.5 \pm 1.0$ & $0.0 \pm 0.0$ & $151 \pm 13$ \\
\hline & none & multi view & $2.8 \pm 1.2$ & $-2.4 \pm 0.9$ & $0.1 \pm 0.1$ & $148 \pm 11$ \\
\hline & lines & multi view & $1.8 \pm 1.0$ & $-4.2 \pm 1.0$ & $0.0 \pm 0.0$ & $197 \pm 15$ \\
\hline & TOTAL & & $2.2 \pm 0.5$ & $-3.3 \pm 0.4$ & $0.0 \pm 0.0$ & $159 \pm 6$ \\
\hline \multirow[t]{6}{*}{ TOTAL } & none & ortho view & $1.5 \pm 0.6$ & $-2.1 \pm 0.6$ & $0.1 \pm 0.0$ & $192 \pm 10$ \\
\hline & ellipse & ortho view & $1.3 \pm 0.6$ & $-2.0 \pm 0.6$ & $0.1 \pm 0.1$ & $156 \pm 6$ \\
\hline & lines & ortho view & $2.1 \pm 0.7$ & $-1.0 \pm 0.6$ & $0.2 \pm 0.1$ & $172 \pm 8$ \\
\hline & none & multi view & $2.0 \pm 0.5$ & $-1.8 \pm 0.4$ & $0.2 \pm 0.1$ & $156 \pm 6$ \\
\hline & lines & multi view & $1.3 \pm 0.6$ & $-2.3 \pm 0.6$ & $0.2 \pm 0.1$ & $177 \pm 8$ \\
\hline & TOTAL & & $1.6 \pm 0.3$ & $-1.8 \pm 0.2$ & $0.1 \pm 0.0$ & $171 \pm 3$ \\
\hline
\end{tabular}

Figure 5.18 shows the effect of experience on mean external rotation error, impingement, and time. It is unclear from the plot (and the statistics) whether there is a relationship between superior rotation error and experience. 

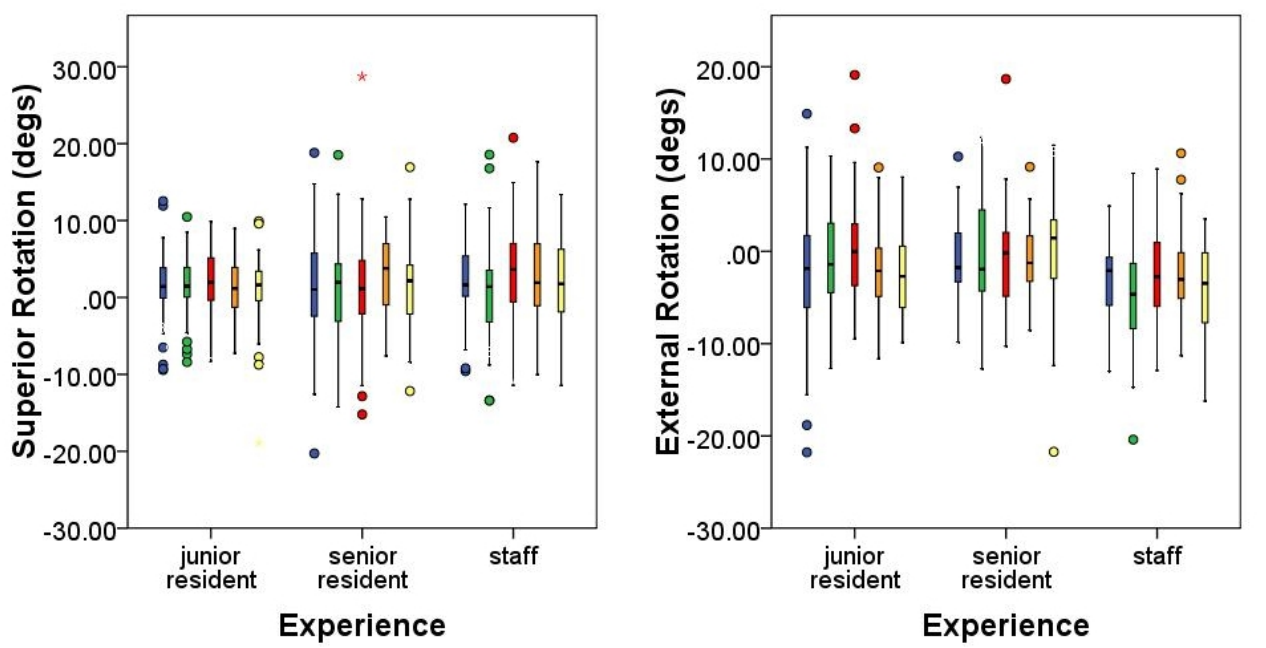

Condition

$\square$ NUO

$\square$ ECO
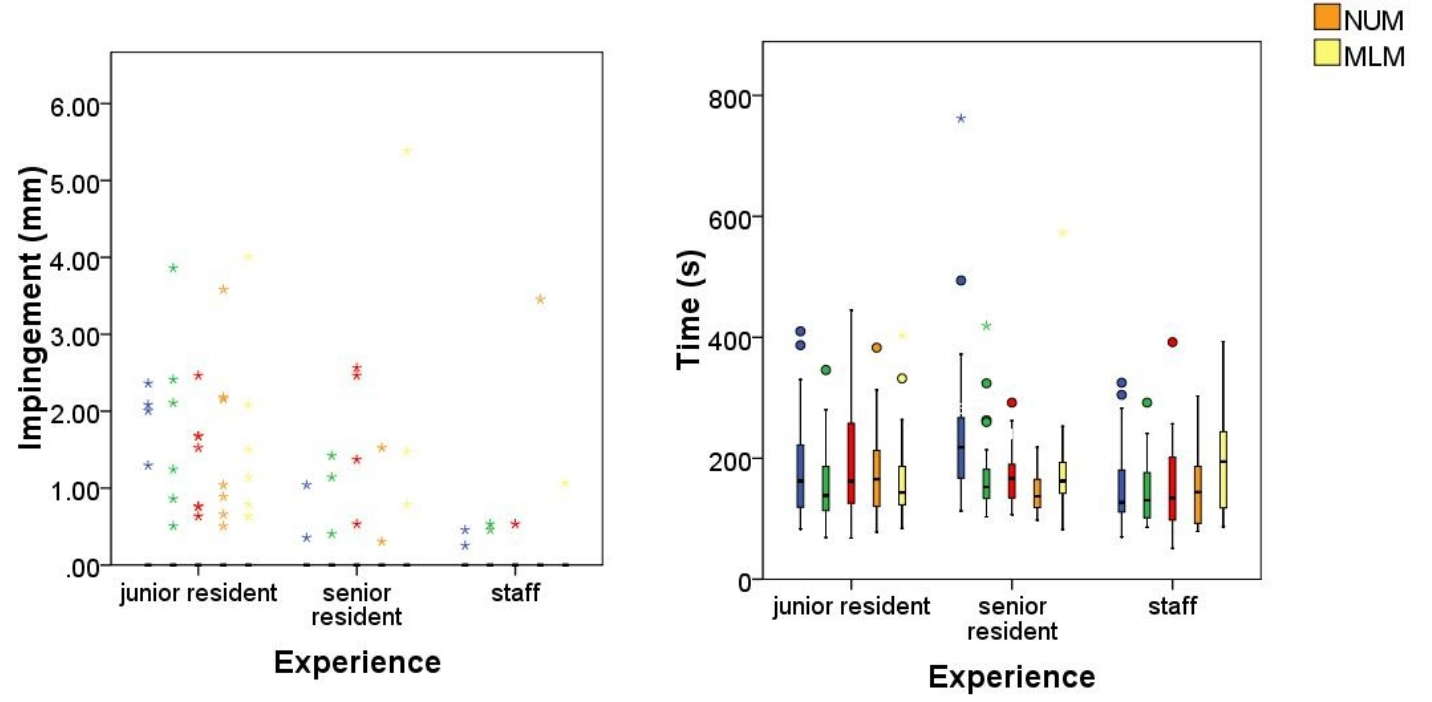

Figure 5.18: The mean of (top left) superior rotation error, (top right) external rotation error, (bottom left) impingement, and (bottom right) time plotted against surgical experience level. 


\subsubsection{Exit Questions}

Upon completion of Experiment 1 and 2, subjects were asked questions regarding their performance, visualization and navigation preferences, information regarding color-blindness, and handedness. The experimenter recorded the responses on the form detailed in Appendix F.

In the interview, subjects were asked whether they understood the ellipse or multiline uncertainty visualization better when ranking the magnitude of uncertainty in Experiment 1. Twelve of fifteen subjects stated that performance was better with multi-line uncertainty visualization. Table 5.6 summarizes interview responses.

Subjects were asked which uncertainty visualization method (ellipse and cones, multi lines, or no uncertainty) they performed best with when inserting screws in Experiment 2. Six of thirteen subjects reported that they performed best with the multi-line uncertainty, three of thirteen subjects performed best with ellipse and cone uncertainty, and four of thirteen subjects performed best when no uncertainty was shown.

Subjects were asked which visualization method (ellipse and cones, multi lines, or no uncertainty) they performed worst with when inserting screws in Experiment 2. One of thirteen subjects reported that they performed worst with the multi-line uncertainty, seven of thirteen subjects responded that they performed worst with ellipse and cone uncertainty, and two of thirteen subjects responded that they performed worst when no uncertainty was shown. Three subjects reported no adverse performance with uncertainty visualization.

Subjects were asked to explain their use of the ellipse and multi-line uncertainty visualization method. Responses are summarized in Table 5.7. 
Subjects were asked which navigation method (orthogonal views or multi-slice views) they performed best with when inserting screws in Experiment 2. Eleven of thirteen subjects stated better performance with ortho-view navigation. Two of thirteen subjects stated better performance with multi-slice navigation.

Subjects were asked to explain their guidance approach using the orthographic navigation and the multiple coronal slice navigation. Responses are summarized in Table 5.8.

Subjects were asked whether they were tired at the end of the session. Eight of thirteen subjects reported fatigue. Subjects were asked if they noticed lag in the display. Only three subjects responded in the positive and all three stated that the lag did not affect their performance. Subjects were asked if they had difficulty with the targets (affixed to the tools) moving in and out of camera visibility. All subjects answered that they did not experience this difficulty.

\subsection{Discussion}

Tables 5.9, 5.10, and 5.11 summarize the results of the study by accepted hypothesis, accepted equivalence hypothesis, and rejected hypothesis, respectively.

In Experiment 1, the results for Hypothesis 1 demonstrated that subjects perceived changes in uncertainty magnitudes significantly better with the ellipse uncertainty visualization than with the multi-lines uncertainty visualization $(p<0.05)$. Mean classification correctness was quite high with both methods $(92.6 \% \pm 1.7 \% \mathrm{SE}$ for the ellipse, $87.7 \% \pm 2.0 \% \mathrm{SE}$ for the lines). Interestingly, the exit survey results indicated that 12 of 15 subjects thought that their performance was best with the multi-lines method. Hypothesis 2 determined that surgical experience did not have 
Table 5.6: Subject Responses to Exit Questions

\begin{tabular}{|l|}
\hline Question and Response \\
\hline In Experiment 1, which visualization did you understand best? \\
Ellipse 3 \\
Lines 12 \\
\hline In Experiment 2, which did you perform best with? \\
No Uncertainty 4 \\
Ellipse 3 \\
Lines 6 \\
\hline In Experiment 2, which did you perform worst with? \\
No Uncertainty 2 \\
Ellipse 7 \\
Lines 1 \\
No opinion 3 \\
\hline In Experiment 2, which navigation method did you perform best with? \\
Ortho view 11 \\
Multi view 2 \\
\hline Were you tired at the end of the session? \\
Yes 8 \\
No 5 \\
Was there lag in the system that affected your performance? \\
Yes 0 \\
No 13 \\
\hline
\end{tabular}

a significant effect on classification correctness for either uncertainty visualization method.

Subjects were given minimal instructions on the interpretation of the visualizations used in Experiment 1. In fact, they were simply told to rank the size of uncertainty that they encountered. This parallels how surgeons would use uncertainty information in the operating room. Surgeons would need to have a natural or intuitive understanding of the visualization and should not have to rely on training by the surgical system manufacturer. 
Table 5.7: Subject Responses: How Uncertainty Methods Were Used For Guidance

\begin{tabular}{|c|l|c|}
\hline $\begin{array}{c}\text { Uncertainty } \\
\text { Visualization }\end{array}$ & Response & $\begin{array}{c}\text { Number of } \\
\text { Responses }\end{array}$ \\
\hline ellipse & $\bullet$ didn't use visualization & 9 \\
and & $\bullet$ used ellipse to center tool in coronal view & 1 \\
cone & $\bullet$ used ellipse minimally & 2 \\
& • used ellipse for initial entry & 1 \\
\hline multi lines & $\bullet$ didn't use visualization & 3 \\
& $\bullet$ used lines & 1 \\
& $\bullet$ used lines to center path of tool & 2 \\
& $\bullet$ used lines to see trajectory & 4 \\
& $\bullet$ used lines to avoid breaching pedicle & 1 \\
\hline
\end{tabular}

Table 5.8: Subject Responses: How Navigation Methods Were Used For Guidance

\begin{tabular}{|c|l|c|}
\hline $\begin{array}{c}\text { Navigation } \\
\text { Method }\end{array}$ & Response & $\begin{array}{c}\text { Number of } \\
\text { Responses }\end{array}$ \\
\hline ortho view & $\begin{array}{l}\text { - used sagittal and axial views only } \\
\text { - used coronal view to get initial position and } \\
\text { axial and sagittal views to adjust orientation } \\
\text { - used axial and sagittal views for alignment } \\
\text { and coronal to confirm alignment }\end{array}$ & 3 \\
\hline multi view & $\begin{array}{l}\bullet \text { used sagittal and axial views only } \\
\text { - used coronal view to judge depth of screw } \\
\text { - used coronal view to get position and sagittal } \\
\text { and axial views for orientation } \\
\text { - used axial and sagittal views for alignment } \\
\text { coronal to confirm alignment }\end{array}$ & 2 \\
\hline
\end{tabular}


Table 5.9: Summary of Experiment Results for Accepted Hypotheses

\begin{tabular}{|l|c|c|}
\hline Hypothesis & Sig. & Sig. p-value \\
\hline $\begin{array}{l}\text { Hypothesis 1: } \\
\text { Ranking uncertainty sizes is better with ECO than MLO }\end{array}$ & accept & $p<0.05$ \\
\hline $\begin{array}{l}\text { Hypothesis 3: NUM vs. NUO } \\
\text { NUM is better than NUO }\end{array}$ & & \\
$\quad$ Time & accept & $p<0.01$ \\
\hline $\begin{array}{l}\text { Hypothesis 5: ECO vs. NUO } \\
\text { ECO is better than NUO }\end{array}$ & accept & $p<0.01$ \\
\hline Time & & \\
\hline $\begin{array}{l}\text { Hypothesis 9: } \\
\text { Better with experience }\end{array}$ & accept & $p<0.001$ \\
$\quad$ External rotation & accept & $p<0.01$ \\
Impingement & accept & $p<0.05$ \\
\hline
\end{tabular}

Experiment 1 used only standard orthographic views for navigation, which means that some of the three-dimensional uncertainty information was lost to the two dimensional views. It would be interesting to see if subjects can perceive uncertainty magnitudes better using three-dimensional guidance systems like the multi-slice navigation approach. However, subjects indicated that they had difficulties processing all of the guidance information from all of the views on that display simultaneously; further investigation is necessary.

In Chapter 3, it was established that visualizing uncertainty improves surgical outcomes. Though the results of that particular study were interesting, they were not surprising because subjects were expected to perform better with uncertainty visualization under less than perfect registrations. If subjects are given imperfect intraoperative information then it is the effect of performing with poor data that is being studied, rather than the affect of visualization and navigation method. In fact, 
Table 5.10: Summary of Experiment Results for Hypotheses Accepted for Equivalence

\begin{tabular}{|c|c|c|}
\hline Hypothesis & Equiv. & Equiv. p-values \\
\hline \multicolumn{3}{|l|}{ Hypothesis 3: NUM vs. NUO } \\
\hline NUM is better than NUO & & \\
\hline Superior rotation error & accept & $p<0.05$ \\
\hline External rotation error & accept & $p<0.01$ \\
\hline Impingement & accept & $p<0.01$ \\
\hline \multicolumn{3}{|l|}{ Hypothesis 4: MLM vs. MLO } \\
\hline MLM is better than MLO & & \\
\hline Impingement & accept & $p<0.01$ \\
\hline \multicolumn{3}{|l|}{ Hypothesis 5: ECO vs. NUO } \\
\hline ECO is better than NUO & & \\
\hline Superior rotation & accept & $p<0.01$ \\
\hline External rotation & accept & $p<0.01$ \\
\hline Impingement & accept & $p<0.01$ \\
\hline \multicolumn{3}{|l|}{ Hypothesis 6: MLO vs. NUO } \\
\hline $\begin{array}{l}\text { MLO is better than NUO } \\
\text { Impingement }\end{array}$ & accept & $p<0.01$ \\
\hline \multicolumn{3}{|l|}{ Hypothesis 7: ECO vs. MLO } \\
\hline $\begin{array}{l}\text { ECO is better than MLO } \\
\text { Impingement }\end{array}$ & accept & $p<0.01$ \\
\hline \multicolumn{3}{|l|}{ Hypothesis 8: MLM vs. NUM } \\
\hline Superior rotation & accept & $p<0.05$ \\
\hline External rotation & accept & $p<0.01$ \\
\hline Impingement & accept & $p<0.01$ \\
\hline
\end{tabular}

it is likely to be easy to set up an experiment in which subjects always perform better with uncertainty than without.

The question investigated in Experiment 2 was: in the presence of ideal intraoperative information, how does navigation and uncertainty visualization method effect surgical performance? Surgical performance was assessed based on angular errors in screw placement, impingement of the screw into the vertebral foramen, and the time 
Table 5.11: Summary of Experiment Results for Rejected Hypotheses

\begin{tabular}{|l|c|c|}
\hline Hypothesis & Sig. p-value & Equiv. p-value \\
\hline Hypothesis 2: & & \\
Ranking better with experience \& ECO & $p=0.832$ & $\mathrm{n} / \mathrm{a}$ \\
Ranking better with experience \& MLO & $p=0.852$ & $\mathrm{n} / \mathrm{a}$ \\
\hline Hypothesis 4: MLM vs. MLO & & \\
MLM is better than MLO & $p=0.880$ & $p<0.01, p=0.166$ \\
$\quad$ Superior rotation & $p=0.414$ & $p<0.001, p=0.413$ \\
$\quad$ External rotation & $p=0.991$ & $\mathrm{n} / \mathrm{a}$ \\
$\quad$ Time & & \\
\hline Hypothesis 6: MLO vs. NUO & & \\
MLO is better than NUO & $p=0.947$ & $p=0.128, p<0.01$ \\
$\quad \begin{array}{l}\text { Superior rotation } \\
\text { External rotation }\end{array}$ & $p=0.570$ & $p=0.289, p<0.001$ \\
$\quad$ Time & $p=0.333$ & $\mathrm{n} / \mathrm{a}$ \\
\hline Hypothesis 7: ECO vs. MLO & & \\
ECO is better than MLO & $p=0.845$ & $p=0.207, p<0.01$ \\
$\quad$ Superior rotation & $p=0.612$ & $p=0.264, p<0.001$ \\
$\quad$ External rotation & $p=0.543$ & $\mathrm{n} / \mathrm{a}$ \\
$\quad$ Time & & \\
\hline Hypothesis 8: MLM vs. NUM & & \\
MLM is better than NUM & & \\
$\quad$ Time & & \\
\hline Hypothesis 9: & $p=0.277$ & $\mathrm{n} / \mathrm{a}$ \\
Better with experience & \\
$\quad$ Superior rotation & & \\
\hline
\end{tabular}

to insert a pedicle screw.

The Tukey post hoc test on all conditions and for all measurements showed significant effects of navigation and visualization type on time. In particular, subjects performed faster with multi-view navigation (NUM) than ortho-view navigation without uncertainty visualization (NUO) $(p<0.01)$. Subjects also performed faster with ellipse uncertainty (ECO) than no uncertainty with ortho-view navigation (NUO) 
$(p<0.01)$. The largest difference in mean time over all conditions is 36 seconds for the NUO (192 s $\pm 10 \mathrm{~s}$ SE) and ECO (156 s $\pm 6 \mathrm{~s}$ SE) conditions (see Table 5.4 for all means) which may or may not be a compelling clinical difference.

Hypothesis 9 tested the performance measures with respect to surgical experience. External rotation, size of impingement, and time are significantly better with experience. One would expect to find that time and impingement are factors that improve with experience. The reason for differences in external rotation can be explained by the fact that the most experienced individuals choose a negative external rotational trajectory $\left(-3.3^{\circ} \pm 0.4^{\circ} \mathrm{SE}\right)$, because that is an accepted alternate surgical approach for inserting pedicle screws, that avoids medial breaching of the pedicle. Interestingly, the senior residents category appear to follow external rotation of the path as supported the mean $\left(-0.6^{\circ} \pm 0.4^{\circ} \mathrm{SE}\right)$.

One might argue that image-guided surgery techniques lessen the learning time required in complicated surgical interventions. If that were the case, then one would expect that there would be no significant difference in performance with all levels of experience. The results suggest that this may not entirely be true; performance in the study is better with experience as Hypothesis 9 tests.

Equivalence testing demonstrated that impingement of the pedicle screw into the vertebral foramen was clinically equivalent over all navigation (Hypotheses 3 and 4) and uncertainty visualization conditions (Hypotheses 5-8). Superior and external rotation errors were equivalent for multi-view (NUM) and ortho-view (NUO) navigation methods without uncertainty visualized. Superior and external rotation errors were equivalent for ellipse uncertainty (ECO) and no uncertainty (NUO) uncertainty methods with ortho-view navigation. Superior and external rotation errors 
were equivalent for multi-line uncertainty (MLM) and no uncertainty visualization (NUM) with multi-view guidance.

In the interview, seven out of thirteen subjects reported that they only used the axial and sagittal views in multi-view navigation (which is the same as simply using the ortho-view navigation). The remaining six out of thirteen subjects responded that they used the additional coronal views in the multi-view navigation. My intention behind the multi-view navigation method was to satisfy the subjects that use both types of navigation into a single system.

In the interview, nine out of thirteen subjects responded that they did not use the ellipse and cone visualization when inserting screws. In addition, ten out of thirteen subjects reported using the multi-line visualization. The subjects responded positively to the multi-line visualization because the uncertainty is projected along the path of the tool and the effect of the uncertainty on their trajectory is readily apparent.

The results of the visualization study described in Chapter 3 demonstrated that informing the user of uncertainty improves performance. The results of the study described in this chapter indicate that the level of presentation of uncertainty is insignificant.

A major limitation of the study was that it required almost three hours to complete. As a consequence, eight of thirteen subjects reported feeling tired at the end of the session. Fatigue was only reported by residents (not surgical staff or fellows). A second factor of the experiment duration was that more subjects would have been able to complete the study if it required less time.

Finally, it is important to note that pedicle screw surgeries are very successful; 
however, only one poorly placed screw is required for success to turn to failure. The complication rates are misleading because complications range from infection to neurological impairment, the latter of which is not reflected in the complication rates. The purpose of uncertainty visualization in this case is clear: to give surgeons more resources so they can make informed decisions in the operation room and reduce the likelihood of that one poorly-placed screw. 


\section{Chapter 6}

\section{Conclusions}

The goal of this dissertation was to demonstrate that uncertainty can be reliably computed in real time in the operating room and used in an effective visualization.

\subsection{Contributions}

I have made at least five contributions in my doctoral research. My contributions and a summary of each follows.

\subsubsection{Registration Uncertainty Improves Surgical Navigation}

I performed a study which determined that using registration uncertainty information improved performance when localizing an osteoid osteoma. In the study, subjects required fewer attempts to localize the tumor when uncertainty was shown $(p<0.01)$ and subjects failed less often with uncertainty $(p<0.01)$. I was able to quantify that subjects used the uncertainty distribution in their search for the tumor $(p<0.01)$. The study concluded that visualizing registration uncertainty improves efficacy with surgical navigation. 


\subsubsection{Real-time Computation of Intraoperative Measurement Uncertainty is Possible}

I proposed UKF-based methods for computing uncertainty in calibration and tracking. Using a Kalman-filter approach to tracking and calibration was not a new idea; the novelty was in verifying that these estimates produce reliable covariances. The advantage of using Kalman filtering techniques was that covariances were generated in real time as the measurements became available rather than waiting until a sufficient number were collected for direct computation of covariances.

In the course of exploring this computation, I developed a navigation system that propagated tracking, calibration, and registration uncertainty to the tip of the surgical instrument as it moved through space.

\subsubsection{Uncertainty Was Studied in an Extensive Trial of Surgeons Strati- fied by Experience}

The effect of visualization and navigation type on surgical efficacy was explored in an extensive laboratory study using expert subjects. The study applied my work in computing and visualizing uncertainty to the real-world task of inserting screws into the spine. Fifteen subjects were stratified according to surgical experience into the categories junior resident, senior resident and fellow, and surgical staff.

The apparatus included plastic bone models designed specifically for the study that mimicked the hard cortical shell and porous interior of bone. Surgical tools from the operating room at Kingston General Hospital were affixed with rigid targets using custom mounts. 


\subsubsection{There is More Than One Way to Effectively Convey Uncertainty in Surgical Navigation}

The user study of pedicle screw insertions consisted of two experiments. The first experiment showed that two uncertainty visualization methods enabled expert subjects (surgeons and surgical residents) to determine uncertainty magnitudes with a high degree of classification correctness $(90.1 \% \pm 1.3 \% \mathrm{SE}$ for both methods combined). The second experiment studied the effect of uncertainty visualization and navigation type on performance when inserting pedicle screws. I found that the choice of navigation method and uncertainty visualization did not affect angular errors in screw placement or the size of cortical breaches.

\subsubsection{Criteria Have Been Established for Computing and Visualizing Un- certainty in Surgical Navigation}

Techniques for uncertainty visualization and computation in the operating room should consider the following criteria:

- demonstrate anisotropic uncertainty,

- speak the language that surgeons understand (confidence intervals, for example),

- consider color blindness and stereo viewing issues,

- be removable (can revert to "ordinary" view),

- not obscure anatomy or structures of interest,

- be sensitive to surgical work flow, 
- be resistant to interference (for example, excess noise),

- give surgeons enough information to decide whether to continue with surgery,

- render quickly within the guidance system, and

- allow the surgeon some flexibility in the magnitude of uncertainty shown (for example, which confidence interval).

\subsection{Future Work}

This dissertation described some contributions to understanding measurement uncertainty in surgical navigation; however, many problems remain to be investigated. A sampling of unanswered questions includes:

- Is there an efficient way to guide registration point selection with uncertainty?

- Can GPU architectures be exploited for faster, or more detailed renderings of uncertainty?

- Can a similar framework of uncertainty be applied to structures that deform, such as the brain or liver?

- During the user study, orthopaedic surgeons demonstrated enthusiasm for using head-mounted displays in surgery. How could uncertainty be added to these types of displays?

- The process of determining the boundary between neighboring structures introduces uncertainty when the boundaries are indistinguishable. Can the uncertainty in the resulting three-dimensional model be reliably computed? 


\subsection{Conclusions}

I contributed methods for computing intraoperative uncertainty in calibration and tracking. To the best of my knowledge, no other research to date combined estimates of registration, calibration, and tracking uncertainty for a complete estimate of intraoperative uncertainty. I have proposed uncertainty visualization methods for surgical guidance and developed a navigation system that computes and visualizes uncertainty in the surgeon's tool as it moves. I hope this research will motivate others to include uncertainty information into surgical navigation. 


\section{Bibliography}

[1] D. Acevedo and D. H. Laidlaw. Subjective quantification of perceptual interactions among some $2 \mathrm{~d}$ scientific visualization methods. IEEE Transactions on Visualization and Computer Graphics (Proceedings Visualization / Information Visualization 2006), 12(5), September-October 2006. In Press.

[2] K. S. Arun, T. S. Huang, and S. D. Blostein. Least-squares fitting of two 3-D point sets. IEEE Transactions on Pattern Analysis and Machine Intelligence, 9(5), 1987.

[3] M. A. Audette, F. P. Ferrie, and T. M. Peters. An algorithmic overview of surface registration techniques for medical imaging. Medical Image Analysis, 4:201-217, 2000.

[4] N. Ayache. Artificial Vision for Mobile Robots: Stereo Vision and Multisensory Perception. MIT Press, Cambridge, Massachusetts, USA, 1991.

[5] N. Ayache and O. D. Faugeras. Maintaining representations of the environment of a mobile robot. In Autonomous Mobile Robots, pages 205-220. Spring-Verlag, New York, 1990. 
[6] J. Bertin. Semiology of Graphics. The University of Wisconsin Press, Madison, Wisconsin, 1983.

[7] P. Besl and N. McKay. A method for registration of 3-D shapes. IEEE Transactions on Pattern Analysis and Machine Intelligence, 14(2):239-256, 1992.

[8] P. R. Bevington. Data Reduction and Error Analysis for the Physical Sciences. McGraw-Hill, New York, New York, USA, 1969.

[9] J.M. Bland and D.G. Altman. Statistics notes: Cronbach's alpha. British Medical Journal, 314:572, 1997.

[10] R. P. Blotchen, D. Weiskopf, and T. Ertl. Texture-based visualization of uncertainty in flow fields. In IEEE Visualization, pages 647-654, 2005.

[11] P. A. Bromiley, M. Pokric, and N. A. Thacker. Empirical evaluation of covariance estimates for mutual information coregistration. In C. Barillot, D.R. Haynor, and P. Hellier, editors, MICCAI 2004, LNCS Vol. 3216, pages 607-614. Springer-Verlag, 2004.

[12] R. Brown. Animated visual vibrations as an uncertainty visualization technique. In GRAPHITE '04: Proceedings of the 2nd international conference on Computer graphics and interactive techniques in Australasia and South East Asia, pages 84-89, 2004.

[13] D. C. Dahlin and K. K. Unni. Bone Tumors: General Aspects and Data on 8,542 Cases. Thomas, Springfield, Ill., 4 edition, 1986. 
[14] DePuy Orthopaedics Inc. Ci knee essential. http://www depuyorthopaedics . com/HealthCare/RelatedDocuments/CASSOLUTIONS/CIHIPESSENTIAL/DO_ Ci_Essential_Hip_Knee_Sales_Brochure_0612-81-505.pdf.

[15] A. J. Dietz, D. B. Kynor, E. M. Friets, J. K. Triedman, and P. E. Hammer. Effects of uncertainty in camera geometry on three-dimensional catheter reconstruction from biplane fluoroscopic images. In SPIE Medical Imaging, volume 4684 of Proceedings of SPIE, pages 237-248, 2002.

[16] S. Djurcilov, K. Kim, P. F. J. Lermusiaux, and A. Pang. Visualizing scalar volumetric data with uncertainty. Computers \& Graphics, pages 239-248, 2002.

[17] J. C. Duncan and N. Ayache. Medical image analysis: Progress over two decades and the challenges ahead. IEEE Transactions on Pattern Analysis and Machine Intelligence, 22(1):85-106, 2000.

[18] D. Eberly. Least squares fitting of data, 1998. http://www.geometrictools. com/Documentation/LeastSquaresFitting.pdf.

[19] B. Efron and R. J. Tibshirani. An Introduction to the Boostrap. Chapman \& Hall, 1993.

[20] R. E. Ellis, D. J. Fleet, J. T. Bryant, J. Rudan, and P. Fenton. A method for evaluating CT-based surgical registration. In J. Troccaz, E. Grimson, and R. Mosges, editors, Computer Vision, Virtual Reality and Robotics in Medicine and Medical Robotics and Computer-Assisted Surgery CVRMed-MRCAS, LNCS Vol. 1205, pages 141-150. Springer, 2001. 
[21] R. E. Ellis, D. Kerr, J. F. Rudan, and L. Davidson. Minimally invasive excision of deep bone tumors. In Wiro J. Niessen and Max A. Viergever, editors, MICCAI, LNCS Vol. 2208, pages 1154-1156. Springer, 2001.

[22] R. Fernando, editor. GPU Gems: Programming Techniques, Tips, and Tricks for Real-Time Graphics, chapter Volume Rendering Techniques. AddisonWesley, New York, 2004.

[23] M. Feuerstein, T. Reichl, J. Vogel, J. Traub, and N. Navab. New approaches to online estimation of electromagnetic tracking errors for laparoscopic ultrasonography. Computer Aided Surgery, 13:311-323, 2008.

[24] J. M. Fitzpatrick and M. Sonka. Handbook of Medical Imaging, volume 2. SPIE Publications, 2000.

[25] M. Fleute and S. Lavallée. Building a complete surface model from sparse data using statistical shape models: Application to computer assisted knee surgery system. In W. M. Wells III, A. C. F. Colchester, and S. L. Delp, editors, MICCAI 1998, LNCS Vol. 1496, pages 879-887, Berlin, 1998. Springer.

[26] K. T. Foley, S. K. Gupta, J. R. Justis, and M. C. Sherman. Percutaneous pedicle screw fixation of the lumbar spine. Neurosurgery Focus, 10:1-8, 2001.

[27] G. M. Foody and P. Atkinson, editors. Uncertainty in Remote Sensing and GIS. Wiley, Sussex, England, 2002.

[28] D. D. Frantz, A. D. Wiles, S. E. Leis, and S. R. Kirsch. Accuracy assessment protocols for electromagnetic tracking systems. Physics in Medicine and Biology, 48:2241-2251, 2003. 
[29] J. Freixenet, X. Munoz, D. Raba, J. Marti, and X. Cufi. Yet another survey on image segementation: region and boundary information integration. Computer Vision - ECCV 2002, LNCS Vol. 2352, pages 21-25, 2002.

[30] D. B. Gennery. Visual tracking of known three-dimensional objects. International Journal of Computer Vision, 7:243-270, 1992.

[31] G. Grigoryan and P. Rheingans. Point-based probabilistic surfaces to show surface uncertainty. IEEE Transactions on Visualization and Computer Graphics, 10:564-573, 2004.

[32] R. A. Hart, B. L. Hansen, M. Shea, F. Hsu, and G. J. Anderson. Pedicle screw placement in the thoracic spine. Spine, 30:E326-E331, 2005.

[33] W. Hoff and T. Vincent. Analysis of head pose accuracy in augmented reality. IEEE Transactions on Visualization and Computer Graphics, 6:319-334, 2000.

[34] B. K. P. Horn. Closed-form solution of absolute orientation using unit quaternions. Journal of the Optical Society of America A, 4:629-642, 1987.

[35] C. D. Jackson, D. Acevedo, D. H. Laidlaw, F. Drury, E. Vote, and D. Keefe. Designer-critiqued comparison of $2 \mathrm{~d}$ vector visualization methods: a pilot study. In SIGGRAPH '03: Proceedings of the SIGGRAPH 2003 conference

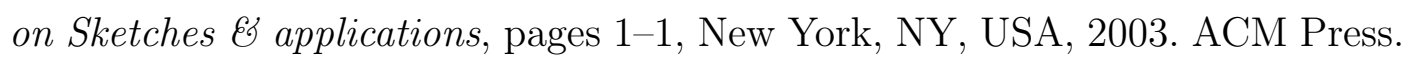

[36] B. Jaramaz, M. A. Hafez, and A. M. DiGioia III. Computer-assisted orthopaedic surgery. Proceedings of the IEEE, 94:1689-1695, 2006.

[37] C. R. Johnson and X. Tricoche. Biomedical visualization. In Pascal Verdonck, editor, Advances in Biomedical Engineering, Chapter 6. Elsevier, 2007. 
[38] D. K. Jones. Determining and visualizing uncertainty in estimates of fiber orientation from diffusion tensor MRI. Magnetic Resonance in Medicine, 49:7$12,2003$.

[39] S. Julier and J. Uhlmann. A new extension of the Kalman filter to nonlinear systems. In Proc of AeroSense, 1997.

[40] K. Kanatani. Analysis of 3-d rotation fitting. IEEE Transactions on Pattern Analysis and Machine Intelligence, 16(5):543-549, 1994.

[41] D. Kao, J. L. Dungan, and A. Pang. Visualizing 2d probability distributions from eos satellite image-derived data sets: A case study. In IEEE Visualization, pages 457-460, 2001.

[42] N. B. Karayiannia, A. Mukherjee, J. R. Glover, P. Y. Ktonas, J.D. Front, R. A. Hrachovy, and E. M. Mizrahi. Quantifying and visualizing uncertainty in EEG data of neonatal seizures. In Proceedings of the 26th Annual International Conference of the IEEE EMBS, pages 423-426, 2004.

[43] R. Khadem, C. C. Yeh, M. Sadeghi-Tehrani, M. R. Bax, J. A. Johnson, J. N. Welch, E. P. Wilkinson, and R. Shahidi. Comparative tracking error analysis of five different optical tracking systems. Computer Aided Surgery, 5:98-107, 2000.

[44] Y. J. Kim, L. G. Lenke, K. H. Bridwell, Y. S. Cho, and K. D. Riew. Free hand pedicle screw placement in the thoracic spine: Is it safe? Spine, 29:333-342, 2004 . 
[45] G. Kindlmann and J. W. Durkin. Semi-automatic generation of transfer functions for direct volume rendering. In In IEEE Symposium on Volume Visualization, pages 79-86, 1998.

[46] J. M. Kniss, R. Van Uitert, A. Stephens, G.-S. Li, T. Tasdizen, and C. Hansen. Statistically quantitative volume visualization. In IEEE Visualization, pages 287-294, 2005.

[47] E. Kraft. A quaternion-based unscented Kalman filter for orientation tracking. In Proc. IEEE 6th Int. Conf. Inf. Fusion, pages 47-54, Cairns, Queensland, Australia, 2003.

[48] M. Kunz, J. F. Rudan, G. L. Xenoyannis, and R. E. Ellis. Computer-assisted hip resurfacing using individualized drill templates. The Journal of Arthroplasty, In Press, Corrected Proof:-, 2009.

[49] M. Kunz, M. Strauss, F. Langlotz, G. Deuretzbacher, W. Rüther, and L.-P. Nolte. A non-CT based total knee arthroplasty system featuring complete softtissue balancing. In MICCAI 2001: LNCS Vol. 2208, pages 409-415. SpringerVerlag, 2001.

[50] D. H. Laidlaw, R. M. Kirby, C. D. Jackson, J. S. Davidson, T. S. Miller, M. da Silva, William H. Warren, and Michael J. Tarr. Comparing 2D vector field visualization methods: A user study. IEEE Transactions on Visualization and Computer Graphics, 11(1):59-70, 2005. 
[51] T. Laine, K. Makitalo, D. Schlenzka, K. Tallroth, M. Poussa, and A. Alho. Accuracy of pedicle screw insertion: a prospective CT study in 30 low back patients. European Spine Journal, 6:402-405, 1997.

[52] S. Lavallée. Registration for computer-integrated surgery: Methodology, state of the art. In R. H. Taylor, S. Lavallée, G. C. Burdea, and R. Mösges, editors, Computer-Integrated Surgery Technology and Clinical Applications, pages 7797, Cambridge, Massachusetts, 1996. The MIT Press.

[53] J. J. LaViola Jr. A comparison of unscented and extended Kalman filtering for estimating quaternion motion. Proceedings of the 2003 American Control Conference, 3:2435-2440, 2003.

[54] G. P. Lekovic, E. A. Potts, D. G. Karahalios, and G. Hall. A comparison of two techniques in image-guided thoracic pedicle screw placement: a retrospective study of 37 patients and 277 pedicle screws. Journal of Neurosurgery: Spine, 7:393-398, 2007.

[55] S. K. Lodha, A. Pang, R. E. Sheehan, and C. M. Wittenbrink. Uflow: Visualizing uncertainty in fluid flow. In Proceedings of the 7th conference on Visualization 1996, pages 249-254, 1996.

[56] S. K. Lodha, C. M. Wilson, and R. E. Sheehan. Listen: sounding uncertainty. In Proceedings of the 7th conference on Visualization 1996, pages 189-193., 1996.

[57] W. E. Lorensen and H. E. Cline. Marching cubes: A high resolution 3D surface construction algorithm. ACM SIGGRAPH Computer Graphics, 21(4):163-169, 1987. 
[58] B. Ma. Robust Surface-Based Registration From Sparse Measurements. Master's thesis, Department of Computing and Information Science, Queen's University, 1998.

[59] B. Ma. A Unified Approach to Surface-based Registration For Image-Guided Surgery. PhD thesis, School of Computing, Queen's University, 2005.

[60] B. Ma and R. E. Ellis. Surface-based registration with a particle filter. In C. Barillot, D. Haynor, and P. Hellier, editors, MICCAI 2007, LNCS Vol. 3216, pages 566-573. Springer, 2004.

[61] B. Ma, M. H. Moghari, R. E. Ellis, and P. Abolmaesumi. On fiducial target registration error in the presence of anisotropic noise. In N. Ayache, S. Ourselin, and A. Maeder, editors, MICCAI 2007, LNCS Vol. 4792, pages 628-635, Berlin, 2007. Springer-Verlag.

[62] B. Ma, A. L. Simpson, and R. E. Ellis. Proof of concept of a simple computerassisted technique for correcting bone deformities. In N. Ayache, S. Ourselin, and A. Maeder, editors, Medical Image Computing and Computer-Assisted Intervention, volume 4792 of Lecture Notes in Computer Science, pages 935-942. Springer, 2006.

[63] F. Maes, D. Vandermeulen, and P. Suetens. Medical image registration using mutual information. Proceedings of the IEEE, 91:1699-1722, 2003.

[64] J. B. A. Maintz and M. A. Viergever. A survey of medical image registration. Medical Image Analysis, 2:1-36, 1998. 
[65] B. Matei and P. Meer. Optimal rigid motion estimation and performance evaluation with bootstrap. In IEEE Conference on Computer Vision and Pattern Recognition, pages 339-345, 1999.

[66] C. R. Maurer, J. M. Fitzpatrick, M. Y. Wang, R. L. Galloway, R. J. Maciunas, and George S. Allen. Registration of head volume images using implantable fiducial markers. IEEE Transactions on Medical Imaging, 16:447-462, 1997.

[67] P. S. Maybeck. Stochastic Models, Estimation, and Control. Academic Press, 1979 .

[68] T. McCormick, D. Dink, S. Orcun, J. Pekny, R. Rardin, L. Baxter, V. Thai, and M. Langer. Target volume uncertainty and a method to visualize its effect on the target dose prescription. International Journal of Radiation Oncology Biology Physics, 60:1580-1588, 2004.

[69] T. McInerney and D. Terzopoulos. Deformable models in medical image analysis: A survey. Medical Image Analysis, 1:91-108, 1996.

[70] B. McQueary, L. Krause, E. Santos, H. Wang, and Q. Zhao. Modeling, analysis and visualization of uncertainty in the battlespace. In Proceedings of the 16th IEEE International Conference on Tools with Artificial Intelligence (ICTAI 2004), pages 782-783, 2004.

[71] Merriam-Webster Online. Merriam-webster online dictionary. http://www. merriam-webster.com/. 
[72] R. Minghim and A. R. Forrest. An illustrated analysis of sonification for scientific visualization. In Proceedings of the 6th Conference on Visualization 1995, pages 110-117, 1995.

[73] S. K. Mirza, G. C. Wiggins, C. Kuntz, J. E. York, C. Bellabarba, M. A. Knonodi, J. R. Chapman, and C. I. Shaffrey. Accuracy of thoracic vertebral body screw placement using standard fluoroscopy, fluoroscopic image guidance, and computed tomography image guidance. Spine, 28:402-413, 2003.

[74] M. H. Moghari, B. Ma, and P. Abolmaesumi. A theoretical comparison of different target registration error estimators. In D. Metaxas, L. Axel, G. Fichtinger, and G. Székely, editors, MICCAI 2008, LNCS Vol. 5242, pages 1032-1040, Berlin, 2008. Springer.

[75] C. Nafis, V. Jensen, L. Beauregard, and P. Anderson. Method for estimating dynamic EM racking accuracy of surgical navigation tools. In SPIE Medical Imaging, volume 6141 of Proceedings of SPIE, pages 152-167, 2006.

[76] J. G. Nagy and D. P. O’Leary. Displaying confidence images. Technical report, University of Maryland, 2000.

[77] T. S. Newman and W. Lee. On visualizing uncertainty in volumetric data: techniques and their evaluation. Journal of Visual Languages 83 Computing, 15:463-491, 2004.

[78] J. A. Noble and D. Boukerroui. Ultrasound image segmentation: a survey. IEEE Transactions on Medical Imaging, 25:987-1010, 2006. 
[79] Northern Digital Inc. Aurora technical specifications. http://www.ndigital. com/medical/aurora-techspecs-performance.php.

[80] Northern Digital Inc. NDI optical measurement technology. http://www. ndigital.com/medical/technology-optical.php.

[81] Northern Digital Inc. Optotrak certus e-type system technical specifications. http://www.ndigital.com/medical/certus-techspecs.php.

[82] N. Ohta and K. Kanatani. Optimal estimation of three-dimensional rotation and reliability evaluation. In Fifth European Conference on Computer Vision, volume 1406 of Lecture Notes in Computer Science, pages 175-187. Springer, 1998.

[83] M. Olsen, P. Gamble, M. Chiu amd N. Tumina, R. A. Boyle, and E.H. Schemitsch. The accuracy and reliability of imageless computer navigation output in hip resurfacing arthroplasty. In 9th Annual Meeting of Computer Assisted Orthopaedic Surgery International Proceedings, pages 77-79. WingSpan Press, Livermore, CA, USA, 2009.

[84] C. Olston and J. D. Mackinlay. Visualizing data with bounded uncertainty. In M. F. Costabile, editor, IEEE Symposium on Information Visualization 2002, pages $37-40,2002$.

[85] A. T. Pang, C. M. Wittenbrink, and S.K. Lodha. Approaches to uncertainty visualization. The Visual Computer, 13:370-390, 1997.

[86] X. Pennec, C. R. G. Guttmannand, and J.-P. Thirion. Feature-based registration of medical images: Estimation and validation of the pose accuracy. In 
W. M. Wells III, A. C. F. Colchester, and S. L. Delp, editors, MICCAI 1998, LNCS Vol. 1496, pages 1107-1114, Berlin, 1998. Springer.

[87] X. Pennec and J.-P. Thirion. A framework for uncertainty and validation of 3-D registration methods based on points and frames. International Journal of Computer Vision, 25:203-229, 1997.

[88] T. M. Peters and K. Cleary. Image-Guided Interventions: Technology and Applications. Springer, 2008.

[89] A. C. Rencher. Methods of Multivariate Analysis. Wiley-Interscience, New York, 1995.

[90] P. Rheingans and S. Joshi. Visualization of molecules with positional uncertainty. In Data Visualization, pages 299-306, 1999.

[91] P. J. Rhodes, R. S. Laramee, R. D. Bergeron, and T. M. Sparr. Uncertainty visualization methods in isosurface rendering. In Eurographics 2003, pages 83$88,2003$.

[92] R. H. Riffenburgh. Statistics in Medicine. Academic Press, Burlington, MA, USA, 2 edition, 2005.

[93] M. Riveiro. Evaluation of uncertainty visualization techniques for information fusion. In IEEE 2007 10th International Conference on Information Fusion, pages 1-8, 2007.

[94] R. R. Shamir. Localization and registration accuracy in image guided neurosurgery: a clinical study. International Journal of Computer Assited Radiology and Surgery, to appear, 2009. 
[95] D. J. Sheskin. Handbook of Parametric and Nonparametric Tests. Chapman \& Hall, Boca Raton, third edition, 2004.

[96] R. Sibson. Studies in the robustness of multidimensional scaling: perturbational analysis of classical scaling. Journal of the Royal Statistical Society, 41:217-229, 1979.

[97] T. Sielhorst, M. Bauer, O. Wenisch, G. Klinker, and N. Navab. Online estimation of the target registration error for n-ocular optical tracking systems. In N. Ayache, S. Ourselin, and A. Maeder, editors, MICCAI 2007, LNCS Vol. 4792, pages 652-659, Berlin, 2007. Springer-Verlag.

[98] A. L. Simpson, B. Ma, E. C. S. Chen, R. E. Ellis, and A. J. Stewart. Using registration uncertainty visualization in a user study of a simple surgical task. In M. Nielson, R. Larson, and J. Sporring, editors, Medical Image Computing and Computer-Assisted Intervention, volume 4191 of Lecture Notes in Computer Science, pages 397-404. Springer, 2006.

[99] A. L. Simpson, B. Ma, B. Slagel, D. P. Borschneck, and R. E. Ellis. Computerassisted distraction osteogenesis by Ilizarov's method. The International Journal of Medical Robotics and Computer Assisted Surgery, 4:310-320, 2008.

[100] C. V. Stewart. Covariance-based registration. Technical Report 02-08, Rensselaer Polytechnic Institute, 2002. http://www.cs.rpi.edu/research/pdf/ 02-8.pdf.

[101] D. Stewart. A platform with six degrees of freedom. Proceedings of the Institute for Mechanical Engineers, 180:371-386, 1965. 
[102] A. J. Stoddart, S. Lemke, A. Hilton, and T. Renn. Estimating pose uncertainty for surface-registration. Image and Vision Computing, 16:111-120, 1998.

[103] B. N. Taylor and C. E. Kuyatt. Guideline for evaluating and expressing the uncertainty of nist measurement results. Technical Report 1297, NIST (National Institute of Standards and Technology) Technical Note, 1994.

[104] G. P. Teitelbaum, S. Shaolian, C. G. McDougall, M. C. Preul, N. R. Crawford, and V. K. H. Sonntag. New percutaneously inserted spinal fixation system. Spine, 29:703-709, 2004.

[105] E. R. Tufte. The Visual Display of Quantitative Information. Graphics Press, 2 edition, 2001.

[106] A. R. Vaccaro and S. R. Garfin. Pedicle-screw fixation in the lumbar spine. Journal of American Academy of Orthopaedic Surgeons, 3:263-274, 1995.

[107] M. C. VanDyke, J. L. Schwartz, and C. D. Hall. Unscented kalman filtering for spacecraft attitude state and parameter estimation. In American Astronautical Society and American Institute of Aeronautics and Astronautics Space Flight Mechanics Meeting, 2004.

[108] A. Vellido and P. J. G. Lisboa. Handling outliers in brain tumor MRS data analysis through robust topographic mapping. Computers in Biology and Medicine, 36:1049-1063, 2006.

[109] R. Verma, S. Krishan, K. Haendlmayer, and A. Mohsen. Functional outcome of computer-assisted spinal pedicle screw placement: a systematic review and meta-analysis of 23 studies including 5,992 pedicle screws. European Spine 
Journal, Available Online, 2010. http://www.springerlink.com/content/ wrr88305353314w7/fulltext.pdf.

[110] N. Vlassis and A. Likas. A greedy EM algorithm for Gaussian mixture learning. Neural Processing Letters, 15:77-87, 2002.

[111] E. A. Wan and R. van der Merwe. The unscented Kalman filter for nonlinear estimation. In Proc of Sym on Adapt Sys for Sig Proc, Comm and Control, 2000.

[112] C. Ware. Information Visualization: Perception for Design. Morgan Kaufman, 2004 .

[113] E. W. Weisstein. Mathworld-A Wolfram Web Resource. http://mathworld.wolfram.com.

[114] G. Welch and G. Bishop. The Kalman filter. http://www.cs . unc. edu/ welch/ kalman/.

[115] G. Welch and G. Bishop. An introduction to the Kalman filter. Technical Report TR95-041, Department of Computer Science, University of North Carolina at Chapel Hill, 1995.

[116] G. Welch and G. Bishop. SCAAT: incremental tracking with incomplete information. In SIGGRAPH '97: Proceedings of the 24th annual conference on computer graphics and interactive techniques, pages 333-344. ACM Press, 1997.

[117] P. L. Williams and R. Warwick, editors. Gray's Anatomy. Churchill Livingstone, London, UK, 36th edition, 1980. 
[118] H. Zhang, J. E. Fritts, and S. A. Goldman. Image segmentation evaluation: A survey of unsupervised methods. Computer Vision and Image Understanding, 110:260-280, 2008.

[119] Y. J. Zhang. A survey on evaluation methods for image segmentation. Pattern Recognition, 29:1335-1346, 1996.

[120] T. Zuk. Visualizing Uncertainty. PhD thesis, Department of Computer Science, University of Calgary, 2008. 
Appendices 


\section{Appendix A}

\section{Kalman Filtering}

A comprehensive tutorial on unscented Kalman filtering has been developed by Welch and Bishop [114, 115]. The following section on state and parameter estimation is rephrased from Wan and van der Merwe [111] and Maybeck [67]. The section on unscented Kalman filtering is rephrased from the seminal paper by Julier and Uhlmann [39]. References to the large body of nonlinear estimation research can be found in the textbook by Maybeck [67].

\section{A.1 State Estimation}

A nonlinear system can be modeled using state estimation. The system is described

by the state $\mathbf{x}_{t}$ at time $t$. The basic framework for this model is typically expressed as a discrete-time nonlinear dynamic system

$$
\begin{aligned}
\mathbf{x}_{t+1} & =F\left(\mathbf{x}_{t}, \mathbf{v}_{t}\right) \\
\mathbf{y}_{t} & =H\left(\mathbf{x}_{t}, \mathbf{n}_{t}\right)
\end{aligned}
$$


where $\mathbf{y}_{t}$ is the observation of the system, $\mathbf{v}_{t}$ is the process noise, and $\mathbf{n}_{t}$ is the observation noise. The process model $F$ describes how the state changes in one time step. The observation or measurement model $H$ describes the relationship between the measured observation and the state. Both $F$ and $H$ are assumed known. Kalman filtering is a standard method for estimating the state $\mathbf{x}_{t}$. The details of this step will be discussed in Section A.4.

\section{A.2 Parameter Estimation}

Kalman filtering can be used for parameter estimation by modifying the state-space representation

$$
\begin{aligned}
\mathbf{w}_{t+1} & =\mathbf{w}_{t}+\mathbf{u}_{t} \\
\mathbf{y}_{t} & =G\left(\mathbf{x}_{t}, \mathbf{w}_{t}\right)+\mathbf{e}_{t}
\end{aligned}
$$

where $\mathbf{w}_{t}$ are the parameters to be estimated with process noise $\mathbf{u}_{t}$. A training set of known input and output $\left\{\mathbf{x}_{k}, \mathbf{d}_{k}\right\}$ with error $\mathbf{e}_{k}=\mathbf{d}_{k}-G\left(\mathbf{x}_{k}, \mathbf{w}\right)$ is provided.

\section{A.3 Dual Estimation}

Sometimes it is necessary to couple state and parameter estimation. Consider the discrete-time nonlinear dynamic system

$$
\begin{aligned}
\mathbf{x}_{t+1} & =F\left(\mathbf{x}_{t}, \mathbf{v}_{t}, \mathbf{w}\right) \\
\mathbf{y}_{t} & =H\left(\mathbf{x}_{t}, \mathbf{n}_{t}, \mathbf{w}\right)
\end{aligned}
$$


where the states $\mathbf{x}_{t}$ and the vector of model parameters $\mathbf{w}$ must be estimated simultaneously from the measurement $\mathbf{y}_{t}$.

\section{A.4 The Discrete Kalman Filter}

The Kalman filter is a standard tool for estimating linear systems. The Kalman filter uses prediction/correction in the estimation process. The process state is estimated at some time (prediction) and receives noisy measurements (correction). The equations that describe the prediction stage project forward in time the current state of the system and the error covariance to obtain the estimate for the next time step. The measurement update equations incorporate new measurements into the estimate. The estimation is computed recursively; the filter only processes the current observation rather than processing previous observations.

The extended Kalman filter (EKF) is a Kalman filter that can be used for nonlinear estimation if the process and observation models can be linearized using a Taylor series expansion. The process and observation noises are assumed to be white noise and Gaussian. The details of the EKF can be found in LaViola [53].

\section{A.5 The Unscented Kalman Filter}

The EKF is limited by the assumption that an arbitrary nonlinear function can be approximated by the linear terms of the Taylor series expansion about the mean. As a result, in highly nonlinear problems the EKF tends to be inaccurate and underestimates the true covariance of the state. This leads to poor performance and filter

divergence. Julier and Uhlmann [39] proposed the unscented Kalman filter (UKF), a nonlinear estimator to address these issues. In brief, the UKF is based on the 
idea that it is easier to approximate a Gaussian distribution than to approximate an arbitrary nonlinear function.

More specifically, a set of sigma points are chosen such that their sample mean and covariance are $\overline{\mathbf{x}}$ and $\mathbf{P}_{x}$. For each point, the nonlinear function is applied to form the transformed points with mean and covariance $\overline{\mathbf{y}}$ and $\mathbf{P}_{y}$. The $n$-dimensional random variable $\mathbf{x}$ with mean $\overline{\mathbf{x}}$ and covariance $\mathbf{P}_{x}$ is approximated by $2 n+1$ sigma points $\mathcal{X}$ (with corresponding weights $W_{i}$ ) described by

$$
\begin{aligned}
\mathcal{X}_{0} & =\overline{\mathbf{x}} \\
\mathcal{X}_{i} & =\overline{\mathbf{x}}+\left(\sqrt{(L+\lambda) \mathbf{P}_{\mathbf{x}}}\right)_{i} \quad i=1, \ldots, L \\
\mathcal{X}_{i+n} & =\overline{\mathbf{x}}-\left(\sqrt{(L+\lambda) \mathbf{P}_{\mathbf{x}}}\right)_{i-L} \quad i=L+1, \ldots, 2 L \\
W_{0}^{(m)} & =\lambda /(L+\lambda) \\
W_{0}^{(c)} & =\lambda /(L+\lambda)+\left(1-\alpha^{2}+\beta\right) \\
W_{i}^{(c)} & =W_{i}^{(c)}=1 /\{(L+\lambda)\} \quad i=1, \ldots, 2 L
\end{aligned}
$$

where $\lambda=\alpha^{2}(L+\kappa)-L$ is a scaling parameter, $\alpha$ is the spread of the scaling parameters around the mean $\overline{\mathbf{x}}, \kappa$ is a secondary scaling parameters, $\beta$ describes the distribution of $\overline{\mathbf{x}} \cdot \overline{\mathbf{x}}+\left(\sqrt{(L+\lambda) \mathbf{P}_{\mathbf{x}}}\right)_{i}$ is the $i$ th row of the matrix square root.

The unscented transform is as follows:

1. Transform each point to produce the sigma points,

$$
\mathcal{Y} \approx \mathbf{g}\left(\mathcal{X}_{i}\right) \quad i=0, \ldots, 2 L
$$


2. Compute the mean as the weighted average of the sigma points,

$$
\overline{\mathbf{y}} \approx \sum_{i=0}^{2 n} W_{i}^{(m)} \mathcal{Y}_{i}
$$

3. Compute the covariance as the weighted outer product of the sigma points,

$$
\mathbf{P}_{y} \approx \sum_{i=0}^{2 n} W_{i}^{(c)}\left\{\mathcal{Y}_{i}-\overline{\mathbf{y}}\right\}\left\{\mathcal{Y}_{i}-\overline{\mathbf{y}}\right\}^{T}
$$

The UKF equations are give in Figure A.1. UKF mean estimates have been shown to be more accurate than EKF estimates and UKF estimates of covariance have been shown to be as accurate as EKF estimates [39]. UKF implementations are much faster than EKF implementations because it is not necessary to evaluate the Jacobians required by the EKF. Unlike the EKF, the UKF is not restricted to Gaussian noise. 
Initialize with:

$$
\begin{aligned}
\hat{\mathbf{x}}_{0} & =E\left[\mathbf{x}_{0}\right] \\
\mathbf{P}_{0} & =E\left[\left(\mathbf{x}_{0}-\hat{\mathbf{x}}_{0}\right)\left(\mathbf{x}_{0}-\hat{\mathbf{x}}_{0}\right)^{T}\right] \\
\hat{\mathbf{x}}_{0}^{a}=E\left[\mathbf{x}^{a}\right] & =\left[\begin{array}{lll}
\hat{\mathbf{x}}_{0}^{T} & \mathbf{0} & \mathbf{0}
\end{array}\right]^{T} \\
\mathbf{P}_{0}^{a}=E\left[\left(\mathbf{x}_{0}^{a}-\hat{\mathbf{x}}_{0}^{a}\right)\left(\mathbf{x}_{0}^{a}-\hat{\mathbf{x}}_{0}^{a}\right)^{T}\right] & =\left[\begin{array}{ccc}
\mathbf{P}_{0} & 0 & 0 \\
0 & \mathbf{P}_{\mathbf{v}} & 0 \\
0 & 0 & \mathbf{P}_{\mathbf{n}}
\end{array}\right]
\end{aligned}
$$

For $k \in\{1, \ldots, \infty\}$,

Calculate sigma points:

$$
\mathcal{X}_{k-1}^{a}=\left[\hat{\mathbf{x}}_{k-1}^{a} \hat{\mathbf{x}}_{k-1}^{a} \pm \sqrt{(L+\lambda) \mathbf{P}_{k-1}^{a}}\right]
$$

Time update:

$$
\begin{aligned}
\mathcal{X}_{k \mid k-1}^{x} & =\mathbf{F}\left[\mathcal{X}_{k-1}^{x}, \mathcal{X}_{k-1}^{v}\right] \\
\hat{\mathbf{x}}_{\mathbf{k}}^{-} & =\sum_{i=0}^{2 L} W_{i}^{(m)} \mathcal{X}_{i, k \mid k-1}^{x} \\
\mathbf{P}_{k}^{-} & =\sum_{i=0}^{2 L} W_{i}^{(c)}\left[\mathcal{X}_{i, k \mid k-1}^{x}-\hat{\mathbf{x}}_{k}^{-}\right]\left[\mathcal{X}_{i, k \mid k-1}^{x}-\hat{\mathbf{x}}_{k}^{-}\right]^{T} \\
\mathcal{Y}_{k \mid k-1} & =\mathbf{H}\left[\mathcal{X}_{k \mid k-1}^{x}, \mathcal{X}_{k-1}^{n}\right] \\
\hat{\mathbf{y}}_{k}^{-} & =\sum_{i=0}^{2 L} W_{i}^{(m)} \mathcal{Y}_{i, k \mid k-1}
\end{aligned}
$$

Measurement update equations:

$$
\begin{aligned}
\mathbf{P}_{\tilde{\mathbf{y}}_{k}, \tilde{\mathbf{y}}_{k}} & =\sum_{i=0}^{2 L} W_{i}^{(c)}\left[\mathcal{Y}_{i, k \mid k-1}-\hat{\mathbf{y}}_{k}^{-}\right]\left[\mathcal{Y}_{i, k \mid k-1}-\hat{\mathbf{y}}_{k}^{-}\right]^{T} \\
\mathbf{P}_{\mathbf{x}_{k}, \mathbf{y}_{k}} & =\sum_{i=0}^{2 L} W_{i}^{(c)}\left[\mathcal{X}_{i, k \mid k-1}-\hat{\mathbf{x}}_{k}^{-}\right]\left[\mathcal{Y}_{i, k \mid k-1}-\hat{\mathbf{y}}_{k}^{-}\right]^{T} \\
\mathcal{K} & =\mathbf{P}_{\mathbf{x}_{k}, \mathbf{y}_{k}} \mathbf{P}_{\tilde{\mathbf{y}}_{k}, \tilde{\mathbf{y}}_{k}}^{-1} \\
\hat{\mathbf{x}}_{k} & =\hat{\mathbf{x}}_{k}^{-}+\mathcal{K}\left(\mathbf{y}_{k}-\hat{\mathbf{y}}_{k}^{-}\right) \\
\mathbf{P}_{k} & =\mathbf{P}_{k}^{-}-\mathcal{K} \mathbf{P}_{\tilde{\mathbf{y}}_{k}, \tilde{\mathbf{y}}_{k}} \mathcal{K}^{T}
\end{aligned}
$$

where, $\mathbf{x}^{a}=\left[\begin{array}{lll}\mathbf{x}^{T} & \mathbf{v}^{T} & \mathbf{n}^{T}\end{array}\right]^{T}, \mathcal{X}^{a}=\left[\begin{array}{lll}\left(\mathcal{X}^{x}\right)^{T} & \left(\mathcal{X}^{v}\right)^{T} & \left(\mathcal{X}^{n}\right)^{T}\end{array}\right]^{T}, \lambda=$ composite scaling parameter, $L=$ dimension of augmented state, $\mathbf{P}_{\mathbf{v}}=$ process noise covariance, $\mathbf{P}_{\mathbf{n}}=$ measurement noise covariance, and $W_{i}=$ weights.

Figure A.1: Unscented Kalman Filter equations. 


\section{Appendix B}

\section{Bootstrap Method}

The bootstrap method is a statistical resampling technique for computing standard error, covariance, bias, and confidence intervals by computer simulation. Efron and Tibshirani [19] provide a very thorough introduction to the bootstrap method [19]. For $n$ samples, $\hat{\theta}=\left\{z_{1}, z_{2}, \ldots, z_{n}\right\}$ of a random variable, we sample with replacement and form bootstrapped data sets. We draw a value $z_{1}^{*}$ from $\left\{z_{1}, z_{2}, \ldots, z_{n}\right\}$ and set it aside. This process is repeated $n-1$ times to form the bootstrap set $\left\{z_{1}^{*}, z_{2}^{*}, \ldots, z_{n}^{*}\right\}$

and $B$ such sets are constructed as $\left\{z_{1}^{* b}, z_{2}^{* b}, \ldots, z_{n}^{* b}\right\}$. The covariance estimate of $\hat{\theta}$ is just the covariance of $\hat{\theta}^{* b}$.

$$
\begin{gathered}
\hat{C}_{\theta}=\frac{1}{B-1} \sum_{b=1}^{B}\left[\hat{\theta}^{* b}-\bar{\theta}^{*}\right]\left[\hat{\theta}^{* b}-\bar{\theta}^{*}\right]^{T}, \\
\bar{\theta}^{*}=\frac{1}{B} \sum_{b=1}^{B} \bar{\theta}^{* b} .
\end{gathered}
$$

In practice, the bootstrap method requires 100-200 samples to obtain a good estimate of covariance. 


\section{Appendix $\mathrm{C}$}

\section{Consent Form}




\section{Evaluation of Uncertainty Visualization Methods for Computer-Assisted Surgery Letter of Information and Consent Form}

1 copy for participant. 1 signed copy for experimenter.

This research study is being conducted by Amber Simpson,(613) 533-6876, email simpson @ cs.queensu.ca and James Stewart, email jstewart ess.queensw.ca. The study is sponsored by an Natural Sciences and Engineering Research Council Collaborative Health Research Projects grant.

This study is being conducted to compare the accuracy with which a surgical probe can be placed at a target using two different visualization methods for computer guidance. One method uses a sandard computer display showing three orthogonal views of the work area for guidance. The top view corresponds to moving your surgical tool left and right. The bottom view corresponds to moving your tool up and down. The right view shows the tip of your tool as it approaches the target. You should atempe to line up your surgical tool with the target on the computer screen. If you miss the target, which you likely will on the first attempt, you should withdraw your surgical tool and attempt to find the target in another area. There is a camera tracking the position of your surgical tool so try not to block the camera while you are performing the task. You are not being timed. The second visualization is the same as the first with the exception that an ellipse representing the wncentainty in the position of the target is rendered around the target. The intuition behind the visualization is this: you will try the area of high probability (where the ellipse is whitest) first and in subsequent attempts, you will look to areas of lower probability. We expect that you would attempt to cover as much area of the distribution as possible starting in the high-probability areas.

You will be seated at a work area with a conventional computer screen one meter in frost of you that shows the visualizat ions described above. Directly in front of you is a block of modeling clay and a surgical tool. You may hold the surgical tool in whichever hand you prefer. While looking at either the computer screen or the modeling clay, you will guide the surgical tool through the modeling clay to a target obscured by the clay using the visualization provided.

We will ask you to verbally indicate to the experimenter when you have finished the task, and when you are ready for the next one. A trial will consist of ten repet itions of this task. You will perform ten trials with one type of visualization, and ten trials with another type of visualization. Before each trial, there is a short training session for you to practice moving the tool.

The entire session should last approximately one hour.

The only information we will record about you is whether you touched the target and the number of attempts in each trial. If you choose to provide any quotes, will will record them. Your name will not attributed to the quoce in any way. The only individuals who will have access to this information are the primary researchers in this study (see above). Your confidentiality is guaranteed and your performance will not be connected to your name in any publication. The research will likely be published in academic journals and conferences which will be accessible by members of the research community. You are not obliged to answer any questions that you find objectionable or which make you feel uncomfortable.

There are no known physical, psychological, economic, or social risks associated with this sudy. Your participation in this procedure is completely voluntary and you may withdraw from this study an any time without any consequences on your academic standing at Queen's University. There is no compensation nor remuneration for participating in this study. We value your time, and thank you for considering participation.

If you would like further information about the study, or have additional questions or concerns, please feel free to contact any of the researchers in this study. Your may also contact the Head of the School of Computing at Queen's University,(613) 533-6054, or the Chair of the Queen's University General Research 
Ethics Board, Dr. Joan Stevenson, (613) 533-6000 ext. 74579, email stevensj@ post.queensu.ca.

Yes No

$\square \quad \square$ I agree to have my performance reconded anonymously.

$\square$ I grant permission to publish my quotes, should I choose to provide any.

$\square$ I have read this letter of information and consent form. Any questions have been answered to my satisfaction. I may keep a copy of this letter for my records.

Name

Date

Signature 


\section{Appendix D}

\section{A Note On Constructing Plastic Bone Models}

The following is a description of building plastic bones models. These models were used in the uncertainty visualization user study of pedicle screw insertions described in Chapter 5. I chose to make my own models due to the prohibitive cost of purchasing the number required for the study. Also, I could tailor the models for my specific purposes.

In summary, these steps were undertaken to construct the models:

1. Dry human vertebrae were obtained from the anatomy library.

2. CT scans of the vertebrae were obtained.

3. The CT scans were segmented and 3D computer models were constructed.

4. The 3D models were manipulated according to my needs.

5. A 3D printer was used to produce a plastic template.

6. A two-part mould of the plastic template was made using liquid silicone rubber.

7. Copies of the plastic template were cast from the mould using casting resin and foam. 
8. The final models were sanded to smooth jagged seams.

Obtaining Vertebra Samples I borrowed dry human vertebrae samples from the anatomy library of human bone specimens at the Department of Anatomy and Cell Biology at Queen's University for use in this study. The bones are not damaged in this process.

Obtaining CT Scans CT scan of the dry human bones were made.

Constructing 3D Models A 3D model of each vertebra was constructed with software used clinically by my affiliated research group at Kingston General Hospital.

Manipulating 3D Models I wanted to remove the need to register the physical model to the $3 \mathrm{D}$ computer model, in order to reduce the time required of subjects in the study and to ensure that each subject worked with the same registration uncertainty information. To facilitate this, I modified the 3D model by removing the vertebral body and affixing an irregular polyhedron to it. I used a 3D printer to construct a mount for the model that was an exact fit for the polyhedron. I chose an irregular polyhedron to ensure that the polyhedron would fit in only one orientation. All of the bone models used the same irregular polyhedron, so only one mount was needed in the study.

Creating a Template of the Model Once I was satisfied with the 3D model, I used the $3 \mathrm{D}$ printer to produce a plastic version of the modified bone. In theory, I could have printed multiple copies of the bones in the 3D printer; however, the prototyping material is expensive and difficult to drill into. 
Creating a Mould from the Template I produced a mould of the model using Oomoo 30 silicone rubber (Smooth-On, Pennsylvania, PA, USA). I encased the model in a plastic frame. I poured the silicone rubber until half of the model was submerged. This was a very delicate step; if it was not executed correctly, the model would become trapped in the mould and the mould must be destroyed to extract the model. Once I poured the first part of the mould, I applied a releasing agent (I used vaseline diluted with paint thinner) to the surface and then poured the second part. The releasing agent allowed me to separate the two parts once the cast was set. The mould needed to cure for 24 hours.

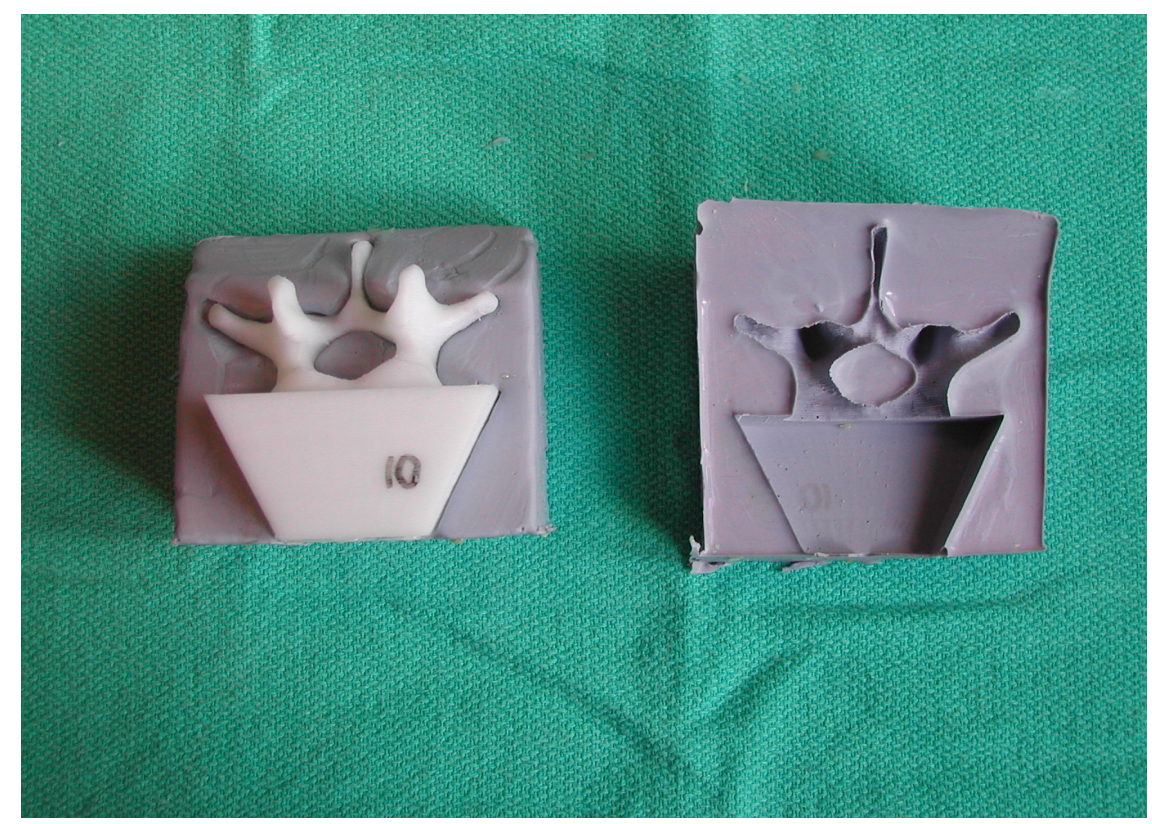

Figure D.1: The two-part mould with the template.

Casting a Copy of the Template Copies of the template were cast using Alumilite Regular Casting Resin and 610 Foam (Alumilite Corporation, Kalamazoo, MI, USA). I wanted the models to have a hard shell and porous interior, similar to bone. 
To achieve this effect, I poured a thin layer of casting resin into the mould and then filled the remainder with foam. The cast model needed about an hour before demolding.

The Final Model I removed residual material and sanded the final model to smooth the edges.

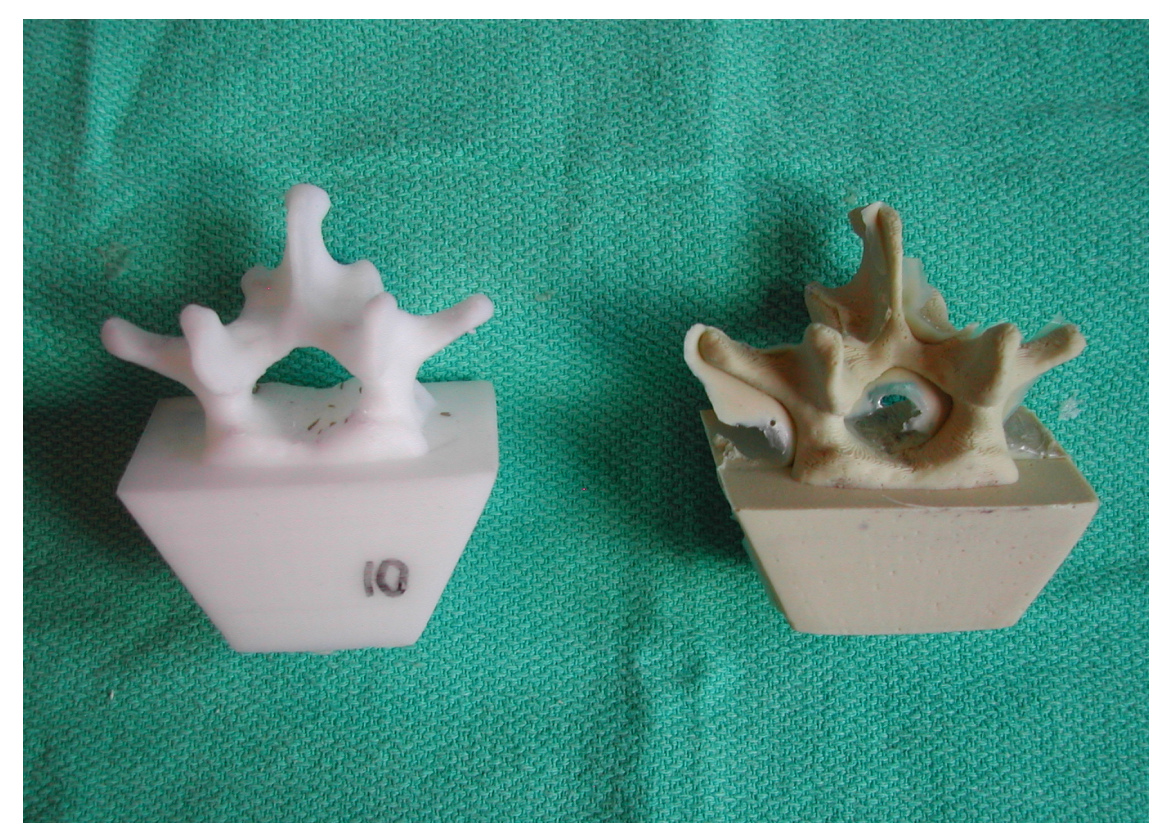

Figure D.2: The original prototyped model and the final cast model. 
Appendix E

Consent Form 


\section{Comparison of 'Techniques for Conveying Uncertainty Information in Pedicle Screw Insertion \\ Letter of Information and Consent Form}

1 copy for participant. 1 signed copy for experimenter.

This research study is being conducted by Amber Simpson, (613) 533-6876, email simpson @ cs.queensuca and James Stewart, email jstewart @ cs.queensu.ca. The study is sponsored by a Natural Sciences and Engineering Research Council Collaborative Health Research Projects grant.

This study is being conducted to compare three methods of showing error information in surgery. In the first part of the sudy, you will be shown four views of a surgical site with three different sets of uncertainty information and asked which set of uncertainty information has the largest magnitude. In the second part, you will perform simulated surgery on a plastic bone, using software and tools similar to what a surgeon would use. Directly in front of you is a plastic spine encased in foam and a screwdriver. Hold the screwdriver in whichever hand you prefer. While looking at either the computer screen or the bone model, guide the screwdriver through the plasic bone using the information displayed on the computer screen. We will use five different methods of conveying information using the computer. The methods will be randomly displayed. In all methods, there is a camera tracking the position of your surgical tool so try not to block the canera while you are performing the task. We will be timing all of your tasks; however, accuracy in your screwdriver placement is mos important. The entire session should last approximately 2.5 hours.

The only information we will record is the location of the screwdriver, once you have inserted the screw. We will ask you to complete a ques ionnaire upon finishing the viswalization tasks. If you choose to provide any comments, will will recond them. Your name will not attributed to the comment in any way. The only individuals who will have access to this information are the primary researchers in this sudy (see above). Confidentiality is guaranteed and performance will nox be connected to your name in any publication. The research will likely be published in academic journals and conferences.

There are mo known physical, psychological, economic, or social risks associated with this sudy. Your participation in this procedure is completely voluntary and you may withdraw from this study at any time without any consequences on your academic standing at Queen's University. There is no compensation or remuneration for participating in this study. We value yourtime, and than $\mathrm{k}$ you for considering participarion.

If you would like further information about the sudy, or have additional questions or concerns, please feel free to contact any of the researchers in this study. Your may also contact the Head of the School of Computing at Queen's University, (613) 533-6054, or the Chair of the Queen's University General Research Ethics Board, Dr. Joan Stevenson, (613) 533-6081, email chair.greb@queensu.ca.

Yes No

I agree to have my performance recorded anonymously.

I grant permission to publish my comments, should I choose to provide any.

I have read this letter of information and consent form. All of my questions have been answered to my satisfaction. I may keep a copy of this letter for my reconds.

Name Date

Signature 


\section{Appendix F}

\section{Questionnaire}

\section{Background}

1. Have you previously used a computer display to guide a tool in 3D?

Yes No

2. Are you ambidextrous, left or right handed?

Ambidextrous Left Right

3. To your knowledge, do you have difficulty distinguishing between colors?

Yes No If yes, which colors?

\section{Exit Questions}

1. Which visualization do you think you performed the best with? Rank each method from one to three, where one is your best performance.

No Uncertainty Ellipse Uncertainty Probe Path Uncertainty 
2. Which uncertainty display method do you think you understood better?

Ellipse Uncertainty Probe Path Uncertainty

3. Which navigation system did you perform best with?

Orthographic View Multi-slice View

4. Was it more difficult to perform the task near the end of the session?

Yes No

5. Did you notice any lag in the display?

Yes No If yes, did this affect your performance of the task? Yes No

6. Did you notice the targets moving in and out of camera visibility in the task?

Yes No

If yes, did this affect your performance of the task?

Yes No

7. How did you use each of the uncertainty visualizations?

Ellipse Uncertainty

Probe Path Uncertainty

8. How did you use each of the navigation methods?

Orthographic View

Multi-slice View

Other comments and suggestions: 